\title{
Discovery of Aminopyrazole Derivatives as Potent Inhibitors of Wild-Type and Gatekeeper Mutant FGFR2 and 3.
}

Ryan A. Brawn*, Andrew Cook, Kiyoyuki Omoto, Jiyuan Ke, Craig Karr, Federico Colombo, Milena Virrankoski, Sudeep Prajapati, Dominic Reynolds, David M. Bolduc, Tuong-Vi Nguyen, Patricia Gee, Deanna Borrelli, Benjamin Caleb, Shihua Yao, Sean Irwin, Nicholas A. Larsen, Anand Selvaraj, Xuesong Zhao, Stephanos Ioannidis.

H3 Biomedicine, 300 Technology Square, Cambridge, MA, USA

\section{Supporting Information:}

1) Synthesis

2) Intact mass spec

3) Generation of FGFR2/3 over-expressing BaF3 cell lines

4) Experimental procedure for cellular viability assays

5) Preparation of proteins for TR-FRET assays

6) FGFR TR-FRET activity assay

7) High Throughput Screen

8) Procedures for DMPK/Physiochemical assays

9) X-ray crystal structure determination and data

10) Kinase Selectivity Data

11) Institutional Animal Care and Use Committee Statement:

\section{Synthesis:}


General: Unless otherwise mentioned, all building blocks, solvents and reagents are purchased from commercial vendors and used as-is. In addition to $1 \mathrm{H}$ NMR and LCMS all final copounds were checked for HPLC purity before every biological/biochemical assay run, and any compound below $\mathbf{9 0} \%$ purity was re-purified or resynthesized before the assays were run.

\section{Compound 2:}
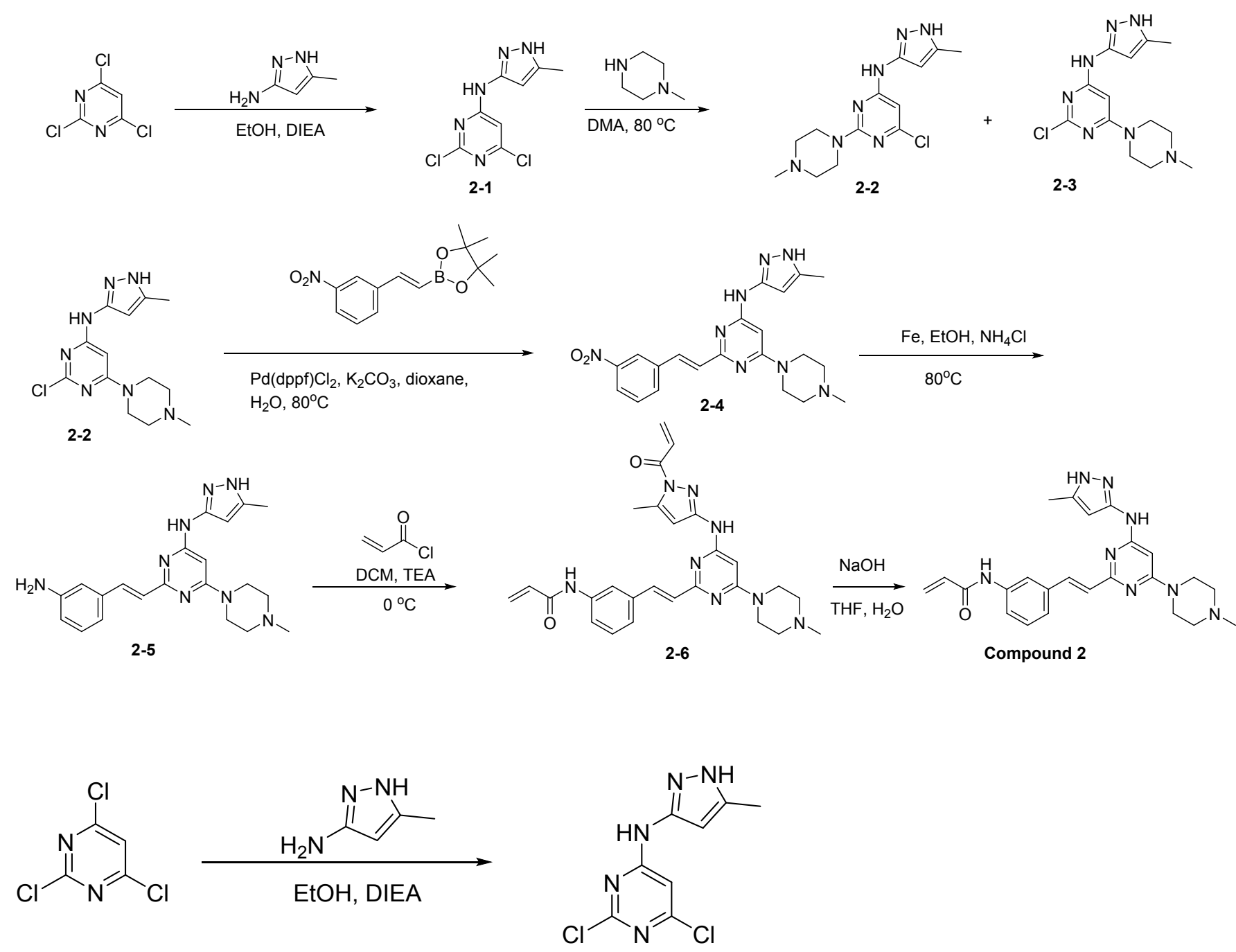

2-1

2,6-Dichloro- $\mathbf{N}$-(5-methyl-1H-pyrazol-3-yl)pyrimidin-4-amine (2-1): To a solution of 2,4,6trichloropyrimidine ( $2.44 \mathrm{~g}, 13.3 \mathrm{mmol}, 1$ equiv) in EtOH $(20 \mathrm{~mL})$ was added 5-methyl- $1 \mathrm{H}$-pyrazol-3-amine (1.29 g, $13.3 \mathrm{mmol}, 1$ equiv) and diisopropylethylamine $\left(1.72 \mathrm{~g}, 13.3 \mathrm{mmol}, 1\right.$ equiv) at $0{ }^{\circ} \mathrm{C}$. The resulting mixture was stirred at room temperature overnight. The resulting mixture was concentrated under vacuum. The crude product was diluted with ethyl acetate and washed with water. The organic phase was dried with sodium sulfate and concentrated under vacuum. This resulted in 2,6-dichloro- $\mathrm{N}$-(5-methyl-1Hpyrazol-3-yl)pyrimidin-4-amine (3.08 g, 95\%) as a light yellow solid. LCMS (ESI): [M+H]+: 244.1. 
<smiles>Cc1cc(Nc2cc(Cl)nc(Cl)n2)n[nH]1</smiles>

2-1

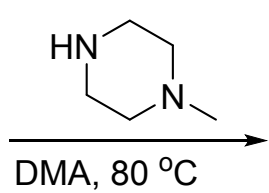

$\mathrm{Cl}$<smiles>Cc1cc(Nc2cc(N3CCN(C)CC3)nc(Cl)n2)n[nH]1</smiles>

2-2<smiles>Cc1cc(Nc2cc(Cl)nc(N3CCN(C)CC3)n2)n[nH]1</smiles>

2-3

2-Chloro-N-(5-methyl-1H-pyrazol-3-yl)-6-(4-methylpiperazin-1-yl)pyrimidin-4-amine (2-2) and 6Chloro-N-(5-methyl-1H-pyrazol-3-yl)-2-(4-methylpiperazin-1-yl)pyrimidin-4-amine (2-3): To a solution of 2,6-dichloro- $N$-(5-methyl-1H-pyrazol-3-yl)pyrimidin-4-amine $(3.1 \mathrm{~g}, 12.7 \mathrm{mmol}, 1$ equiv) in dimethylacetamide $(20 \mathrm{~mL})$ was added 1-methylpiperazine (1.27 g, $12.7 \mathrm{mmol}, 1$ equiv) and diisopropylethylamine $\left(2.46 \mathrm{~g}, 19.1 \mathrm{mmol}, 1.5\right.$ equiv). The resulting mixture was stirred at $80^{\circ} \mathrm{C}$ for 3 hours. The crude product was diluted with ethyl acetate and washed with water. The organic layer was dried with sodium sulfate and concentrated under vacuum. The crude product was purified by $\mathrm{C} 18$ reverse phase chromatography (mobile phase A: Water(0.5\% NH4HCO3), mobile phase B: ACN; gradient: $20 \%$ B to $50 \%$ B in $30 \mathrm{~min} ; 220 / 254 \mathrm{~nm}$ ) to afford 2-chloro- $N$-(5-methyl-1H-pyrazol-3-yl)-6-(4-methylpiperazin-1yl)pyrimidin-4-amine (500 mg) as a white solid and 6-chloro- $N$-(5-methyl-1H-pyrazol-3-yl)-2-(4methylpiperazin-1-yl)pyrimidin-4-amine $(2.2 \mathrm{~g}, 56 \%)$ as a white solid. LCMS (ESI): $[\mathrm{M}+\mathrm{H}]^{+}: 308.1$. Structures were assigned by $1 \mathrm{H}$ NMR analysis after de-chlorination.

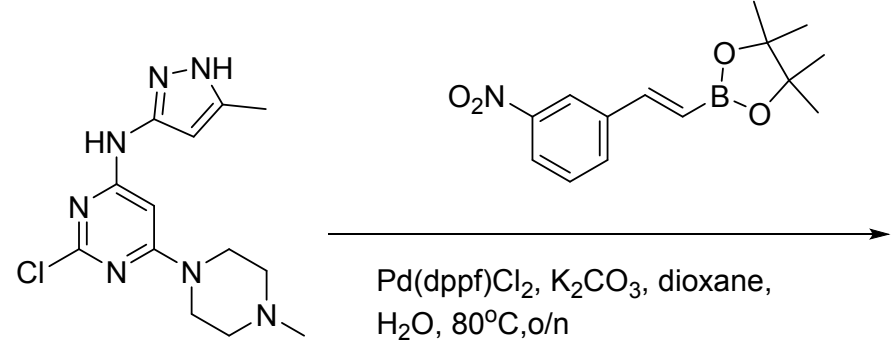

2-2

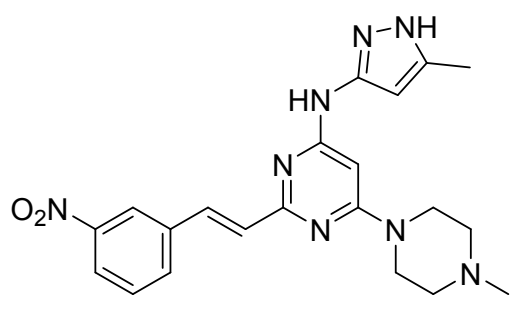

$2-4$

N-(5-Methyl-1H-pyrazol-3-yl)-6-(4-methylpiperazin-1-yl)-2-[(E)-2-(3-nitrophenyl)ethenyl]pyrimidin-4amine (2-4): To a solution of 2-chloro- $N$-(5-methyl-1H-pyrazol-3-yl)-6-(4-methylpiperazin-1-yl)pyrimidin4-amine (100 mg, $0.325 \mathrm{mmol}, 1$ equiv) and 4,4,5,5-tetramethyl-2-[(E)-2-(3-nitrophenyl)ethenyl]-1,3,2dioxaborolane (107.3 mg, $0.390 \mathrm{mmol}, 1.2$ equiv) in dioxane $(3 \mathrm{~mL})$ and $\mathrm{H}_{2} \mathrm{O}(0.3 \mathrm{~mL})$ were added $\mathrm{K}_{2} \mathrm{CO}_{3}$ (134.7 mg, $0.975 \mathrm{mmol}, 3$ equiv) and $\mathrm{Pd}(\mathrm{dppf}) \mathrm{Cl}_{2}\left(23.8 \mathrm{mg}, 0.032 \mathrm{mmol}, 0.1\right.$ equiv). After stirring at $80{ }^{\circ} \mathrm{C}$ overnight under a nitrogen atmosphere, the resulting mixture was concentrated under reduced pressure. The residue was purified by silica gel column chromatography, eluted with $\mathrm{CH}_{2} \mathrm{Cl}_{2} / \mathrm{MeOH}(10 / 1)$ to afford $\mathrm{N}$-(5-methyl-1H-pyrazol-3-yl)-6-(4-methylpiperazin-1-yl)-2-[(E)-2-(3-nitrophenyl)ethenyl]pyrimidin-4amine (100 $\mathrm{mg}, 73 \%)$ as a yellow solid. LCMS (ESI): [M+H]+: 421.2. 
<smiles>Cc1cc(Nc2cc(N3CCN(C)CC3)nc(/C=C/c3cccc([N+](=O)[O-])c3)n2)n[nH]1</smiles>

2-4

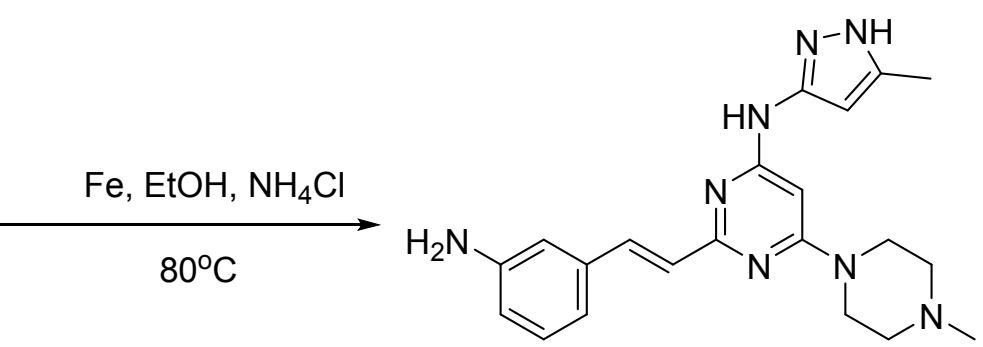

2-5

2-[(E)-2-(3-Aminophenyl)ethenyl]-N-(5-methyl-1H-pyrazol-3-yl)-6-(4-methylpiperazin-1-yl)pyrimidin-4amine (2-5): To a stirred mixture of $N$-(5-methyl-1H-pyrazol-3-yl)-6-(4-methylpiperazin-1-yl)-2-[(E)-2-(3nitrophenyl)ethenyl]pyrimidin-4-amine (50 mg, $0.119 \mathrm{mmol}, 1$ equiv) in $\mathrm{EtOH}(5 \mathrm{~mL})$ and $\mathrm{H}_{2} \mathrm{O}(1 \mathrm{~mL})$ were added iron (33.2 mg, $0.595 \mathrm{mmol}, 5$ equiv) and $\mathrm{NH}_{4} \mathrm{Cl}(63.6 \mathrm{mg}, 1.189 \mathrm{mmol}, 10$ equiv). The resulting mixture was stirred at $80^{\circ} \mathrm{C}$ for 2 hours. The solids were filtered out and the filtrate was concentrated under reduced pressure. This resulted in 2-[(E)-2-(3-aminophenyl)ethenyl]- $\mathrm{N}$-(5-methyl-1H-pyrazol-3-yl)6-(4-methylpiperazin-1-yl)pyrimidin-4-amine (60 mg, 65\%) as a brown solid. LCMS (ESI): [M+H] $]^{+} 391.2$.<smiles>Cc1cc(Nc2cc(N3CCN(C)CC3)nc(/C=C/c3cccc(N)c3)n2)n[nH]1</smiles>

$2-5$

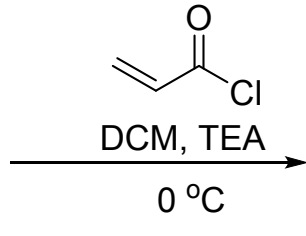<smiles>[R60]C=Cc1cccc(NC(=O)C=C)c1</smiles>

$\mathrm{N}$-[3-[(E)-2-(4-[[5-Methyl-1-(prop-2-enoyl)-1H-pyrazol-3-yl]amino]-6-(4-methylpiperazin-1-

yl)pyrimidin-2-yl)ethenyl]phenyl]prop-2-enamide (2-6): To a stirred mixture of 2-[(E)-2-(3aminophenyl)ethenyl]- $N$-(5-methyl-1H-pyrazol-3-yl)-6-(4-methylpiperazin-1-yl)pyrimidin-4-amine (46 mg, $0.118 \mathrm{mmol}, 1$ equiv) in DCM ( $3 \mathrm{~mL}$ ) was added triethylamine ( $35.8 \mathrm{mg}, 0.353 \mathrm{mmol}, 3$ equiv) and prop-2enoyl chloride $\left(21.3 \mathrm{mg}, 0.236 \mathrm{mmol}, 2\right.$ equiv) in portions at $0{ }^{\circ} \mathrm{C}$. The resulting mixture was stirred at $0{ }^{\circ} \mathrm{C}$ for 30 minutes. The crude product was concentrated under vacuum. The residue was purified by silica gel column chromatography, eluted with $\mathrm{CH}_{2} \mathrm{Cl}_{2} / \mathrm{MeOH}$ (10/1) to afford $\mathrm{N}$-[3-[(E)-2-(4-[[5-methyl-1-(prop-2enoyl)-1H-pyrazol-3-yl]amino]-6-(4-methylpiperazin-1-yl)pyrimidin-2-yl)ethenyl]phenyl]prop-2-enamide (12 mg, 20\%) as a light yellow solid. LCMS (ESI): [M+H]+: 499.2 . 


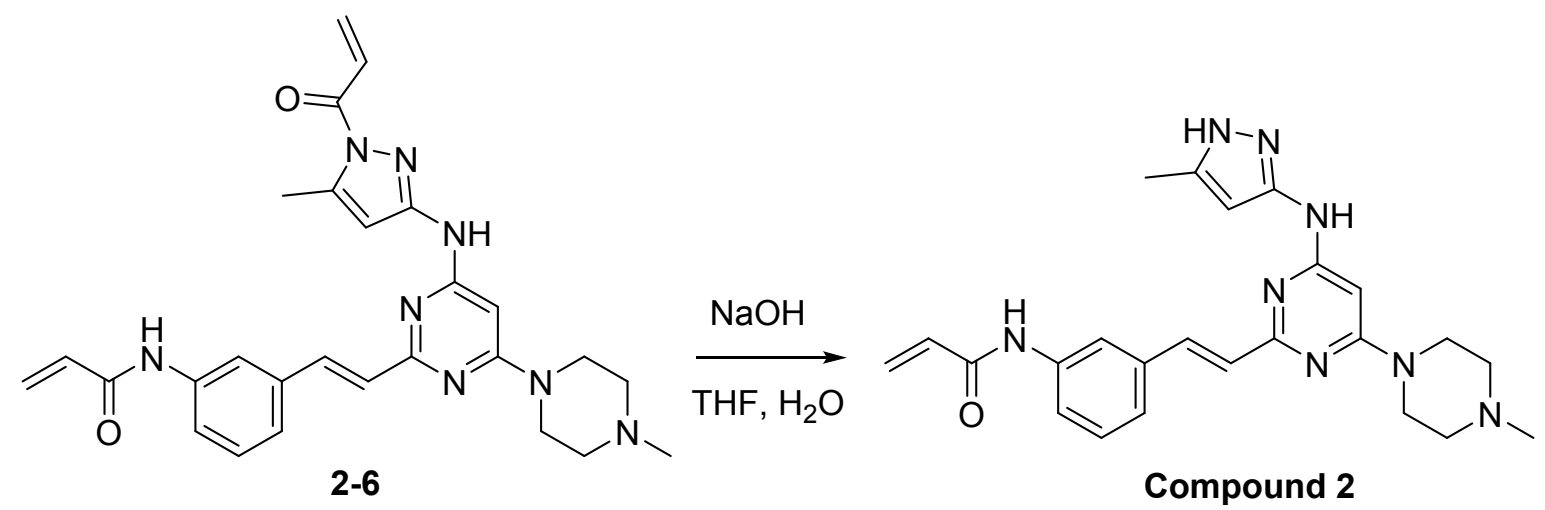

$N$-[3-[(E)-2-[4-[(5-Methyl-1H-pyrazol-3-yl)amino]-6-(4-methylpiperazin-1-yl)pyrimidin-2-

yl]ethenyl]phenyl]prop-2-enamide (Compound 2): To a stirred solution of benzyl 5-methyl-3-[[6-(4methylpiperazin-1-yl)-2-[(E)-2-[3-(prop-2-enamido)phenyl]ethenyl]pyrimidin-4-yl]amino]-1H-pyrazole-1carboxylate (70 mg, $0.121 \mathrm{mmol}, 1$ equiv) in THF $(5 \mathrm{~mL}) / \mathrm{H}_{2} \mathrm{O}(1 \mathrm{~mL})$ was added $\mathrm{NaOH}(24.2 \mathrm{mg}, 0.605$ mmol, 5 equiv). The resulting mixture was stirred at room temperature for 30 minutes. The crude product was purified by Prep-HPLC with the following conditions: Column: XBridge Shield RP18 OBD Column 30 x150 mm, 5 um; Mobile Phase A:Water (10 mmol/L NH$\left.{ }_{4} \mathrm{HCO}_{3}\right)$, Mobile Phase B: ACN; Flow rate: $60 \mathrm{~mL} / \mathrm{min}$; Gradient: $25 \%$ B to $50 \%$ B in $7 \mathrm{~min} ; 254 / 220 \mathrm{~nm}$; Rt: $6.52 \mathrm{~min}$ to afford $\mathrm{N}$-[3-[(E)-2-[4-[(5-methyl- $1 \mathrm{H}$ pyrazol-3-yl)amino]-6-(4-methylpiperazin-1-yl)pyrimidin-2-yl]ethenyl]phenyl]prop-2-enamide (33 mg, $61 \%)$ as an off-white solid. LCMS (ESI): [M+H] ${ }^{+}: 445.3 .{ }^{1} \mathrm{H}$ NMR (300 MHz, CD $\left.{ }_{3} \mathrm{OD}\right) \delta 7.93(\mathrm{~s}, 1 \mathrm{H}), 7.80(\mathrm{~d}, J$ $=15.9 \mathrm{~Hz}, 1 \mathrm{H}), 7.62-7.59(\mathrm{~m}, 1 \mathrm{H}), 7.39-7.34(\mathrm{~m}, 2 \mathrm{H}), 6.97(\mathrm{~d}, J=15.8 \mathrm{~Hz}, 1 \mathrm{H}), 6.52-6.42(\mathrm{~m}, 2 \mathrm{H}), 6.37$ $-6.36(\mathrm{~m}, 1 \mathrm{H}), 6.08(\mathrm{~s}, 1 \mathrm{H}), 5.82-5.78(\mathrm{~m}, 1 \mathrm{H}), 3.71-3.68(\mathrm{~m}, 4 \mathrm{H}), 2.57-2.54(\mathrm{~m}, 4 \mathrm{H}), 2.36(\mathrm{~s}, 3 \mathrm{H}), 2.31$ $(\mathrm{s}, 3 \mathrm{H})$.

\section{Compound 3:}




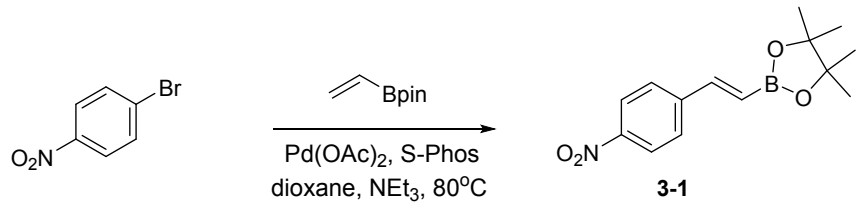

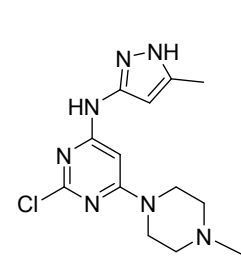

2-2

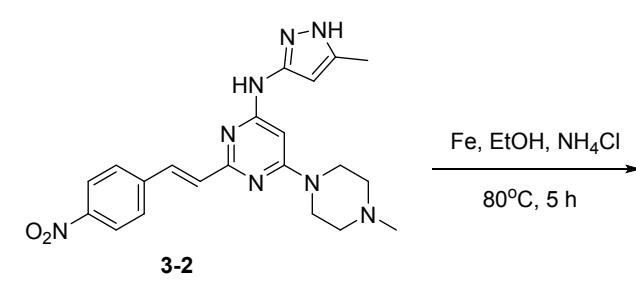

3-2
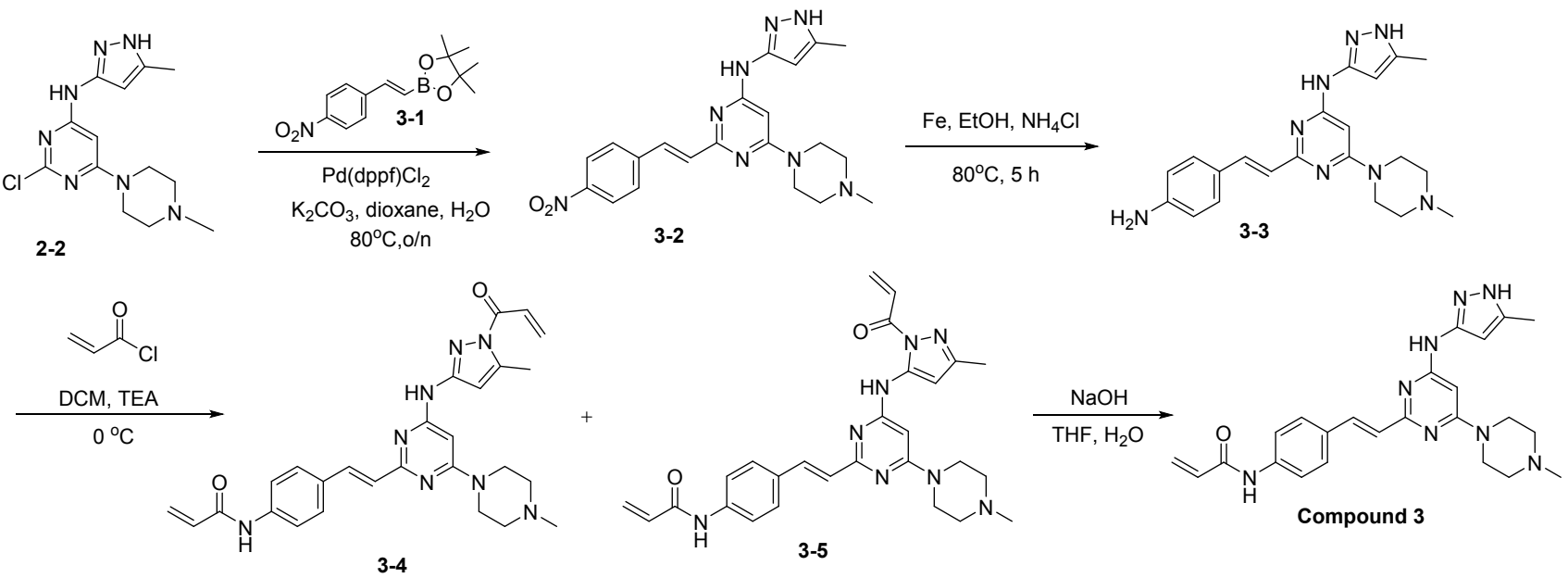<smiles>O=[N+]([O-])c1ccc(Br)cc1</smiles>

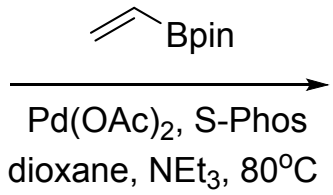<smiles>CC1(C)OB(/C=C/c2ccc([N+](=O)[O-])cc2)OC1(C)C</smiles>

\section{3-1}

4,4,5,5-Tetramethyl-2-[(E)-2-(4-nitrophenyl)ethenyl]-1,3,2-dioxaborolane (3-1): To a mixture of 1bromo-4-nitrobenzene (1.01 g, $5.0 \mathrm{mmol}, 1$ equiv) in dioxane $(15 \mathrm{~mL})$ was added 2-ethenyl-4,4,5,5tetramethyl-1,3,2-dioxaborolane (1.54 g, $10.0 \mathrm{mmol}, 2$ equiv), $\mathrm{Pd}(\mathrm{OAc})_{2}$ (44.9 mg, $0.20 \mathrm{mmol}, 0.04$ equiv), SPhos (164.2 mg, $0.40 \mathrm{mmol}, 0.08$ equiv) and $\mathrm{NEt}_{3}(2.02 \mathrm{~g}, 20 \mathrm{mmol}, 4$ equiv) at room temperature. The resulting mixture was stirred at $80^{\circ} \mathrm{C}$ overnight under a nitrogen atmosphere. The resulting mixture was diluted with ethyl acetate and washed with brine. The organic layer was dried with sodium sulfate and concentrated. The residue was purified by reverse phase chromatography to afford 4,4,5,5-tetramethyl2-[(E)-2-(4-nitrophenyl)ethenyl]-1,3,2-dioxaborolane (500 mg, 36\%) as a light orange solid. 
<smiles>Cc1cc(Nc2cc(N3CCN(C)CC3)nc(Cl)n2)n[nH]1</smiles>

2-2

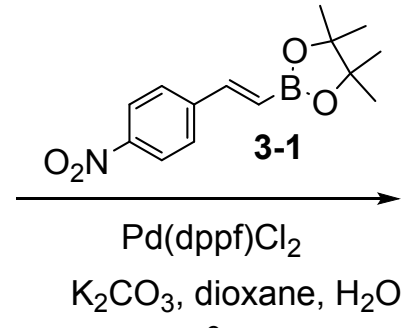

$80^{\circ} \mathrm{C}$

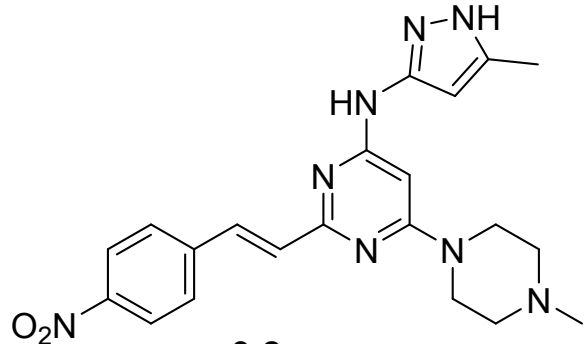

3-2

N-(5-Methyl-1H-pyrazol-3-yl)-6-(4-methylpiperazin-1-yl)-2-[(E)-2-(4-nitrophenyl)ethenyl]pyrimidin-4amine (3-2): A mixture of 2-chloro- $N$-(5-methyl-1H-pyrazol-3-yl)-6-(4-methylpiperazin-1-yl)pyrimidin-4amine (100 mg, $0.325 \mathrm{mmol}, 1$ equiv), 4,4,5,5-tetramethyl-2-[(E)-2-(4-nitrophenyl)ethenyl]-1,3,2dioxaborolane (134 mg, $0.487 \mathrm{mmol}, 1.5$ equiv), $\mathrm{Pd}(\mathrm{dppf}) \mathrm{Cl}_{2}\left(47.6 \mathrm{mg}, 0.065 \mathrm{mmol}, 0.2\right.$ equiv) and $\mathrm{K}_{2} \mathrm{CO}_{3}$ ( $89.8 \mathrm{mg}, 0.650 \mathrm{mmol}, 2$ equiv) in dioxane $(4 \mathrm{~mL})$ and $\mathrm{H}_{2} \mathrm{O}(0.4 \mathrm{~mL})$ was stirred at $80^{\circ} \mathrm{C}$ overnight under a nitrogen atmosphere. The resulting mixture was concentrated under vacuum. The residue was purified by silica gel column chromatography, eluting with $\mathrm{CH}_{2} \mathrm{Cl}_{2} / \mathrm{MeOH}(5 / 1)$ to afford $\mathrm{N}$-(5-methyl-1H-pyrazol3-yl)-6-(4-methylpiperazin-1-yl)-2-[(E)-2-(4-nitrophenyl)ethenyl]pyrimidin-4-amine (100 mg, 73\%) as a yellow solid. LCMS (ESI): [M+H]+: 421.2 .<smiles>Cc1cc(Nc2cc(N3CCN(C)CC3)nc(/C=C/c3ccc([N+](=O)[O-])cc3)n2)n[nH]1</smiles>

3-2

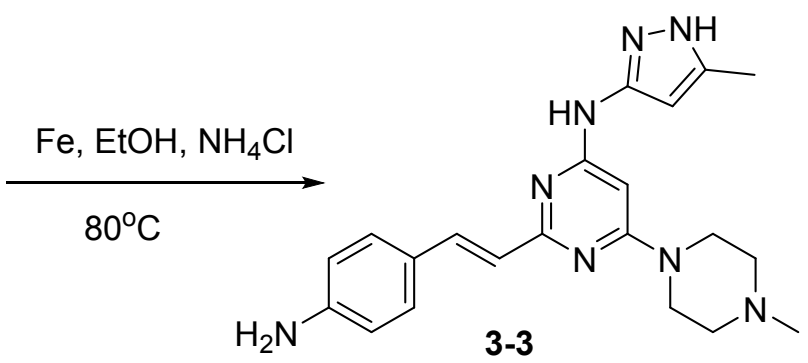

3-3

(E)-2-(4-Aminostyryl)-N-(5-methyl-1H-pyrazol-3-yl)-6-(4-methylpiperazin-1-yl)pyrimidin-4-amine (3-3): To a solution of $\quad N$-(5-methyl-1H-pyrazol-3-yl)-6-(4-methylpiperazin-1-yl)-2-[(E)-2-(4nitrophenyl)ethenyl]pyrimidin-4-amine (100 mg, $0.238 \mathrm{mmol}, 1$ equiv) in $\mathrm{EtOH}(5 \mathrm{~mL})$ and $\mathrm{H}_{2} \mathrm{O}(0.5 \mathrm{~mL})$ was added $\mathrm{NH}_{4} \mathrm{Cl}(127.2 \mathrm{mg}, 2.378 \mathrm{mmol}, 10$ equiv) and iron $(66.4 \mathrm{mg}, 1.189 \mathrm{mmol}, 5$ equiv). The resulting mixture was stirred at $80^{\circ} \mathrm{C}$ for 5 hours. The resulting mixture was filtered. The filtrate was concentrated under reduced pressure. This resulted in (E)-2-(4-aminostyryl)- $N$-(5-methyl-1H-pyrazol-3-yl)-6-(4methylpiperazin-1-yl)pyrimidin-4-amine (100 mg) as a crude solid. LCMS (ESI): [M+H]+: 391.2. 


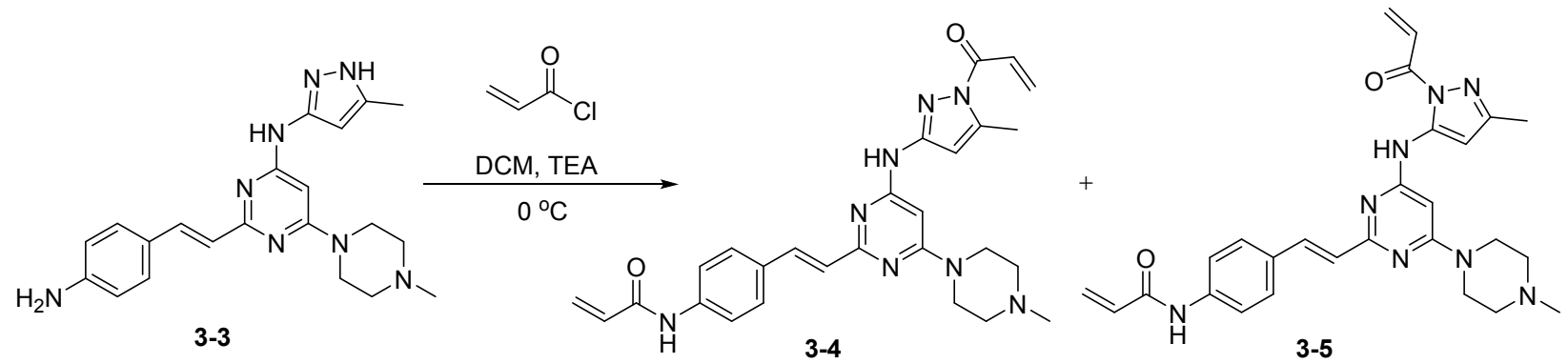

$N$-[4-[(E)-2-(4-[[5-methyl-1-(prop-2-enoyl)-1H-pyrazol-3-yl]amino]-6-(4-methylpiperazin-1yl)pyrimidin-2-yl)ethenyl]phenyl]prop-2-enamide (3-4) and (E)- $\mathrm{N}$-(4-(2-(4-(1-acryloyl-3-methyl-1Hpyrazol-5-ylamino)-6-(4-methylpiperazin-1-yl)pyrimidin-2-yl)vinyl)phenyl)acrylamide (3-5): To a stirred solution of 2-[(E)-2-(4-aminophenyl)ethenyl]- $N$-(5-methyl-1H-pyrazol-3-yl)-6-(4-methylpiperazin-1yl)pyrimidin-4-amine (94 mg, $0.241 \mathrm{mmol}, 1$ equiv) in DCM $(2.0 \mathrm{~mL}$ ) was added diisopropylethylamine (62 $\mathrm{mg}, 0.481 \mathrm{mmol}, 2$ equiv) and prop-2-enoyl chloride $\left(43.6 \mathrm{mg}, 0.481 \mathrm{mmol}, 2\right.$ equiv) dropwise at $0{ }^{\circ} \mathrm{C}$. The resulting mixture was stirred at room temperature for 1 hour. The crude product was concentrated under vacuum. The residue was purified by silica gel column chromatography, eluting with $\mathrm{CH}_{2} \mathrm{Cl}_{2} / \mathrm{MeOH}(12 / 1)$ to afford a mixture of $\mathrm{N}$-[4-[(E)-2-(4-[[5-methyl-1-(prop-2-enoyl)-1H-pyrazol-3-yl]amino]-6-(4methylpiperazin-1-yl)pyrimidin-2-yl)ethenyl]phenyl]prop-2-enamide and (E)-N-(4-(2-(4-(1-acryloyl-3methyl-1H-pyrazol-5-ylamino)-6-(4-methylpiperazin-1-yl)pyrimidin-2-yl)vinyl)phenyl)acrylamide (40 mg) as a light yellow solid. LCMS (ESI): [M+H]+: 499.2.

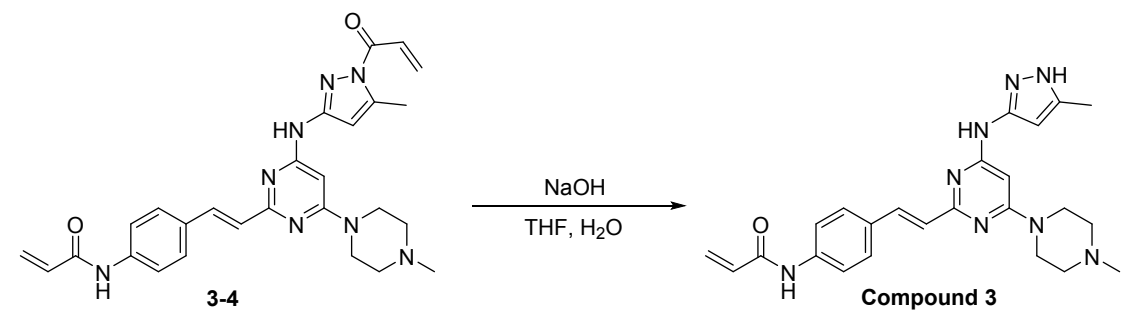

$N$-[4-[(E)-2-[4-[(5-Methyl-1H-pyrazol-3-yl)amino]-6-(4-methylpiperazin-1-yl)pyrimidin-2-

yl]ethenyl]phenyl]prop-2-enamide (Compound 3): To a stirred solution of $N$-[4-[(E)-2-(4-[[5-methyl-1(prop-2-enoyl)-1H-pyrazol-3-yl]amino]-6-(4-methylpiperazin-1-yl)pyrimidin-2-yl)ethenyl]phenyl]prop-2enamide (40 mg, $0.080 \mathrm{mmol}, 1$ equiv) in THF $(1 \mathrm{~mL})$ and $\mathrm{H}_{2} \mathrm{O}(1 \mathrm{~mL})$ was added $\mathrm{NaOH}(3.2 \mathrm{mg}, 0.080$ mmol, 1 equiv) at room temperature. The resulting mixture was stirred at room temperature for $30 \mathrm{~min}$. The crude product was purified by Prep-HPLC with following conditions: Column: XBridge Prep OBD C18 Column 30×150 mm 5um;Mobile Phase A:Water (10 mmol/L NH$\left.{ }_{4} \mathrm{HCO}_{3}\right)$, Mobile Phase B: ACN; Flow rate: $60 \mathrm{~mL} / \mathrm{min}$; Gradient: $24 \%$ B to $42 \%$ B in $7 \mathrm{~min}$; 254/220 nm; Rt: $6.25 \mathrm{~min}$ to afford N-[4-[(E)-2-[4-[(5methyl-1H-pyrazol-3-yl)amino]-6-(4-methylpiperazin-1-yl)pyrimidin-2-yl]ethenyl]phenyl]prop-2-enamide (15 mg, 42\%) as a light yellow solid. LCMS (ESI): [M+H]+: 445.4. ${ }^{1} \mathrm{H}$ NMR (300 MHz, DMSO-d $\left.d_{6}\right) \delta 11.87$ (s, $1 \mathrm{H}), 10.30(\mathrm{~s}, 1 \mathrm{H}), 9.19(\mathrm{~s}, 1 \mathrm{H}), 7.74-7.61(\mathrm{~m}, 5 \mathrm{H}), 6.86(\mathrm{~d}, J=15.9 \mathrm{~Hz}, 1 \mathrm{H}), 6.63(\mathrm{brs}, 1 \mathrm{H}), 6.50-6.39(\mathrm{~m}$, $1 \mathrm{H}), 6.31-6.25(\mathrm{~m}, 1 \mathrm{H}), 5.99(\mathrm{~s}, 1 \mathrm{H}), 5.84-5.73(\mathrm{~m}, 1 \mathrm{H}), 3.37-3.31(\mathrm{~m}, 4 \mathrm{H}), 2.46-2.39(\mathrm{~m}, 4 \mathrm{H}), 2.25(\mathrm{~s}$, $3 \mathrm{H}), 2.21(\mathrm{~s}, 3 \mathrm{H})$. 


\section{Compound 4:}
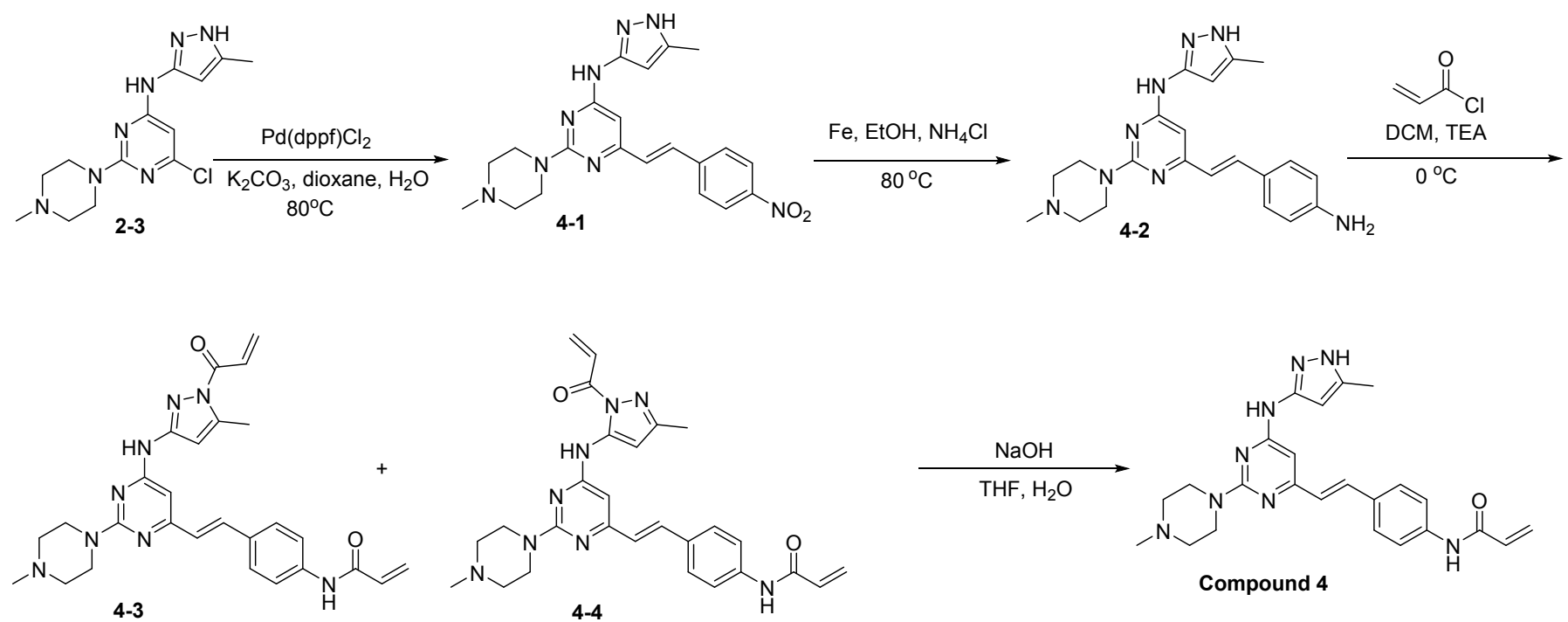<smiles>Cc1cc(Nc2cc(Cl)nc(N3CCN(C)CC3)n2)n[nH]1</smiles>

2-3<smiles>Cc1cc(Nc2cc(/C=C/c3ccc([N+](=O)[O-])cc3)nc(N3CCN(C)CC3)n2)n[nH]1</smiles>

4-1

N-(5-Methyl-1H-pyrazol-3-yl)-2-(4-methylpiperazin-1-yl)-6-[(E)-2-(4-nitrophenyl)ethenyl]pyrimidin-4amine (4-1): A mixture of 6-chloro- $\mathrm{N}$-(5-methyl-1H-pyrazol-3-yl)-2-(4-methylpiperazin-1-yl)pyrimidin-4amine (200 mg, $0.650 \mathrm{mmol}, 1$ equiv), 4,4,5,5-tetramethyl-2-[(E)-2-(4-nitrophenyl)ethenyl]-1,3,2dioxaborolane (268.15 mg, $0.975 \mathrm{mmol}, 1.5$ equiv), $\mathrm{Pd}(\mathrm{dppf}) \mathrm{Cl}_{2}$ (95.1 mg, $0.130 \mathrm{mmol}, 0.2$ equiv) and $\mathrm{K}_{2} \mathrm{CO}_{3}\left(179.61 \mathrm{mg}, 1.300 \mathrm{mmol}, 2\right.$ equiv) in dioxane $(6 \mathrm{~mL})$ and $\mathrm{H}_{2} \mathrm{O}(0.6 \mathrm{~mL})$ was stirred at $80^{\circ} \mathrm{C}$ overnight under nitrogen atmosphere. The resulting mixture was concentrated under vacuum. The residue was purified by silica gel column chromatography, eluted with $\mathrm{CH}_{2} \mathrm{Cl}_{2} / \mathrm{MeOH}(4 / 1)$ to afford $\mathrm{N}$-(5-methyl-1Hpyrazol-3-yl)-2-(4-methylpiperazin-1-yl)-6-[(E)-2-(4-nitrophenyl)ethenyl]pyrimidin-4-amine (200 mg, 73\%) as a yellow solid. LCMS (ESI): [M+H] $]^{+}$421.2. 
<smiles>Cc1cc(Nc2cc(/C=C/c3ccc([N+](=O)[O-])cc3)nc(N3CCN(C)CC3)n2)nc(Nc2cc(/C=C/c3ccc(N)cc3)nc(N3CCN(C)CC3)n2)n1</smiles>

6-[(E)-2-(4-Aminophenyl)ethenyl]-N-(5-methyl-1H-pyrazol-3-yl)-2-(4-methylpiperazin-1-yl)pyrimidin-4amine (4-2): A mixture of $N$-(5-methyl-1H-pyrazol-3-yl)-2-(4-methylpiperazin-1-yl)-6-[(E)-2-(4nitrophenyl)ethenyl]pyrimidin-4-amine ( $200 \mathrm{mg}, 0.476 \mathrm{mmol}, 1$ equiv), iron (132.8 mg, $2.378 \mathrm{mmol} 5$ equiv) and $\mathrm{NH}_{4} \mathrm{Cl}\left(254.4 \mathrm{mg}, 4.757 \mathrm{mmol}, 10\right.$ equiv) in $\mathrm{EtOH}(10 \mathrm{~mL})$ and $\mathrm{H}_{2} \mathrm{O}(1 \mathrm{ml})$ was stirred at $80{ }^{\circ} \mathrm{C}$ for 2 hours. The resulting mixture was filtered. The filtrate was concentrated under reduced pressure. This resulted in 6-[(E)-2-(4-aminophenyl)ethenyl]- $N$-(5-methyl-1H-pyrazol-3-yl)-2-(4-methylpiperazin-1yl)pyrimidin-4-amine (200 mg) as a yellow crude solid. LCMS (ESI): [M+H]+: 391.2.

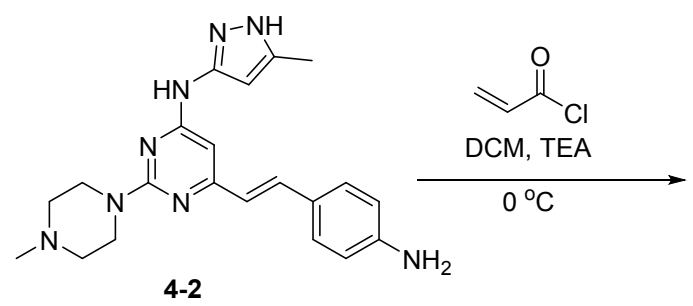

4-2

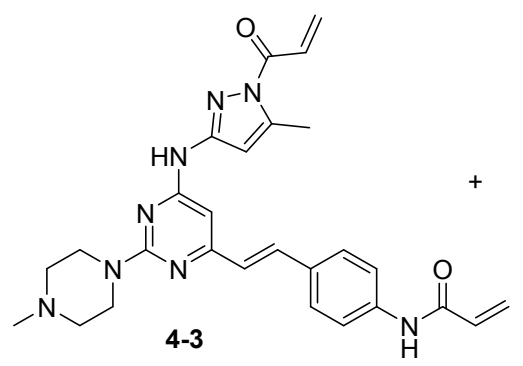

4-3

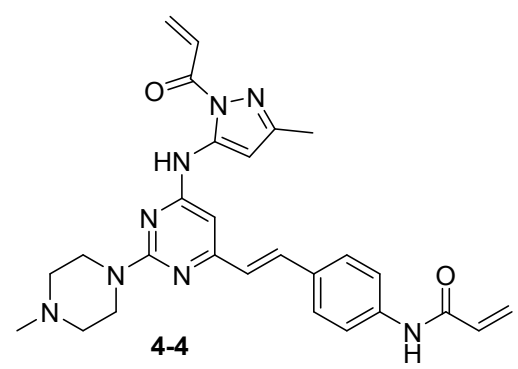

4-4

$N$-[4-[(E)-2-(6-[[5-Methyl-1-(prop-2-enoyl)-1H-pyrazol-3-yl]amino]-2-(4-methylpiperazin-1yl)pyrimidin-4-yl)ethenyl]phenyl]prop-2-enamide (4-3) and (E)- $\mathrm{N}$-(4-(2-(6-(1-Acryloyl-3-methyl-1Hpyrazol-5-ylamino)-2-(4-methylpiperazin-1-yl)pyrimidin-4-yl)vinyl)phenyl)acrylamide (4-4): To a solution of 6-[(E)-2-(4-aminophenyl)ethenyl]- $N$-(5-methyl-1H-pyrazol-3-yl)-2-(4-methylpiperazin-1yl)pyrimidin-4-amine (150 mg, $0.384 \mathrm{mmol}, 1$ equiv) in DCM (4 mL) was added prop-2-enoyl chloride (69.5 $\mathrm{mg}, 0.768 \mathrm{mmol}, 2$ equiv) and diisopropylethylamine ( $99.3 \mathrm{mg}, 0.768 \mathrm{mmol}, 2$ equiv) at $0{ }^{\circ} \mathrm{C}$. The resulting mixture was stirred at room temperature for 1 hour. The reaction was quenched with saturate aqueous $\mathrm{NaHCO}_{3}$ at room temperature. The aqueous layer was extracted with $\mathrm{CH}_{2} \mathrm{Cl}_{2}$. The combined organic layers were dried with sodium sulfate and concentrated under vacuum. The residue was purified by Prep-TLC eluting with $\mathrm{CH}_{2} \mathrm{Cl}_{2} / \mathrm{MeOH}(15 / 1)$ to afford a mixture of $N$-[4-[(E)-2-(6-[[5-methyl-1-(prop-2-enoyl)- $1 H$ pyrazol-3-yl]amino]-2-(4-methylpiperazin-1-yl)pyrimidin-4-yl)ethenyl]phenyl]prop-2-enamide and $(E)-N$ (4-(2-(6-(1-acryloyl-3-methyl-1H-pyrazol-5-ylamino)-2-(4-methylpiperazin-1-yl)pyrimidin-4yl)vinyl)phenyl)acrylamide (60 mg, 31\%) as a light yellow solid. LCMS (ESI): [M+H]+: 499.2. 


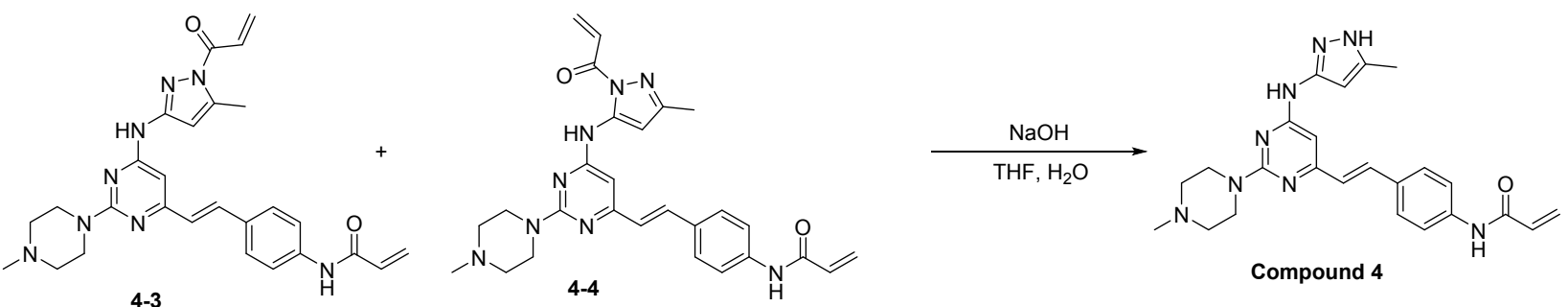

(E)-N-(4-(2-(6-(5-Methyl-1H-pyrazol-3-ylamino)-2-(4-methylpiperazin-1-yl)pyrimidin-4-

yl)vinyl)phenyl)acrylamide (Compound 4): To a stirred solution of $\mathrm{N}$-[4-[(E)-2-(6-[[5-methyl-1-(prop-2enoyl)-1H-pyrazol-3-yl]amino]-2-(4-methylpiperazin-1-yl)pyrimidin-4-yl)ethenyl]phenyl]prop-2-enamide and (E)-N-(4-(2-(6-(1-acryloyl-3-methyl-1H-pyrazol-5-ylamino)-2-(4-methylpiperazin-1-yl)pyrimidin-4yl)vinyl)phenyl)acrylamide (40 mg, 0.080 mmol, 1 equiv) in THF (1 mL) and $\mathrm{H}_{2} \mathrm{O}(1 \mathrm{~mL})$ were added $\mathrm{NaOH}$ (3.21 mg, $0.080 \mathrm{mmol}, 1$ equiv) at room temperature. The resulting mixture was stirred at room temperature for 30 minutes. The crude product was purified by Prep-HPLC with following conditions: Column: XBridge Prep OBD C18 Column 30×150 mm 5 um; Mobile Phase A: Water (10 mmol/ $\mathrm{L} \mathrm{NH}_{4} \mathrm{HCO}_{3}$ ), Mobile Phase B: ACN; Flow rate: 60 mL/min; Gradient: 24\% B to 42\% B in 7 min; 254/220 nm; Rt: 6.25 min. This resulted in (E)-N-(4-(2-(6-(5-methyl-1H-pyrazol-3-ylamino)-2-(4-methylpiperazin-1-yl)pyrimidin-4yl)vinyl)phenyl)acrylamide (13 mg, 37\%) as a light yellow solid. LCMS (ESI): [M+H] ${ }^{+}: 445.2 .{ }^{1} \mathrm{H}$ NMR (300 $\left.\mathrm{MHz}, \mathrm{DMSO}-d_{6}\right) \delta 11.90(\mathrm{~s}, 1 \mathrm{H}), 10.26(\mathrm{~s}, 1 \mathrm{H}), 9.41(\mathrm{~s}, 1 \mathrm{H}), 7.71-7.56(\mathrm{~m}, 5 \mathrm{H}), 6.90(\mathrm{~d}, J=15.7 \mathrm{~Hz}, 1 \mathrm{H})$, $6.49-6.30(\mathrm{~m}, 3 \mathrm{H}), 6.16(\mathrm{brs}, 1 \mathrm{H}), 5.79-5.75(\mathrm{~m}, 1 \mathrm{H}), 3.79-3.72(\mathrm{~m}, 4 \mathrm{H}), 2.43-2.34(\mathrm{~m}, 4 \mathrm{H}), 2.26-$ $2.11(\mathrm{~m}, 6 \mathrm{H})$.

\section{Compound 5:}
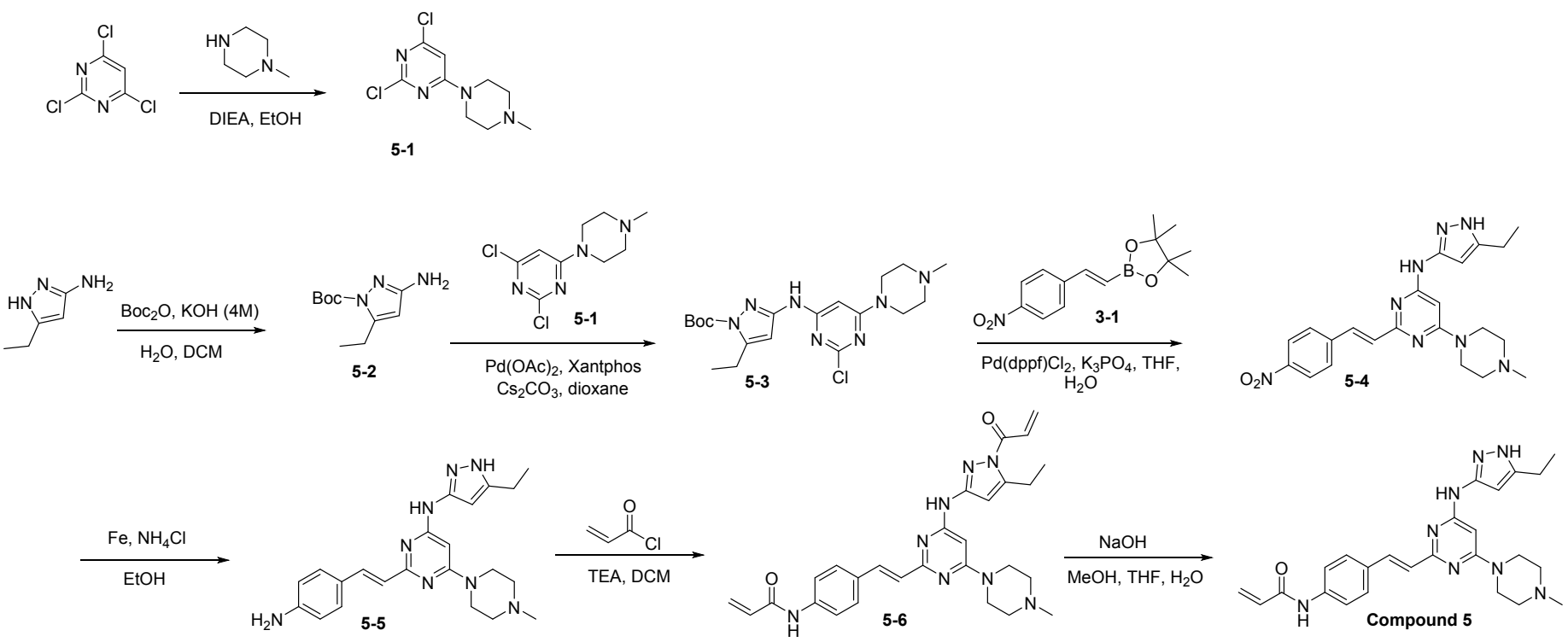
<smiles>Clc1cc(Cl)nc(Cl)n1</smiles><smiles>CCOc1ccccc1</smiles><smiles>CN1CCN(c2cc(Cl)nc(Cl)n2)CC1</smiles>

\section{5-1}

2, 4-Dichloro-6-(4-methylpiperazin-1-yl)pyrimidine (5-1): To a mixture of 2,4,6-trichloropyrimidine (10 g, $54.52 \mathrm{mmol}, 1$ equiv) and diisopropylethylamine ( $21.14 \mathrm{~g}, 163.559 \mathrm{mmol}, 3$ equiv) in EtOH (100 mL) were added 1-methyl piperazine $\left(5.46 \mathrm{~g}, 54.52 \mathrm{mmol}, 1\right.$ equiv) dropwise at $-30^{\circ} \mathrm{C}$. The resulting mixture was stirred at $-30^{\circ} \mathrm{C}$ for 4 hours. The reaction was concentrated under vacuum. The residue was purified by C18 reverse flash chromatography eluting with $\mathrm{MeCN} / \mathrm{H}_{2} \mathrm{O}$ (1/1). This resulted in 2,4-dichloro-6-(4methylpiperazin-1-yl)pyrimidine $(7.1 \mathrm{~g}, 53 \%)$ as a white solid. LCMS (ESI): $[\mathrm{M}+\mathrm{H}]^{+}:$247.0. Structure was assigned by $1 \mathrm{H}$ NMR analysis after de-chlorination.
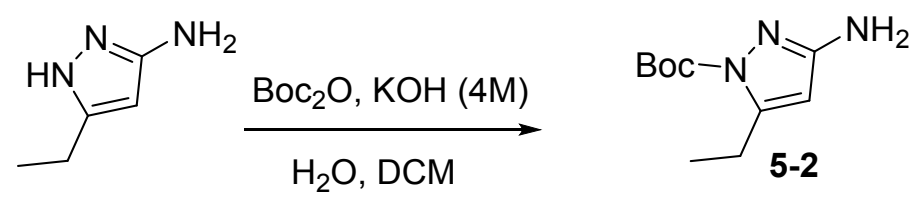

tert-Butyl 3-amino-5-ethylpyrazole-1-carboxylate (5-2): To a mixture of 5-ethyl-1H-pyrazol-3-amine (2 g, $17.994 \mathrm{mmol}, 1$ equiv) in $\mathrm{DCM}(20 \mathrm{~mL})$ were added $\mathrm{KOH}\left(8.1 \mathrm{~g}, 143.952 \mathrm{mmol}, 8\right.$ equiv) in $\mathrm{H}_{2} \mathrm{O}(36 \mathrm{~mL})$ and $\mathrm{Boc}_{2} \mathrm{O}$ ( $4.7 \mathrm{~g}, 21.581 \mathrm{mmol}, 1.2$ equiv). The resulting mixture was stirred at room temperature for 3 $\mathrm{h}$. The resulting mixture was extracted with DCM. The resulting mixture was concentrated under vacuum. The residue was purified by silica gel column chromatography, eluted with PE/EtOAc (5/1) to afford tertbutyl 3-amino-5-ethylpyrazole-1-carboxylate ( $3 \mathrm{~g}, 79 \%)$ as a white solid. LCMS (ESI): $[\mathrm{M}+\mathrm{H}]^{+}:$: $^{212.3 .^{1}}$<smiles>CCc1cc(N)nn1C(=O)OCc1cc(Nc2cc(N3CCN(C)CC3)nc(Cl)n2)nn1C(=O)OC(C)(C)C</smiles>

tert-Butyl 3-[[2-chloro-6-(4-methylpiperazin-1-yl)pyrimidin-4-yl]amino]-5-ethylpyrazole-1-carboxylate (5-3): To a mixture of tert-butyl 3-amino-5-ethylpyrazole-1-carboxylate (400 mg, $1.893 \mathrm{mmol}, 1$ equiv) and 2,4-dichloro-6-(4-methylpiperazin-1-yl)pyrimidine (701 mg, $2.840 \mathrm{mmol}, 1.5$ equiv) in 1,4-dioxane (20 mL) were added $\mathrm{Pd}(\mathrm{OAc})_{2}(85 \mathrm{mg}, 0.379 \mathrm{mmol}, 0.2$ equiv), Xantphos (219 mg, $0.379 \mathrm{mmol}, 0.2$ equiv) and

\footnotetext{
${ }^{1}$ Boc protections on aminopyrazoles typically give inseperable mixtures of 1-N and 2-N isomers and are pushed forward as such.
} 
$\mathrm{Cs}_{2} \mathrm{CO}_{3}$ (801 mg, $2.461 \mathrm{mmol}, 1.3$ equiv). The resulting mixture was stirred at $35{ }^{\circ} \mathrm{C}$ overnight under a nitrogen atmosphere. The resulting mixture was concentrated under vacuum. The residue was purified twice, first by $\mathrm{C} 18$ reverse phase column eluting with $\mathrm{ACN} / \mathrm{H}_{2} \mathrm{O}(1 / 1)$ and then with silica gel column chromatography, eluting with $\mathrm{CH}_{2} \mathrm{Cl}_{2} / \mathrm{MeOH}$ (10/1). This resulted in mixture $(500 \mathrm{mg})$. The mixture was purified by Prep-SFC with the following conditions: Column, CHIRALPAK AD-H-TC001 SFC, $2 * 25 \mathrm{~cm}$, 5um; mobile phase, $\mathrm{A}: \mathrm{CO}_{2}$, Mobile Phase B: IPA ( $2 \mathrm{mM} \mathrm{NH}_{3}-\mathrm{MEOH}$ ); Flow rate: $40 \mathrm{~mL} / \mathrm{min}$; Gradient: $20 \%$ B; 220 $\mathrm{nm}$. The resulted in tert-butyl 3-[[2-chloro-6-(4-methylpiperazin-1-yl)pyrimidin-4-yl]amino]-5ethylpyrazole-1-carboxylate (170 mg, 21\%) as a yellow oil. Structure was assigned by $1 \mathrm{H}$ NMR analysis after de-chlorination. ${ }^{2}$<smiles>CCc1cc(Nc2cc(N3CCN(C)CC3)nc(Cl)n2)nn1C(=O)OC(C)(C)C</smiles><smiles>CC1(C)OB(/C=C/c2ccc([N+](=O)[O-])cc2)OC1(C)C</smiles><smiles>CCc1cc(Nc2cc(N3CCN(C)CC3)nc(/C=C/c3ccc([N+](=O)[O-])cc3)n2)n[nH]1</smiles>

$N$-(5-Ethyl-1H-pyrazol-3-yl)-6-(4-methylpiperazin-1-yl)-2-[(E)-2-(4-nitrophenyl)ethenyl]pyrimidin-4-

amine (5-4): To a mixture of tert-butyl 3-[[2-chloro-6-(4-methylpiperazin-1-yl)pyrimidin-4-yl]amino]-5ethylpyrazole-1-carboxylate (140 mg, $0.332 \mathrm{mmol}, 1$ equiv) and 4,4,5,5-tetramethyl-2-[(E)-2-(4nitrophenyl)ethenyl]-1,3,2-dioxaborolane ( $182 \mathrm{mg}, 0.664 \mathrm{mmol}, 2$ equiv) in THF ( $5 \mathrm{~mL}$ ) were added $\mathrm{K}_{3} \mathrm{PO}_{4}$ (211 mg, $0.995 \mathrm{mmol}, 3$ equiv) in $\mathrm{H}_{2} \mathrm{O}(1 \mathrm{~mL})$ and $\mathrm{Pd}(\mathrm{dppf}) \mathrm{Cl}_{2}(72 \mathrm{mg}, 0.100 \mathrm{mmol}, 0.3$ equiv). The resulting mixture was stirred at $80{ }^{\circ} \mathrm{C}$ overnight under nitrogen atmosphere. The resulting mixture was concentrated under vacuum. The residue was purified by silica gel column chromatography, eluting with $\mathrm{CH}_{2} \mathrm{Cl}_{2} / \mathrm{MeOH}$ (10/1) to afford N-(5-ethyl-1H-pyrazol-3-yl)-6-(4-methylpiperazin-1-yl)-2-[(E)-2-(4nitrophenyl)ethenyl]pyrimidin-4-amine (100 mg, 69\%) as a brown solid. LCMS (ESI): [M+H]+: 435.2.<smiles>CCc1cc(Nc2cc(N3CCN(C)CC3)nc(/C=C/c3ccc([N+](=O)[O-])cc3)n2)n[nH]1</smiles><smiles>CCc1cc(Nc2cc(N3CCN(C)CC3)nc(/C=C/c3ccc(N)cc3)n2)n[nH]1</smiles>

2-[(E)-2-(4-Aminophenyl)ethenyl]-N-(5-ethyl-1H-pyrazol-3-yl)-6-(4-methylpiperazin-1-yl)pyrimidin-4amine (5-5): To a mixture of $N$-(5-ethyl-1H-pyrazol-3-yl)-6-(4-methylpiperazin-1-yl)-2-[(E)-2-(4-

\footnotetext{
2 For precedence of selectivity see: Koppitz, Marcus et al. PCT Int. Appl., 2019081343, 02 May 2019.
} 
nitrophenyl)ethenyl]pyrimidin-4-amine (100 mg, $0.230 \mathrm{mmol}, 1$ equiv) in EtOH ( $5 \mathrm{~mL}$ ) were added iron (104 mg, $1.870 \mathrm{mmol}, 10$ equiv) and saturated aqueous $\mathrm{NH}_{4} \mathrm{Cl}(0.5 \mathrm{~mL})$. The resulting mixture was stirred at $80{ }^{\circ} \mathrm{C}$ for $1 \mathrm{~h}$. The resulting mixture was concentrated under vacuum. The resulting mixture was dissolved in $\mathrm{MeOH}$ and filtered. The filtrate was concentrated under vacuum. This resulted in 2-[(E)-2-(4aminophenyl)ethenyl]- $N$-(5-ethyl-1H-pyrazol-3-yl)-6-(4-methylpiperazin-1-yl)pyrimidin-4-amine (50 mg, $54 \%)$ as a yellow crude solid. LCMS (ESI): $[\mathrm{M}+\mathrm{H}]^{+}: 405.4$.

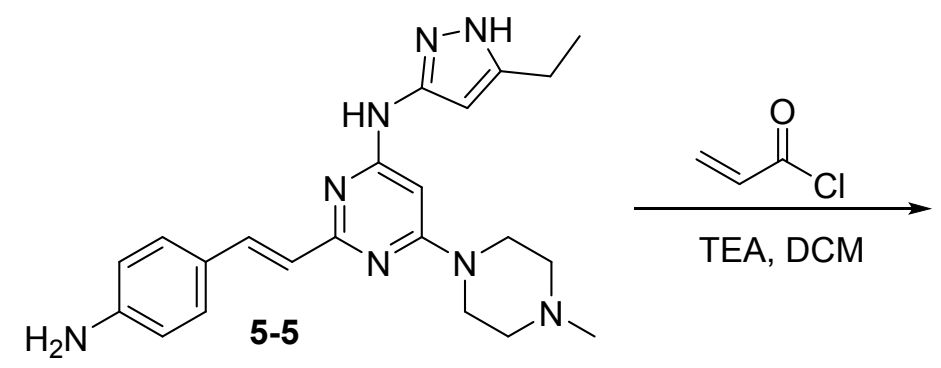<smiles>C=CC(=O)Nc1ccc(/C=C/c2nc(Nc3cc(CC)n(C(=O)C=C)n3)cc(N3CCN(C)CC3)n2)cc1</smiles>

$N$-[4-[(E)-2-(4-[[5-Ethyl-1-(prop-2-enoyl)pyrazol-3-yl]amino]-6-(4-methylpiperazin-1-yl)pyrimidin-2yl)ethenyl]phenyl]prop-2-enamide (5-6): To a mixture of 2-[(E)-2-(4-aminophenyl)ethenyl]- $N$-(5-ethyl$1 \mathrm{H}$-pyrazol-3-yl)-6-(4-methylpiperazin-1-yl)pyrimidin-4-amine (50 mg, $0.087 \mathrm{mmol}, 1$ equiv, 70\%) in DCM $(2 \mathrm{~mL}$ ) was added triethylamine ( $26 \mathrm{mg}, 0.260 \mathrm{mmol}, 3$ equiv) and acryloyl chloride $(7 \mathrm{mg}, 0.087 \mathrm{mmol}, 1$ equiv) at $0{ }^{\circ} \mathrm{C}$. The resulting mixture was stirred at $0{ }^{\circ} \mathrm{C}$ for 1 hour. The reaction was quenched with $\mathrm{MeOH}$ at $0{ }^{\circ} \mathrm{C}$. The resulting mixture was concentrated under vacuum. The residue was purified by silica gel column chromatography, eluting with $\mathrm{CH}_{2} \mathrm{Cl}_{2} / \mathrm{MeOH}$ (10/1) to afford $\mathrm{N}$-[4-[(E)-2-(4-[[5-ethyl-1-(prop-2enoyl)pyrazol-3-yl]amino]-6-(4-methylpiperazin-1-yl)pyrimidin-2-yl)ethenyl]phenyl]prop-2-enamide (40 $\mathrm{mg}, 63 \%)$ as a yellow solid.<smiles>C=CC(=O)Nc1ccc(/C=C/c2nc(Nc3cc(CC)n(C(=O)C=C)n3)cc(N3CCN(C)CC3)n2)cc1</smiles><smiles>C=CC(=O)Nc1ccc(/C=C/c2nc(Nc3cc(CC)[nH]n3)cc(N3CCN(C)CC3)n2)cc1</smiles>

$N$-[4-[(E)-2-[4-[(5-Ethyl-1H-pyrazol-3-yl)amino]-6-(4-methylpiperazin-1-yl)pyrimidin-2yl]ethenyl]phenyl]prop-2-enamide (Compound 5): To a mixture of $N$-[4-[(E)-2-(4-[[5-ethyl-1-(prop-2enoyl)pyrazol-3-yl]amino]-6-(4-methylpiperazin-1-yl)pyrimidin-2-yl)ethenyl]phenyl]prop-2-enamide (40 $\mathrm{mg}, 0.055 \mathrm{mmol}, 1$ equiv, $70 \%)$ in $\mathrm{MeOH}(1 \mathrm{~mL})$, THF $(1 \mathrm{~mL})$ and $\mathrm{H}_{2} \mathrm{O}(1 \mathrm{~mL})$ were added $\mathrm{NaOH}(4 \mathrm{mg}, 0.109$ 
mmol, 2 equiv). The resulting mixture was stirred at room temperature for 1 hour. The resulting mixture was concentrated under vacuum. The crude product was purified by Prep-HPLC with the following conditions: Column, XBridge Prep OBD C18 Column, 30×150mm 5um; mobile phase, Water with 10mmol $\mathrm{NH}_{4} \mathrm{HCO}_{3}$ and $\mathrm{MeCN}$ (25.0\% MeCN up to 55.0\% in $\left.7 \mathrm{~min}\right) ;$ UV $254 \mathrm{~nm}$. This resulted in N-[4-[(E)-2-[4-[(5ethyl-1H-pyrazol-3-yl)amino]-6-(4-methylpiperazin-1-yl)pyrimidin-2-yl]ethenyl]phenyl]prop-2-enamide (1.4 mg, 6\%) as a light yellow solid. LCMS (ESI): $[\mathrm{M}+\mathrm{H}]^{+}:$459.4. ${ }^{1} \mathrm{H} \mathrm{NMR}\left(300 \mathrm{MHz}, \mathrm{CD}_{3} \mathrm{COCD}_{3}\right) \delta 9.49$ (s, $1 \mathrm{H}), 8.31(\mathrm{~s}, 1 \mathrm{H}), 7.86-7.74(\mathrm{~m}, 3 \mathrm{H}), 7.63-7.54(\mathrm{~m}, 2 \mathrm{H}), 6.97-6.86(\mathrm{~m}, 1 \mathrm{H}), 6.72(\mathrm{~s}, 1 \mathrm{H}), 6.54-6.29(\mathrm{~m}$, $2 \mathrm{H}), 6.07(\mathrm{~s}, 1 \mathrm{H}), 5.76-5.66(\mathrm{~m}, 1 \mathrm{H}), 3.68-3.58(\mathrm{~m}, 4 \mathrm{H}), 2.73-2.59(\mathrm{~m}, 2 \mathrm{H}), 2.49-2.40(\mathrm{~m}, 4 \mathrm{H}), 2.27(\mathrm{~s}$, $3 \mathrm{H}), 1.35-1.22(\mathrm{~m}, 4 \mathrm{H})$.

\section{Compound 6:}
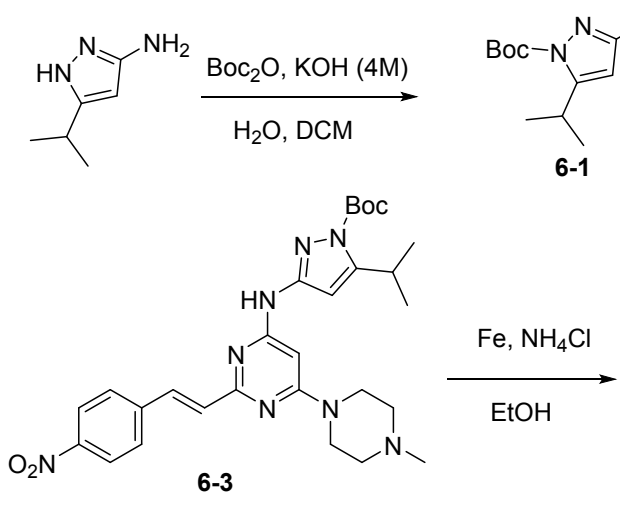

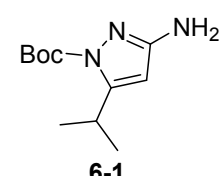

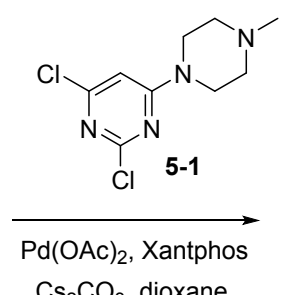

$\mathrm{Cs}_{2} \mathrm{CO}_{3}$, dioxane

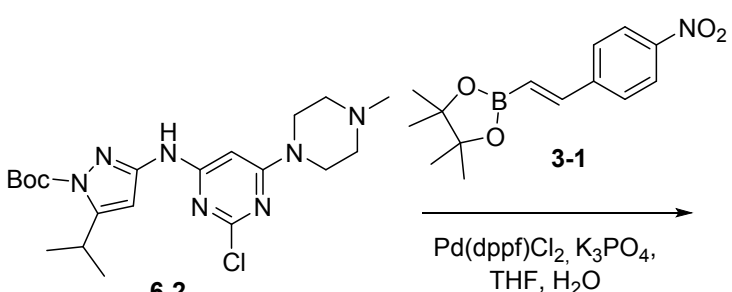

6-2 THF, $\mathrm{H}_{2} \mathrm{O}$

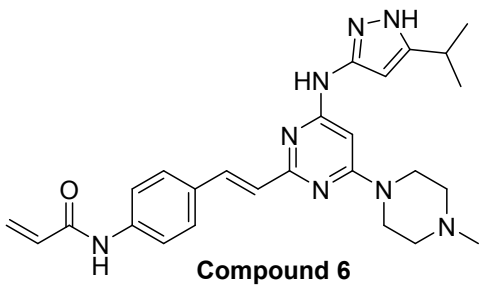<smiles>CC(C)c1cc(N)n[nH]1</smiles>

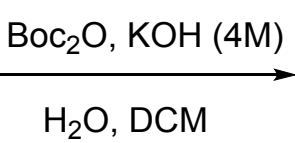<smiles>CC(C)c1cc(N)nn1C(=O)OC(C)(C)C</smiles>

6-1

tert-Butyl 3-amino-5-isopropylpyrazole-1-carboxylate (6-1): To a mixture of 5-isopropyl-1H-pyrazol-3amine (3 g, 23.966 mmol, 1 equiv) in $\mathrm{DCM}(60 \mathrm{~mL})$ and $\mathrm{H}_{2} \mathrm{O}(48 \mathrm{~mL})$ were added $\mathrm{KOH}(10.8 \mathrm{~g}, 191.732$ mmol, 8 equiv) and $\mathrm{Boc}_{2} \mathrm{O}\left(6.3 \mathrm{~g}, 28.760 \mathrm{mmol}, 1.2\right.$ equiv) at $0{ }^{\circ} \mathrm{C}$. The resulting mixture was stirred at room temperature for 2 hours. The resulting mixture was concentrated under vacuum. The residue was purified by silica gel column chromatography, eluting with $\mathrm{CH}_{2} \mathrm{Cl}_{2} / \mathrm{MeOH}(10 / 1)$. This resulted in $3 \mathrm{~g}(56 \%)$ of tert-butyl 3-amino-5-isopropylpyrazole-1-carboxylate as a yellow solid. LCMS (ESI): [M+H]+: 226.1 . 
<smiles>CC(Br)c1cc(N)nn1C(=O)OC(C)(C)C</smiles>

6-1<smiles>CN1CCN(c2cc(Cl)nc(Cl)n2)CC1</smiles>

$\mathrm{Pd}(\mathrm{OAc})_{2}$, Xantphos

$\mathrm{Cs}_{2} \mathrm{CO}_{3}$, dioxane<smiles>CC(C)c1cc(Nc2cc(N3CCN(C)CC3)nc(Cl)n2)nn1C(=O)OCc1ccccc1</smiles>

6-2

tert-Butyl

3-[[2-chloro-6-(4-methylpiperazin-1-yl)pyrimidin-4-yl]amino]-5-isopropylpyrazole-1-

carboxylate (6-2): To a mixture of tert-butyl 3-amino-5-isopropylpyrazole-1-carboxylate (720 mg, 3.196 mmol, 1 equiv) and 2,4-dichloro-6-(4-methylpiperazin-1-yl)pyrimidine (789 mg, $3.196 \mathrm{mmol}, 1$ equiv) in dioxane $\left(10 \mathrm{~mL}\right.$ ) were added $\mathrm{Cs}_{2} \mathrm{CO}_{3}(1.4 \mathrm{~g}, 4.174 \mathrm{mmol}, 1.31$ equiv), Xantphos (370 $\mathrm{mg}, 0.639 \mathrm{mmol}, 0.2$ equiv) and $\mathrm{Pd}(\mathrm{OAc})_{2}\left(5 \mathrm{mg}, 0.022 \mathrm{mmol}, 0.1\right.$ equiv). The resulting mixture was stirred at $50{ }^{\circ} \mathrm{C}$ overnight under a nitrogen atmosphere. The residue was purified by silica gel column chromatography, eluting with $\mathrm{CH}_{2} \mathrm{Cl}_{2} / \mathrm{MeOH}$ (10/1). This resulted in $550 \mathrm{mg}$ product as a mixture of two isomers. The mixture (550 $\left.\mathrm{mg}\right)$ was separated by Prep-SFC with following conditions: Column: Reg-AD, 30*250mm, 5um; Mobile Phase A: $\mathrm{CO}_{2}$, Mobile Phase B: IPA ( $2 \mathrm{mM} \mathrm{NH}_{3}-\mathrm{MEOH)}$ ) Flow rate:50 mL/min; Gradient:35\% B; $220 \mathrm{~nm}$; RT1:5.41; RT2:7.85; Injection Volumn:1.5 ml; Number of Runs:17. This resulted in 220 mg (16\%) of tert-butyl 3-[[2chloro-6-(4-methylpiperazin-1-yl)pyrimidin-4-yl]amino]-5-isopropylpyrazole-1-carboxylate as a light yellow solid. $\mathrm{LCMS}(\mathrm{ESI})$ : $[\mathrm{M}+\mathrm{H}]^{+}: 436.2$. Structures were assigned by $1 \mathrm{H}$ NMR analysis after de-chlorination.<smiles>CC(C)c1cc(Nc2cc(N3CCN(C)CC3)nc(/C=C/c3ccc([N+](=O)[O-])cc3)n2)nc(Nc2cc(N3CCN(C)CC3)nc(Cl)n2)n1</smiles>

tert-Butyl 5-isopropyl-3-[[6-(4-methylpiperazin-1-yl)-2-[(E)-2-(4-nitrophenyl)ethenyl]pyrimidin-4yl]amino]pyrazole-1-carboxylate (6-3): To a mixture of tert-butyl 3-[[2-chloro-6-(4-methylpiperazin-1yl)pyrimidin-4-yl]amino]-5-isopropylpyrazole-1-carboxylate (220 mg, $0.505 \mathrm{mmol}, 1$ equiv) and 4,4,5,5tetramethyl-2-[(E)-2-(4-nitrophenyl)ethenyl]-1,3,2-dioxaborolane (277 mg, $1.0 \mathrm{mmol}, 2$ equiv) in THF (8 $\mathrm{mL}$ ) were added $\mathrm{K}_{3} \mathrm{PO}_{4}\left(321 \mathrm{mg}, 1.514 \mathrm{mmol}, 3\right.$ equiv) and water $(0.8 \mathrm{~mL})$ with $\mathrm{Pd}(\mathrm{dppf}) \mathrm{Cl}_{2}(37 \mathrm{mg}, 0.050$ mmol, 0.1 equiv). The resulting mixture was stirred at $80^{\circ} \mathrm{C}$ overnight under nitrogen atmosphere. The resulting mixture was concentrated under vacuum. The residue was purified by C18 reverse phase column with $\mathrm{ACN} / \mathrm{H}_{2} \mathrm{O}(1 / 1)$. This resulted in $100 \mathrm{mg}(36 \%)$ of tert-butyl 5-isopropyl-3-[[6-(4-methylpiperazin-1- 
yl)-2-[(E)-2-(4-nitrophenyl)ethenyl]pyrimidin-4-yl]amino]pyrazole-1-carboxylate as a light yellow solid. LCMS (ESI): [M+H]+: 549.2 .<smiles>CCOC(=O)c1ccc(Nc2cc(N3CCN(C)CC3)nc(/C=C/c3ccc([N+](=O)[O-])cc3)n2)cc1/C=C/c1ccc(N)cc1</smiles>

2-[(E)-2-(4-Aminophenyl)ethenyl]-N-(5-isopropyl-1H-pyrazol-3-yl)-6-(4-methylpiperazin-1-yl)pyrimidin4-amine (6-4): To a mixture of tert-butyl 5-isopropyl-3-[[6-(4-methylpiperazin-1-yl)-2-[(E)-2-(4nitrophenyl)ethenyl]pyrimidin-4-yl]amino]pyrazole-1-carboxylate (100 mg, $0.182 \mathrm{mmol}, 1$ equiv) in EtOH $\left(3 \mathrm{~mL}\right.$ ) were added iron (102 mg, $1.823 \mathrm{mmol}, 10$ equiv) and saturated aqueous $\mathrm{NH}_{4} \mathrm{Cl}$ solution $(0.5 \mathrm{~mL})$. The resulting mixture was stirred at $80^{\circ} \mathrm{C}$ for $1 \mathrm{~h}$. The resulting mixture was filtered. The filtrate was concentrated under reduced pressure. This resulted in $70 \mathrm{mg}(92 \%)$ of 2-[(E)-2-(4-aminophenyl)ethenyl]$\mathrm{N}$-(5-isopropyl-1H-pyrazol-3-yl)-6-(4-methylpiperazin-1-yl)pyrimidin-4-amine as a yellow solid. LCMS (ESI): $[\mathrm{M}+\mathrm{H}]^{+}:$: 419.2 .

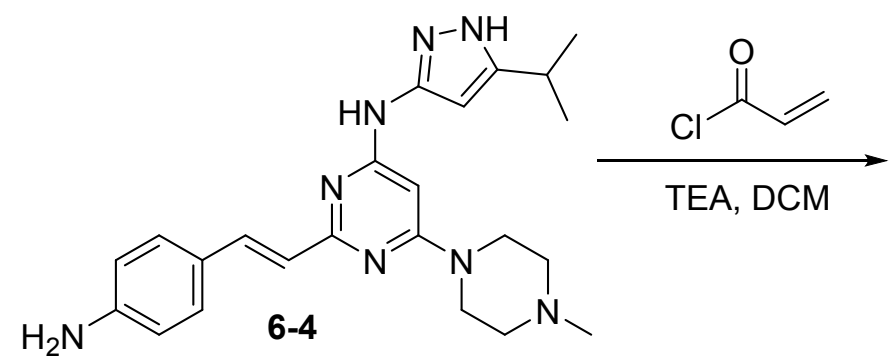<smiles>C=CC(=O)Nc1ccc(/C=C/c2nc(Nc3cc(C(C)C)[nH]n3)cc(N3CCN(C)CC3)n2)cc1</smiles>

$N$-[4-[(E)-2-[4-[(5-isopropyl-1H-pyrazol-3-yl)amino]-6-(4-methylpiperazin-1-yl)pyrimidin-2-

yl]ethenyl]phenyl]prop-2-enamide (Compound 6): To a mixture of 2-[(E)-2-(4-aminophenyl)ethenyl]- $N$ (5-isopropyl-1H-pyrazol-3-yl)-6-(4-methylpiperazin-1-yl)pyrimidin-4-amine (70 mg, $0.167 \mathrm{mmol}, 1$ equiv) in DCM (5 mL) were added triethylamine $(51 \mathrm{mg}, 0.5 \mathrm{mmol}, 3$ equiv) and acryloyl chloride (17 mg, 0.184 mmol, 1.1 equiv) dropwise at $0{ }^{\circ} \mathrm{C}$. The resulting mixture was stirred at $0{ }^{\circ} \mathrm{C}$ for 1 hour. The reaction was quenched by the addition of $\mathrm{MeOH}(2 \mathrm{~mL})$. The resulting mixture was concentrated under vacuum. The residue was purified by Prep-HPLC with Column: XBridge Prep OBD C18 Column, 30×150mm 5um; Mobile Phase A: Water (10MMOL/L NH $\mathrm{HCO}_{3}$ ), Mobile Phase B: ACN; Flow rate: $60 \mathrm{~mL} / \mathrm{min}$; Gradient: 25 B to 50 $\mathrm{B}$ in $7 \mathrm{~min} ; 254 \mathrm{~nm}$; RT1: 6.68. This resulted in $16.8 \mathrm{mg}$ (21\%) of $\mathrm{N}$-[4-[(E)-2-[4-[(5-isopropyl-1H-pyrazol-3yl)amino]-6-(4-methylpiperazin-1-yl)pyrimidin-2-yl]ethenyl]phenyl]prop-2-enamide as a yellow solid. LCMS (ESI): $[\mathrm{M}+\mathrm{H}]^{+}:$473.2. ${ }^{1} \mathrm{H}$ NMR (300 MHz, DMSO-d 6 ) $\delta 11.90(\mathrm{~s}, 1 \mathrm{H}), 10.25(\mathrm{~s}, 1 \mathrm{H}), 9.17(\mathrm{~s}, 1 \mathrm{H}), 7.75-$ 
$7.63(\mathrm{~m}, 3 \mathrm{H}), 7.59(\mathrm{~d}, J=8.4 \mathrm{~Hz}, 2 \mathrm{H}), 6.86-6.83(\mathrm{~m}, 1 \mathrm{H}), 6.69(\mathrm{~s}, 1 \mathrm{H}), 6.51-6.36(\mathrm{~m}, 1 \mathrm{H}), 6.32-6.20(\mathrm{~m}$, $1 \mathrm{H}), 5.97(\mathrm{~s}, 1 \mathrm{H}), 5.81-5.71(\mathrm{~m}, 1 \mathrm{H}), 3.52(\mathrm{~s}, 4 \mathrm{H}), 2.96-2.81(\mathrm{~m}, 1 \mathrm{H}), 2.38(\mathrm{~s}, 4 \mathrm{H}), 2.21(\mathrm{~s}, 3 \mathrm{H}), 1.21(\mathrm{~d}, J$ $=6.9 \mathrm{~Hz}, 6 \mathrm{H})$.

\section{Compound 7:}
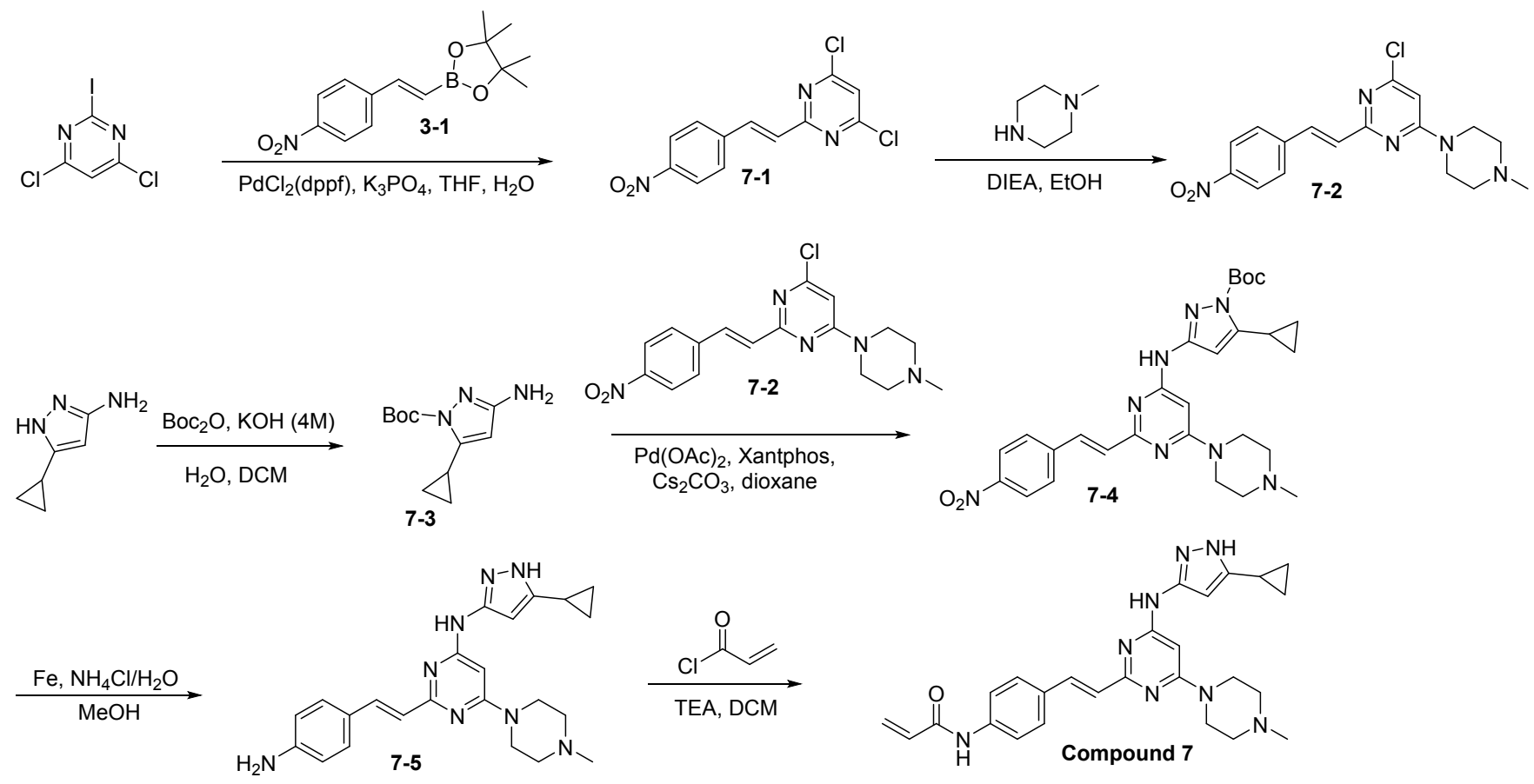<smiles>Clc1cc(Cl)nc(I)n1</smiles><smiles>CC1(C)OB(/C=C/c2ccc([N+](=O)[O-])cc2)OC1(C)C</smiles><smiles>O=[N+]([O-])c1ccc(/C=C/c2nc(Cl)cc(Cl)n2)cc1</smiles>

4,6-Dichloro-2-[(E)-2-(4-nitrophenyl)ethenyl]pyrimidine (7-1): To a mixture of 4,6-dichloro-2iodopyrimidine ( $2 \mathrm{~g}, 7.3 \mathrm{mmol}, 1$ equiv) and 4,4,5,5-tetramethyl-2-[(E)-2-(4-nitrophenyl)ethenyl]-1,3,2dioxaborolane (2 g, $7.3 \mathrm{mmol}, 1$ equiv) in THF $\left(25 \mathrm{~mL}\right.$ ) were added $\mathrm{K}_{3} \mathrm{PO}_{4}(4.6 \mathrm{~g}, 21.8 \mathrm{mmol}, 3$ equiv) in $\mathrm{H}_{2} \mathrm{O}(2.5 \mathrm{~mL})$ and $\mathrm{Pd}(\mathrm{dppf}) \mathrm{Cl}_{2}\left(0.53 \mathrm{~g}, 0.73 \mathrm{mmol}, 0.1\right.$ equiv). The resulting mixture was stirred at $80{ }^{\circ} \mathrm{C}$ overnight under nitrogen. The resulting mixture was concentrated under vacuum. The residue was applied onto a silica gel column with ethyl acetate/petroleum ether (1/5). This resulted in $1.4 \mathrm{~g}(61 \%)$ of 4,6dichloro-2-[(E)-2-(4-nitrophenyl)ethenyl]pyrimidine as a yellow solid. LCMS (ESI): [M+H]+: 296.0. 
<smiles>O=[N+]([O-])c1ccc(/C=C/c2nc(Cl)cc(Cl)n2)cc1</smiles><smiles>CCOCCNCCO</smiles><smiles>CN1CCN(c2cc(Cl)nc(/C=C/c3ccc([N+](=O)[O-])cc3)n2)CC1</smiles>

4-Chloro-6-(4-methylpiperazin-1-yl)-2-[(E)-2-(4-nitrophenyl)ethenyl]pyrimidine (7-2): To a mixture of 4,6-dichloro-2-[(E)-2-(4-nitrophenyl)ethenyl]pyrimidine ( $800 \mathrm{mg}, 2.7 \mathrm{mmol}, 1$ equiv) in $\mathrm{EtOH}(10 \mathrm{~mL}$ ) was added 1-methylpiperazine ( $271 \mathrm{mg}, 2.7 \mathrm{mmol}, 1$ equiv) and diisopropylethylamine (1.05 g, $8.1 \mathrm{mmol}, 3$ equiv). The resulting mixture was stirred at room temperature for 5 hours. The resulting mixture was concentrated under vacuum. The residue was applied onto a silica gel column with $\mathrm{CH}_{2} \mathrm{Cl}_{2} / \mathrm{MeOH}(20 / 1)$. This resulted in $700 \mathrm{mg} \quad(69 \%)$ of 4-chloro-6-(4-methylpiperazin-1-yl)-2-[(E)-2-(4nitrophenyl)ethenyl]pyrimidine as a yellow solid. LCMS (ESI): [M+H]+: 360.1.
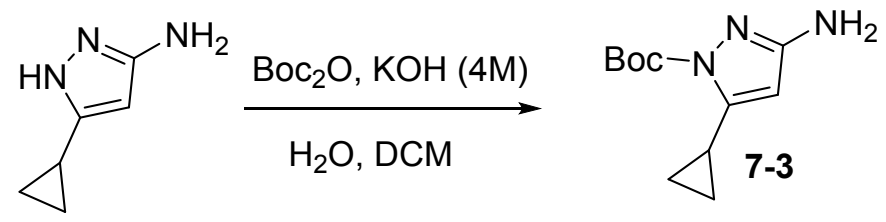

tert-Butyl 3-amino-5-cyclopropylpyrazole-1-carboxylate (7-3): To a mixture of 5-cyclopropyl-1H-pyrazol3-amine ( $3 \mathrm{~g}, 24.359 \mathrm{mmol}, 1$ equiv) and $\mathrm{KOH}\left(10.93 \mathrm{~g}, 0.195 \mathrm{mmol}, 8\right.$ equiv) in DCM (40 mL) and $\mathrm{H}_{2} \mathrm{O}$ $(48.7 \mathrm{~mL})$ was added $\mathrm{Boc}_{2} \mathrm{O}\left(6.38 \mathrm{~g}, 0.029 \mathrm{mmol}, 1.2\right.$ equiv) at $0{ }^{\circ} \mathrm{C}$. The resulting mixture was stirred at room temperature overnight. The resulting mixture was concentrated under reduced pressure. The residue was applied onto a silica gel column with PE/EA (1/1). This resulted in $4.2 \mathrm{~g}$ (77\%) of tert-butyl 3amino-5-cyclopropylpyrazole-1-carboxylate as a yellow solid. LCMS (ESI): [M+H] $]^{+}: 224.1$.<smiles>[Y6]C=Cc1nc(Nc2cc(C3CC3)n(C(=O)OC(C)(C)C)n2)cc(N2CCN(C)CC2)n1</smiles>

tert-Butyl 5-cyclopropyl-3-[[6-(4-methylpiperazin-1-yl)-2-[(E)-2-(4-nitrophenyl)ethenyl]pyrimidin-4yl]amino]pyrazole-1-carboxylate (7-4): To a mixture of 4-chloro-6-(4-methylpiperazin-1-yl)-2-[(E)-2-(4nitrophenyl)ethenyl]pyrimidine (300 $\mathrm{mg}, 0.834 \mathrm{mmol}, 1$ equiv) and tert-butyl 3-amino-5cyclopropylpyrazole-1-carboxylate (186 $\mathrm{mg}, 0.834 \mathrm{mmol}, 1$ equiv) in dioxane $\left(5 \mathrm{~mL}\right.$ ) were added $\mathrm{Cs}_{2} \mathrm{CO}_{3}$ (353 mg, $1.084 \mathrm{mmol}, 1.3$ equiv), Xantphos (97 mg, $0.167 \mathrm{mmol}, 0.2$ equiv) and $\mathrm{Pd}(\mathrm{OAc})_{2}$ (19 mg, 0.083 
mmol, 0.1 equiv). The resulting mixture was stirred at $80{ }^{\circ} \mathrm{C}$ for 4 hours. The resulting mixture was concentrated under vacuum. The residue was applied onto a silica gel column eluting with dichloromethane/methanol (10/1). This resulted in 200 mg (44\%) of tert-butyl 5-cyclopropyl-3-[[6-(4methylpiperazin-1-yl)-2-[(E)-2-(4-nitrophenyl)ethenyl]pyrimidin-4-yl]amino]pyrazole-1-carboxylate as a yellow solid. LCMS (ESI): [M+H]+: 547.3.<smiles>CN1CCN(c2cc(Nc3cc(C4CC4)n(C(=O)OC(C)(C)C)n3)nc(/C=C/c3ccc([N+](=O)[O-])cc3)n2)CC1</smiles>

7-4

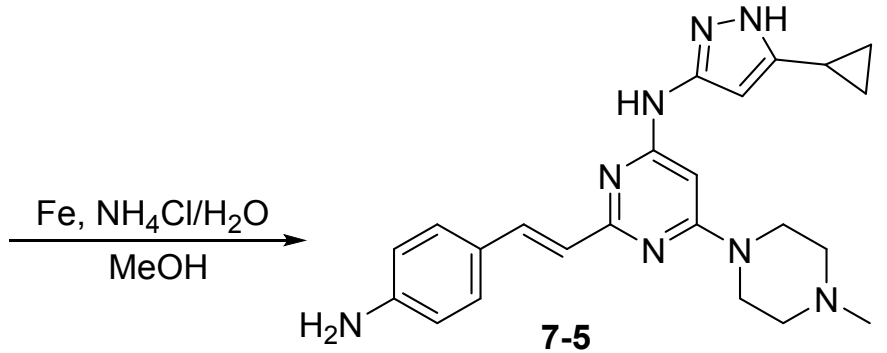

7-5

2-[(E)-2-(4-Aminophenyl)ethenyl]-N-(5-cyclopropyl-1H-pyrazol-3-yl)-6-(4-methylpiperazin-1-

yl)pyrimidin-4-amine (7-5): To a mixture of tert-butyl 5-cyclopropyl-3-[[6-(4-methylpiperazin-1-yl)-2-[(E)2-(4-nitrophenyl)ethenyl]pyrimidin-4-yl]amino]pyrazole-1-carboxylate (180 mg, $0.329 \mathrm{mmol}, 1$ equiv) in $\mathrm{MeOH}\left(5 \mathrm{~mL}\right.$ ) was added iron (185 mg, $3.293 \mathrm{mmol}, 10$ equiv) and saturated $\mathrm{NH}_{4} \mathrm{Cl}$ solution ( $1 \mathrm{~mL}$ ). The resulting mixture was stirred at $80^{\circ} \mathrm{C}$ for 1 hour. The resulting mixture was concentrated under vacuum. The residue was applied onto a silica gel column with dichloromethane/methanol (5/1). This resulted in $90 \mathrm{mg}$ (66\%) of 2-[(E)-2-(4-aminophenyl)ethenyl]- $N$-(5-cyclopropyl-1H-pyrazol-3-yl)-6-(4-methylpiperazin1-yl)pyrimidin-4-amine as a yellow solid. LCMS (ESI) [M+H] $]^{+}$417.3.<smiles>CN1CCN(c2cc(Nc3cc(C4CC4)[nH]n3)nc(/C=C/c3ccc(N)cc3)n2)CC1</smiles>

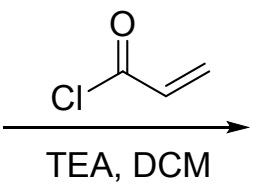<smiles>C=CC(=O)Nc1ccc(/C=C/c2nc(Nc3cc(C4CC4)[nH]n3)cc(N3CCN(C)CC3)n2)cc1</smiles>

$N$-[4-[(E)-2-[4-[(5-cyclopropyl-1H-pyrazol-3-yl)amino]-6-(4-methylpiperazin-1-yl)pyrimidin-2yl]ethenyl]phenyl]prop-2-enamide (Compound 7): To a mixture of 2-[(E)-2-(4-aminophenyl)ethenyl]- $N$ (5-cyclopropyl-1H-pyrazol-3-yl)-6-(4-methylpiperazin-1-yl)pyrimidin-4-amine (70 mg, $0.168 \mathrm{mmol}, 1$ equiv) in DCM ( $5 \mathrm{~mL}$ ) was added triethylamine $(51 \mathrm{mg}, 0.504 \mathrm{mmol}, 3$ equiv) and acryloyl chloride (18 $\mathrm{mg}, 0.202$ mmol, 1.2 equiv). The resulting mixture was stirred at $0{ }^{\circ} \mathrm{C}$ for 1 hour. The reaction was quenched with $\mathrm{MeOH}$. The resulting mixture was concentrated under vacuum. The crude product was purified by PrepHPLC with the following conditions: Column, Triart Diol-NP, 20*250 mm,5 um; Mobile Phase A: CO2, Mobile Phase B: $\mathrm{MeOH}\left(8 \mathrm{mmol} / \mathrm{L} \mathrm{NH}_{3} . \mathrm{MeOH}\right.$ )--HPLC; Flow rate: $50 \mathrm{~mL} / \mathrm{min}$; Gradient: $40 \%$ B; $254 \mathrm{~nm}$. Product was obtained. This resulted in $2.3 \mathrm{mg}(3 \%)$ of $\mathrm{N}$-[4-[(E)-2-[4-[(5-cyclopropyl-1H-pyrazol-3- 
yl)amino]-6-(4-methylpiperazin-1-yl)pyrimidin-2-yl]ethenyl]phenyl]prop-2-enamide as a white solid. LCMS (ESI) $[\mathrm{M}+\mathrm{H}]^{+}$: 471.4. ${ }^{1} \mathrm{H}$ NMR (300 MHz, Methanol- $\left.d_{4}\right) \delta 7.85-7.75(\mathrm{~m}, 1 \mathrm{H}), 7.75-7.67(\mathrm{~m}, 2 \mathrm{H}), 7.65$ - $7.57(\mathrm{~m}, 2 \mathrm{H}), 7.00-6.89(\mathrm{~m}, 1 \mathrm{H}), 6.55-6.33(\mathrm{~m}, 3 \mathrm{H}), 5.97-5.91(\mathrm{~m}, 1 \mathrm{H}), 5.86-5.76(\mathrm{~m}, 1 \mathrm{H}), 3.73-$ $3.67(\mathrm{~m}, 4 \mathrm{H}), 2.61-2.52(\mathrm{~m}, 4 \mathrm{H}), 2.37(\mathrm{~s}, 3 \mathrm{H}), 2.01-1.86(\mathrm{~m}, 1 \mathrm{H}), 1.05-0.96(\mathrm{~m}, 2 \mathrm{H}), 0.81-0.72(\mathrm{~m}, 2 \mathrm{H})$.

\section{Compound 8:}

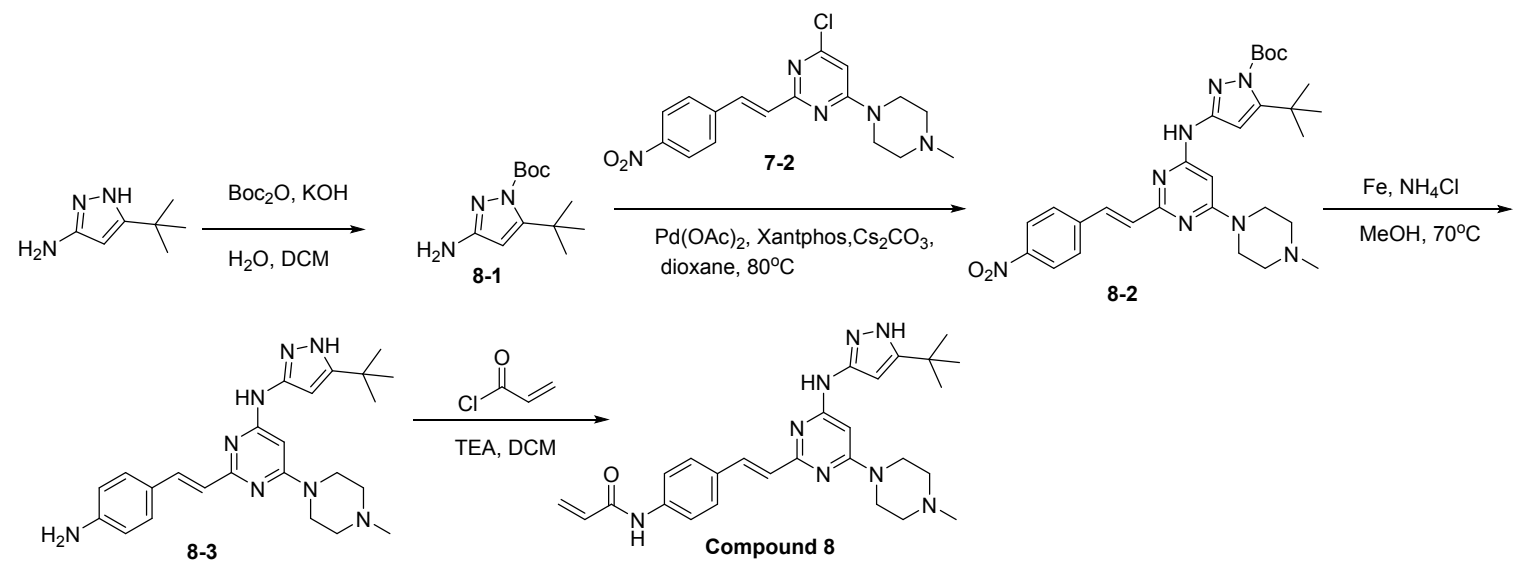<smiles>CC(C)(C)c1cc(N)n[nH]1</smiles>

tert-Butyl 3-amino-5-tert-butylpyrazole-1-carboxylate (8-1): To a mixture of 5-tert-butyl- $\mathrm{H}$-pyrazol-3amine ( $3 \mathrm{~g}, 21.55 \mathrm{mmol}, 1$ equiv) in DCM (60 mL) were added $\mathrm{KOH}\left(9.7 \mathrm{~g}, 172.41 \mathrm{mmol}, 8\right.$ equiv) in $\mathrm{H}_{2} \mathrm{O}$ $(45 \mathrm{~mL})$ and $\mathrm{Boc}_{2} \mathrm{O}\left(5.64 \mathrm{~g}, 25.86 \mathrm{mmol}, 1.2\right.$ equiv) at $0{ }^{\circ} \mathrm{C}$. The resulting mixture was stirred at room temperature for 3 hours. The resulting mixture was extracted with $\mathrm{CH}_{2} \mathrm{Cl}_{2}$. The combined organic layers were washed with water $(3 \times 5 \mathrm{~mL})$, dried over anhydrous $\mathrm{Na}_{2} \mathrm{SO}_{4}$. After filtration, the filtrate was concentrated under reduced pressure. The residue was purified by silica gel column chromatography, eluting with PE/EA (1/1). This resulted in $2.9 \mathrm{~g}$ (51\%) of tert-butyl 3-amino-5-tert-butylpyrazole-1carboxylate as a white solid. LCMS (ESI): [M+H] $]^{+}: 240.3$. 
<smiles>CC(C)(C)OC(=O)n1nc(N)cc1C(C)(C)C</smiles>

8-1

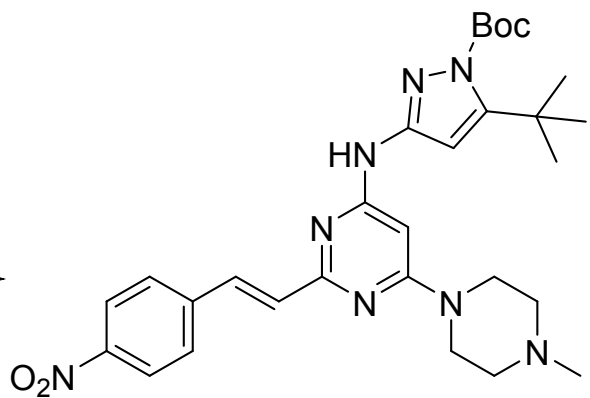

8-2

tert-Butyl 5-tert-butyl-3-[[6-(4-methylpiperazin-1-yl)-2-[(E)-2-(4-nitrophenyl)ethenyl]pyrimidin-4yl]amino]pyrazole-1-carboxylate (8-2): To a mixture of tert-butyl 3-amino-5-tert-butylpyrazole-1carboxylate (200 mg, $0.836 \mathrm{mmol}, 1$ equiv) and 4-chloro-6-(4-methylpiperazin-1-yl)-2-[(E)-2-(4nitrophenyl)ethenyl]pyrimidine ( $300 \mathrm{mg}, 0.836 \mathrm{mmol}, 1$ equiv) in dioxane $(5 \mathrm{~mL})$ were added $\mathrm{Cs}_{2} \mathrm{CO}_{3}(354$ $\mathrm{mg}, 1.086 \mathrm{mmol}, 1.3$ equiv), Xantphos ( $98 \mathrm{mg}, 0.167 \mathrm{mmol}, 0.2$ equiv) and $\mathrm{Pd}(\mathrm{OAc})_{2}(19 \mathrm{mg}, 0.084 \mathrm{mmol}$, 0.1 equiv). The resulting mixture was stirred at $80{ }^{\circ} \mathrm{C}$ for 2 hours under hydrogen atmosphere. The resulting mixture was concentrated under vacuum. The residue was purified by silica gel column chromatography, eluting with $\mathrm{CH}_{2} \mathrm{Cl}_{2} / \mathrm{MeOH}$ (10/1). This resulted in $200 \mathrm{mg}$ (40\%) of tert-butyl 5-tertbutyl-3-[[6-(4-methylpiperazin-1-yl)-2-[(E)-2-(4-nitrophenyl)ethenyl]pyrimidin-4-yl]amino]pyrazole-1carboxylate as a yellow solid. LCMS (ESI): [M+H]+: 563.2 .<smiles>COC(=O)c1ccccc1Nc1cc(N2CCN(C)CC2)nc(/C=C/c2ccc(N)cc2)n1</smiles>

2-[(E)-2-(4-Aminophenyl)ethenyl]-N-(5-tert-butyl-1H-pyrazol-3-yl)-6-(4-methylpiperazin-1yl)pyrimidin-4-amine (8-3): To a mixture of tert-butyl 5-tert-butyl-3-[[6-(4-methylpiperazin-1-yl)-2-[(E)-2(4-nitrophenyl)ethenyl]pyrimidin-4-yl]amino]pyrazole-1-carboxylate (200 mg, $0.355 \mathrm{mmol}, 1$ equiv) in ethyl alcohol (10 mL) were added iron (199 mg, $3.554 \mathrm{mmol}, 10$ equiv) and saturated aqueous $\mathrm{NH}_{4} \mathrm{Cl}(1$ $\mathrm{mL}$ ). The resulting mixture was stirred at $70{ }^{\circ} \mathrm{C}$ for 1 hour. The resulting mixture was filtered, the filter cake was washed with ethanol $(3 \times 10 \mathrm{~mL})$. The filtrate was concentrated under reduced pressure. This resulted in $140 \mathrm{mg}$ (82\%) of 2-[(E)-2-(4-aminophenyl)ethenyl]- $N$-(5-tert-butyl-1H-pyrazol-3-yl)-6-(4methylpiperazin-1-yl)pyrimidin-4-amine as a crude yellow solid. LCMS (ESI): [M+H]+: 433.4. 
<smiles>C=CC(=O)Nc1ccc(/C=C/c2nc(Nc3cc(C(C)(C)C)[nH]n3)cc(N3CCN(C)CC3)n2)cc1</smiles>

$N$-[4-[(E)-2-[4-[(5-tert-Butyl-1H-pyrazol-3-yl)amino]-6-(4-methylpiperazin-1-yl)pyrimidin-2-

yl]ethenyl]phenyl]prop-2-enamide (Compound 8): To a mixture of 2-[(E)-2-(4-aminophenyl)ethenyl]- $N$ (5-tert-butyl-1H-pyrazol-3-yl)-6-(4-methylpiperazin-1-yl)pyrimidin-4-amine (120 mg, 0.277 mmol, 1 equiv) in DCM $(10 \mathrm{~mL})$ were added triethylamine $(84 \mathrm{mg}, 0.832 \mathrm{mmol}, 3$ equiv) and acryloyl chloride (25 $\mathrm{mg}$, $0.277 \mathrm{mmol}, 1$ equiv) dropwise at $0{ }^{\circ} \mathrm{C}$. The resulting mixture was stirred at $0{ }^{\circ} \mathrm{C}$ for 2 hours. The reaction was quenched by the addition of $\mathrm{MeOH}$. The resulting mixture was concentrated under vacuum. The crude product was purified by Prep-HPLC with the following conditions: Column: XBridge Shield RP18 OBD Column, 30*150mm,5um ; Mobile Phase A:Water(10mmoL/L $\left.\mathrm{NH}_{4} \mathrm{HCO}_{3}\right)$, Mobile Phase B:ACN; Flow rate:60 mL/min; Gradient:37 B to 60 B in $7 \mathrm{~min}$; Detector, $254 \mathrm{~nm}$; This resulted in $18.7 \mathrm{mg}$ (14\%) of $\mathrm{N}$-[4[(E)-2-[4-[(5-tert-butyl-1H-pyrazol-3-yl)amino]-6-(4-methylpiperazin-1-yl)pyrimidin-2-

yl]ethenyl]phenyl]prop-2-enamide as a light yellow solid. LCMS (ESI): [M+H] $]^{+}$487.2. ${ }^{1} \mathrm{H} \mathrm{NMR}(300 \mathrm{MHz}$, $\left.\mathrm{CD}_{3} \mathrm{OD}\right) \delta 7.85-7.75(\mathrm{~m}, 1 \mathrm{H}), 7.72(\mathrm{~d}, J=8.7 \mathrm{~Hz}, 2 \mathrm{H}), 7.60(\mathrm{~d}, J=8.7 \mathrm{~Hz}, 2 \mathrm{H}), 6.98-6.90(\mathrm{~m}, 1 \mathrm{H}), 6.55(\mathrm{~m}$, $1 \mathrm{H}), 6.33(\mathrm{~m}, 1 \mathrm{H}), 6.31(\mathrm{~s}, 1 \mathrm{H}), 6.08(\mathrm{~s}, 1 \mathrm{H}), 5.84-5.76(\mathrm{~m}, 1 \mathrm{H}), 3.69(\mathrm{~m}, 4 \mathrm{H}), 2.56(\mathrm{~m}, 4 \mathrm{H}), 2.37(\mathrm{~s}, 3 \mathrm{H})$, $1.38(\mathrm{~s}, 9 \mathrm{H})$.

\section{Compound 9:}
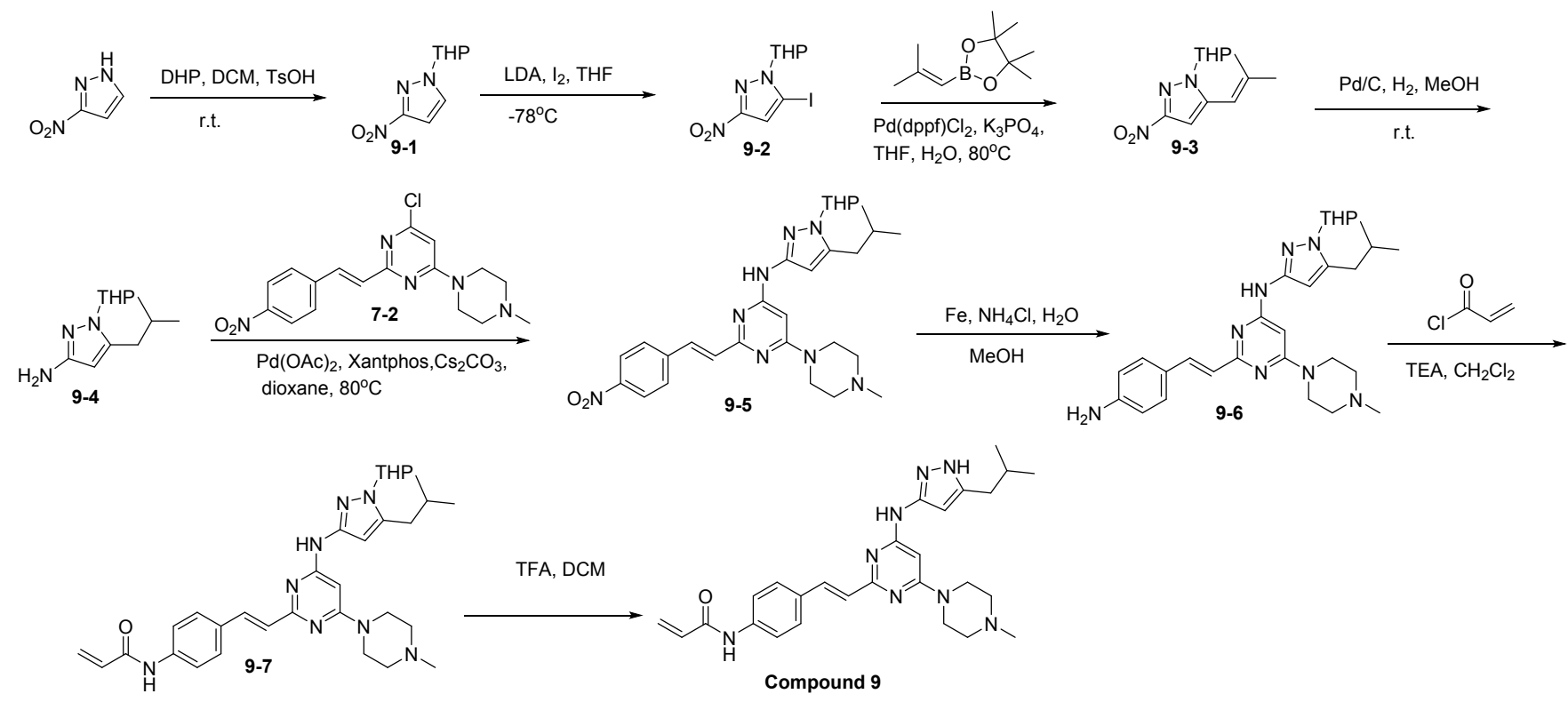


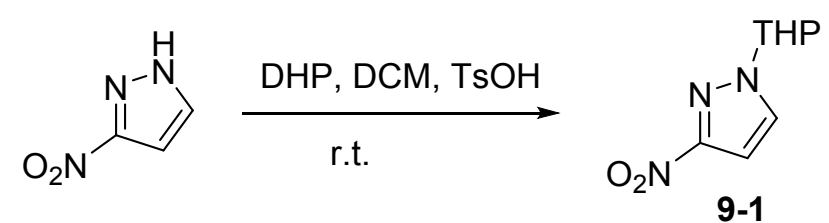

3-Nitro-1-(oxan-2-yl)pyrazole (9-1): To a mixture of 3-nitro-1H-pyrazole (16.0 g, $141.5 \mathrm{mmol}$ ) in DCM (35 $\mathrm{mL})$ was added $\mathrm{DHP}(17.9 \mathrm{~g}, 212.25 \mathrm{mmol})$ and $\mathrm{TsOH}(3.7 \mathrm{~g}, 21.23 \mathrm{mmol})$ at $0{ }^{\circ} \mathrm{C}$. The resulting mixture was stirred overnight at $25{ }^{\circ} \mathrm{C}$. After the reaction was completed the crude product was concentrated under reduced pressure. The residue was purified by silica gel column chromatography eluting with $\mathrm{PE} /$ EtOAc $(5 / 1, \mathrm{v} / \mathrm{v})$ to afford 3-nitro-1-(oxan-2-yl)pyrazole $(18.16 \mathrm{~g}, 65 \%)$ as an off-white oil.<smiles>O=[N+]([O-])c1cc(I)n([TlH])n1</smiles>

5-Iodo-3-nitro-1-(oxan-2-yl)pyrazole (9-2): To a mixture of 3-nitro-1-(oxan-2-yl)pyrazole (16.0 g, 81.14 $\mathrm{mmol})$ in THF $(50 \mathrm{~mL})$ was added LDA $(10.4 \mathrm{~g}, 97.37 \mathrm{mmol})$ dropwise at $-78^{\circ} \mathrm{C}$ under nitrogen atmosphere. The mixture was stirred at $-78{ }^{\circ} \mathrm{C}$ for 30 minutes under nitrogen atmosphere. Then iodine $(24.7 \mathrm{~g}, 97.37$ mmol) was added slowly at $-78{ }^{\circ} \mathrm{C}$ under a nitrogen atmosphere. The resulting mixture was stirred for 3 hours at $-78{ }^{\circ} \mathrm{C}$ under a nitrogen atmosphere. After the reaction was completed, the reaction was quenched with sat. $\mathrm{NH}_{4} \mathrm{Cl}$. The mixture was diluted with $\mathrm{H}_{2} \mathrm{O}$ and extracted with ethyl acetate. The combined organic layer was washed with brine, dried over $\mathrm{Na}_{2} \mathrm{SO}_{4}$ and filtered. The filtrate was evaporated under vacuum. The residue was purified by silica gel column chromatography eluting with PE/EtOAc ether (8/1) to afford 5-iodo-3-nitro-1-(oxan-2-yl)pyrazole (12.2g, 47\%) as a yellow solid. ${ }^{3}$
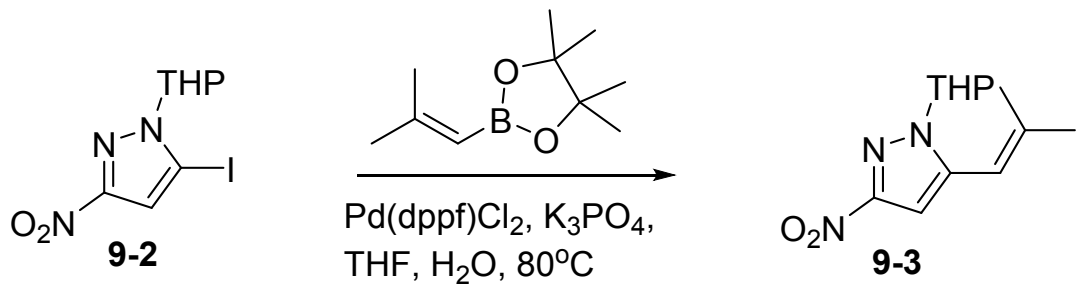

\footnotetext{
${ }^{3}$ For precedence see: Twigg, D. G.; et al. Partially Saturated Bicyclic Heteroaromatics as an $\mathrm{sp}^{3}$ - Enriched Fragment Collection. Angew. Chem. Int. Ed. 2016, 55, 12479-12483.
} 
5-(2-Methylprop-1-en-1-yl)-3-nitro-1-(oxan-2-yl)pyrazole (9-3): To a mixture of 5-iodo-3-nitro-1-(oxan-2yl)pyrazole $(1.0 \mathrm{~g}, 3.10 \mathrm{mmol})$ in THF $(10 \mathrm{~mL}), \mathrm{H}_{2} \mathrm{O}(1 \mathrm{~mL})$ was added 4,4,5,5-tetramethyl-2-(2-methylprop1-en-1-yl)-1,3,2-dioxaborolane (2.8 g, $15.5 \mathrm{mmol}), \mathrm{K}_{3} \mathrm{PO}_{4}(2.0 \mathrm{~g}, 9.29 \mathrm{mmol})$ and $\mathrm{Pd}(\mathrm{dppf}) \mathrm{Cl}_{2}(0.2 \mathrm{~g}, 0.31$ $\mathrm{mmol}$ ) in portions at $25^{\circ} \mathrm{C}$ under nitrogen atmosphere. The resulting mixture was stirred for overnight at $80^{\circ} \mathrm{C}$ under nitrogen atmosphere. After the reaction was completed, the mixture was evaporated under vacuum. The residue was purified by silica gel column chromatography eluting with PE/EtOAc $(8 / 1, v / v)$ to afford 5-(2-methylprop-1-en-1-yl)-3-nitro-1-(oxan-2-yl)pyrazole (750 mg, 96\%) as a yellow solid.

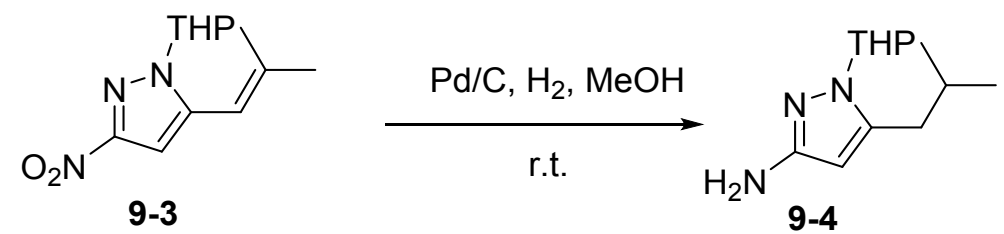

5-(2-Methylpropyl)-1-(oxan-2-yl)pyrazol-3-amine (9-4): To a mixture of 5-(2-methylprop-1-en-1-yl)-3nitro-1-(oxan-2-yl)pyrazole $(750 \mathrm{mg}, 2.99 \mathrm{mmol}$ ) in $\mathrm{MeOH}(5 \mathrm{~mL})$ was added $\mathrm{Pd} / \mathrm{C}(225 \mathrm{mg}, 2.11 \mathrm{mmol})$. The mixture was stirred at room temperature overnight under $\mathrm{H}_{2}$ atmosphere. After the reaction was completed, the resulting mixture was filtered, the filter cake was washed with $\mathrm{MeOH}$. The filtrate was concentrated under reduced pressure to afford 5-(2-methylpropyl)-1-(oxan-2-yl)pyrazol-3-amine (790 mg, crude) as a brown oil. LCMS (ESI, $\mathrm{m} / \mathrm{z}):[\mathrm{M}+\mathrm{H}]^{+}: 224.2$.
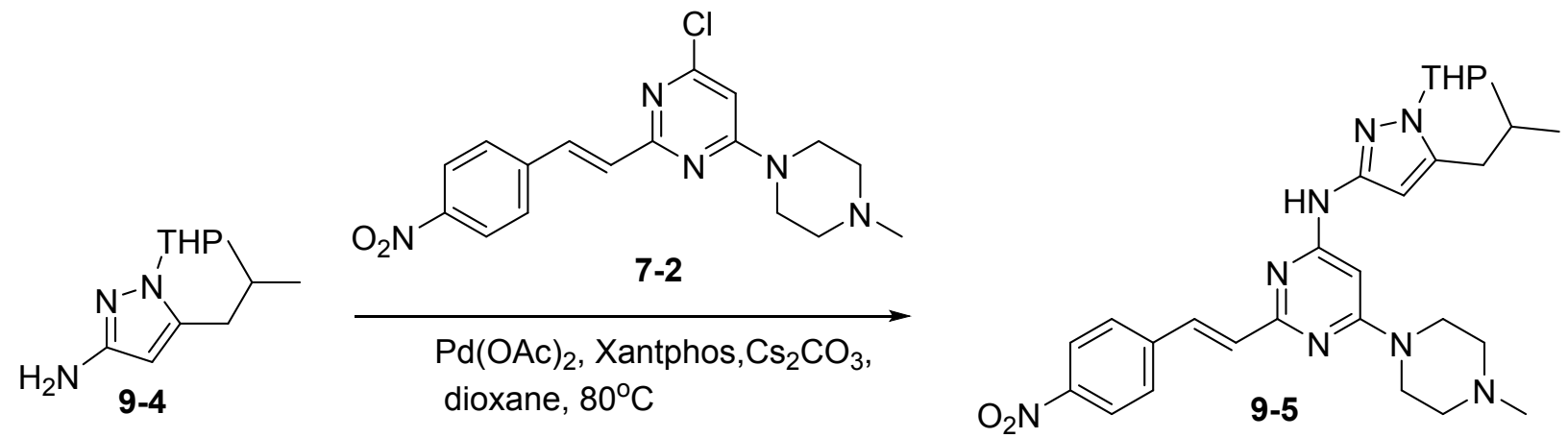

6-(4-Methylpiperazin-1-yl)-N-[5-(2-methylpropyl)-1-(oxan-2-yl)pyrazol-3-yl]-2-[(E)-2-(4-

nitrophenyl)ethenyl]pyrimidin-4-amine (9-5): To a mixture of 5-(2-methylpropyl)-1-(oxan-2-yl)pyrazol3-amine $(480 \mathrm{mg}, 2.15 \mathrm{mmol})$ in dioxane $(10 \mathrm{~mL})$ was added 4-chloro-6-(4-methylpiperazin-1-yl)-2-[(E)-2(4-nitrophenyl)ethenyl]pyrimidine (773.4 mg, $2.149 \mathrm{mmol}), \mathrm{Pd}(\mathrm{AcO})_{2}(48.3 \mathrm{mg}, 0.22 \mathrm{mmol}$ ) and Xantphos (254 mg, $0.44 \mathrm{mmol}$ ) in portions at $25^{\circ} \mathrm{C}$ under nitrogen atmosphere. The resulting mixture was stirred for overnight at $80{ }^{\circ} \mathrm{C}$ under nitrogen atmosphere. After the reaction was completed, the mixture was evaporated under vacuum. The residue was purified by silica gel column chromatography with DCM/MeOH (10/1, v/v) to afford 6-(4-methylpiperazin-1-yl)-N-[5-(2-methylpropyl)-1-(oxan-2-yl)pyrazol3-yl]-2-[(E)-2-(4-nitrophenyl)ethenyl]pyrimidin-4-amine (460 mg, 39\%) as a yellow solid. LCMS (ESI, m/z): $[\mathrm{M}+\mathrm{H}]^{+}: 547.3$. 
<smiles>CC(P)Cc1cc(Nc2cc(N3CCN(C)CC3)nc(/C=C/c3ccc(N)cc3)n2)nn1[Tl]</smiles>

$\boldsymbol{N}$-[(3-Aminophenyl)methyl]-6-[imidazo[1,2-a]pyridin-3-yl]pyrazine-2-carboxamide (9-6): To a mixture of 6-(4-methylpiperazin-1-yl)- $N$-[5-(2-methylpropyl)-1-(oxan-2-yl)pyrazol-3-yl]-2-[(E)-2-(4nitrophenyl)ethenyl]pyrimidin-4-amine (460 mg, $0.84 \mathrm{mmol})$ in $\mathrm{MeOH}(10 \mathrm{~mL})$ was added $\mathrm{H}_{2} \mathrm{O}(1 \mathrm{~mL})$, $\mathrm{NH}_{4} \mathrm{Cl}\left(234.9 \mathrm{mg}, 4.20 \mathrm{mmol}\right.$ ) and iron $(234.9 \mathrm{mg}, 4.20 \mathrm{mmol})$. The mixture was stirred at $80^{\circ} \mathrm{C}$ for 2 hours. After the reaction was completed, the mixture was evaporated under vacuum. The residue was purified by flash column chromatography with $\mathrm{DCM} / \mathrm{MeOH}(1 / 1, \mathrm{v} / \mathrm{v})$ to afford 2-[(E)-2-(4-aminophenyl)ethenyl]6-(4-methylpiperazin-1-yl)- $N$-[5-(2-methylpropyl)-1-(oxan-2-yl)pyrazol-3-yl]pyrimidin-4-amine (230 mg, $52 \%)$ as a yellow solid. LCMS (ESI, $\mathrm{m} / \mathrm{z}):[\mathrm{M}+\mathrm{H}]^{+}: 517.3$.<smiles>CC(P)Cc1cc(Nc2cc(N3CCN(C)CC3)nc(/C=C/c3ccc(N)cc3)n2)nn1[Tl]</smiles><smiles>C=CC(=O)Nc1ccc(/C=C/c2nc(Nc3cc(CC(C)C)n([Tl])n3)cc(N3CCN(C)CC3)n2)cc1</smiles>

$N$-[4-[(E)-2-[4-(4-methylpiperazin-1-yl)-6-[[5-(2-methylpropyl)-1-(oxan-2-yl)pyrazol-3-

yl]amino]pyrimidin-2-yl]ethenyl]phenyl]prop-2-enamide (9-7): To a stirred mixture of 2-[(E)-2-(4aminophenyl)ethenyl]-6-(4-methylpiperazin-1-yl)- $N$-[5-(2-methylpropyl)-1-(oxan-2-yl)pyrazol-3-

yl]pyrimidin-4-amine (205 mg, 0.397 mmol, 1 equiv) in DCM were added triethylamine (120.4 mg, 1.190 mmol, 3 equiv) and acryloyl chloride (43.1 $\mathrm{mg}, 0.476 \mathrm{mmol}, 1.2$ equiv) dropwise at room temperature. The final reaction mixture was irradiated with microwave radiation for $30 \mathrm{~min}$ at room temperature. The mixture was evaporated under vacuum. The residue was purified by reverse phase column chromatography, eluting with ACN/H2O (1/1) to afford N-[4-[(E)-2-[4-(4-methylpiperazin-1-yl)-6-[[5-(2methylpropyl)-1-(oxan-2-yl)pyrazol-3-yl]amino]pyrimidin-2-yl]ethenyl]phenyl]prop-2-enamide (200mg, $88 \%$ ) as a yellow solid. LCMS (ESI, $\mathrm{m} / \mathrm{z}$ ): $[\mathrm{M}+\mathrm{H}]^{+}: 571.3$. 
<smiles>C=CC(=O)Nc1ccc(/C=C/c2nc(Nc3cc(CC(C)P)n([Tl])n3)cc(N3CCN(C)CC3)n2)cc1</smiles>

$N$-[4-[(E)-2-[4-(4-methylpiperazin-1-yl)-6-[[5-(2-methylpropyl)-1H-pyrazol-3-yl]amino]pyrimidin-2-

yl]ethenyl]phenyl]prop-2-enamide (Compound 9): To a mixture of $N$-[4-[(E)-2-[4-(4-methylpiperazin-1yl)-6-[[5-(2-methylpropyl)-1-(oxan-2-yl)pyrazol-3-yl]amino]pyrimidin-2-yl]ethenyl]phenyl]prop-2-

enamide $(250 \mathrm{mg}, 0.44 \mathrm{mmol})$ in DCM $(6 \mathrm{~mL})$ was added TFA $(3 \mathrm{~mL})$. The mixture was stirred at room temperature for 2 hours. The resulting mixture was concentrated under vacuum. The residue was purified by Prep-HPLC with the following conditions: Column: Xselect CSH OBD Column 30*150mm 5um, n; Mobile Phase A:Water(0.1\%FA), Mobile Phase B:ACN; Flow rate:60 mL/min; Gradient:12 B to 25 B in 8 min; 254/220 nm; RT1:7.53; to afford $N$-[4-[(E)-2-[4-(4-methylpiperazin-1-yl)-6-[[5-(2-methylpropyl)-1Hpyrazol-3-yl]amino]pyrimidin-2-yl]ethenyl]phenyl]prop-2-enamide(2.4 $\mathrm{mg}, 1 \%)$ as a yellow solid. LCMS (ESI, m/z): [M+H] $]^{+}: 487.3 .{ }^{1} \mathrm{H}$ NMR (300 MHz, DMSO-d $\left.d_{6}\right): \delta 10.40(\mathrm{~s}, 1 \mathrm{H}), 9.21(\mathrm{~s}, 1 \mathrm{H}), 8.17(\mathrm{~s}, 1 \mathrm{H}), 7.74-$ $7.60(\mathrm{~m}, 5 \mathrm{H}), 6.85(\mathrm{~d}, J=15.9 \mathrm{~Hz}, 1 \mathrm{H}), 6.64(\mathrm{~s}, 1 \mathrm{H}), 6.51-6.42(\mathrm{~m}, 1 \mathrm{H}), 6.31(\mathrm{~d}, J=2.1 \mathrm{~Hz}, 1 \mathrm{H}), 5.99(\mathrm{~s}, 1 \mathrm{H})$, $55.81-5.77(\mathrm{~s}, 4 \mathrm{H}), 2.44-2.42(\mathrm{~m}, 6 \mathrm{H}), 2.27-2.24(\mathrm{~m}, 3 \mathrm{H}), 1.95-1.84(\mathrm{~m}, 1 \mathrm{H}), 0.92-0.84(\mathrm{~m}, 6 \mathrm{H})$.

\section{Compound 10:}
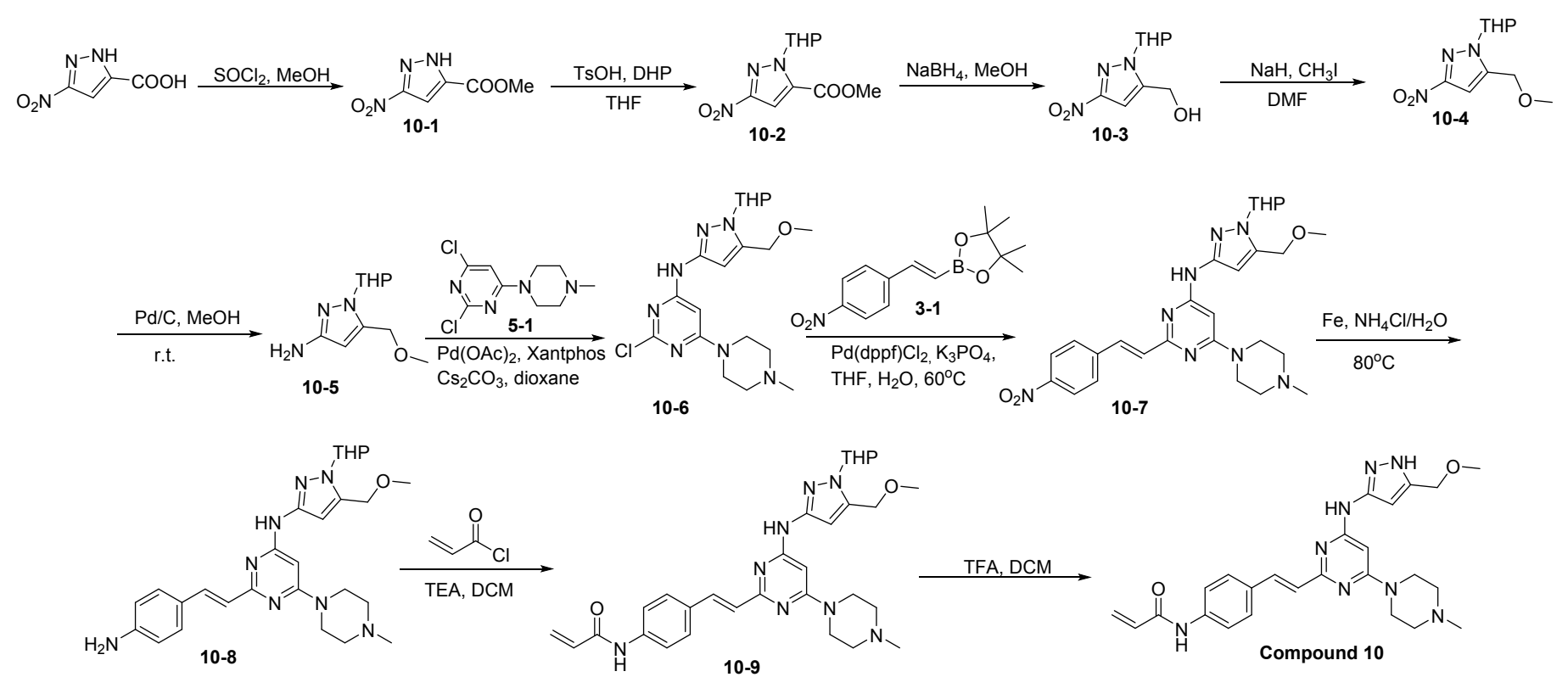


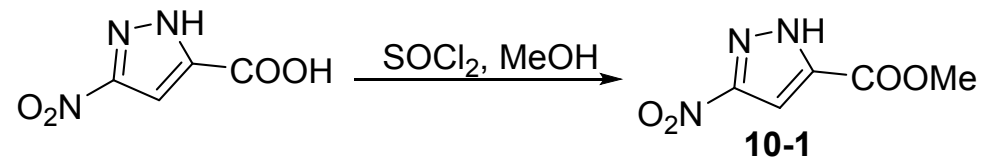

Methyl 5-nitro-2H-pyrazole-3-carboxylate (10-1): To a mixture of 5-nitro-2H-pyrazole-3-carboxylic acid (2 g, $12.732 \mathrm{mmol}, 1$ equiv) in $\mathrm{MeOH}(50 \mathrm{~mL})$ was added $\mathrm{SOCl}_{2}(16 \mathrm{~mL})$. The resulting mixture was stirred at room temperature overnight. The resulting mixture was concentrated under vacuum. This resulted in $2.5 \mathrm{~g}$ (crude) methyl 5-nitro-2H-pyrazole-3-carboxylate as a white solid. LCMS (ESI): [M+H]+: 172.0.<smiles>COC(=O)c1cc([N+](=O)[O-])n[nH]1</smiles>

Methyl 5-nitro-2-(oxan-2-yl)pyrazole-3-carboxylate (10-2): To a mixture of methyl 5-nitro-2H-pyrazole3-carboxylate ( $5 \mathrm{~g}, 29.221 \mathrm{mmol}, 1$ equiv) in THF (60 mL) were added DHP (4.92 g, $58.441 \mathrm{mmol}, 2$ equiv) and $\mathrm{TsOH}(2.01 \mathrm{~g}, 11.688 \mathrm{mmol}, 0.4$ equiv). The resulting mixture was stirred at room temperature overnight. The resulting mixture was concentrated under vacuum. The residue was applied onto a silica gel column, eluting with ethyl acetate/petroleum ether (1/1). This resulted in $3.9 \mathrm{~g}$ (52\%) of methyl 5nitro-2-(oxan-2-yl)pyrazole-3-carboxylate as a white solid. LCMS (ESI): [M+H] ${ }^{+}: 256.1$.<smiles>COC(=O)c1cc([N+](=O)[O-])nn1[InH]c1cc([N+](=O)[O-])nn1[InH]</smiles>

[5-Nitro-2-(oxan-2-yl)pyrazol-3-yl]methanol (10-3): To a mixture of methyl 5-nitro-2-(oxan-2-yl)pyrazole3-carboxylate (3.9 g, $15.28 \mathrm{mmol}, 1$ equiv) in $\mathrm{MeOH}(100 \mathrm{~mL})$ was added $\mathrm{NaBH}_{4}(1.16 \mathrm{~g}, 30.713 \mathrm{mmol}$, 2.01 equiv). The resulting mixture was stirred at room temperature overnight. The resulting mixture was quenched with water and extracted with $3 \times 10 \mathrm{~mL}$ of ethyl acetate. The combined organic layers were dried over anhydrous sodium sulfate, filtered and concentrated under vacuum. This resulted in $1.9 \mathrm{~g} \mathrm{(55 \% )}$ of [5-nitro-2-(oxan-2-yl)pyrazol-3-yl]methanol as a white solid. LCMS (ESI): [M+H]+: 228.1.<smiles>COCc1cc([N+](=O)[O-])nn1[In]CC(C)N</smiles> 
5-(Methoxymethyl)-3-nitro-1-(oxan-2-yl)pyrazole (10-4): To a mixture of [5-nitro-2-(oxan-2-yl)pyrazol-3yl]methanol (1.9 g, $8.362 \mathrm{mmol}, 1$ equiv) in DMF (20 mL) was added $\mathrm{NaH}(0.4 \mathrm{~g}, 16.724 \mathrm{mmol}, 2$ equiv). The resulting solution was stirred at $0^{\circ} \mathrm{C}$ for 30 minutes. Then iodomethane $(1.78 \mathrm{~g}, 12.543 \mathrm{mmol}, 1.5$ equiv) was added with stirring. The resulting mixture was stirred at room temperature overnight. The resulting mixture was quenched with water/ice and extracted with $3 \times 10 \mathrm{~mL}$ of ethyl acetate. The combined organic layers were dried over anhydrous sodium sulfate, filtered and concentrated under vacuum. The residue was applied onto a silica gel column eluting with ethyl acetate/petroleum ether (1/1). This resulted in $1.5 \mathrm{~g}$ (74\%) of 5-(methoxymethyl)-3-nitro-1-(oxan-2-yl)pyrazole as a white solid. LCMS (ESI): $[\mathrm{M}+\mathrm{H}]^{+}: 242.1$.

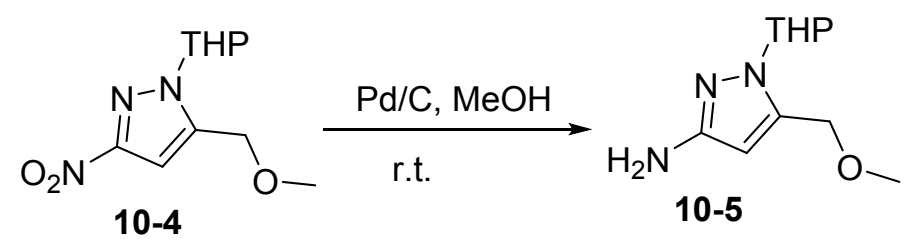

5-(Methoxymethyl)-1-(oxan-2-yl)pyrazol-3-amine (10-5): To a mixture of 5-(methoxymethyl)-3-nitro-1(oxan-2-yl)pyrazole (1.5 g, $6.218 \mathrm{mmol}, 1$ equiv) in $\mathrm{MeOH}(20 \mathrm{~mL})$. The resulting mixture was stirred at room temperature under hydrogen atmosphere for 1 hour. The solids were filtered out. The resulting mixture was concentrated under vacuum. This resulted in $1.3 \mathrm{~g}$ (crude) 5-(methoxymethyl)-1-(oxan-2yl)pyrazol-3-amine as a white solid. LCMS (ESI) $[\mathrm{M}+\mathrm{H}]^{+}: 212.1$.

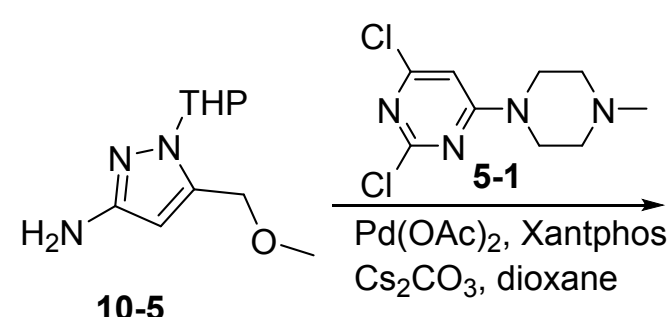

10-5

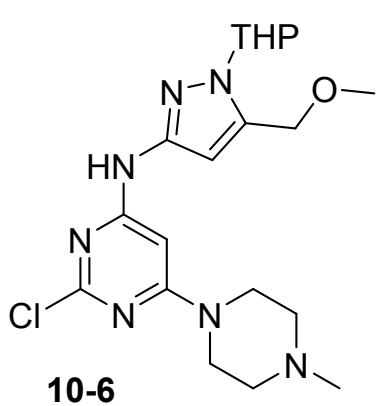

10-6

\section{2-Chloro-N-[5-(methoxymethyl)-1-(oxan-2-yl)pyrazol-3-yl]-6-(4-methylpiperazin-1-yl)pyrimidin-4-}

amine (10-6): To a mixture of 5-(methoxymethyl)-1-(oxan-2-yl)pyrazol-3-amine (850 mg, $4.023 \mathrm{mmol}, 1$ equiv) and 2,4-dichloro-6-(4-methylpiperazin-1-yl)pyrimidine (1.094 g, $4.426 \mathrm{mmol}, 1.1$ equiv) in dioxane $\left(10 \mathrm{~mL}\right.$ ) were added XantPhos (466 mg, $0.805 \mathrm{mmol}, 0.2$ equiv), $\mathrm{Pd}(\mathrm{OAc})_{2}$ (91 mg, $0.402 \mathrm{mmol}, 0.1$ equiv) and $\mathrm{Cs}_{2} \mathrm{CO}_{3}$ ( $3.933 \mathrm{~g}, 12.07 \mathrm{mmol}, 3$ equiv). The resulting mixture was stirred at $50{ }^{\circ} \mathrm{C}$ overnight. The resulting mixture was concentrated under vacuum. The residue was applied onto a silica gel column eluting with dichloromethane/methanol (10/1). This resulted in $800 \mathrm{mg}$ (47\%) 2-chloro- $N$-[5(methoxymethyl)-1-(oxan-2-yl)pyrazol-3-yl]-6-(4-methylpiperazin-1-yl)pyrimidin-4-amine as a yellow solid. $\mathrm{LCMS}(\mathrm{ESI})[\mathrm{M}+\mathrm{H}]^{+}:$: 422.2. Structures were assigned by $1 \mathrm{H} N \mathrm{NM}$ analysis after de-chlorination. 
<smiles>COCc1cc(Nc2cc(N3CCN(C)CC3)nc(Cl)n2)nc(Nc2cc(N3CCN(C)CC3)nc(/C=C/c3ccc([N+](=O)[O-])cc3)n2)c1</smiles>

N-[5-(Methoxymethyl)-1-(oxan-2-yl)pyrazol-3-yl]-6-(4-methylpiperazin-1-yl)-2-[(E)-2-(4nitrophenyl)ethenyl]pyrimidin-4-amine (10-7): To a mixture of 2-chloro- $N$-[5-(methoxymethyl)-1-(oxan2-yl)pyrazol-3-yl]-6-(4-methylpiperazin-1-yl)pyrimidin-4-amine (180 mg, $0.427 \mathrm{mmol}, 1$ equiv) and 4,4,5,5-tetramethyl-2-[(E)-2-(4-nitrophenyl)ethenyl]-1,3,2-dioxaborolane (65 $\mathrm{mg}, 0.237 \mathrm{mmol}, 5$ equiv) in THF $(5 \mathrm{~mL})$ were added $\mathrm{H}_{2} \mathrm{O}(0.5 \mathrm{~mL}), \mathrm{Pd}(\mathrm{dppf}) \mathrm{Cl}_{2}\left(32 \mathrm{mg}, 0.043 \mathrm{mmol}, 0.1\right.$ equiv) and $\mathrm{K}_{3} \mathrm{PO}_{4}(272 \mathrm{mg}, 1.28$ mmol, 3 equiv). The resulting mixture was stirred at $80{ }^{\circ} \mathrm{C}$ overnight. The resulting mixture was concentrated under vacuum. The residue was applied onto a silica gel column eluting with dichloromethane/methanol (10/1). This resulted in $170 \mathrm{mg}$ (75\%) N-[5-(methoxymethyl)-1-(oxan-2yl)pyrazol-3-yl]-6-(4-methylpiperazin-1-yl)-2-[(E)-2-(4-nitrophenyl)ethenyl]pyrimidin-4-amine as a yellow solid. LCMS (ESI) $[\mathrm{M}+\mathrm{H}]^{+}$: 535.3 .<smiles>COCc1cc(Nc2cc(N3CCN(C)CC3)nc(/C=C/c3ccc([N+](=O)[O-])cc3)n2)nn1[TlH]</smiles><smiles>COCc1cc(Nc2cc(N3CCN(C)CC3)nc(/C=C/c3ccc(N)cc3)n2)nn1[In]</smiles>

\section{2-[(E)-2-(4-Aminophenyl)ethenyl]- $N$-[5-(methoxymethyl)-1-(oxan-2-yl)pyrazol-3-yl]-6-(4-}

methylpiperazin-1-yl)pyrimidin-4-amine (10-8): To a mixture of $\mathrm{N}$-[5-(methoxymethyl)-1-(oxan-2yl)pyrazol-3-yl]-6-(4-methylpiperazin-1-yl)-2-[(E)-2-(4-nitrophenyl)ethenyl]pyrimidin-4-amine (150 mg, $0.281 \mathrm{mmol}, 1$ equiv) in $\mathrm{MeOH}(10 \mathrm{~mL}$ ) were added iron $(157 \mathrm{mg}, 2.806 \mathrm{mmol}, 10$ equiv) and saturated aqueous $\mathrm{NH}_{4} \mathrm{Cl}(1 \mathrm{~mL})$. The resulting mixture was stirred at $80^{\circ} \mathrm{C}$ for 1 hour. The resulting mixture was concentrated under vacuum. The residue was applied onto a silica gel column eluting with dichloromethane/methanol (10/1). This resulted in 100 mg (71\%) 2-[(E)-2-(4-aminophenyl)ethenyl]- $N$-[5(methoxymethyl)-1-(oxan-2-yl)pyrazol-3-yl]-6-(4-methylpiperazin-1-yl)pyrimidin-4-amine as a yellow solid. LCMS (ESI) [M+H]+: 505.3. 
<smiles>C=CC(=O)Nc1ccc(/C=C/c2nc(Nc3cc(COC)n([Tl])n3)cc(N3CCN(C)CC3)n2)cc1</smiles>

$N$-[4-[(E)-2-(4-[[5-(Methoxymethyl)-1-(oxan-2-yl)pyrazol-3-yl]amino]-6-(4-methylpiperazin-1yl)pyrimidin-2-yl)ethenyl]phenyl]prop-2-enamide (10-9): To a mixture of 2-[(E)-2-(4aminophenyl)ethenyl]- $N$-[5-(methoxymethyl)-1-(oxan-2-yl)pyrazol-3-yl]-6-(4-methylpiperazin-1yl)pyrimidin-4-amine (80 mg, $0.159 \mathrm{mmol}, 1$ equiv) in DCM (10 mL) were added triethylamine (49 $\mathrm{mg}$, $0.476 \mathrm{mmol}, 3$ equiv) and acryloyl chloride $\left(15 \mathrm{mg}, 0.159 \mathrm{mmol}, 1\right.$ equiv) at $0{ }^{\circ} \mathrm{C}$. The resulting mixture was stirred at $0{ }^{\circ} \mathrm{C}$ for 1 hour. The resulting mixture was concentrated under vacuum. The residue was applied onto a silica gel column eluting with dichloromethane/methanol (10/1). This resulted in $30 \mathrm{mg}$ $(34 \%)$ $\mathrm{N}$-[4-[(E)-2-(4-[[5-(methoxymethyl)-1-(oxan-2-yl)pyrazol-3-yl]amino]-6-(4-methylpiperazin-1yl)pyrimidin-2-yl)ethenyl]phenyl]prop-2-enamide as a yellow solid. LCMS (ESI) [M+H]+: 559.3 .<smiles>C=CC(=O)Nc1ccc(/C=C/c2nc(NC(=O)C=C)nc(Nc3ccc(/C=C/c4cc(COC)n([Tl])n4)cc3N3CCN(C)CC3)n2)cc1</smiles>

$N$-[4-[(E)-2-(4-[[5-(Methoxymethyl)-1H-pyrazol-3-yl]amino]-6-(4-methylpiperazin-1-yl)pyrimidin-2yl)ethenyl]phenyl]prop-2-enamide (Compound 10): To a mixture of $N$-[4-[(E)-2-(4-[[5-(methoxymethyl)1-(oxan-2-yl)pyrazol-3-yl]amino]-6-(4-methylpiperazin-1-yl)pyrimidin-2-yl)ethenyl]phenyl]prop-2enamide (30 mg, $0.054 \mathrm{mmol}, 1$ equiv) in DCM (5 mL) were added TFA (1 mL). The resulting mixture was stirred at $0{ }^{\circ} \mathrm{C}$ for 1 hour. The resulting mixture was concentrated under vacuum. The crude product was purified by Prep-HPLC with the following conditions: Column: XBridge Prep OBD C18 Column, 30×150mm 5um; Mobile Phase A: Water (10mmol/ $\left.\mathrm{L} \mathrm{NH}_{4} \mathrm{HCO}_{3}\right)$, Mobile Phase B: ACN; Flow rate: $60 \mathrm{~mL} / \mathrm{min}$; Gradient: 26 B to 56 B in $7 \mathrm{~min}$; Detector, $254 \mathrm{~nm}$; RT1: 5.93. This resulted in $1.6 \mathrm{mg}(6 \%)$ of $N$-[4-[(E)-2-(4-[[5(methoxymethyl)-1H-pyrazol-3-yl]amino]-6-(4-methylpiperazin-1-yl)pyrimidin-2-yl)ethenyl]phenyl]prop2-enamide as a yellow solid. LCMS (ESI) $[\mathrm{M}+\mathrm{H}]^{+}$: 475.4. ${ }^{1} \mathrm{H} \mathrm{NMR}\left(300 \mathrm{MHz}, \mathrm{CD}_{3} \mathrm{OD}\right) \delta 7.93-7.56(\mathrm{~m}, 6 \mathrm{H})$, $7.51-6.75(\mathrm{~m}, 2 \mathrm{H}), 6.62-6.14(\mathrm{~m}, 2 \mathrm{H}), 5.91-5.64(\mathrm{~m}, 1 \mathrm{H}), 4.47(\mathrm{~s}, 2 \mathrm{H}), 3.78-3.59(\mathrm{~m}, 4 \mathrm{H}), 3.39(\mathrm{~s}, 3 \mathrm{H})$, $2.69-2.44(\mathrm{~m}, 4 \mathrm{H}), 2.35(\mathrm{~s}, 3 \mathrm{H})$. 


\section{Compound 11:}
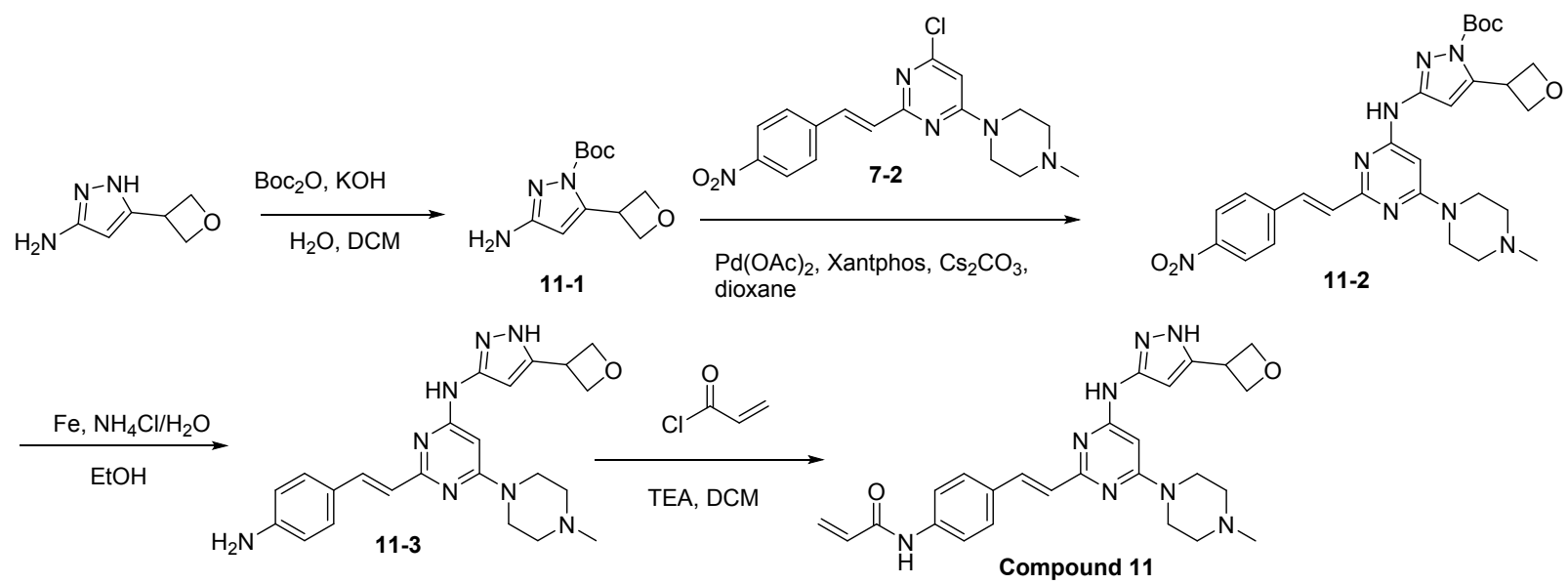



tert-Butyl 3-amino-5-(oxetan-3-yl)pyrazole-1-carboxylate (11-1): To a mixture of 5-(oxetan-3-yl)- $1 \mathrm{H}$ pyrazol-3-amine (500 mg, $3.593 \mathrm{mmol}, 1$ equiv) in DCM $(50 \mathrm{~mL})$ were added $\mathrm{KOH}(1.61 \mathrm{~g}, 28.744 \mathrm{mmol}$, 8 equiv) in $\mathrm{H}_{2} \mathrm{O}\left(7.2 \mathrm{~mL}\right.$ ) and $\mathrm{Boc}_{2} \mathrm{O}\left(941 \mathrm{mg}, 4.312 \mathrm{mmol}, 1.2\right.$ equiv) at $0{ }^{\circ} \mathrm{C}$. The resulting mixture was stirred at room temperature overnight. The aqueous layer was extracted with $\mathrm{CH}_{2} \mathrm{Cl}_{2}$. The resulting mixture was concentrated under vacuum. The residue was purified by silica gel column chromatography, eluting with PE/EtOAc (1/1) to afford tert-butyl 3-amino-5-(oxetan-3-yl)pyrazole-1-carboxylate (300 mg, $35 \%)$ as a yellow solid. LCMS (ESI): [M+H] $]^{+}: 240.1$.

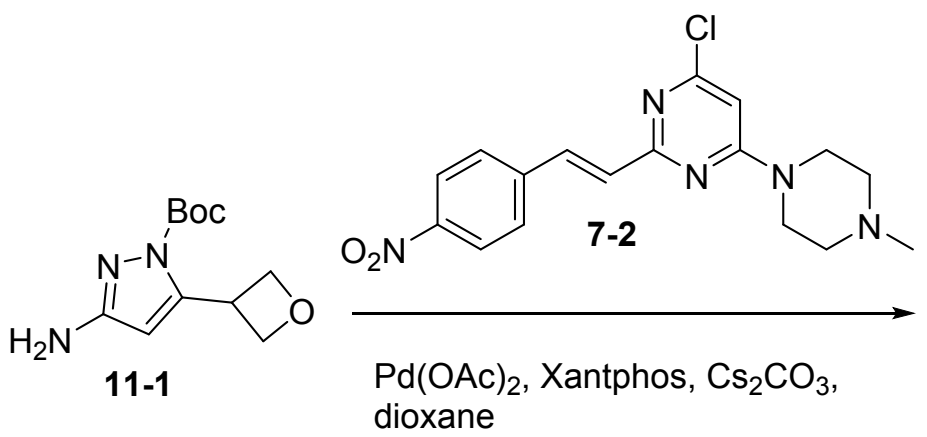<smiles>CN1CCN(c2cc(Nc3cc(C4COC4)n(C(=O)OC(C)(C)C)n3)nc(/C=C/c3ccc([N+](=O)[O-])cc3)n2)CC1</smiles>

tert-Butyl 3-[[6-(4-methylpiperazin-1-yl)-2-[(E)-2-(4-nitrophenyl)ethenyl]pyrimidin-4-yl]amino]-5(oxetan-3-yl)pyrazole-1-carboxylate (11-2): To a mixture of tert-butyl 3-amino-5-(oxetan-3-yl)pyrazole-1- 
carboxylate (300 mg, $1.254 \mathrm{mmol}, 1$ equiv) and 4-chloro-6-(4-methylpiperazin-1-yl)-2-[(E)-2-(4nitrophenyl)ethenyl]pyrimidine (541 mg, $1.505 \mathrm{mmol}, 1.2$ equiv) in dioxane $(5 \mathrm{~mL})$ were added $\mathrm{Cs}_{2} \mathrm{CO}_{3}(1.3$ g, $3.761 \mathrm{mmol}, 3$ equiv), Xantphos ( $145 \mathrm{mg}, 0.251 \mathrm{mmol}, 0.2$ equiv) and $\mathrm{Pd}(\mathrm{OAc})_{2}$ ( $285 \mathrm{mg}, 0.125 \mathrm{mmol}$, 0.1 equiv). The resulting mixture was stirred at $50^{\circ} \mathrm{C}$ overnight under a nitrogen atmosphere. The resulting mixture was concentrated under vacuum. The residue was purified by silica gel column chromatography, eluting with $\mathrm{CH}_{2} \mathrm{Cl}_{2} / \mathrm{MeOH}$ (10/1) to afford tert-butyl 3-[[6-(4-methylpiperazin-1-yl)-2-[(E)-2-(4nitrophenyl)ethenyl]pyrimidin-4-yl]amino]-5-(oxetan-3-yl)pyrazole-1-carboxylate (200 mg, 28\%) as a yellow solid. LCMS (ESI): [M+H]+: 563.5 .

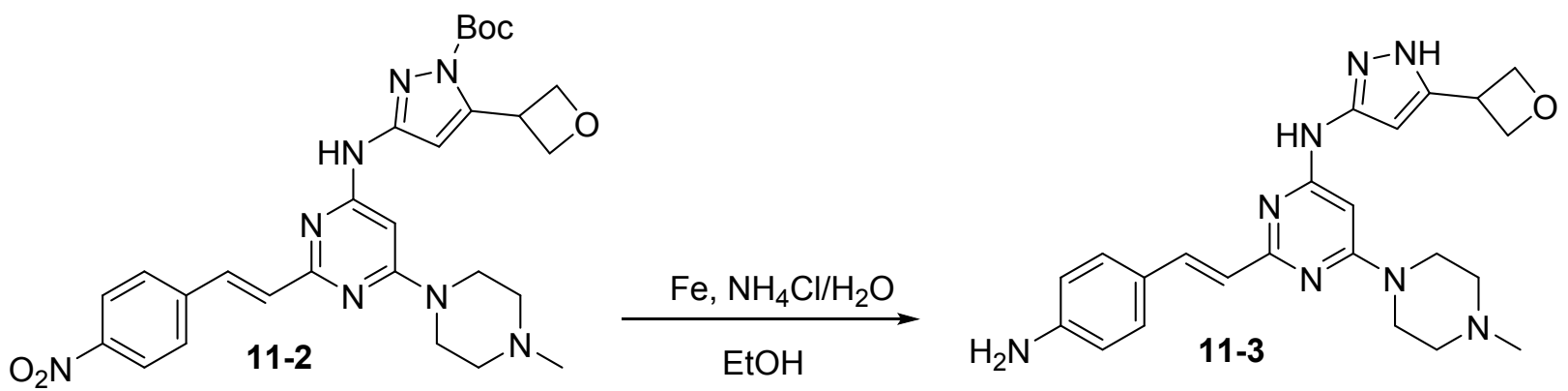

2-[(E)-2-(4-Aminophenyl)ethenyl]-6-(4-methylpiperazin-1-yl)-N-[5-(oxetan-3-yl)-1H-pyrazol-3-

yl]pyrimidin-4-amine (11-3): To a mixture of tert-butyl 3-[[6-(4-methylpiperazin-1-yl)-2-[(E)-2-(4nitrophenyl)ethenyl]pyrimidin-4-yl]amino]-5-(oxetan-3-yl)pyrazole-1-carboxylate (200 mg, $0.355 \mathrm{mmol}, 1$ equiv) and iron (199 mg, $3.555 \mathrm{mmol}, 10$ equiv) in $\mathrm{EtOH}\left(5 \mathrm{~mL}\right.$ ) was added saturated aqueous $\mathrm{NH}_{4} \mathrm{Cl}$ solution $(1 \mathrm{~mL})$. The resulting mixture was stirred at $80^{\circ} \mathrm{C}$ for 1 hour. The resulting mixture was filtered. The filtrate was concentrated under reduced pressure. This resulted in 2-[(E)-2-(4-aminophenyl)ethenyl]6-(4-methylpiperazin-1-yl)- $N$-[5-(oxetan-3-yl)-1H-pyrazol-3-yl]pyrimidin-4-amine (100 mg, 65\%) as a yellow crude solid. LCMS (ESI): [M+H]+: 433.2.<smiles>C=CC(=O)Nc1ccc(/C=C/c2nc(Nc3cc(C4COC4)[nH]n3)cc(N3CCN(C)CC3)n2)cc1</smiles>

\section{$\mathrm{N}$-[4-[(E)-2-[4-(4-Methylpiperazin-1-yl)-6-[[5-(oxetan-3-yl)-1H-pyrazol-3-yl]amino]pyrimidin-2-}

yl]ethenyl]phenyl]prop-2-enamide (Compound 11): To a mixture of 2-[(E)-2-(4-aminophenyl)ethenyl]-6(4-methylpiperazin-1-yl)- $N$-[5-(oxetan-3-yl)-1H-pyrazol-3-yl]pyrimidin-4-amine (50 mg, $0.116 \mathrm{mmol}, 1$ equiv) in DCM (5 mL) were added triethylamine ( $35 \mathrm{mg}, 0.347 \mathrm{mmol}, 3$ equiv) and acryloyl chloride (12 $\mathrm{mg}, 0.127 \mathrm{mmol}, 1.1$ equiv) dropwise at $0{ }^{\circ} \mathrm{C}$. The resulting mixture was stirred at $0{ }^{\circ} \mathrm{C}$ for 1 hour. The reaction was quenched with $\mathrm{MeOH}$. The resulting mixture was concentrated under vacuum. The residue 
was purified by reverse flash chromatography with the following conditions: Column: XBridge Shield RP18 OBD Column, 30*150mm, 5um; Mobile Phase A: Water (10MMOL/ $\mathrm{LH}_{4} \mathrm{HCO}_{3}$ ), Mobile Phase B: ACN; Flow rate: $60 \mathrm{~mL} / \mathrm{min}$; Gradient: $25 \mathrm{~B}$ to $49 \mathrm{~B}$ in $7 \mathrm{~min}$; detector, $220 \mathrm{~nm}$. This resulted in N-[4-[(E)-2-[4-(4methylpiperazin-1-yl)-6-[[5-(oxetan-3-yl)-1H-pyrazol-3-yl]amino]pyrimidin-2-yl]ethenyl]phenyl]prop-2-

enamide (2.4 mg, 4\%) as a light yellow solid. LCMS (ESI): $[\mathrm{M}+\mathrm{H}]^{+}:$: 487.2. ${ }^{1} \mathrm{H} \mathrm{NMR}\left(300 \mathrm{MHz}, \mathrm{CD}_{3} \mathrm{OD}\right) \delta 7.96$ - $7.46(\mathrm{~m}, 5 \mathrm{H}), 6.99-6.93(\mathrm{~m}, 1 \mathrm{H}), 6.53-6.31(\mathrm{~m}, 3 \mathrm{H}), 5.83-5.73(\mathrm{~m}, 1 \mathrm{H}), 5.10-4.99(\mathrm{~m}, 2 \mathrm{H}), 4.87-$ $4.77(\mathrm{~m}, 3 \mathrm{H}), 4.36-4.30(\mathrm{~m}, 1 \mathrm{H}), 3.71-3.65(\mathrm{~m}, 4 \mathrm{H}), 2.57-2.51(\mathrm{~m}, 4 \mathrm{H}), 2.35(\mathrm{~s}, 3 \mathrm{H})$.

\section{Compound 12:}
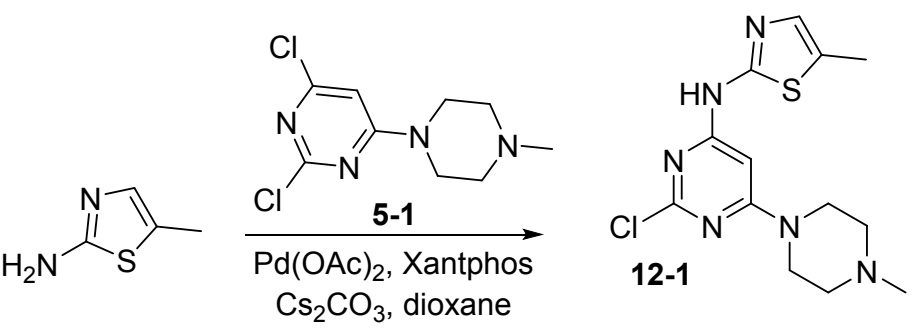

12-1

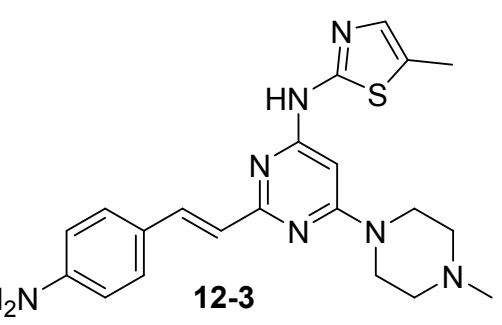

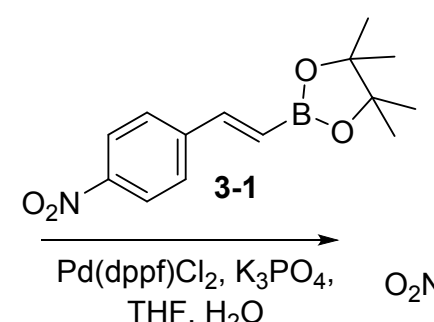

THF, $\mathrm{H}_{2} \mathrm{O}$
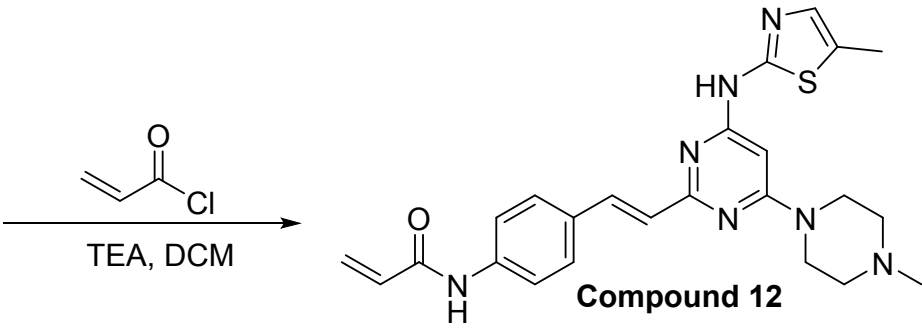

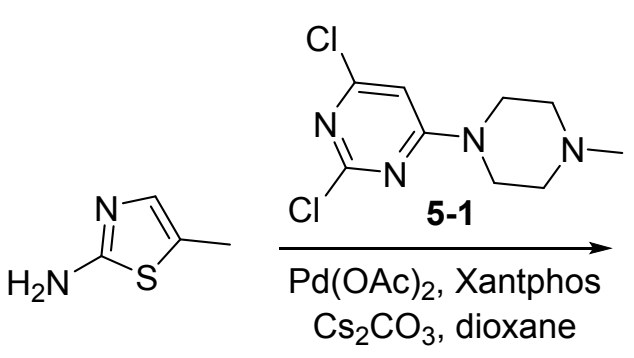

2-Chloro-N-(5-methyl-1,3-thiazol-2-yl)-6-(4-methylpiperazin-1-yl)pyrimidin-4-amine $\quad(12-1): \quad$ To a mixture of 5-methyl-1,3-thiazol-2-amine (660 mg, $5.781 \mathrm{mmol}, 1$ equiv) and 2,4-dichloro-6-(4methylpiperazin-1-yl)pyrimidine (1428 mg, $5.781 \mathrm{mmol}, 1$ equiv) in 1,4-dioxane (20 $\mathrm{mL})$ were added $\mathrm{Cs}_{2} \mathrm{CO}_{3}$ (2448 mg, $7.515 \mathrm{mmol}, 1.3$ equiv), XantPhos (668 mg, 1.156 mmol, 0.2 equiv) and Pd(OAc) 2 (389 $\mathrm{mg}, 1.734 \mathrm{mmol}, 0.3$ equiv). The resulting mixture was stirred at $50{ }^{\circ} \mathrm{C}$ overnight under nitrogen atmosphere. The resulting mixture was concentrated under vacuum. The residue was purified by silica gel column chromatography, eluting with $\mathrm{CH}_{2} \mathrm{Cl}_{2} / \mathrm{MeOH}(10 / 1)$ to afford 2-chloro- $N$-(5-methyl-1,3-thiazol-2- 
yl)-6-(4-methylpiperazin-1-yl)pyrimidin-4-amine (350 mg, 19\%) as a yellow solid. LCMS (ESI): [M+H]+: 325.0. Structures were assigned by $1 \mathrm{H}$ NMR analysis after de-chlorination.<smiles>[Z17][X]c1cc(Nc2ncc(C)s2)nc(Cl)n1</smiles><smiles>CC1(C)OB(/C=C/c2ccc([N+](=O)[O-])cc2)OC1(C)C</smiles><smiles>Cc1cnc(Nc2cc(N3CCN(C)CC3)nc(/C=C/c3ccc([N+](=O)[O-])cc3)n2)s1</smiles>

N-(5-Methyl-1,3-thiazol-2-yl)-6-(4-methylpiperazin-1-yl)-2-[(E)-2-(4-nitrophenyl)ethenyl]pyrimidin-4amine (12-2): To a mixture of 2-chloro- $N$-(5-methyl-1,3-thiazol-2-yl)-6-(4-methylpiperazin-1-yl)pyrimidin4-amine (350 mg, $1.077 \mathrm{mmol}, 1$ equiv) and 4,4,5,5-tetramethyl-2-[(E)-2-(4-nitrophenyl)ethenyl]-1,3,2dioxaborolane (592 mg, $2.155 \mathrm{mmol}, 2$ equiv) in THF $(5 \mathrm{~mL})$ were added $\mathrm{K}_{3} \mathrm{PO}_{4}(686 \mathrm{mg}, 3.232 \mathrm{mmol}, 3$ equiv) in $\mathrm{H}_{2} \mathrm{O}(0.5 \mathrm{~mL})$ and $\mathrm{Pd}(\mathrm{dppf}) \mathrm{Cl}_{2}(157 \mathrm{mg}, 0.215 \mathrm{mmol}, 0.2$ equiv). The resulting mixture was stirred at $80^{\circ} \mathrm{C}$ overnight under nitrogen atmosphere. The resulting mixture was concentrated under vacuum. The residue was purified by silica gel column chromatography, eluted with $\mathrm{CH}_{2} \mathrm{Cl}_{2} / \mathrm{MeOH}(10 / 1)$ to afford $N$-(5-methyl-1,3-thiazol-2-yl)-6-(4-methylpiperazin-1-yl)-2-[(E)-2-(4-nitrophenyl)ethenyl]pyrimidin-4amine (150 $\mathrm{mg}, 32 \%)$ as a yellow solid. LCMS (ESI): [M+H] $]^{+}: 438.3$.<smiles>[R22]c1cc([N+](=O)[O-])ccc1/C=C/c1nc(Nc2ncc(C)s2)cc(N2CCN(C)CC2)n1</smiles><smiles>Cc1cnc(Nc2cc(N3CCN(C)CC3)nc(/C=C/c3ccc(N)cc3)n2)s1</smiles>

2-[(E)-2-(4-Aminophenyl)ethenyl]-N-(5-methyl-1,3-thiazol-2-yl)-6-(4-methylpiperazin-1-yl)pyrimidin-4amine (12-3): To a mixture of $N$-(5-methyl-1,3-thiazol-2-yl)-6-(4-methylpiperazin-1-yl)-2-[(E)-2-(4nitrophenyl)ethenyl]pyrimidin-4-amine (150 mg, $0.343 \mathrm{mmol}, 1$ equiv) and iron (191 mg, $3.428 \mathrm{mmol}, 10$ equiv) in $\mathrm{EtOH}(5 \mathrm{~mL})$ was added saturated aqueous $\mathrm{NH}_{4} \mathrm{Cl}$ solution $(0.5 \mathrm{~mL})$. The resulting mixture was stirred at $80^{\circ} \mathrm{C}$ for 2 hours. The solids were filtered out and concentrated. The residue was purified by silica gel column chromatography, eluting with $\mathrm{CH}_{2} \mathrm{Cl}_{2} / \mathrm{MeOH}$ (5/1) to afford 2-[(E)-2-(4aminophenyl)ethenyl]- $N$-(5-methyl-1,3-thiazol-2-yl)-6-(4-methylpiperazin-1-yl)pyrimidin-4-amine $\quad$ (100 $\mathrm{mg}, 72 \%)$ as a yellow solid. LCMS (ESI): [M+H]+: 408.3. 
<smiles>[Z10]/C=C/c1ccc(N)cc1</smiles><smiles>C=CC(=O)Nc1ccc(/C=C/c2nc(Nc3ncc(C)s3)cc(N3CCN(C)CC3)n2)cc1</smiles>

$N$-[4-[(E)-2-[4-[(5-Methyl-1,3-thiazol-2-yl)amino]-6-(4-methylpiperazin-1-yl)pyrimidin-2-

yl]ethenyl]phenyl]prop-2-enamide (Compound 12): To a mixture of 2-[(E)-2-(4-aminophenyl)ethenyl]- $N$ (5-methyl-1,3-thiazol-2-yl)-6-(4-methylpiperazin-1-yl)pyrimidin-4-amine (100 mg, $0.245 \mathrm{mmol}, 1$ equiv) in DCM ( $3 \mathrm{~mL}$ ) were added triethylamine $(74 \mathrm{mg}, 0.736 \mathrm{mmol}, 3$ equiv) and acryloyl chloride $(22 \mathrm{mg}, 0.245$ mmol, 1 equiv) dropwise at $0{ }^{\circ} \mathrm{C}$. The resulting mixture was stirred at $0{ }^{\circ} \mathrm{C}$ for 1 hour. The reaction was quenched with $\mathrm{MeOH}$ at $0{ }^{\circ} \mathrm{C}$. The crude product was purified by Prep-HPLC with the following conditions: Column, YMC-Actus Triart C18, 20*250MM, 5um, 12nm; mobile phase, Water with $10 \mathrm{mmol} \mathrm{NH}_{4} \mathrm{HCO}_{3}$ and ACN (51.0\% ACN up to 81.0\% in $7 \mathrm{~min})$; Detector, UV 254nm. This resulted in N-[4-[(E)-2-[4-[(5-methyl1,3-thiazol-2-yl)amino]-6-(4-methylpiperazin-1-yl)pyrimidin-2-yl]ethenyl]phenyl]prop-2-enamide (24.5 $\mathrm{mg}, 22 \%)$ as a white solid. LCMS (ESI): $[\mathrm{M}+\mathrm{H}]^{+}:$462.3. ${ }^{1} \mathrm{H}$ NMR (300 MHz, CD $\left.{ }_{3} \mathrm{OD}\right) \delta 8.06-7.95(\mathrm{~m}, 1 \mathrm{H})$, $7.77-7.68(\mathrm{~m}, 2 \mathrm{H}), 7.67-7.58(\mathrm{~m}, 2 \mathrm{H}), 7.05-6.94(\mathrm{~m}, 2 \mathrm{H}), 6.49-6.33(\mathrm{~m}, 2 \mathrm{H}), 6.02(\mathrm{~s}, 1 \mathrm{H}), 5.85-5.75$ $(\mathrm{m}, 1 \mathrm{H}), 3.70(\mathrm{~s}, 4 \mathrm{H}), 2.61-2.53(\mathrm{~m}, 4 \mathrm{H}), 2.47-2.40(\mathrm{~m}, 3 \mathrm{H}), 2.37(\mathrm{~s}, 3 \mathrm{H})$.

\section{Compound 13:}

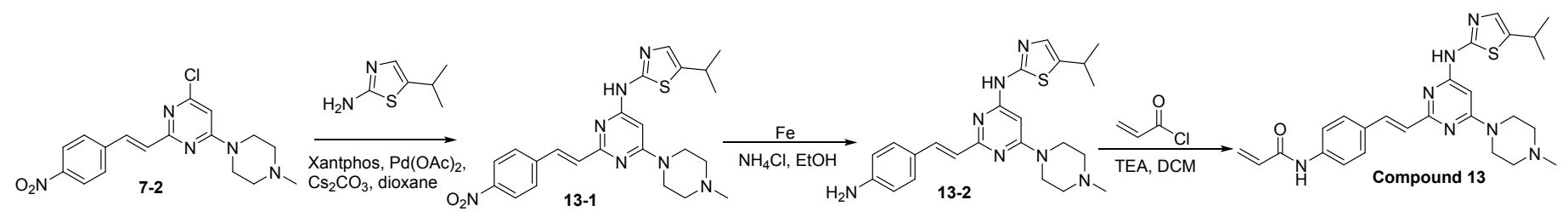

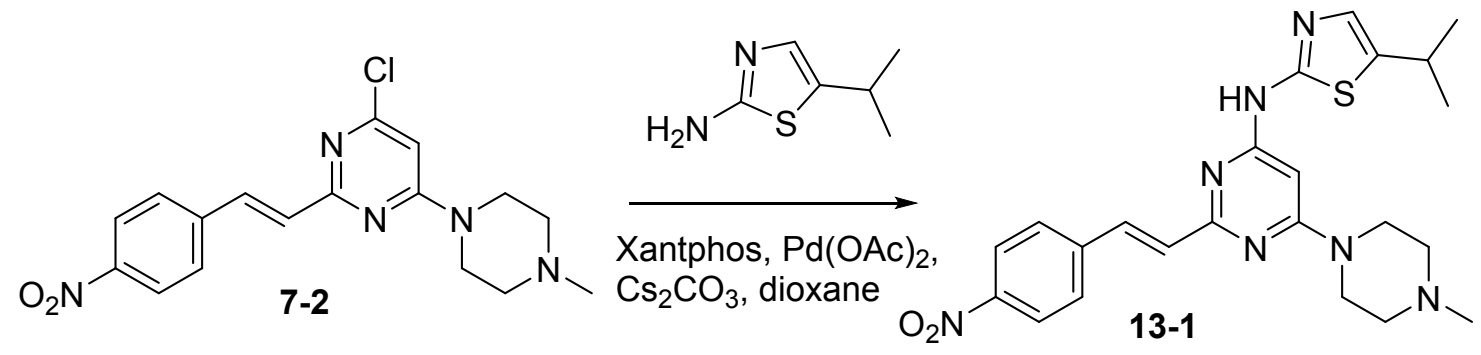

N-(5-Isopropyl-1,3-thiazol-2-yl)-6-(4-methylpiperazin-1-yl)-2-[(E)-2-(4-nitrophenyl)ethenyl]pyrimidin-4amine (13-1): To a mixture of 4-chloro-6-(4-methylpiperazin-1-yl)-2-[(E)-2-(4nitrophenyl)ethenyl]pyrimidine $\left(300 \mathrm{mg}, 0.834 \mathrm{mmol}, 1\right.$ equiv) in dioxane $\left(5 \mathrm{~mL}\right.$ ) was added $\mathrm{Cs}_{2} \mathrm{CO}_{3}$ (355 
$\mathrm{mg}, 1.090 \mathrm{mmol}, 1.3$ equiv), 5-isopropyl-1,3-thiazol-2-amine (177 mg, $1.245 \mathrm{mmol}, 1.5$ equiv), Xantphos (97 mg, $0.168 \mathrm{mmol}, 0.2$ equiv) and $\mathrm{Pd}(\mathrm{OAc})_{2}$ (38 mg, $0.169 \mathrm{mmol}, 0.2$ equiv). The resulting mixture was stirred at $50{ }^{\circ} \mathrm{C}$ for 12 hours. The resulting mixture was concentrated. The residue was applied onto a silica gel column eluting with dichloromethane/methanol (10/1). This resulted in $350 \mathrm{mg}(90 \%)$ of $\mathrm{N}$-(5isopropyl-1,3-thiazol-2-yl)-6-(4-methylpiperazin-1-yl)-2-[(E)-2-(4-nitrophenyl)ethenyl]pyrimidin-4-amine as a yellow solid. LCMS (ESI): [M+H $]^{+}: 466.2$.<smiles>CC(C)c1cnc(Nc2cc(N3CCN(C)CC3)nc(/C=C/c3ccc(N)cc3)n2)s1</smiles>

2-[(E)-2-(4-Aminophenyl)ethenyl]-N-(5-isopropyl-1,3-thiazol-2-yl)-6-(4-methylpiperazin-1-yl)pyrimidin4-amine (13-2): To a mixture of $N$-(5-isopropyl-1,3-thiazol-2-yl)-6-(4-methylpiperazin-1-yl)-2-[(E)-2-(4nitrophenyl)ethenyl]pyrimidin-4-amine (100 mg, 0.215 mmol, 1 equiv) in $\mathrm{EtOH}(5 \mathrm{~mL})$ was added $\mathrm{NH}_{4} \mathrm{Cl}$ aqueous ( $1 \mathrm{~mL}$ ) and iron (120 mg, $2.149 \mathrm{mmol}, 10$ equiv). The resulting mixture was stirred at $90{ }^{\circ} \mathrm{C}$ for 1 hour. The resulting mixture was concentrated. The residue was applied onto a silica gel column eluting with dichloromethane/methanol (10/1). This resulted in $60 \mathrm{mg}$ (64\%) of 2-[(E)-2-(4-aminophenyl)ethenyl]$\mathrm{N}$-(5-isopropyl-1,3-thiazol-2-yl)-6-(4-methylpiperazin-1-yl)pyrimidin-4-amine as a yellow solid. LCMS (ESI): $[\mathrm{M}+\mathrm{H}]^{+}:$:36.2.<smiles>C=CC(=O)C(C)(C)C(=O)OCC(=O)Nc1ccc(/C=C/C=C/c2nc(Nc3ncc(C(C)C)s3)cc(N3CCN(C)CC3)n2)cc1</smiles>

$N$-[4-[(E)-2-[4-[(5-Isopropyl-1,3-thiazol-2-yl)amino]-6-(4-methylpiperazin-1-yl)pyrimidin-2-

yl]ethenyl]phenyl]prop-2-enamide (Compound 13): To a mixture of 2-[(E)-2-(4-aminophenyl)ethenyl]- $N$ (5-isopropyl-1,3-thiazol-2-yl)-6-(4-methylpiperazin-1-yl)pyrimidin-4-amine (60 mg, $0.138 \mathrm{mmol}, 1$ equiv) in DCM ( $5 \mathrm{~mL}$ ) was added acryloyl chloride $(13 \mathrm{mg}, 0.144 \mathrm{mmol}, 1$ equiv). The resulting mixture was stirred at $0{ }^{\circ} \mathrm{C}$ for 15 minutes. The resulting mixture was concentrated. The crude product was purified by following condition: Column: XBridge Shield RP18 OBD Column, 30*150mm, 5um; Mobile Phase A: Water $\left(10 \mathrm{mmol} / \mathrm{L} \mathrm{NH}_{4} \mathrm{HCO}_{3}\right)$, Mobile Phase B: ACN; Flow rate: $60 \mathrm{~mL} / \mathrm{min}$; Detector, UV 254/220 nm. This 
resulted in $6 \mathrm{mg}$ (9\%) of $\mathrm{N}$-[4-[(E)-2-[4-[(5-isopropyl-1,3-thiazol-2-yl)amino]-6-(4-methylpiperazin-1yl)pyrimidin-2-yl] ethenyl]phenyl]prop-2-enamide as a white solid. LCMS (ESI): [M+H] ${ }^{+}: 490.2 .{ }^{1} \mathrm{H}$ NMR (300 $\left.\mathrm{MHz}, \mathrm{CD}_{3} \mathrm{COCD}_{3}\right): \delta$ 9.88-9.75 $(\mathrm{m}, 1 \mathrm{H}), 9.49(\mathrm{~s}, 1 \mathrm{H})$, 8.11-8.06 $(\mathrm{m}, 1 \mathrm{H}), 7.83-7.80(\mathrm{~m}, 2 \mathrm{H})$, 7.66-7.63 $(\mathrm{m}, 2 \mathrm{H})$, 7.03-6.98 (m, 2H), 6.51-6.32 (m, 2H), $6.18(\mathrm{~s}, 1 \mathrm{H}), 5.75-5.71(\mathrm{~m}, 1 \mathrm{H}), 3.66-3.63(\mathrm{~m}, 4 \mathrm{H}), 3.20-3.16(\mathrm{~m}, 1 \mathrm{H})$, 2.44-2.27 (m, $4 \mathrm{H}), 2.24(\mathrm{~s}, 3 \mathrm{H}), 1.37(\mathrm{~d}, J=6.0 \mathrm{~Hz}, 6 \mathrm{H})$.

\section{Compound 14:}
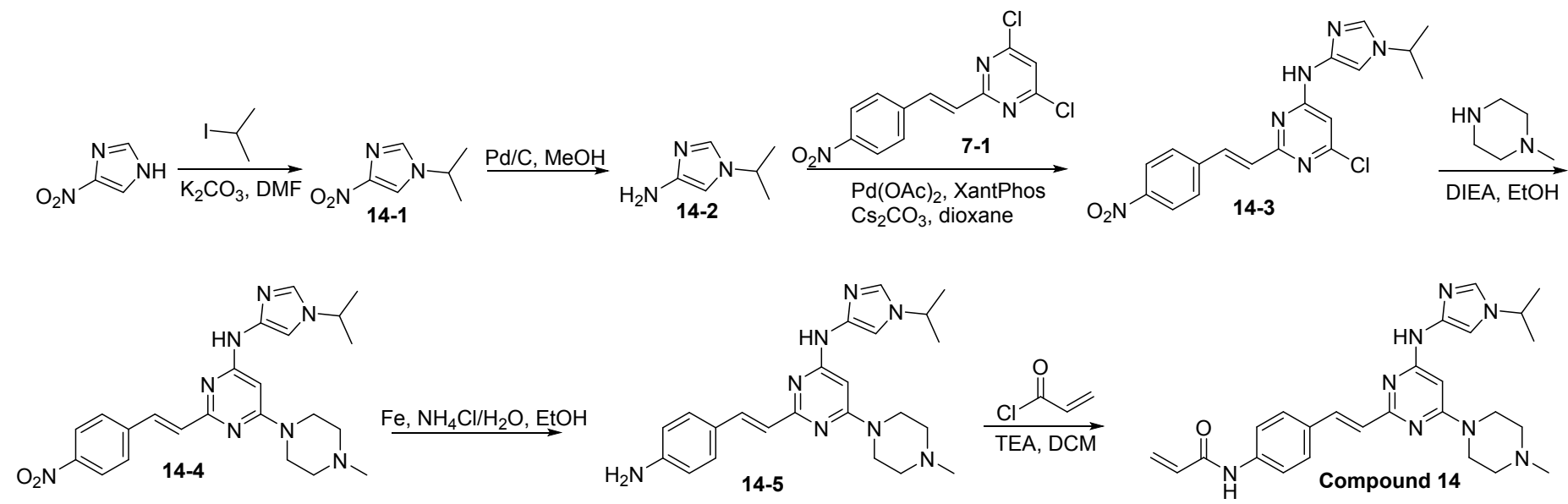

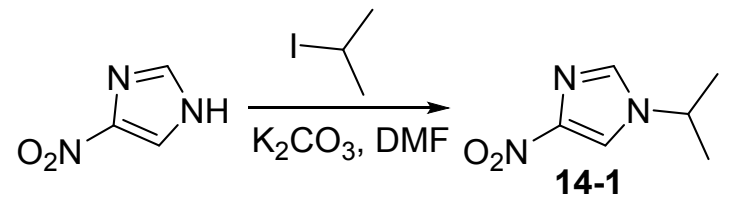

1-Isopropyl-4-nitroimidazole (14-1): To a mixture of 4-nitroimidazole (5 g, $44.218 \mathrm{mmol}, 1$ equiv) and 2iodopropane ( $9.8 \mathrm{~g}, 57.483 \mathrm{mmol}, 1.3$ equiv) in DMF ( $30 \mathrm{~mL}$ ) was added $\mathrm{K}_{2} \mathrm{CO}_{3}(9.2 \mathrm{~g}, 66.327 \mathrm{mmol}, 1.5$ equiv). The resulting mixture was stirred at $50{ }^{\circ} \mathrm{C}$ for 5 hours. The resulting mixture was diluted with water $(20 \mathrm{~mL})$. The aqueous layer was extracted with EtOAc. The organic layer was dried with sodium sulfate and concentrated under vacuum. The residue was purified by silica gel column chromatography, eluting with PE/EtOAc (3/1). This resulted in 1-isopropyl-4-nitroimidazole (4 g, 58\%) as a white solid. LCMS (ESI): $[\mathrm{M}+\mathrm{H}]^{+}: 156.0$.<smiles>CC(C)n1cnc(N)c1</smiles>

1-Isopropylimidazol-4-amine (14-2): To a mixture of 1-isopropyl-4-nitroimidazole $(2 \mathrm{~g}, 12.890 \mathrm{mmol}, 1$ equiv) in $\mathrm{MeOH}(60 \mathrm{~mL}$ ) was added $\mathrm{Pd} / \mathrm{C}(200 \mathrm{mg})$. The resulting mixture was stirred at room temperature 
for overnight under hydrogen atmosphere. The resulting mixture was filtered. The filtrate was concentrated under reduced pressure. This resulted in 1-isopropylimidazol-4-amine $(1.7 \mathrm{~g}, 95 \%)$ as a yellow oil. LCMS (ESI): [M+H]+: 126.1.<smiles>CC(C)n1cnc(Nc2cc(Cl)nc(/C=C/c3ccc([N+](=O)[O-])cc3)n2)c1</smiles>

6-Chloro-N-(1-isopropylimidazol-4-yl)-2-[(E)-2-(4-nitrophenyl)ethenyl]pyrimidin-4-amine (14-3): To a mixture of 1-isopropylimidazol-4-amine (250 mg, $1.997 \mathrm{mmol}, 1$ equiv) and 4,6-dichloro-2-[(E)-2-(4nitrophenyl)ethenyl]pyrimidine (592 mg, $1.997 \mathrm{mmol}, 1$ equiv) and $\mathrm{Cs}_{2} \mathrm{CO}_{3}$ ( $846 \mathrm{mg}, 2.596 \mathrm{mmol}, 1.3$ equiv) in dioxane $\left(5 \mathrm{~mL}\right.$ ) were added XantPhos $\left(231 \mathrm{mg}, 0.399 \mathrm{mmol}, 0.2\right.$ equiv) and $\mathrm{Pd}(\mathrm{OAc})_{2}(45 \mathrm{mg}, 0.200$ mmol, 0.1 equiv). The resulting mixture was stirred at $35^{\circ} \mathrm{C}$ for 2 hours under nitrogen atmosphere. The resulting mixture was concentrated under vacuum. The residue was purified by silica gel column chromatography, eluting with $\mathrm{CH}_{2} \mathrm{Cl}_{2} / \mathrm{MeOH}$ (20/1). This resulted in 6-chloro- $\mathrm{N}$-(1-isopropylimidazol-4-yl)2-[(E)-2-(4-nitrophenyl)ethenyl]pyrimidin-4-amine (400 $\mathrm{mg}, 52 \%)$ as a yellow solid. LCMS (ESI): [M+H] $]^{+}$ 385.1 .<smiles>CC(C)n1cnc(Nc2cc(Cl)nc(/C=C/c3ccc([N+](=O)[O-])cc3)n2)c1</smiles>

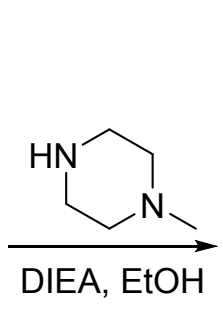<smiles>CC(C)n1cnc(Nc2cc(N3CCN(C)CC3)nc(/C=C/c3ccc([N+](=O)[O-])cc3)n2)c1</smiles>

N-(1-Isopropylimidazol-4-yl)-6-(4-methylpiperazin-1-yl)-2-[(E)-2-(4-nitrophenyl)ethenyl]pyrimidin-4amine (14-4): To a mixture of 6-chloro- $N$-(1-isopropylimidazol-4-yl)-2-[(E)-2-(4nitrophenyl)ethenyl]pyrimidin-4-amine (380 mg, $0.987 \mathrm{mmol}, 1$ equiv) in EtOH (15 mL) were added 1methyl-piperazine (198 mg, $1.975 \mathrm{mmol}, 2$ equiv) and diisopropylethylamine (383 mg, $2.962 \mathrm{mmol}, 3$ equiv) dropwise. The resulting mixture was stirred at $100{ }^{\circ} \mathrm{C}$ overnight under nitrogen atmosphere. The resulting mixture was concentrated under vacuum. The residue was purified by silica gel column chromatography, eluting with $\mathrm{CH}_{2} \mathrm{Cl}_{2} / \mathrm{MeOH}$ (10/1). This resulted in $\mathrm{N}$-(1-isopropylimidazol-4-yl)-6-(4methylpiperazin-1-yl)-2-[(E)-2-(4-nitrophenyl)ethenyl]pyrimidin-4-amine (230 mg, 52\%) as a yellow solid. LCMS (ESI): [M+H]+: 449.2. 
<smiles>CC(C)n1cnc(Nc2cc(N3CCN(C)CC3)nc(/C=C/c3ccc([N+](=O)[O-])cc3)n2)c1</smiles><smiles>CC(C)n1cnc(Nc2cc(N3CCN(C)CC3)nc(/C=C/c3ccc(N)cc3)n2)c1</smiles>

2-[(E)-2-(4-Aminophenyl)ethenyl]- $N$-(1-isopropylimidazol-4-yl)-6-(4-methylpiperazin-1-yl)pyrimidin-4amine (14-5): To a mixture of $N$-(1-isopropylimidazol-4-yl)-6-(4-methylpiperazin-1-yl)-2-[(E)-2-(4nitrophenyl)ethenyl]pyrimidin-4-amine ( $230 \mathrm{mg}, 0.513 \mathrm{mmol}, 1$ equiv) in EtOH ( $1 \mathrm{~mL}$ ) were added iron (287 mg, $5.128 \mathrm{mmol}, 10$ equiv) and saturated aqueous $\mathrm{NH}_{4} \mathrm{Cl}(10 \mathrm{~mL}$ ) solution. The resulting mixture was stirred at $80^{\circ} \mathrm{C}$ for 1 hour. The resulting mixture was filtered. The filtrate was concentrated under reduced pressure. This resulted in 2-[(E)-2-(4-aminophenyl)ethenyl]- $N$-(1-isopropylimidazol-4-yl)-6-(4methylpiperazin-1-yl)pyrimidin-4-amine (200 mg, 93\%) as a yellow solid. LCMS (ESI): [M+H]+: 419.2.<smiles>CC(C)n1cnc(Nc2cc(N3CCN(C)CC3)nc(/C=C/c3ccc(N)cc3)n2)c1</smiles><smiles>C=CC(=O)Cl</smiles><smiles>C=CC(=O)Nc1ccc(/C=C/c2nc(Nc3cn(C(C)C)cn3)cc(N3CCN(C)CC3)n2)cc1</smiles>

$N$-[4-[(E)-2-[4-[(1-Isopropylimidazol-4-yl)amino]-6-(4-methylpiperazin-1-yl)pyrimidin-2-

yl]ethenyl]phenyl]prop-2-enamide (Compound 14): To a mixture of 2-[(E)-2-(4-aminophenyl)ethenyl]- $N$ (1-isopropylimidazol-4-yl)-6-(4-methylpiperazin-1-yl)pyrimidin-4-amine (180 mg, $0.430 \mathrm{mmol}, 1$ equiv) in DCM (10 mL) were added triethylamine $(131 \mathrm{mg}, 1.290 \mathrm{mmol}, 3$ equiv) and acryloyl chloride (43 mg, 0.473 mmol, 1.1 equiv) dropwise at $0{ }^{\circ} \mathrm{C}$. The resulting mixture was stirred at $0{ }^{\circ} \mathrm{C}$ for 1 hour. The reaction was quenched with $\mathrm{MeOH}$ at $0{ }^{\circ} \mathrm{C}$. The resulting mixture was concentrated under vacuum. The residue was purified by Prep-TLC $\left(\mathrm{CH}_{2} \mathrm{Cl}_{2} / \mathrm{MeOH}=5 / 1\right)$. The residue was purified by reverse flash chromatography with the following conditions: column: Xselect CSH OBD Column 30*150mm 5um, n; Mobile Phase A: Water (10MMOL/L $\mathrm{NH}_{4} \mathrm{HCO}_{3}$ ), Mobile Phase B: ACN; Flow rate: $60 \mathrm{~mL} / \mathrm{min}$; Gradient: 2 B to 2 B in $1 \mathrm{~min} ; 254 / 220$ $\mathrm{nm}$; RT1:7 min. This resulted in N-[4-[(E)-2-[4-[(1-isopropylimidazol-4-yl)amino]-6-(4-methylpiperazin-1yl)pyrimidin-2-yl]ethenyl]phenyl]prop-2-enamide (9 $\mathrm{mg}, 4 \%)$ as a light yellow solid. LCMS (ESI): [M+H]+: 473.2. ${ }^{1} \mathrm{H}$ NMR (300 MHz, CD $\left.{ }_{3} \mathrm{OD}\right) \delta 7.84-7.73(\mathrm{~m}, 1 \mathrm{H}), 7.69(\mathrm{~d}, J=8.7 \mathrm{~Hz}, 2 \mathrm{H}), 7.62-7.51(\mathrm{~m}, 3 \mathrm{H}), 7.31$ $(\mathrm{d}, J=1.5 \mathrm{~Hz}, 1 \mathrm{H}), 6.98-6.87(\mathrm{~m}, 1 \mathrm{H}), 6.53-6.38(\mathrm{~m}, 1 \mathrm{H}), 6.43-6.30(\mathrm{~m}, 1 \mathrm{H}), 5.88(\mathrm{~s}, 1 \mathrm{H}), 5.83-5.73$ $(\mathrm{m}, 1 \mathrm{H}), 4.52-4.37(\mathrm{~m}, 1 \mathrm{H}), 3.68-3.59(\mathrm{~m}, 4 \mathrm{H}), 2.58-2.48(\mathrm{~m}, 4 \mathrm{H}), 2.34(\mathrm{~s}, 3 \mathrm{H}), 1.54(\mathrm{~d}, J=6.6 \mathrm{~Hz}, 6 \mathrm{H})$.

\section{Compound 15:}



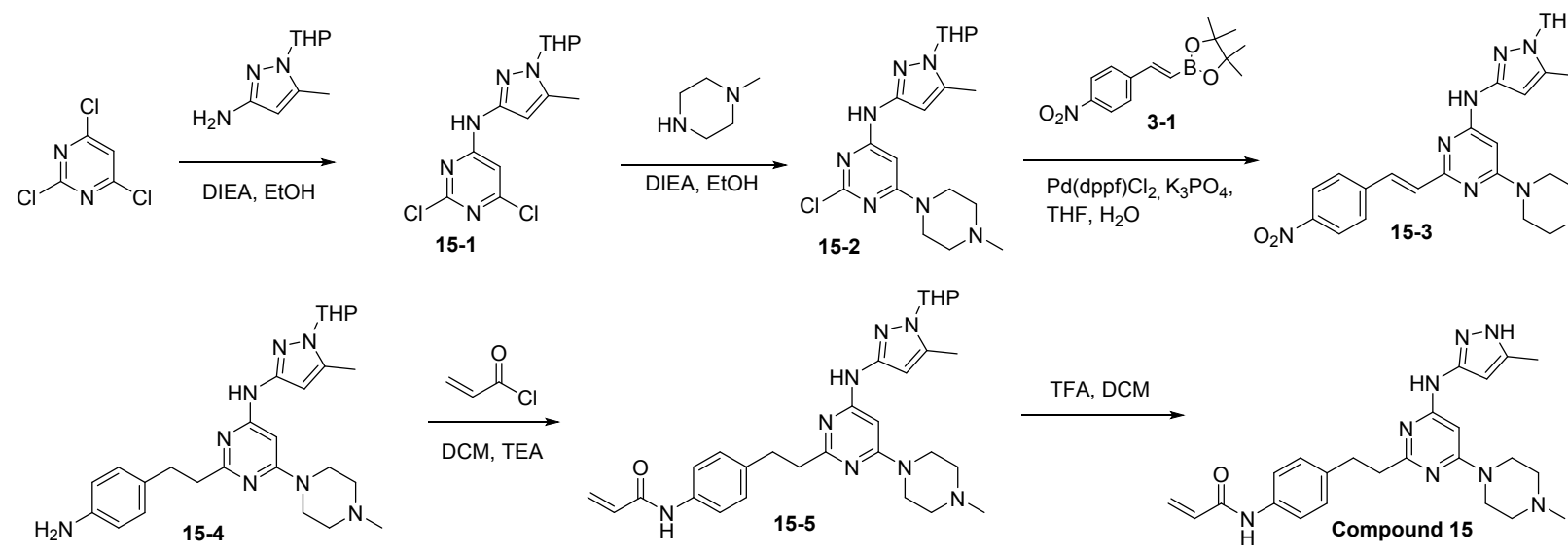<smiles>Clc1cc(Cl)nc(Cl)n1</smiles>

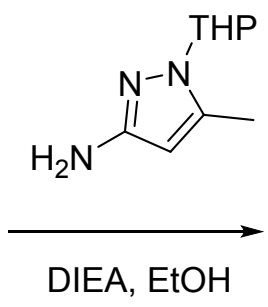<smiles>Cc1cc(Nc2cc(Cl)nc(Cl)n2)nn1P</smiles>

15-1

2,6-Dichloro- $N$-[5-methyl-1-(oxan-2-yl)pyrazol-3-yl]pyrimidin-4-amine (15-1): To a mixture of 2,4,6trichloropyrimidine (2 g, $10.904 \mathrm{mmol}, 1$ equiv) in EtOH (15 mL) were added 5-methyl-1-(oxan-2yl)pyrazol-3-amine ( $2 \mathrm{~g}, 10.904 \mathrm{mmol}, 1$ equiv) and diisopropylethylamine (1.4 g, $10.904 \mathrm{mmol}, 1$ equiv) at $0{ }^{\circ} \mathrm{C}$. The resulting mixture was stirred at room temperature overnight. The precipitated solids were collected by filtration and washed with methanol $(100 \mathrm{~mL})$. This resulted in 2,6-dichloro- $N$-[5-methyl-1(oxan-2-yl)pyrazol-3-yl]pyrimidin-4-amine $(2.2 \mathrm{~g}, 58 \%)$ as a white solid. LCMS (ESI): [M+H]+: 328.0. Structures were assigned by $1 \mathrm{H}$ NMR analysis after de-chlorination.<smiles>Cc1cc(Nc2cc(Cl)nc(Cl)n2)nn1P</smiles>

15-1
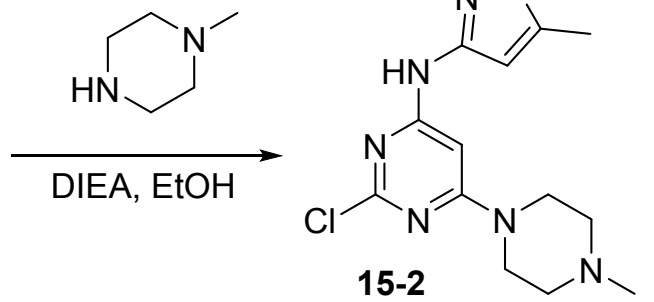

2-Chloro-N-[5-methyl-1-(oxan-2-yl)pyrazol-3-yl]-6-(4-methylpiperazin-1-yl)pyrimidin-4-amine (15-2): To a mixture of 2,6-dichloro- $N$-[5-methyl-1-(oxan-2-yl)pyrazol-3-yl]pyrimidin-4-amine (960 mg, 2.925 mmol, 1 equiv) and 1-methylpiperazine (293 mg, $2.925 \mathrm{mmol}, 1$ equiv) in EtOH (10 mL) was added 
diisopropylethylamine (567 mg, $4.388 \mathrm{mmol}, 1.5$ equiv). The resulting mixture was stirred at $80{ }^{\circ} \mathrm{C}$ overnight. The resulting mixture was concentrated under vacuum. The residue was purified by silica gel column chromatography, eluting with $\mathrm{CH}_{2} \mathrm{Cl}_{2} / \mathrm{MeOH}$ (10/1). This resulted in 2-chloro- $N$-[5-methyl-1(oxan-2-yl)pyrazol-3-yl]-6-(4-methylpiperazin-1-yl)pyrimidin-4-amine (150 mg, 12\%) as a white solid. LCMS (ESI): $[\mathrm{M}+\mathrm{H}]^{+}:$392.2. Structures were assigned by $1 \mathrm{H}$ NMR analysis after de-chlorination.

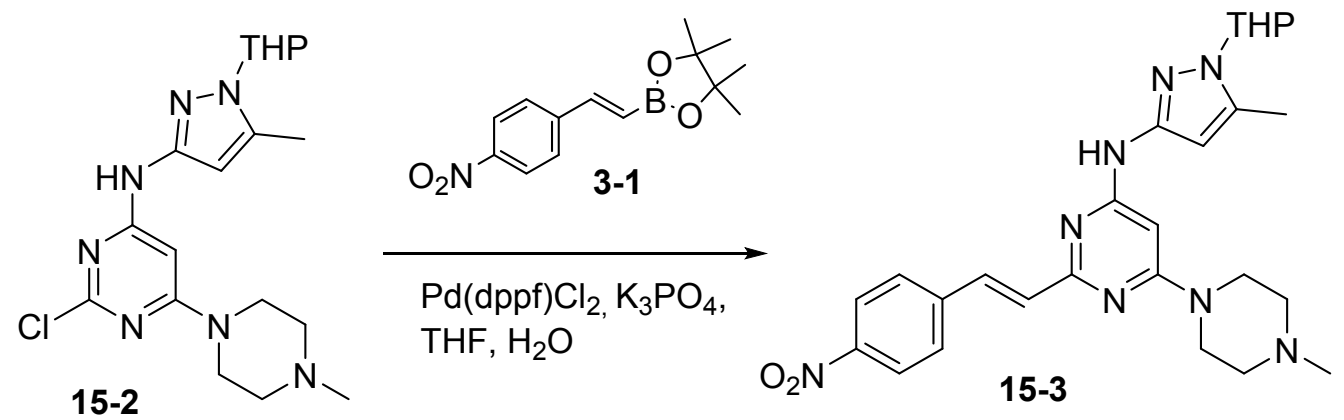

$N$-[5-Methyl-1-(oxan-2-yl)pyrazol-3-yl]-6-(4-methylpiperazin-1-yl)-2-[(E)-2-(4nitrophenyl)ethenyl]pyrimidin-4-amine (15-3): To a mixture of 2-chloro- $N$-[5-methyl-1-(oxan-2yl)pyrazol-3-yl]-6-(4-methylpiperazin-1-yl)pyrimidin-4-amine (100 mg, $0.255 \mathrm{mmol}, 1$ equiv) and 4,4,5,5tetramethyl-2-[(E)-2-(4-nitrophenyl)ethenyl]-1,3,2-dioxaborolane (105 mg, $0.383 \mathrm{mmol}, 1.5$ equiv) in THF $(1 \mathrm{~mL})$ were added $\mathrm{K}_{3} \mathrm{PO}_{4}\left(163 \mathrm{mg}, 0.766 \mathrm{mmol}, 3\right.$ equiv) in $\mathrm{H}_{2} \mathrm{O}(0.2 \mathrm{~mL})$ and $\mathrm{Pd}(\mathrm{dppf}) \mathrm{Cl}_{2}(19 \mathrm{mg}, 0.026$ mmol, 0.10 equiv). The resulting mixture was stirred at $80^{\circ} \mathrm{C}$ overnight. The mixture was concentrated under vacuum. The residue was purified by silica gel column chromatography, eluting with $\mathrm{CH}_{2} \mathrm{Cl}_{2} / \mathrm{MeOH}$ (10/1). This resulted in $N$-[5-methyl-1-(oxan-2-yl)pyrazol-3-yl]-6-(4-methylpiperazin-1-yl)-2-[(E)-2-(4nitrophenyl)ethenyl]pyrimidin-4-amine (120 mg, 93\%) as a yellow solid. LCMS (ESI): [M+H]+: 505.2 .<smiles>Cc1cc(Nc2cc(N3CCN(C)CC3)nc(CCc3ccc(N)cc3)n2)nc(Nc2cc(N3CCN(C)CC3)nc(/C=C/c3ccc([N+](=O)[O-])cc3)n2)n1</smiles>

\section{2-[2-(4-Aminophenyl)ethyl]-N-[5-methyl-1-(oxan-2-yl)pyrazol-3-yl]-6-(4-methylpiperazin-1-}

yl)pyrimidin-4-amine (15-4): To a mixture of $N$-[5-methyl-1-(oxan-2-yl)pyrazol-3-yl]-6-(4methylpiperazin-1-yl)-2-[(E)-2-(4-nitrophenyl)ethenyl]pyrimidin-4-amine (120 mg, $0.238 \mathrm{mmol}, 1$ equiv) in THF (10 mL) was added Pd/C (120 mg, $1.128 \mathrm{mmol}, 4.74$ equiv). The resulting mixture was stirred at room temperature overnight under a hydrogen atmosphere. The resulting mixture was filtered. The filtrate was concentrated under reduced pressure. The residue was purified by silica gel column 
chromatography, eluting with $\mathrm{CH}_{2} \mathrm{Cl}_{2} / \mathrm{MeOH}$ (10/1). This resulted in 2-[2-(4-aminophenyl)ethyl]- $\mathrm{N}$-[5methyl-1-(oxan-2-yl)pyrazol-3-yl]-6-(4-methylpiperazin-1-yl)pyrimidin-4-amine (80 $\mathrm{mg}, 71 \%$ ) as a yellow solid. LCMS (ESI): [M+H]+: 477.2.<smiles>Cc1cc(Nc2cc(N3CCN(C)CC3)nc(CCc3ccc(N)cc3)n2)nn1[TlH]</smiles><smiles>C=CC(=O)Cl</smiles>

\section{N-[4-[2-(4-[[5-Methyl-1-(oxan-2-yl)pyrazol-3-yl]amino]-6-(4-methylpiperazin-1-yl)pyrimidin-2-}

yl)ethyl]phenyl]prop-2-enamide (15-5): To a mixture of 2-[2-(4-aminophenyl)ethyl]- $N$-[5-methyl-1-(oxan2-yl)pyrazol-3-yl]-6-(4-methylpiperazin-1-yl)pyrimidin-4-amine (70 mg, $0.147 \mathrm{mmol}, 1$ equiv) in DCM (10 $\mathrm{mL}$ ) were added triethylamine ( $45 \mathrm{mg}, 0.441 \mathrm{mmol}, 3$ equiv) and acryloyl chloride ( $15 \mathrm{mg}, 0.162 \mathrm{mmol}$, 1.1 equiv). The resulting mixture was stirred at $0{ }^{\circ} \mathrm{C}$ for 1 hour. The reaction was quenched with $\mathrm{MeOH}$ at $0{ }^{\circ} \mathrm{C}$. The resulting mixture was concentrated under vacuum. This resulted in $\mathrm{N}$-[4-[2-(4-[[5-methyl-1(oxan-2-yl)pyrazol-3-yl]amino]-6-(4-methylpiperazin-1-yl)pyrimidin-2-yl)ethyl]phenyl]prop-2-enamide (70 mg, 90\%) as a yellow crude solid. LCMS (ESI): [M+H]+: 531.4.<smiles>C=CC(=O)Nc1ccc(CCc2nc(CCc3ccc(Nc4cc(C(C)(F)F)n([Tl])n4)cc3)cc(Nc3cc(CCc4nc(Nc5cc(C)[nH]n5)cc(N5CCN(C)CC5)n4)cc(N4CCN(C)CC4)n3)n2)cc1</smiles>

$N$-[4-(2-[4-[(5-Methyl-1H-pyrazol-3-yl)amino]-6-(4-methylpiperazin-1-yl)pyrimidin-2yl]ethyl)phenyl]prop-2-enamide (Compound 15): To a mixture of $\mathrm{N}$-[4-[2-(4-[[5-methyl-1-(oxan-2yl)pyrazol-3-yl]amino]-6-(4-methylpiperazin-1-yl)pyrimidin-2-yl)ethyl]phenyl]prop-2-enamide (70 $\mathrm{mg}$, $0.132 \mathrm{mmol}, 1$ equiv) in DCM $(5 \mathrm{~mL})$ were added TFA $(2 \mathrm{~mL})$. The resulting mixture was stirred at room temperature for 4 hours. The resulting mixture was concentrated under vacuum. The residue was purified by $\mathrm{C} 18$ reverse phase column with $\mathrm{ACN} / \mathrm{H}_{2} \mathrm{O}(1 / 3)$. This resulted in $\mathrm{N}$-[4-(2-[4-[(5-methyl- $1 \mathrm{H}$-pyrazol-3yl)amino]-6-(4-methylpiperazin-1-yl)pyrimidin-2-yl]ethyl)phenyl]prop-2-enamide (23 $\mathrm{mg}, 39 \%$ ) as a white solid. LCMS (ESI): $[\mathrm{M}+\mathrm{H}]^{+}:$: 447.25. ${ }^{1} \mathrm{H}$ NMR $\left(300 \mathrm{MHz}, \mathrm{CD}_{3} \mathrm{OD}\right) \delta 7.50$ (d, $\left.J=8.4 \mathrm{~Hz}, 2 \mathrm{H}\right), 7.17$ (d, $J=8.4 \mathrm{~Hz}$, 
$2 \mathrm{H}), 6.49-6.26(\mathrm{~m}, 2 \mathrm{H}), 6.22-6.16(\mathrm{~m}, 1 \mathrm{H}), 5.94-5.88(\mathrm{~m}, 1 \mathrm{H}), 5.79-5.69(\mathrm{~m}, 1 \mathrm{H}), 3.64-3.55(\mathrm{~m}, 4 \mathrm{H})$, $3.10-2.99(\mathrm{~m}, 2 \mathrm{H}), 2.94-2.83(\mathrm{~m}, 2 \mathrm{H}), 2.55-2.45(\mathrm{~m}, 4 \mathrm{H}), 2.33(\mathrm{~s}, 3 \mathrm{H}), 2.26(\mathrm{~s}, 3 \mathrm{H})$.

\section{Compound 16:}
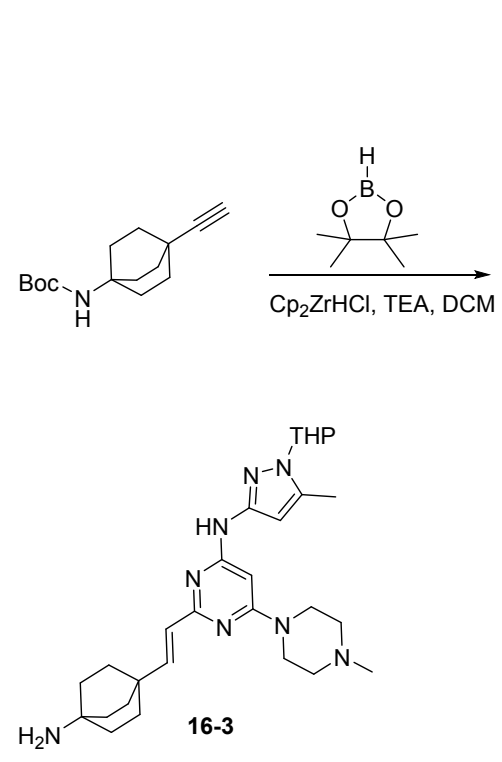
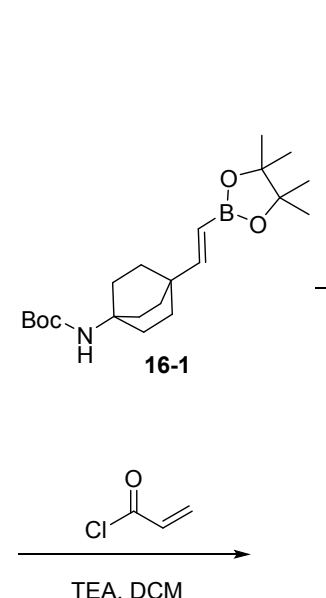

TEA, DCM
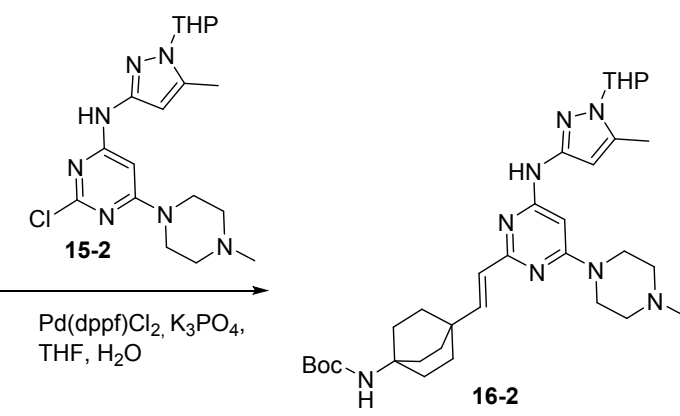

TFA, DCM

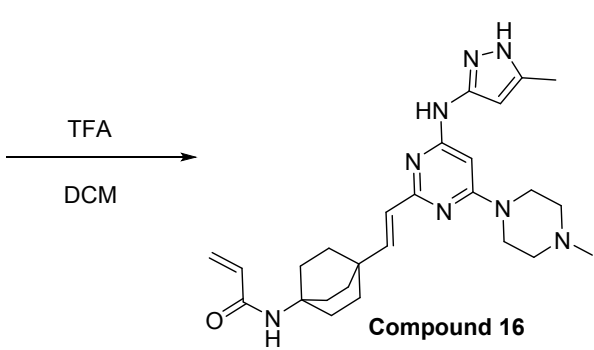<smiles>C#CC12CCC(NC(=O)OC(C)(C)C)(CC1)CC2</smiles>

$\mathrm{Cp}_{2} \mathrm{ZrHCl}, \mathrm{TEA}, \mathrm{DCM}$<smiles>CC(C)(C)OC(=O)NC12CCC(/C=C/B3OC(C)(C)C(C)(C)O3)(CC1)CC2</smiles>

16-1

tert-Butyl $\quad N$-[4-[(E)-2-(4,4,5,5-tetramethyl-1,3,2-dioxaborolan-2-yl)ethenyl]bicyclo[2.2.2]octan-1yl]carbamate (16-1): To a mixture of tert-butyl $N$-[4-ethynylbicyclo[2.2.2]octan-1-yl]carbamate (1 g, $4.010 \mathrm{mmol}, 1$ equiv) and triethylamine $(203 \mathrm{mg}, 2.005 \mathrm{mmol}, 0.5$ equiv) in DCM (10 mL) were added $\mathrm{Cp}_{2} \mathrm{ZrHCl}$ (399 mg, $2.005 \mathrm{mmol}, 0.5$ equiv) and 4,4,5,5-tetramethyl-1,3,2-dioxaborolane (2.57 g, 20.052 mmol, 5 equiv). The resulting mixture was stirred at room temperature overnight under nitrogen atmosphere. The reaction was quenched with $\mathrm{MeOH}$. The resulting mixture was concentrated under vacuum. The residue was purified by silica gel column chromatography, eluting with $\mathrm{CH}_{2} \mathrm{Cl}_{2} / \mathrm{MeOH}(99 / 1)$. This resulted in tert-butyl $N$-[4-[(E)-2-(4,4,5,5-tetramethyl-1,3,2-dioxaborolan-2yl)ethenyl]bicyclo[2.2.2] octan-1-yl]carbamate (1 g, 66\%) as a white solid. LCMS (ESI): [M-tBu+1] $]^{+}: 322.3$. 


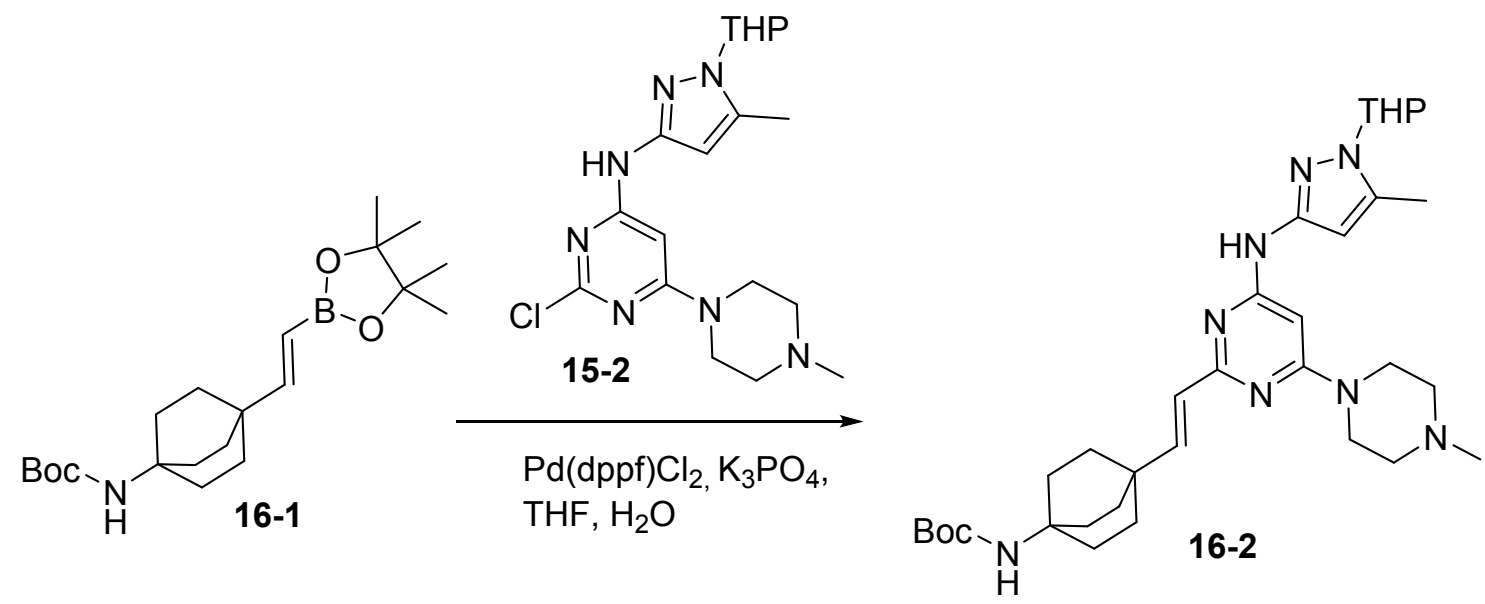

tert-Butyl N-[4-[(E)-2-(4-[[5-methyl-1-(oxan-2-yl)pyrazol-3-yl]amino]-6-(4-methylpiperazin-1yl)pyrimidin-2-yl)ethenyl]bicyclo[2.2.2]octan-1-yl]carbamate (16-2): To a mixture of 2-chloro- $N$-[5methyl-1-(oxan-2-yl)pyrazol-3-yl]-6-(4-methylpiperazin-1-yl)pyrimidin-4-amine (250 mg, $0.638 \mathrm{mmol}, 1$ equiv) and tert-butyl $\quad N$-[4-[(E)-2-(4,4,5,5-tetramethyl-1,3,2-dioxaborolan-2yl)ethenyl]bicyclo[2.2.2] octan-1-yl]carbamate $(241 \mathrm{mg}, 0.638 \mathrm{mmol}, 1$ equiv) in THF (5 mL) were added $\mathrm{K}_{3} \mathrm{PO}_{4}$ (406 mg, $1.914 \mathrm{mmol}, 3$ equiv) in $\mathrm{H}_{2} \mathrm{O}(0.5 \mathrm{~mL})$ and $\mathrm{Pd}(\mathrm{dppf}) \mathrm{Cl}_{2}$ (47 mg, $0.064 \mathrm{mmol}, 0.1$ equiv). The resulting mixture was stirred at $80{ }^{\circ} \mathrm{C}$ overnight under nitrogen atmosphere. The resulting mixture was concentrated under vacuum. The residue was purified by silica gel column chromatography, eluting with $\mathrm{CH}_{2} \mathrm{Cl}_{2} / \mathrm{MeOH}$ (10/1). This resulted in tert-butyl $N$-[4-[(E)-2-(4-[[5-methyl-1-(oxan-2-yl)pyrazol-3yl]amino]-6-(4-methylpiperazin-1-yl)pyrimidin-2-yl)ethenyl]bicyclo[2.2.2]octan-1-yl]carbamate (350 mg, $90 \%)$ as a yellow solid. LCMS (ESI): $[\mathrm{M}+\mathrm{H}]^{+}: 607.5$.<smiles>Cc1cc(Nc2cc(N3CCN(C)CC3)nc(/C=C/C34CCC(N)(CC3)CC4)n2)nc(Nc2cc(N3CCN(C)CC3)nc(/C=C/C34CCC(NC(=O)OC(C)(C)C)(CC3)CC4)n2)c1</smiles>

2-[(E)-2-[4-Aminobicyclo[2.2.2]octan-1-yl]ethenyl]-N-[5-methyl-1-(oxan-2-yl)pyrazol-3-yl]-6-(4methylpiperazin-1-yl)pyrimidin-4-amine (16-3): To a mixture of tert-butyl $N$-[4-[(E)-2-(4-[[5-methyl-1(oxan-2-yl)pyrazol-3-yl]amino]-6-(4-methylpiperazin-1-yl)pyrimidin-2-yl)ethenyl]bicyclo[2.2.2]octan-1yl]carbamate (330 mg, $0.544 \mathrm{mmol}, 1$ equiv) in DCM $(33 \mathrm{~mL}$ ) were added TFA $(3.3 \mathrm{~mL})$. The resulting mixture was stirred at $0{ }^{\circ} \mathrm{C}$ for 1 hour. The mixture was concentrated under vacuum. This resulted in 2- 
[(E)-2-[4-aminobicyclo[2.2.2]octan-1-yl]ethenyl]- $N$-[5-methyl-1-(oxan-2-yl)pyrazol-3-yl]-6-(4-

methylpiperazin-1-yl)pyrimidin-4-amine ( $250 \mathrm{mg}$, crude) as a yellow crude oil. LCMS (ESI): [M+H]+: 507.3.<smiles>C=CC(=O)NC12CCC(/C=C/c3nc(Nc4cc(C)n([In])n4)cc(N4CCN(C)CC4)n3)(CC1)CC2(NC(=O)C=C)C(C)(C)C</smiles>

$N$-[4-[(E)-2-(4-[[5-Methyl-1-(oxan-2-yl)pyrazol-3-yl]amino]-6-(4-methylpiperazin-1-yl)pyrimidin-2-

yl)ethenyl]bicyclo[2.2.2]octan-1-yl]prop-2-enamide (16-4): To a mixture of 2-[(E)-2-[4aminobicyclo[2.2.2]octan-1-yl]ethenyl]- $N$-[5-methyl-1-(oxan-2-yl)pyrazol-3-yl]-6-(4-methylpiperazin-1$\mathrm{yl}$ )pyrimidin-4-amine (250 mg, $0.493 \mathrm{mmol}, 1$ equiv) in DCM (15 mL) were added triethylamine (150 $\mathrm{mg}$, $1.480 \mathrm{mmol}, 3$ equiv) and acryloyl chloride ( $49 \mathrm{mg}, 0.543 \mathrm{mmol}, 1.1$ equiv) dropwise at $0{ }^{\circ} \mathrm{C}$. The resulting mixture was stirred at $0{ }^{\circ} \mathrm{C}$ for 2 hours. The reaction was quenched with $\mathrm{MeOH}$ and concentrated under vacuum. The residue was purified by silica gel column chromatography, eluting with $\mathrm{CH}_{2} \mathrm{Cl}_{2} / \mathrm{MeOH}(10 / 1)$. This resulted in $\mathrm{N}$-[4-[(E)-2-(4-[[5-methyl-1-(oxan-2-yl)pyrazol-3-yl]amino]-6-(4-methylpiperazin-1yl)pyrimidin-2-yl)ethenyl]bicyclo[2.2.2]octan-1-yl]prop-2-enamide (140 mg, 51\%) as a yellow solid. LCMS (ESI): $[\mathrm{M}+\mathrm{H}]^{+}: 561.3$.

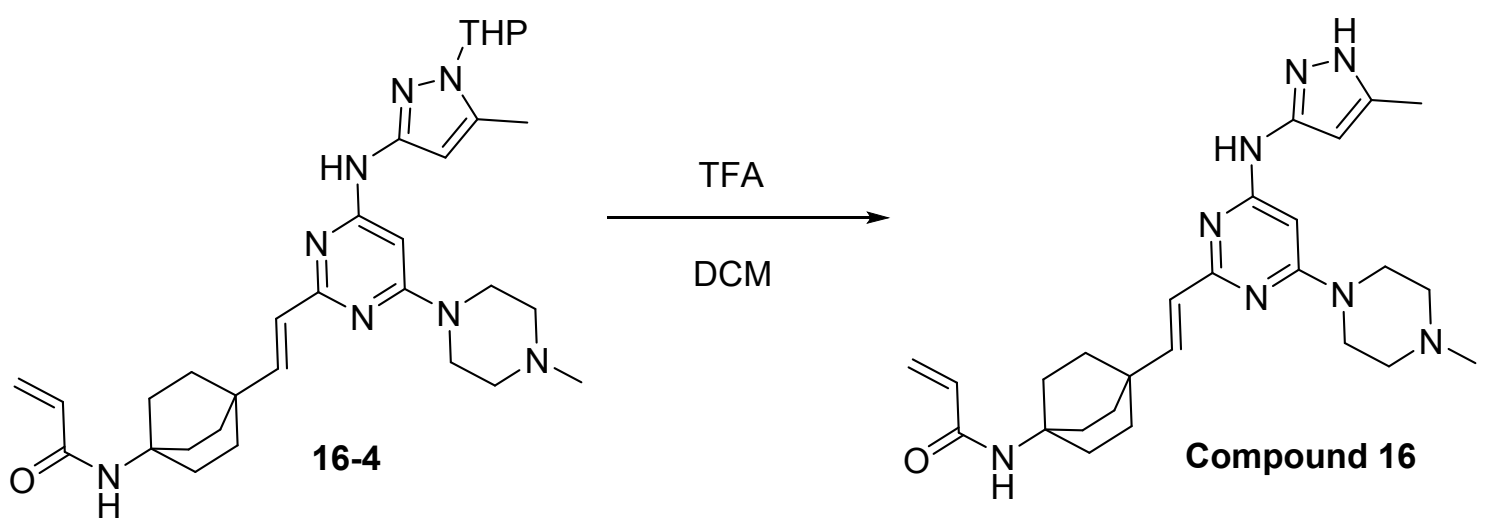

$N$-[4-[(E)-2-[4-[(5-Methyl-1H-pyrazol-3-yl)amino]-6-(4-methylpiperazin-1-yl)pyrimidin-2-

yl]ethenyl]bicyclo[2.2.2]octan-1-yl]prop-2-enamide (Compound 16): To a mixture of $\mathrm{N}$-[4-[(E)-2-(4-[[5methyl-1-(oxan-2-yl)pyrazol-3-yl]amino]-6-(4-methylpiperazin-1-yl)pyrimidin-2-

yl)ethenyl]bicyclo[2.2.2]octan-1-yl]prop-2-enamide (100 mg, 0.178 mmol, 1 equiv) in DCM (4 mL) were 
added TFA $(1 \mathrm{~mL})$. The resulting mixture was stirred at $40{ }^{\circ} \mathrm{C}$ for 1 hour. The resulting mixture was concentrated under vacuum. The crude product was purified by Prep-HPLC with the following conditions: Column: XBridge Shield RP18 OBD Column, 30*150mm, 5um; Mobile Phase A: Water (10MMOL/L $\mathrm{NH}_{4} \mathrm{HCO}_{3}$ ), Mobile Phase B: ACN; Flow rate: $60 \mathrm{~mL} / \mathrm{min}$; Gradient: $25 \mathrm{~B}$ to $55 \mathrm{~B}$ in $7 \mathrm{~min} ; 254 \mathrm{~nm}$. This resulted in $\quad \mathrm{N}$-[4-[(E)-2-[4-[(5-methyl-1H-pyrazol-3-yl)amino]-6-(4-methylpiperazin-1-yl)pyrimidin-2yl]ethenyl]bicyclo[2.2.2]octan-1-yl]prop-2-enamide (20.4 mg, 24\%) as a white solid. LCMS (ESI): [M+H] ${ }^{+}$: 477.3. ${ }^{1} \mathrm{H}$ NMR $\left(300 \mathrm{MHz}, \mathrm{CD}_{3} \mathrm{OD}\right) \delta 6.94-6.83(\mathrm{~m}, 1 \mathrm{H}), 6.31-6.05(\mathrm{~m}, 4 \mathrm{H}), 5.98-5.92(\mathrm{~m}, 1 \mathrm{H}), 5.60-$ $5.50(\mathrm{~m}, 1 \mathrm{H}), 3.66-3.57(\mathrm{~m}, 4 \mathrm{H}), 2.56-2.46(\mathrm{~m}, 4 \mathrm{H}), 2.33(\mathrm{~s}, 3 \mathrm{H}), 2.26(\mathrm{~s}, 3 \mathrm{H}), 2.07-1.96(\mathrm{~m}, 6 \mathrm{H}), 1.80-$ $1.69(\mathrm{~m}, 6 \mathrm{H})$.

\section{Compound 17:}
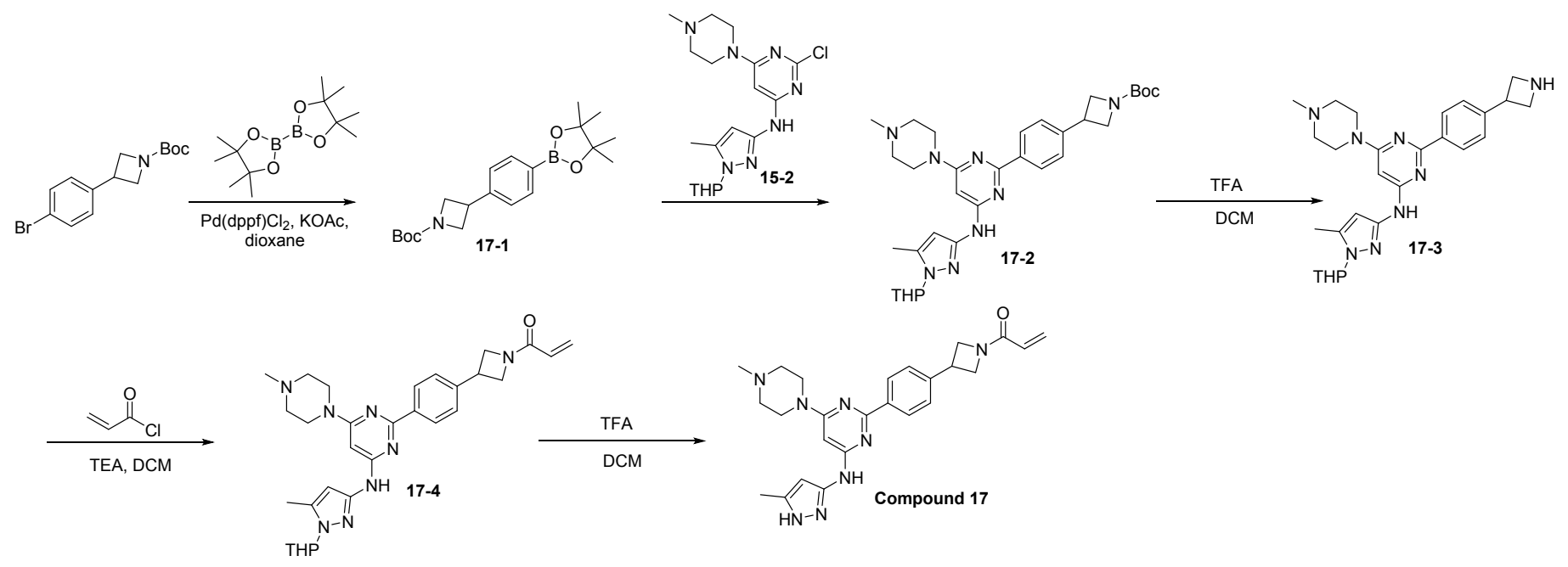<smiles>CC(C)(C)OC(=O)N1CC(c2ccc(B3OC(C)(C)C(C)(C)O3)cc2)C1</smiles>

tert-Butyl 3-[4-(4,4,5,5-tetramethyl-1,3,2-dioxaborolan-2-yl)phenyl]azetidine-1-carboxylate (17-1): To a mixture of tert-butyl 3-(4-bromophenyl)azetidine-1-carboxylate (350 mg, $1.121 \mathrm{mmol}, 1$ equiv) and bis(pinacolato)diboron (569 mg, $2.242 \mathrm{mmol}, 2$ equiv) in dioxane $(6 \mathrm{~mL}$ ) were added KOAc (330 mg, 3.363 mmol, 3 equiv) and $\mathrm{Pd}(\mathrm{dppf}) \mathrm{Cl}_{2}\left(82 \mathrm{mg}, 0.112 \mathrm{mmol}, 0.1\right.$ equiv). The resulting mixture was stirred at $80^{\circ} \mathrm{C}$ overnight under a nitrogen atmosphere. The resulting mixture was concentrated under vacuum. The residue was purified by Prep-TLC $\left(\mathrm{CH}_{2} \mathrm{Cl}_{2} / \mathrm{MeOH} 10 / 1\right)$ to afford tert-butyl 3-[4-(4,4,5,5-tetramethyl-1,3,2dioxaborolan-2-yl)phenyl]azetidine-1-carboxylate $(400 \mathrm{mg}, 74 \%)$ as a yellow oil. LCMS (ESI): [M+H] ${ }^{+}$: 360.3 . 
<smiles>CC(C)(C)OC(=O)N1CC(c2ccc(B3OC(C)(C)C(C)(C)O3)cc2)C1</smiles><smiles>Cc1cc(Nc2cc(N3CCN(C)CC3)nc(Cl)n2)nn1P</smiles><smiles>Cc1cc(Nc2cc(N3CCN(C)CC3)nc(-c3ccc(C4CN(C(=O)OC(C)(C)C)C4)cc3)n2)nn1C</smiles>

THP

tert-Butyl 3-[4-(4-[[5-methyl-1-(oxan-2-yl)pyrazol-3-yl]amino]-6-(4-methylpiperazin-1-yl)pyrimidin-2yl)phenyl]azetidine-1-carboxylate (17-2): To a mixture of tert-butyl 3-[4-(4,4,5,5-tetramethyl-1,3,2dioxaborolan-2-yl)phenyl]azetidine-1-carboxylate (200 mg, $0.557 \mathrm{mmol}, 1$ equiv) and 2-chloro- $N$-[5methyl-1-(oxan-2-yl)pyrazol-3-yl]-6-(4-methylpiperazin-1-yl)pyrimidin-4-amine (218 mg, $0.557 \mathrm{mmol}, 1$ equiv) in THF (5 mL) were added $\mathrm{K}_{3} \mathrm{PO}_{4}\left(354 \mathrm{mg}, 1.670 \mathrm{mmol}, 3\right.$ equiv) in $\mathrm{H}_{2} \mathrm{O}(1 \mathrm{~mL})$ and $\mathrm{Pd}(\mathrm{dppf}) \mathrm{Cl}_{2}(81$ $\mathrm{mg}, 0.111 \mathrm{mmol}, 0.2$ equiv). The resulting mixture was stirred at $80{ }^{\circ} \mathrm{C}$ overnight under nitrogen atmosphere. The resulting mixture was concentrated under vacuum. The residue was purified by PrepTLC $\left(\mathrm{CH}_{2} \mathrm{Cl}_{2} / \mathrm{MeOH}=10 / 1\right)$ to afford tert-butyl 3-[4-(4-[[5-methyl-1-(oxan-2-yl)pyrazol-3-yl]amino]-6-(4methylpiperazin-1-yl)pyrimidin-2-yl)phenyl]azetidine-1-carboxylate (250 mg, 53\%) as a yellow solid. LCMS (ESI): $[\mathrm{M}+\mathrm{H}]^{+}: 589.5$.

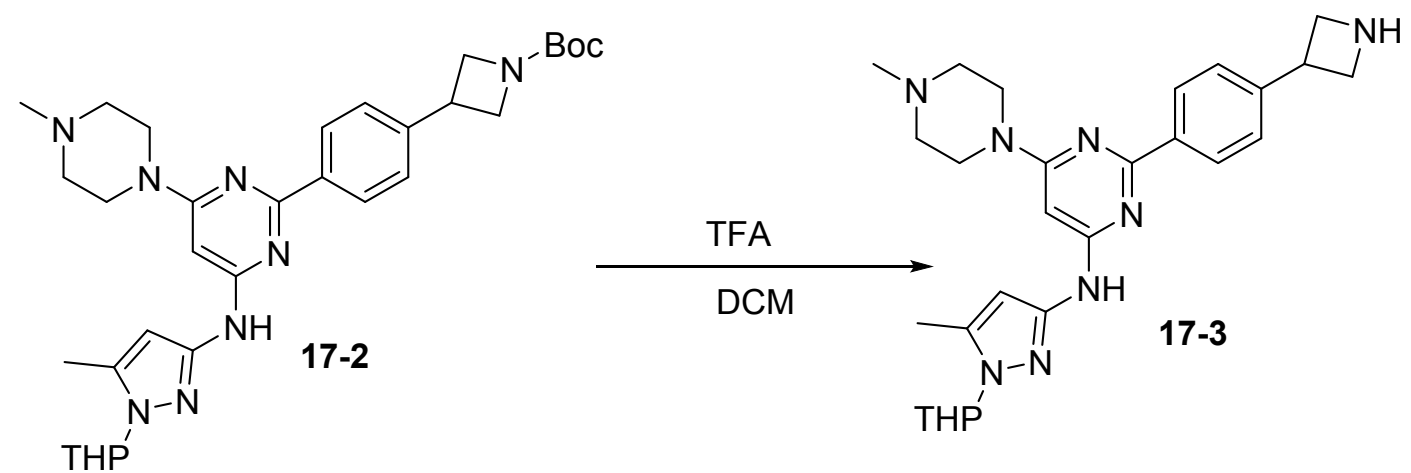

\section{2-[4-(Azetidin-3-yl)phenyl]-N-[5-methyl-1-(oxan-2-yl)pyrazol-3-yl]-6-(4-methylpiperazin-1-}

yl)pyrimidin-4-amine (17-3): To a mixture of tert-butyl 3-[4-(4-[[5-methyl-1-(oxan-2-yl)pyrazol-3yl]amino]-6-(4-methylpiperazin-1-yl)pyrimidin-2-yl)phenyl]azetidine-1-carboxylate $(230 \mathrm{mg}, 0.391 \mathrm{mmol}$, 1 equiv) in DCM $(10 \mathrm{~mL})$ was added TFA $(2.3 \mathrm{~mL})$ The resulting mixture was stirred at $0{ }^{\circ} \mathrm{C}$ for $30 \mathrm{~min}$. The resulting mixture was concentrated under vacuum. This resulted in 2-[4-(azetidin-3-yl)phenyl]- $N$-[5methyl-1-(oxan-2-yl)pyrazol-3-yl]-6-(4-methylpiperazin-1-yl)pyrimidin-4-amine (150 mg, 79\%) as a yellow crude solid. LCMS (ESI): [M+H]+: 489.3 . 


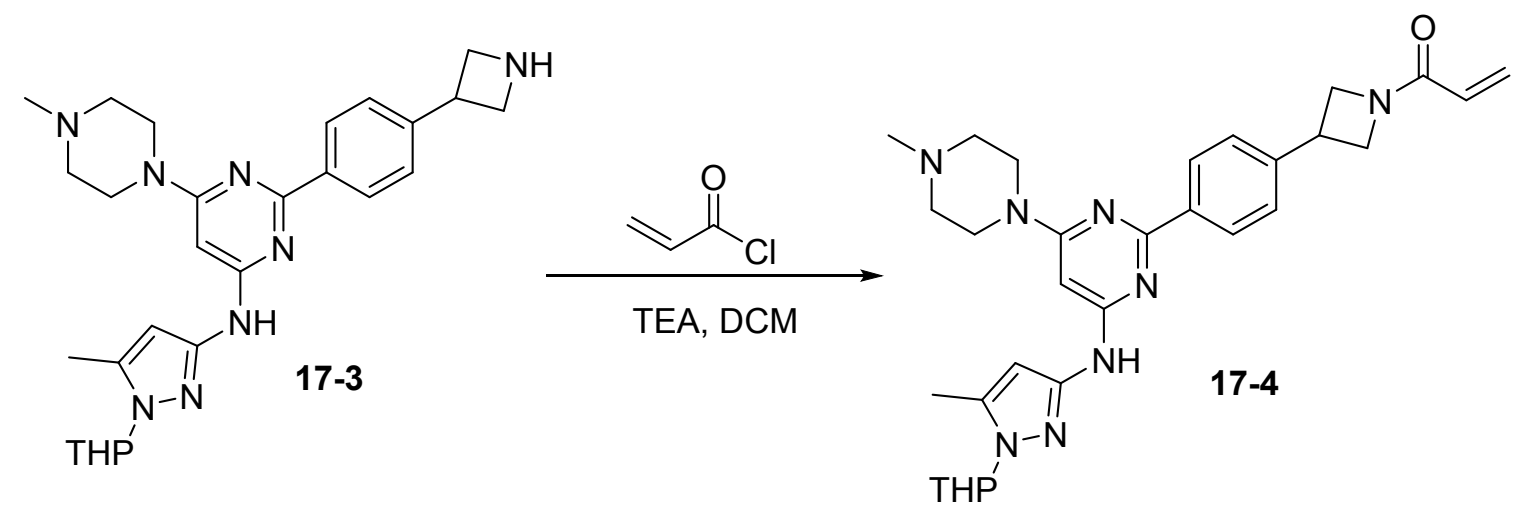

\section{1-[3-[4-(4-[[5-Methyl-1-(oxan-2-yl)pyrazol-3-yl]amino]-6-(4-methylpiperazin-1-yl)pyrimidin-2-}

yl)phenyl]azetidin-1-yl]prop-2-en-1-one (17-4): To a mixture of 2-[4-(azetidin-3-yl)phenyl]- $N$-[5-methyl1-(oxan-2-yl)pyrazol-3-yl]-6-(4-methylpiperazin-1-yl)pyrimidin-4-amine (150 mg, $0.307 \mathrm{mmol}, 1$ equiv) in DCM ( $3 \mathrm{~mL}$ ) were added triethylamine $(93 \mathrm{mg}, 0.921 \mathrm{mmol}, 3$ equiv) and acryloyl chloride $(27 \mathrm{mg}, 0.307$ mmol, 1 equiv) dropwise at $0{ }^{\circ} \mathrm{C}$. The resulting mixture was stirred at $0^{\circ} \mathrm{C}$ for 30 minutes. The reaction was quenched with $\mathrm{MeOH}$ at $0{ }^{\circ} \mathrm{C}$. The resulting mixture was concentrated under vacuum. This resulted in 1-[3-[4-(4-[[5-methyl-1-(oxan-2-yl)pyrazol-3-yl]amino]-6-(4-methylpiperazin-1-yl)pyrimidin-2yl)phenyl]azetidin-1-yl]prop-2-en-1-one (100 mg, 60\%) as a yellow crude solid. LCMS (ESI): [M+H]+: 543.3.
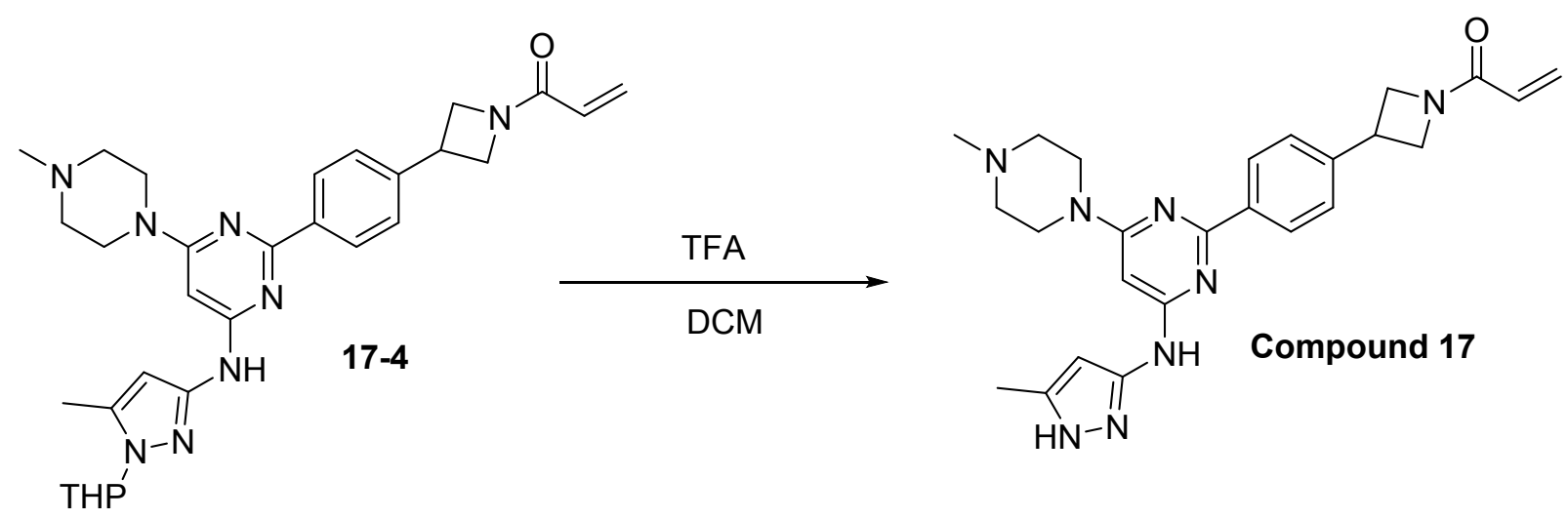

1-[3-(4-[4-[(5-Methyl-1H-pyrazol-3-yl)amino]-6-(4-methylpiperazin-1-yl)pyrimidin-2-

yl]phenyl)azetidin-1-yl]prop-2-en-1-one (Compound 17): To a mixture of 1-[3-[4-(4-[[5-methyl-1-(oxan2-yl)pyrazol-3-yl]amino]-6-(4-methylpiperazin-1-yl)pyrimidin-2-yl)phenyl]azetidin-1-yl]prop-2-en-1-one (100 mg, $0.184 \mathrm{mmol}, 1$ equiv) in DCM $(3 \mathrm{~mL})$ was added TFA $(1 \mathrm{~mL})$ dropwise at $0{ }^{\circ} \mathrm{C}$. The resulting mixture was stirred at room temperature for 30 minutes. The resulting mixture was concentrated under vacuum. The residue was purified by $\mathrm{C} 18$ eluting with $\mathrm{ACN} / \mathrm{H}_{2} \mathrm{O}(1 / 1)$ to afford 1-[3-(4-[4-[(5-methyl-1H-pyrazol-3yl)amino]-6-(4-methylpiperazin-1-yl)pyrimidin-2-yl]phenyl)azetidin-1-yl]prop-2-en-1-one (3.8 mg, 5\%) as an off-white solid. LCMS (ESI): [M+H] ${ }^{+}: 459.29 .{ }^{1} \mathrm{H}$ NMR (300 MHz, $\left.\mathrm{CD}_{3} \mathrm{OD}\right) \delta 8.41-8.32(\mathrm{~m}, 2 \mathrm{H}), 7.50-$ 
$7.42(\mathrm{~m}, 2 \mathrm{H}), 6.51-6.24(\mathrm{~m}, 3 \mathrm{H}), 6.16(\mathrm{~s}, 1 \mathrm{H}), 5.83-5.73(\mathrm{~m}, 1 \mathrm{H}), 4.82-4.70(\mathrm{~m}, 1 \mathrm{H}), 4.59-4.46(\mathrm{~m}, 1 \mathrm{H})$, $4.44-4.33(\mathrm{~m}, 1 \mathrm{H}), 4.20-4.09(\mathrm{~m}, 1 \mathrm{H}), 4.03(\mathrm{~s}, 1 \mathrm{H}), 3.73(\mathrm{~s}, 4 \mathrm{H}), 2.57(\mathrm{~s}, 4 \mathrm{H}), 2.37(\mathrm{~s}, 3 \mathrm{H}), 2.31(\mathrm{~s}, 3 \mathrm{H})$.

\section{Compound 18:}

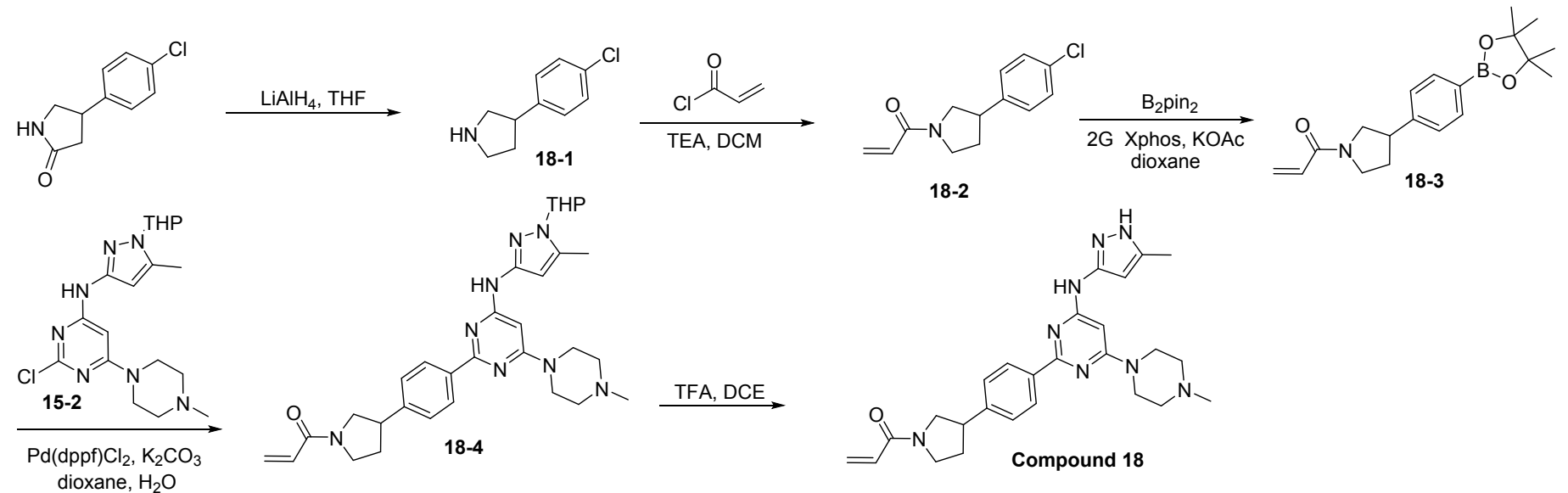<smiles>O=C1CC(c2ccc(Cl)cc2)CN1</smiles>

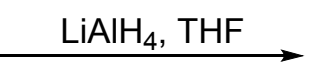<smiles>Clc1ccc(C2CCNC2)cc1</smiles>

18-1

3-(4-Chlorophenyl)pyrrolidine (18-1): To a mixture of $\mathrm{LiAlH}_{4}(2.9 \mathrm{~g}, 76.668 \mathrm{mmol}, 5$ equiv) in THF (30 mL) was added 4-(4-chlorophenyl)pyrrolidin-2-one ( $3 \mathrm{~g}, 15.334 \mathrm{mmol}, 1$ equiv). The resulting mixture was stirred at $80{ }^{\circ} \mathrm{C}$ overnight. The reaction was quenched by the addition of water at $0{ }^{\circ} \mathrm{C}$. The resulting mixture was concentrated under vacuum. The residue was purified by $\mathrm{C} 18$ reverse phase column eluting with $\mathrm{ACN} / \mathrm{H}_{2} \mathrm{O}(1 / 1)$. This resulted in $1.6 \mathrm{~g}(57 \%)$ of 3-(4-chlorophenyl)pyrrolidine as a yellow oil. LCMS (ESI): $[\mathrm{M}+\mathrm{H}]^{+}: 182.2$.<smiles>Clc1ccc(C2CCNC2)cc1</smiles>

18-1

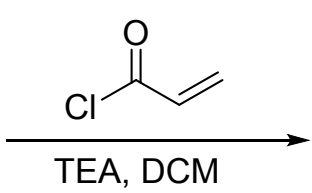

TEA, DCM<smiles>C=CC(=O)N1CCC(c2ccc(Cl)cc2)C1</smiles>

18-2

1-[3-(4-Chlorophenyl)pyrrolidin-1-yl]prop-2-en-1-one (18-2): To a mixture of 3-(4chlorophenyl)pyrrolidine ( $1.6 \mathrm{~g}, 8.808 \mathrm{mmol}, 1$ equiv) and triethylamine ( $2.67 \mathrm{~g}, 0.026 \mathrm{mmol}, 3 \mathrm{equiv})$ in 
DCM (10 mL) was added acryloyl chloride $\left(0.96 \mathrm{~g}, 0.011 \mathrm{mmol}, 1.2\right.$ equiv) at $0{ }^{\circ} \mathrm{C}$. The resulting mixture was stirred at $0{ }^{\circ} \mathrm{C}$ for 1 hour. The resulting mixture was concentrated under vacuum. The residue was applied onto a silica gel column eluting with dichloromethane/methanol (10/1). This resulted in $1.35 \mathrm{~g}$ (65\%) of 1-[3-(4-chlorophenyl)pyrrolidin-1-yl]prop-2-en-1-one as a yellow oil. LCMS (ESI): [M+H]+: 236.1.<smiles>C=CC(=O)N1CCC(c2ccc(Cl)cc2)C1</smiles>

18-2

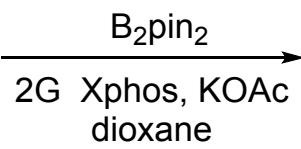

dioxane

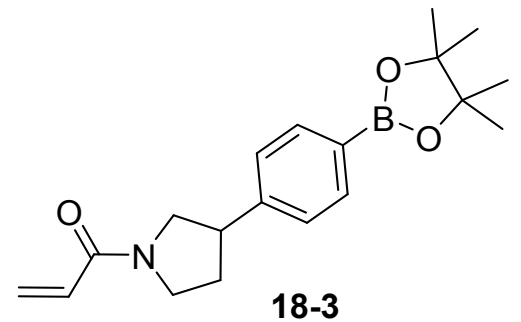

18-3

1-[3-[4-(4,4,5,5-Tetramethyl-1,3,2-dioxaborolan-2-yl)phenyl]pyrrolidin-1-yl]prop-2-en-1-one (18-3): To a mixture of 1-[3-(4-chlorophenyl)pyrrolidin-1-yl]prop-2-en-1-one (700 mg, $2.970 \mathrm{mmol}, 1$ equiv) and $\mathrm{B}_{2} \mathrm{pin}_{2}(1.5 \mathrm{~g}, 5.940 \mathrm{mmol}, 2$ equiv) in dioxane $(5 \mathrm{~mL})$ were added KOAc ( $874 \mathrm{mg}, 8.909 \mathrm{mmol}, 3$ equiv) and [2-(2-aminophenyl)phenyl](chloro)palladium; dicyclohexyl(\{2-[2,4,6-tris(propan-2yl)phenyl]phenyl\})phosphane ( $467 \mathrm{mg}, 0.594 \mathrm{mmol}, 0.2$ equiv). The resulting mixture was stirred at $70^{\circ} \mathrm{C}$ for 3 hours. The resulting mixture was concentrated under vacuum. The residue was applied onto a silica gel column eluting with PE/EtOAc (1:2). This resulted in $800 \mathrm{mg}$ (82\%) of 1-[3-[4-(4,4,5,5-tetramethyl1,3,2-dioxaborolan-2-yl)phenyl]pyrrolidin-1-yl]prop-2-en-1-one as a yellow oil. LCMS (ESI): [M+H] ${ }^{+}: 328.1$.

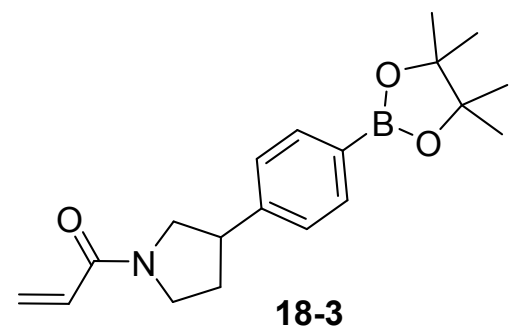

18-3
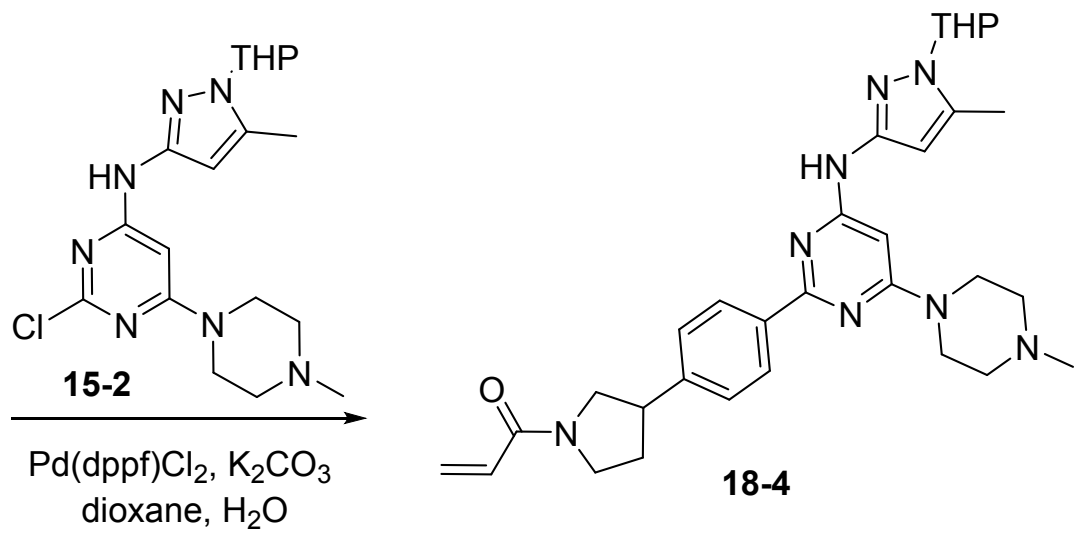

$18-4$

\section{1-[3-[4-(4-[[5-Methyl-1-(oxan-2-yl)pyrazol-3-yl]amino]-6-(4-methylpiperazin-1-yl)pyrimidin-2-}

yl)phenyl]pyrrolidin-1-yl]prop-2-en-1-one (18-4): To a mixture of 2-chloro- $N$-[5-methyl-1-(oxan-2yl)pyrazol-3-yl]-6-(4-methylpiperazin-1-yl)pyrimidin-4-amine (200 mg, $0.51 \mathrm{mmol}, 1$ equiv) and 1-[3-[4(4,4,5,5-tetramethyl-1,3,2-dioxaborolan-2-yl)phenyl]pyrrolidin-1-yl]prop-2-en-1-one $(250 \mathrm{mg}, 0.766$ mmol, 1.5 equiv) in dioxane $(5 \mathrm{~mL})$ and $\mathrm{H}_{2} \mathrm{O}(0.5 \mathrm{~mL})$ was added $\mathrm{K}_{2} \mathrm{CO}_{3}(212 \mathrm{mg}, 1.531 \mathrm{mmol}, 3$ equiv) and $\mathrm{Pd}(\mathrm{dppf}) \mathrm{Cl}_{2}$ (37 mg, $0.051 \mathrm{mmol}, 0.1$ equiv). The resulting mixture was stirred at $80{ }^{\circ} \mathrm{C}$ overnight. The resulting mixture was concentrated under vacuum. The residue was applied onto a silica gel column 
eluting with dichloromethane/methanol (10/1). This resulted in $180 \mathrm{mg}(63 \%)$ of 1-[3-[4-(4-[[5-methyl-1(oxan-2-yl)pyrazol-3-yl]amino]-6-(4-methylpiperazin-1-yl)pyrimidin-2-yl)phenyl]pyrrolidin-1-yl]prop-2en-1-one as a yellow solid. LCMS (ESI) $[\mathrm{M}+\mathrm{H}]^{+}: 557.3$.

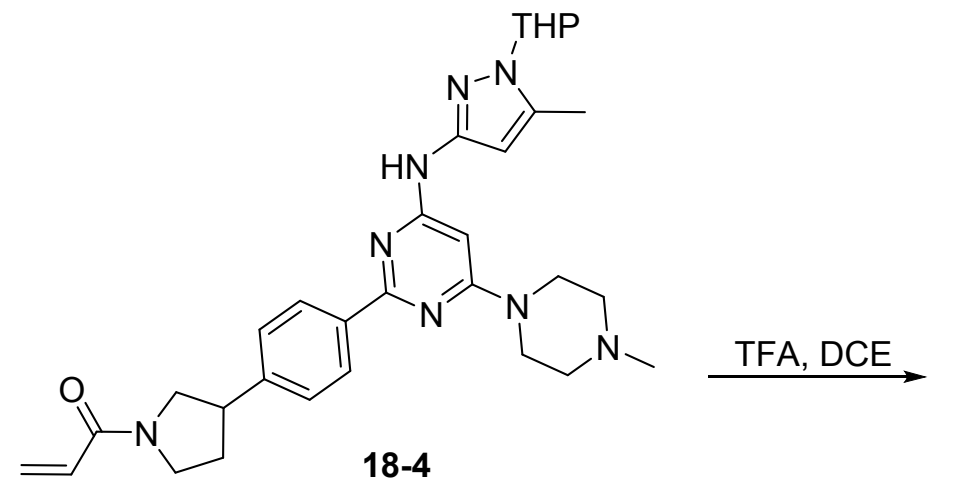<smiles>C=CC(=O)N1CCC(c2ccc(-c3nc(Nc4cc(C)[nH]n4)cc(N4CCN(C)CC4)n3)cc2)C1</smiles>

1-[3-(4-[4-[(5-Methyl-1H-pyrazol-3-yl)amino]-6-(4-methylpiperazin-1-yl)pyrimidin-2yl]phenyl)pyrrolidin-1-yl]prop-2-en-1-one (Compound 18): To a mixture of 1-[3-[4-(4-[[5-methyl-1-(oxan2-yl)pyrazol-3-yl]amino]-6-(4-methylpiperazin-1-yl)pyrimidin-2-yl)phenyl]pyrrolidin-1-yl]prop-2-en-1-one (160 mg, $0.287 \mathrm{mmol}, 1$ equiv) in DCE $(5 \mathrm{~mL}$ ) was added TFA $(2 \mathrm{~mL})$. The resulting mixture was stirred at $50{ }^{\circ} \mathrm{C}$ for 1 hour. The resulting mixture was concentrated under vacuum. The residue was purified by $\mathrm{C} 18$ reverse phase column eluting with $\mathrm{ACN} / \mathrm{H}_{2} \mathrm{O}(1 / 1)$. This resulted in $18.2 \mathrm{mg}$ (13\%) of 1-[3-(4-[4-[(5-methyl1H-pyrazol-3-yl)amino]-6-(4-methylpiperazin-1-yl)pyrimidin-2-yl]phenyl)pyrrolidin-1-yl]prop-2-en-1-one as a white solid. LCMS (ESI) [M+H] ${ }^{+}: 473.4{ }^{1} \mathrm{H} \mathrm{NMR}\left(300 \mathrm{MHz}, \mathrm{CD}_{3} \mathrm{OD}\right) \delta 8.33(\mathrm{~d}, \mathrm{~J}=8.1 \mathrm{~Hz}, 2 \mathrm{H}), 7.45-7.35$ $(\mathrm{m}, 2 \mathrm{H}), 6.75-6.59(\mathrm{~m}, 1 \mathrm{H}), 6.39-6.25(\mathrm{~m}, 2 \mathrm{H}), 6.11(\mathrm{~s}, 1 \mathrm{H}), 5.84-5.71(\mathrm{~m}, 1 \mathrm{H}), 4.19-3.97(\mathrm{~m}, 1 \mathrm{H}), 3.97$ - $3.79(\mathrm{~m}, 1 \mathrm{H}), 3.77(\mathrm{~s}, 5 \mathrm{H}), 3.60(\mathrm{~d}, J=8.1 \mathrm{~Hz}, 1 \mathrm{H}), 3.57-3.44(\mathrm{~m}, 2 \mathrm{H}), 2.67(\mathrm{~s}, 4 \mathrm{H}), 2.44(\mathrm{~d}, J=1.5 \mathrm{~Hz}$, $3 \mathrm{H}), 2.31(\mathrm{~s}, 3 \mathrm{H}), 2.29-2.09(\mathrm{~m}, 1 \mathrm{H})$.

\section{Compound 19:}



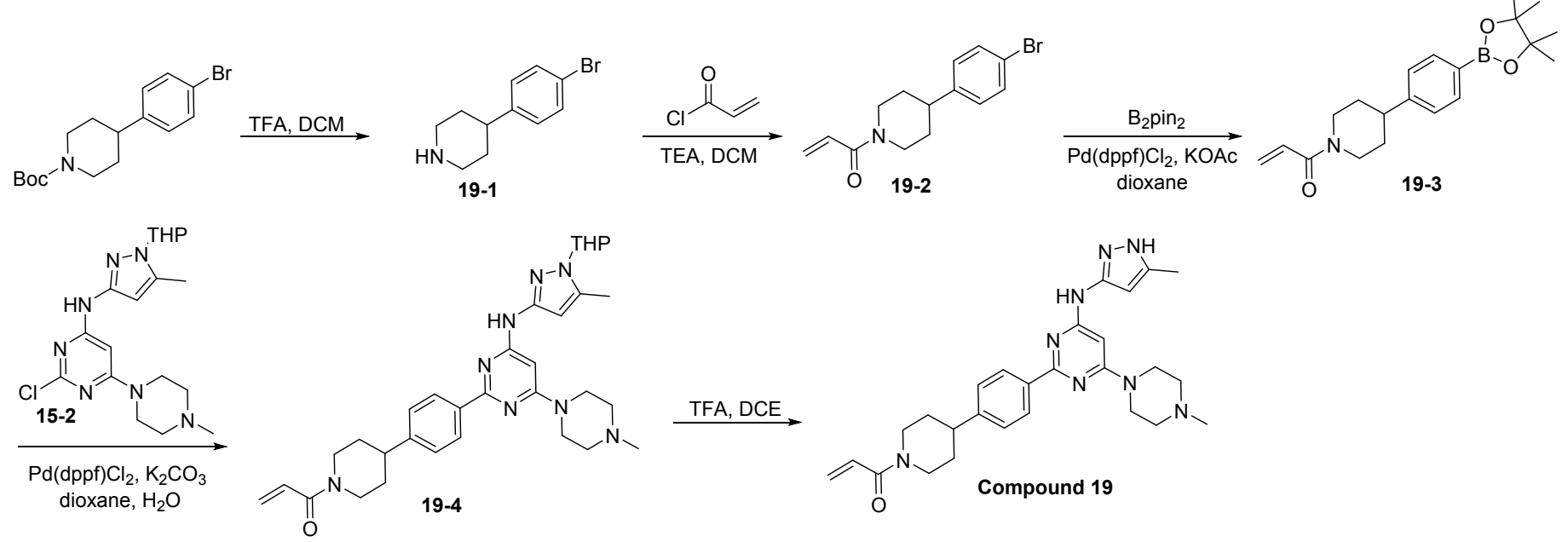<smiles>CC(C)(C)OC(=O)N1CCC(c2ccc(Br)cc2)CC1</smiles>

4-(4-Bromophenyl)piperidine (19-1): To a mixture of tert-butyl 4-(4-bromophenyl)piperidine-1carboxylate (1.5 g, $4.408 \mathrm{mmol}, 1$ equiv) in DCM $(15 \mathrm{~mL})$ was added TFA $(5 \mathrm{~mL})$. The resulting mixture was stirred at room temperature for 1 hour. The resulting mixture was concentrated under vacuum. The residue was purified by $\mathrm{C} 18$ reverse phase column eluting with $\mathrm{ACN} / \mathrm{H}_{2} \mathrm{O}(1 / 1)$. This resulted in $750 \mathrm{mg}$ (71\%) of 4-(4-bromophenyl)piperidine as a yellow solid. LCMS (ESI): [M+H]+: 240.0.<smiles>C=CC(=O)ON1CCC(c2ccc(Br)cc2)CC1</smiles>

1-[4-(4-Bromophenyl)piperidin-1-yl]prop-2-en-1-one $\quad$ (19-2): $\quad$ To a mixture of 4-(4bromophenyl)piperidine (750 mg, $3.123 \mathrm{mmol}, 1$ equiv) in DCM $(10 \mathrm{~mL})$ was added triethylamine $(948 \mathrm{mg}$, $9.369 \mathrm{mmol}, 3$ equiv) and acryloyl chloride (339 mg, $3.748 \mathrm{mmol}, 1.2$ equiv) at $0{ }^{\circ} \mathrm{C}$. The resulting mixture was stirred at $0{ }^{\circ} \mathrm{C}$ for 1 hour. The resulting mixture was concentrated under vacuum. The residue was applied onto a silica gel column eluting with dichloromethane/methanol (10/1). This resulted in $460 \mathrm{mg}$ (50\%) of 1-[4-(4-bromophenyl)piperidin-1-yl]prop-2-en-1-one as a yellow oil. LCMS (ESI): [M+H]+: 294.0. 
<smiles>C=CC(=O)N1CCC(c2ccc(Br)cc2)CC1</smiles>

19-2

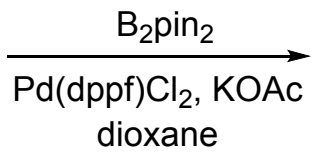<smiles>C=CC(=O)N1CCC(c2ccc(B3OC(C)(C)C(C)(C)O3)cc2)CC1</smiles>

1-[4-[4-(4,4,5,5-Tetramethyl-1,3,2-dioxaborolan-2-yl)phenyl]piperidin-1-yl]prop-2-en-1-one (19-3): To a mixture of 1-[4-(4-bromophenyl)piperidin-1-yl]prop-2-en-1-one (440 mg, $1.496 \mathrm{mmol}, 1$ equiv) in dioxane ( $5 \mathrm{~mL}$ ) was added $\mathrm{B}_{2}$ pin $_{2}$ (1.14 g, $4.487 \mathrm{mmol}, 3$ equiv), KOAc (440.3 mg, $4.487 \mathrm{mmol}, 3$ equiv) and $\mathrm{Pd}(\mathrm{dppf}) \mathrm{Cl}_{2}$ (109 mg, $0.150 \mathrm{mmol}, 0.1$ equiv). The resulting mixture was stirred at $80^{\circ} \mathrm{C}$ for 1 hour. The resulting mixture was concentrated under vacuum. The residue was applied onto a silica gel column with dichloromethane/methanol (10/1). This resulted in $480 \mathrm{mg}$ (94\%) of 1-[4-[4-(4,4,5,5-tetramethyl-1,3,2dioxaborolan-2-yl)phenyl]piperidin-1-yl]prop-2-en-1-one as a yellow solid. LCMS (ESI): [M+H]+: 342.2.
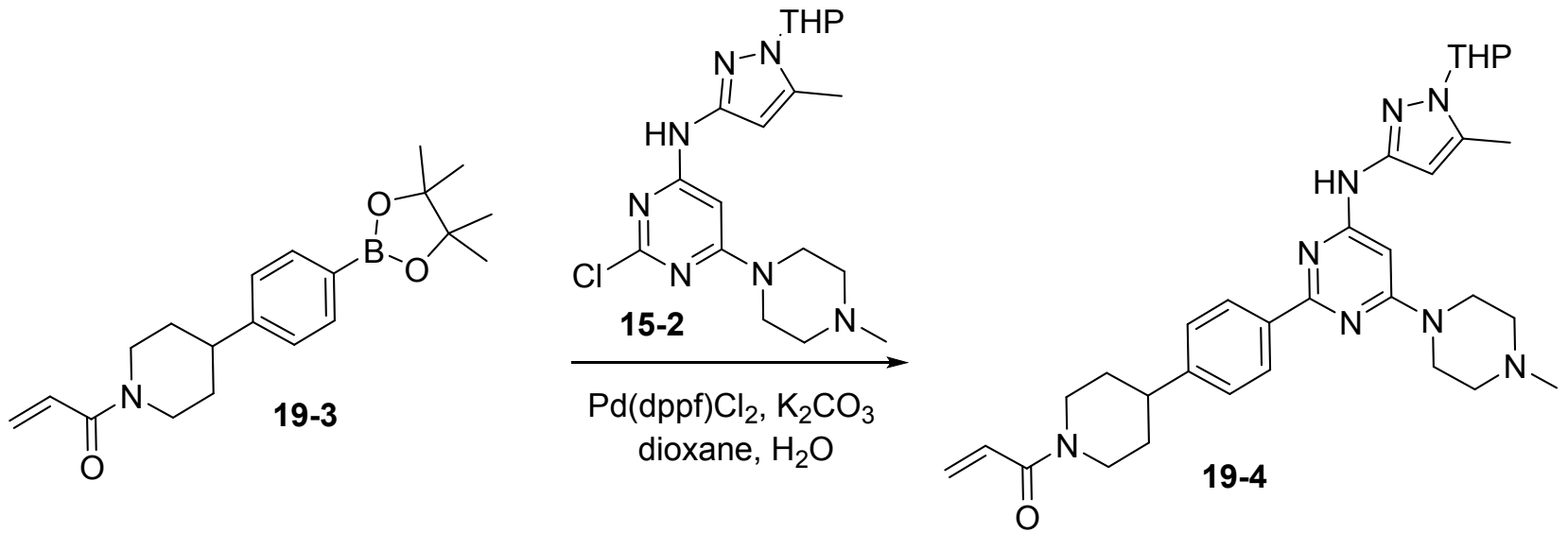

\section{1-[4-[4-(4-[[5-Methyl-1-(oxan-2-yl)pyrazol-3-yl]amino]-6-(4-methylpiperazin-1-yl)pyrimidin-2-}

yl)phenyl]piperidin-1-yl]prop-2-en-1-one (19-4): To a mixture of 2-chloro- $N$-[5-methyl-1-(oxan-2yl)pyrazol-3-yl]-6-(4-methylpiperazin-1-yl)pyrimidin-4-amine (175 mg, 0.447 mmol, 1 equiv) and 1-[4-[4(4,4,5,5-tetramethyl-1,3,2-dioxaborolan-2-yl)phenyl]piperidin-1-yl]prop-2-en-1-one (457 mg, $1.340 \mathrm{mmol}$, 3 equiv) in dioxane $\left(5 \mathrm{~mL}\right.$ ) were added $\mathrm{K}_{2} \mathrm{CO}_{3}\left(185 \mathrm{mg}, 1.340 \mathrm{mmol}, 3\right.$ equiv), $\mathrm{Pd}(\mathrm{dppf}) \mathrm{Cl}_{2}$ (33 mg, 0.045 mmol, 0.1 equiv) and $\mathrm{H}_{2} \mathrm{O}(0.5 \mathrm{~mL})$. The resulting mixture was stirred at $80{ }^{\circ} \mathrm{C}$ overnight. The resulting mixture was concentrated under vacuum. The residue was applied onto a silica gel column eluting with dichloromethane/methanol (10/1). This resulted in $110 \mathrm{mg}$ (43\%) of 1-[4-[4-(4-[[5-methyl-1-(oxan-2yl)pyrazol-3-yl]amino]-6-(4-methylpiperazin-1-yl)pyrimidin-2-yl)phenyl]piperidin-1-yl]prop-2-en-1-one as a yellow solid. LCMS (ESI) [M+H]+: 571.3 . 
<smiles>C=CC(=O)N1CCC(c2ccc(-c3nc(Nc4cc(C)n([Tl])n4)cc(N4CCN(C)CC4)n3)cc2)CC1</smiles>

\section{1-[4-(4-[4-[(5-Methyl-1H-pyrazol-3-yl)amino]-6-(4-methylpiperazin-1-yl)pyrimidin-2-}

yl]phenyl)piperidin-1-yl]prop-2-en-1-one (Compound 19): To a mixture of 1-[4-[4-(4-[[5-methyl-1-(oxan2-yl)pyrazol-3-yl]amino]-6-(4-methylpiperazin-1-yl)pyrimidin-2-yl)phenyl]piperidin-1-yl]prop-2-en-1-one (90 mg, $0.158 \mathrm{mmol}, 1$ equiv) in DCE $(3 \mathrm{~mL})$ was added TFA $(1 \mathrm{~mL})$. The resulting mixture was stirred at 50 ${ }^{\circ} \mathrm{C}$ for 1 hour. The resulting mixture was concentrated under vacuum. The crude product was purified by Prep-HPLC with the following conditions: Column, XBridge Prep OBD C18 Column, 19*250 mm, 5 um; Mobile Phase A: Water (10 mmol/L $\mathrm{NH}_{4} \mathrm{HCO}_{3}$ ), Mobile Phase B: $\mathrm{MeOH}$; Flow rate: $25 \mathrm{~mL} / \mathrm{min}$; Gradient: $54 \mathrm{~B}$ to $71 \mathrm{~B}$ in $10 \mathrm{~min} ; 254 / 220 \mathrm{~nm}$. This resulted in $16.6 \mathrm{mg}$ (21\%) of 1-[4-(4-[4-[(5-methyl-1H-pyrazol-3yl)amino]-6-(4-methylpiperazin-1-yl)pyrimidin-2-yl]phenyl)piperidin-1-yl]prop-2-en-1-one as a white solid. LCMS (ESI) $[\mathrm{M}+\mathrm{H}]^{+}:$487.3. ${ }^{1} \mathrm{H}$ NMR $\left(300 \mathrm{MHz}, \mathrm{CD}_{3} \mathrm{OD}\right) \delta 8.29(\mathrm{~d}, J=8.1 \mathrm{~Hz}, 2 \mathrm{H}), 7.33(\mathrm{~d}, J=8.1 \mathrm{~Hz}, 2 \mathrm{H})$, $6.91-6.76(\mathrm{~m}, 1 \mathrm{H}), 6.36(\mathrm{~s}, 1 \mathrm{H}), 6.29-6.17(\mathrm{~m}, 1 \mathrm{H}), 6.17(\mathrm{~s}, 1 \mathrm{H}), 5.82-5.72(\mathrm{~m}, 1 \mathrm{H}), 4.81-4.70(\mathrm{~m}, 1 \mathrm{H})$, $4.32-4.22(\mathrm{~m}, 1 \mathrm{H}), 3.77-3.68(\mathrm{~m}, 4 \mathrm{H}), 3.31-3.20(\mathrm{~m}, 1 \mathrm{H}), 2.93(\mathrm{~s}, 1 \mathrm{H}), 2.91-2.78(\mathrm{~m}, 1 \mathrm{H}), 2.61-2.52$ $(\mathrm{m}, 4 \mathrm{H}), 2.39-2.28(\mathrm{~m}, 6 \mathrm{H}), 2.02-1.92(\mathrm{~m}, 2 \mathrm{H}), 1.79-1.61(\mathrm{~m}, 2 \mathrm{H})$.

\section{Compound 20:}




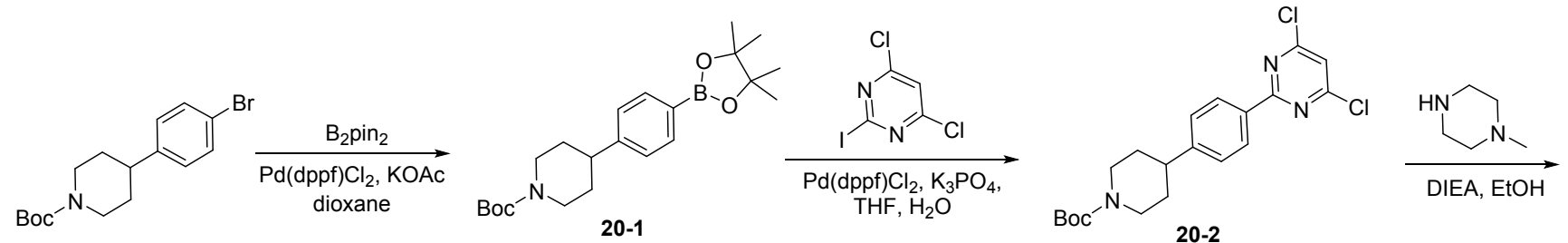<smiles>CC(C)OC(=O)C(C)(C)C</smiles>

20-1
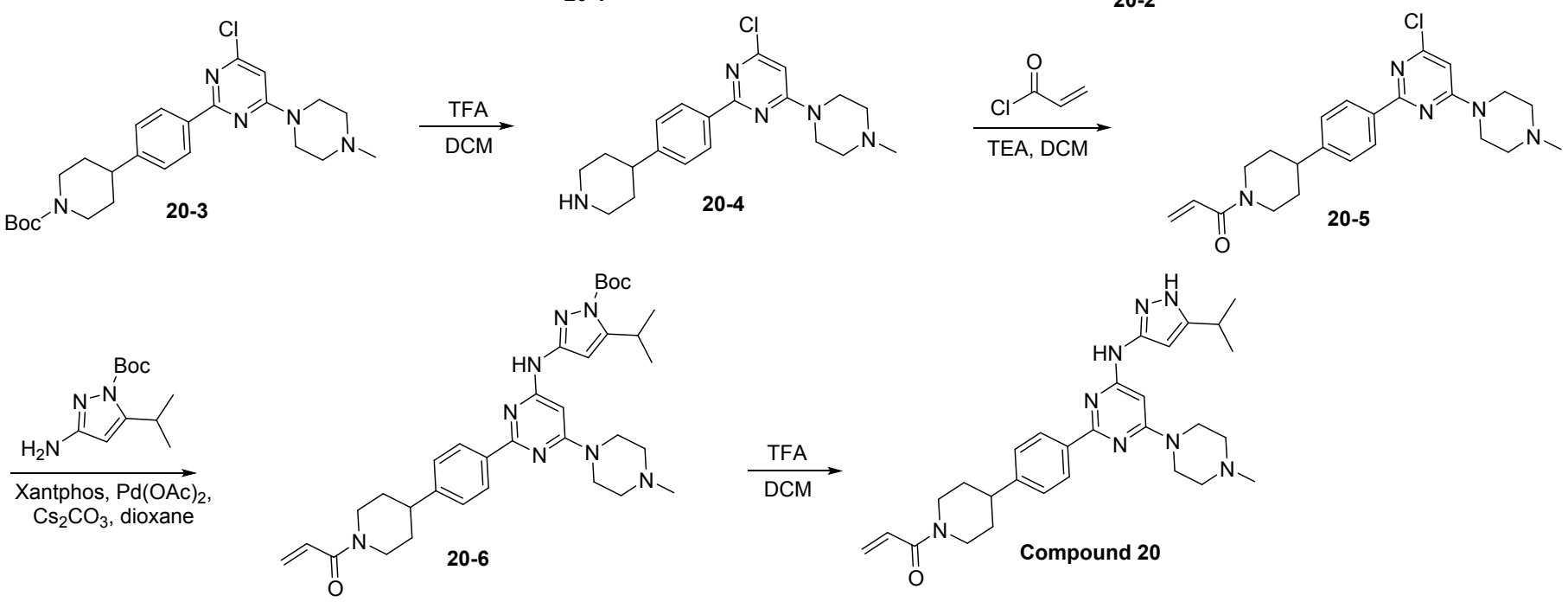

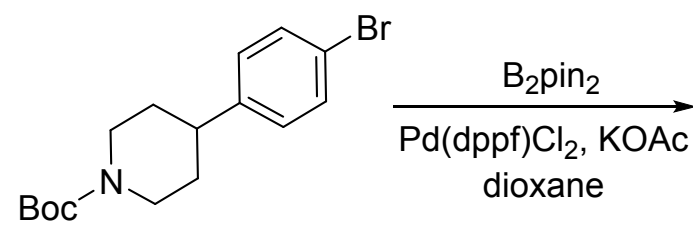<smiles>CC(C)(C)OC(=O)N1CCC(c2ccc(B3OC(C)(C)C(C)(C)O3)cc2)CC1</smiles>

\section{0-1}

tert-Butyl 4-[4-(4,4,5,5-tetramethyl-1,3,2-dioxaborolan-2-yl)phenyl]piperidine-1-carboxylate (20-1): To a mixture of tert-butyl 4-(4-bromophenyl)piperidine-1-carboxylate ( $2 \mathrm{~g}, 5.878 \mathrm{mmol}, 1$ equiv) in dioxane $(20 \mathrm{~mL})$ was added $\mathrm{B}_{2} \mathrm{pin}_{2}(2.99 \mathrm{~g}, 11.756 \mathrm{mmol}, 2$ equiv), KOAc (1.73 g, $17.634 \mathrm{mmol}, 3$ equiv) and $\mathrm{Pd}(\mathrm{dppf}) \mathrm{Cl}_{2}$ (430 mg, $0.588 \mathrm{mmol}, 0.1$ equiv). The resulting mixture was stirred at $80^{\circ} \mathrm{C}$ for 1 hour. The resulting mixture was concentrated under vacuum. The residue was applied onto a silica gel column eluting with ethyl acetate/petroleum ether (1/2). This resulted in $2 \mathrm{~g}(88 \%)$ tert-butyl 4-[4-(4,4,5,5tetramethyl-1,3,2-dioxaborolan-2-yl)phenyl]piperidine-1-carboxylate as a yellow solid. LCMS (ESI): $[\mathrm{M}+\mathrm{H}]^{+}: 388.3$. 
<smiles>CC(C)(C)OC(=O)N1CCC(c2ccc(B3OC(C)(C)C(C)(C)O3)cc2)CC1</smiles>

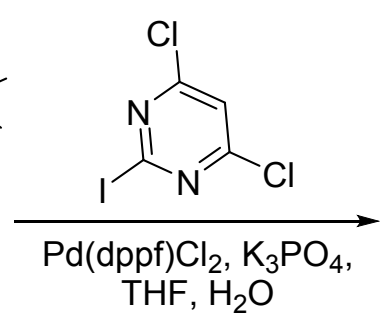<smiles>CC(C)(C)OC(=O)N1CCC(c2ccc(-c3nc(Cl)cc(Cl)n3)cc2)CC1</smiles>

tert-Butyl 4-[4-(4,6-dichloropyrimidin-2-yl)phenyl]piperidine-1-carboxylate (20-2): To a mixture of tertbutyl 4-[4-(4,4,5,5-tetramethyl-1,3,2-dioxaborolan-2-yl)phenyl]piperidine-1-carboxylate (2 g, $5.164 \mathrm{mmol}$, 1 equiv) and 4,6-dichloro-2-iodopyrimidine (1.42 g, $5.164 \mathrm{mmol}, 1$ equiv) in THF (20 mL) were added $\mathrm{Pd}$ (dppf) $\mathrm{Cl}_{2}$ (378 mg, $0.516 \mathrm{mmol}, 0.1$ equiv) and $\mathrm{K}_{3} \mathrm{PO}_{4}\left(3.29 \mathrm{mg}, 15.491 \mathrm{mmol}, 3\right.$ equiv) in $\mathrm{H}_{2} \mathrm{O}(2 \mathrm{~mL})$. The resulting mixture was stirred at $40{ }^{\circ} \mathrm{C}$ for 2 hours. The residue was applied onto a silica gel column eluting with ethyl acetate/petroleum ether (1/2). This resulted in $1.2 \mathrm{~g}$ (57\%) of tert-butyl 4-[4-(4,6dichloropyrimidin-2-yl)phenyl]piperidine-1-carboxylate as a yellow solid. LCMS (ESI): [M+H]+: 408.1 .<smiles>CC(C)(C)OC(=O)N1CCC(c2ccc(-c3nc(Cl)cc(Cl)n3)cc2)CC1</smiles>

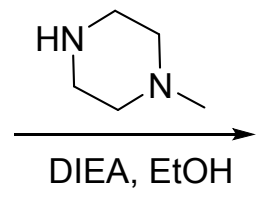<smiles>CN1CCN(c2cc(Cl)nc(-c3ccc(C4CCN(C(=O)OC(C)(C)C)CC4)cc3)n2)CC1</smiles>

tert-Butyl 4-[4-[4-chloro-6-(4-methylpiperazin-1-yl)pyrimidin-2-yl]phenyl]piperidine-1-carboxylate (203): To a mixture of tert-butyl 4-[4-(4,6-dichloropyrimidin-2-yl)phenyl]piperidine-1-carboxylate $600 \mathrm{mg}$, $1.469 \mathrm{mmol}, 1$ equiv) in EtOH (10 mL) was added 1-methylpiperazine (147 mg, $1.469 \mathrm{mmol}, 1$ equiv) and diisopropylethylamine (569 $\mathrm{mg}, 4.408 \mathrm{mmol}, 3$ equiv). The resulting mixture was stirred at room temperature for 2 hours. The resulting mixture was concentrated under vacuum. The residue was applied onto a silica gel column eluting with dichloromethane/methanol (20/1). This resulted in $560 \mathrm{mg}(81 \%)$ of tert-butyl 4-[4-[4-chloro-6-(4-methylpiperazin-1-yl)pyrimidin-2-yl]phenyl]piperidine-1-carboxylate as a yellow solid. LCMS (ESI): [M+H]+: 472.2.<smiles>CN1CCN(c2cc(Cl)nc(-c3ccc(C4CCN(C(=O)C(C)(C)C(C)(C)C)CC4)cc3)n2)CC1</smiles><smiles>CN1CCN(c2cc(Cl)nc(-c3ccc(C4CCNCC4)cc3)n2)CC1</smiles> 
4-Chloro-6-(4-methylpiperazin-1-yl)-2-[4-(piperidin-4-yl)phenyl]pyrimidine (20-4): To a mixture of tertbutyl 4-[4-[4-chloro-6-(4-methylpiperazin-1-yl)pyrimidin-2-yl]phenyl]piperidine-1-carboxylate (560 mg, $1.186 \mathrm{mmol}, 1$ equiv) in DCM (5 mL) was added TFA $(2 \mathrm{~mL})$. The resulting mixture was stirred at room temperature for 1 hour. The resulting mixture was concentrated under vacuum. This resulted in $510 \mathrm{mg}$ (crude) of 4-chloro-6-(4-methylpiperazin-1-yl)-2-[4-(piperidin-4-yl)phenyl]pyrimidine as a yellow solid. LCMS (ESI): $[\mathrm{M}+\mathrm{H}]^{+}: 372.2$.<smiles>CN1CCN(c2cc(Cl)nc(-c3ccc(C4CCNCC4)cc3)n2)CC1</smiles><smiles>C=CC(=O)Cl</smiles><smiles>C=CC(=O)N1CCC(c2ccc(-c3nc(Cl)cc(N4CCN(C)CC4)n3)cc2)CC1</smiles>

1-(4-[4-[4-Chloro-6-(4-methylpiperazin-1-yl)pyrimidin-2-yl]phenyl]piperidin-1-yl)prop-2-en-1-one (205): To a mixture of 4-chloro-6-(4-methylpiperazin-1-yl)-2-[4-(piperidin-4-yl)phenyl]pyrimidine (230 mg, $0.618 \mathrm{mmol}, 1$ equiv) in DCM (10 mL) was added triethylamine (188 mg, $1.855 \mathrm{mmol}, 3$ equiv) and acryloyl chloride ( $67 \mathrm{mg}, 0.742 \mathrm{mmol}, 1.2$ equiv). The resulting mixture was stirred at $0{ }^{\circ} \mathrm{C}$ for 1 hour. The reaction was quenched with $\mathrm{MeOH}$. The resulting mixture was concentrated under vacuum. The residue was applied onto a silica gel column eluting with dichloromethane/methanol (20/1). This resulted in $180 \mathrm{mg}$ (68\%) of 1-(4-[4-[4-chloro-6-(4-methylpiperazin-1-yl)pyrimidin-2-yl]phenyl]piperidin-1-yl)prop-2-en-1one as a yellow solid. LCMS (ESI) [M+H]+: 426.2 .<smiles>C=CC(=O)N1CCC(c2ccc(-c3nc(Cl)cc(N4CCN(C)CC4)n3)cc2)CC1</smiles>

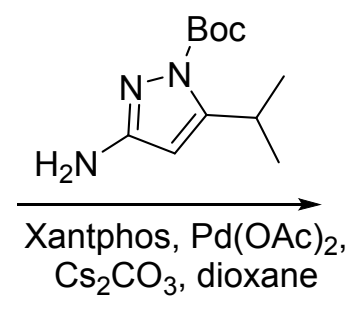<smiles>C=CC(=O)N1CCC(c2ccc(-c3nc(Nc4cc(C(C)C)n(C(=O)OC(C)(C)C)n4)cc(N4CCN(C)CC4)n3)cc2)CC1</smiles>

tert-Butyl 5-isopropyl-3-[[6-(4-methylpiperazin-1-yl)-2-[4-[1-(prop-2-enoyl)piperidin-4yl]phenyl]pyrimidin-4-yl]amino]pyrazole-1-carboxylate (20-6): To a mixture of 1-(4-[4-[4-chloro-6-(4methylpiperazin-1-yl)pyrimidin-2-yl]phenyl]piperidin-1-yl)prop-2-en-1-one (160 mg, $0.376 \mathrm{mmol}, 1$ equiv) and tert-butyl 3-amino-5-isopropylpyrazole-1-carboxylate ( $85 \mathrm{mg}, 0.376 \mathrm{mmol}, 1$ equiv) in dioxane (5 mL) 
were added $\mathrm{Cs}_{2} \mathrm{CO}_{3}$ (159 mg, $0.488 \mathrm{mmol}, 1.3$ equiv), Xantphos (43 mg, $0.075 \mathrm{mmol}, 0.2$ equiv) and $\mathrm{Pd}(\mathrm{OAc})_{2}\left(8 \mathrm{mg}, 0.038 \mathrm{mmol}, 0.1\right.$ equiv). The resulting mixture was stirred at $80^{\circ} \mathrm{C}$ for 1 hour. The resulting mixture was concentrated under vacuum. The residue was applied onto a silica gel column eluting with dichloromethane/methanol (15/1). This resulted in $130 \mathrm{mg}$ (56\%) of tert-butyl 5-isopropyl-3-[[6-(4methylpiperazin-1-yl)-2-[4-[1-(prop-2-enoyl)piperidin-4-yl]phenyl]pyrimidin-4-yl]amino]pyrazole-1carboxylate as a yellow solid. LCMS (ESI): [M+H] $]^{+}$: 615.4.
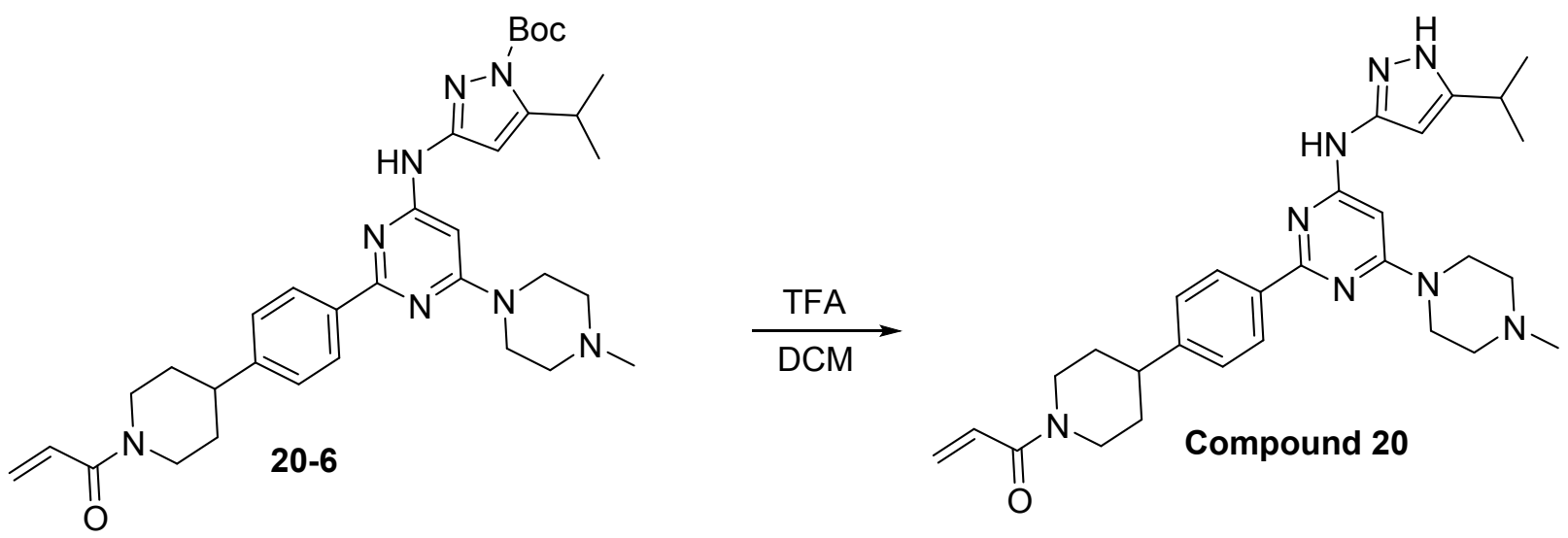

1-[4-(4-[4-[(5-Isopropyl-1H-pyrazol-3-yl)amino]-6-(4-methylpiperazin-1-yl)pyrimidin-2-

yl]phenyl)piperidin-1-yl]prop-2-en-1-one (Compound 20): To a mixture of tert-butyl 5-isopropyl-3-[[6-(4methylpiperazin-1-yl)-2-[4-[1-(prop-2-enoyl)piperidin-4-yl]phenyl]pyrimidin-4-yl]amino]pyrazole-1carboxylate (110 mg, $0.179 \mathrm{mmol}, 1$ equiv) in DCM $(3 \mathrm{~mL})$ was added TFA (1 mL). The resulting mixture was stirred at room temperature for 30 minutes. The resulting mixture was concentrated under vacuum. The crude product was purified by Prep-HPLC with the following conditions: Column, XBridge Shield RP18 OBD Column, 30*150 mm, 5 um; Mobile Phase A: Water (10 mmoL/L NH${ }_{4} \mathrm{HCO}_{3}$ ), Mobile Phase B: ACN; Flow rate: $60 \mathrm{~mL} / \mathrm{min}$; Gradient: $34 \mathrm{~B}$ to $59 \mathrm{~B}$ in $8 \mathrm{~min} ; 220 \mathrm{~nm}$. Product was obtained. This resulted in $23.8 \mathrm{mg}$ (26\%) of 1-[4-(4-[4-[(5-isopropyl-1H-pyrazol-3-yl)amino]-6-(4-methylpiperazin-1-yl)pyrimidin-2yl]phenyl)piperidin-1-yl]prop-2-en-1-one as a white solid. LCMS (ESI) [M+H] ${ }^{+}: 515.5 .{ }^{1} \mathrm{H}$ NMR $(300 \mathrm{MHz}$, $\left.\mathrm{CD}_{3} \mathrm{OD}\right) \delta 8.34-8.25(\mathrm{~m}, 2 \mathrm{H}), 7.38-7.29(\mathrm{~m}, 2 \mathrm{H}), 6.92-6.77(\mathrm{~m}, 1 \mathrm{H}), 6.34(\mathrm{~s}, 1 \mathrm{H}), 6.30-6.17(\mathrm{~m}, 1 \mathrm{H})$, $6.14(\mathrm{~s}, 1 \mathrm{H}), 5.83-5.72(\mathrm{~m}, 1 \mathrm{H}), 4.81-4.71(\mathrm{~m}, 1 \mathrm{H}), 4.33-4.23(\mathrm{~m}, 1 \mathrm{H}), 3.75(\mathrm{~s}, 4 \mathrm{H}), 3.32-3.21(\mathrm{~m}, 1 \mathrm{H})$, $3.10-2.75(\mathrm{~m}, 3 \mathrm{H}), 2.67-2.58(\mathrm{~m}, 4 \mathrm{H}), 2.41(\mathrm{~s}, 3 \mathrm{H}), 2.03-1.93(\mathrm{~m}, 2 \mathrm{H}), 1.72(\mathrm{~s}, 2 \mathrm{H}), 1.33(\mathrm{~d}, J=6.9 \mathrm{~Hz}$, $6 \mathrm{H})$.

\section{Compound 21:}


<smiles>Nc1cc(C2COC2)[nH]n1</smiles><smiles>[Z17][Z17]c1cc(C2COC2)nn1C(=O)OCc1ccccc1</smiles>

21-1

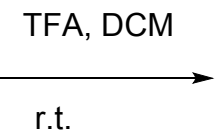<smiles>[R12]Cc1ccc(-c2nc(Nc3cc(C4COC4)n(C(=O)OC(C)(C)C)n3)cc(N3CCN(C)CC3)n2)cc1</smiles><smiles>Nc1cc(C2COC2)[nH]n1</smiles>

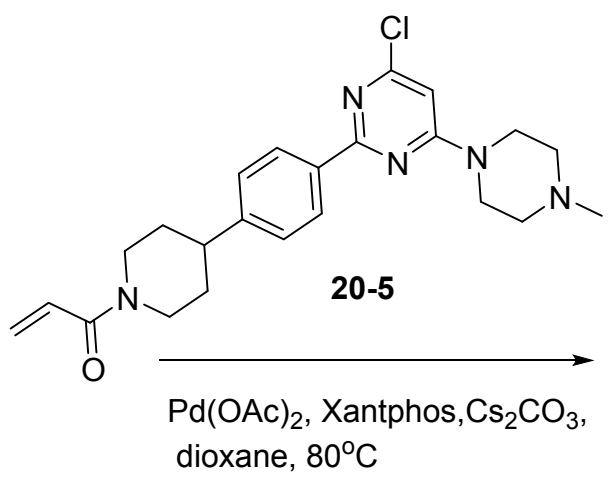

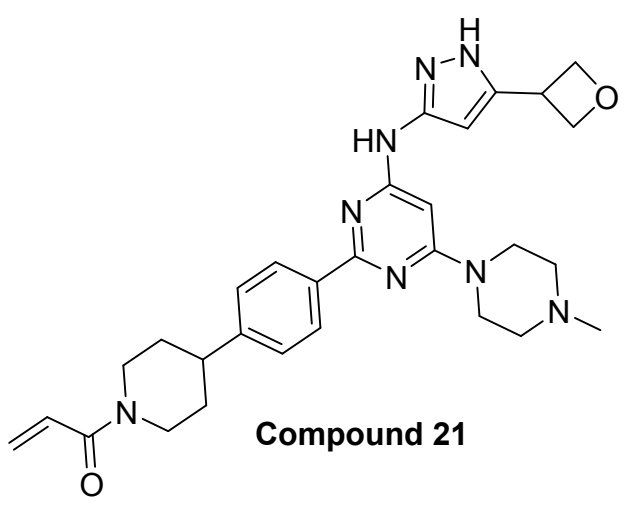<smiles>[Z17][Z17](=O)OC(=O)n1nc(N)cc1C1COC1</smiles>

tert-Butyl 3-amino-5-(oxetan-3-yl)pyrazole-1-carboxylate (21-1): To a mixture of 5-(oxetan-3-yl)-1Hpyrazol-3-amine (400 mg, $2.874 \mathrm{mmol}, 1$ equiv) in DCM $(50 \mathrm{~mL}$ ) were added $\mathrm{KOH}$ (1291 mg, $22.995 \mathrm{mmol}$, 8 equiv) in $\mathrm{H}_{2} \mathrm{O}(6 \mathrm{~mL})$ and $\mathrm{Boc}_{2} \mathrm{O}\left(753 \mathrm{mg}, 3.449 \mathrm{mmol}, 1.2\right.$ equiv) at $0{ }^{\circ} \mathrm{C}$. The resulting mixture was stirred at room temperature overnight. The combined organic layers was extracted with EtOAc $(20 \mathrm{~mL})$, dried over anhydrous $\mathrm{Na}_{2} \mathrm{SO}_{4}$. After filtration, the filtrate was concentrated under reduced pressure. The residue was purified by Prep-TLC $\left(\mathrm{CH}_{2} \mathrm{Cl}_{2} / \mathrm{MeOH}=10 / 1\right)$. This resulted in $150 \mathrm{mg}$ (19\%) of tert-butyl 3-amino-5(oxetan-3-yl)pyrazole-1-carboxylate as a white solid. LCMS (ESI): [M+H]+: 240.0. 
<smiles>[R12]CC(C)(C)OC(=O)N1CCC(c2ccc(-c3nc(Nc4cc(C5COC5)n(N5CCN(C)CC5)c4)cc(N4CCN(C)CC4)n3)cc2)CC1</smiles>

tert-Butyl 3-[[6-(4-methylpiperazin-1-yl)-2-[4-[1-(prop-2-enoyl)piperidin-4-yl]phenyl]pyrimidin-4yl]amino]-5-(oxetan-3-yl)pyrazole-1-carboxylate (21-2): To a mixture of 1-(4-[4-[4-chloro-6-(4methylpiperazin-1-yl)pyrimidin-2-yl]phenyl]piperidin-1-yl)prop-2-en-1-one (110 mg, $0.258 \mathrm{mmol}, 1$ equiv) and tert-butyl 3-amino-5-(oxetan-3-yl)pyrazole-1-carboxylate (62 mg, $0.258 \mathrm{mmol}, 1$ equiv) in dioxane (5 $\mathrm{mL}$ ) were added $\mathrm{Cs}_{2} \mathrm{CO}_{3}$ (110 mg, $0.336 \mathrm{mmol}, 1.3$ equiv), Xantphos (30 mg, $0.052 \mathrm{mmol}, 0.2$ equiv) and $\mathrm{Pd}(\mathrm{OAc})_{2}\left(6 \mathrm{mg}, 0.026 \mathrm{mmol}, 0.1\right.$ equiv). The resulting mixture was stirred at $80^{\circ} \mathrm{C}$ overnight under a hydrogen atmosphere. The resulting mixture was concentrated under vacuum. The residue was purified by Prep-TLC $\left(\mathrm{CH}_{2} \mathrm{Cl}_{2} / \mathrm{MeOH}=10 / 1\right)$. This resulted in $40 \mathrm{mg}$ (25\%) of tert-butyl 3-[[6-(4-methylpiperazin-1yl)-2-[4-[1-(prop-2-enoyl)piperidin-4-yl]phenyl]pyrimidin-4-yl]amino]-5-(oxetan-3-yl)pyrazole-1carboxylate as a yellow solid. LCMS (ESI): $[\mathrm{M}+\mathrm{H}]^{+}: 629.3$.<smiles>[R12]CC(=O)OC(=O)c1ccc(C2CCN(C(=O)C=C)CC2)cc1-c1ccc(-c2nc(Nc3cc(C4COC4)[nH]n3)cc(N3CCN(C)CC3)n2)cc1</smiles>

1-(4-[4-[4-(4-Methylpiperazin-1-yl)-6-[[5-(oxetan-3-yl)-1H-pyrazol-3-yl]amino]pyrimidin-2yl]phenyl]piperidin-1-yl)prop-2-en-1-one (Compound 21): To a mixture of tert-butyl 3-[[6-(4methylpiperazin-1-yl)-2-[4-[1-(prop-2-enoyl)piperidin-4-yl]phenyl]pyrimidin-4-yl]amino]-5-(oxetan-3yl)pyrazole-1-carboxylate ( $40 \mathrm{mg}, 0.064 \mathrm{mmol}, 1$ equiv) in DCM $(5 \mathrm{~mL})$ were added TFA $(1 \mathrm{~mL})$ dropwise at $0{ }^{\circ} \mathrm{C}$. The resulting mixture was stirred for 1 hour at room temperature. The resulting mixture was concentrated under vacuum. The crude product was purified by Prep-HPLC with the following conditions: Column: XBridge Shield RP18 OBD Column, 30*150mm,5um ; Mobile Phase A:Water(10mmol/L NH$\left.{ }_{4} \mathrm{HCO}_{3}\right)$, 
Mobile Phase B:ACN; Flow rate: $60 \mathrm{~mL} / \mathrm{min}$; Gradient:24 B to 48 B in 8 min; Detector, $254 \mathrm{~nm}$; This resulted in $\quad 3.4 \mathrm{mg} \quad(10 \%)$ of 1-(4-[4-[4-(4-methylpiperazin-1-yl)-6-[[5-(oxetan-3-yl)-1H-pyrazol-3yl]amino]pyrimidin-2-yl]phenyl]piperidin-1-yl)prop-2-en-1-one as a white solid. LCMS (ESI): [M+H]+: 529.2. ${ }^{1} \mathrm{H}$ NMR $\left(300 \mathrm{MHz}, \mathrm{CD}_{3} \mathrm{OD}\right) \delta 8.29(\mathrm{~d}, J=8.1 \mathrm{~Hz}, 2 \mathrm{H}), 7.35(\mathrm{~d}, J=8.1 \mathrm{~Hz}, 2 \mathrm{H}), 6.92-6.77(\mathrm{~m}, 1 \mathrm{H}), 6.30-$ $6.18(\mathrm{~m}, 1 \mathrm{H}), 5.83-5.72(\mathrm{~m}, 1 \mathrm{H}), 5.12-5.01(\mathrm{~m}, 2 \mathrm{H}), 4.89-4.71(\mathrm{~m}, 3 \mathrm{H}), 4.40-4.34(\mathrm{~m}, 1 \mathrm{H}), 4.33-4.23$ $(\mathrm{m}, 1 \mathrm{H}), 3.77-3.71(\mathrm{~m}, 4 \mathrm{H}), 3.32-3.21(\mathrm{~m}, 1 \mathrm{H}), 3.02-2.79(\mathrm{~m}, 2 \mathrm{H}), 2.62-2.53(\mathrm{~m}, 4 \mathrm{H}), 2.37(\mathrm{~s}, 3 \mathrm{H}), 2.03$ $-1.93(\mathrm{~m}, 3 \mathrm{H}), 1.80-1.66(\mathrm{~m}, 1 \mathrm{H}), 1.74-1.68(\mathrm{~m}, 2 \mathrm{H})$.

\section{Compound 22:}

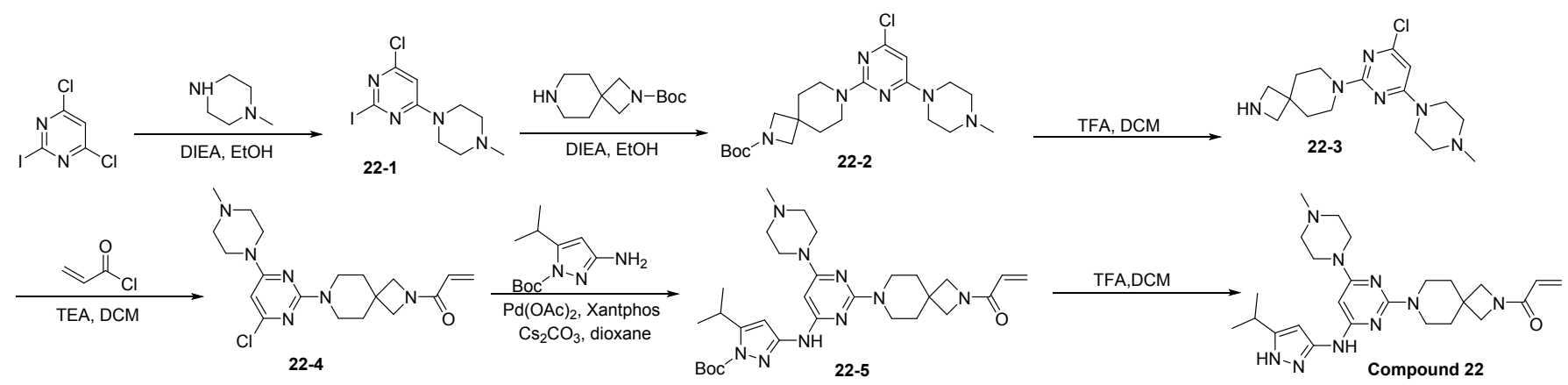<smiles>[Z10]CCN1CCNCC1CC(C)O</smiles>

4-Chloro-2-iodo-6-(4-methylpiperazin-1-yl)pyrimidine (22-1): To a mixture of 4,6-dichloro-2iodopyrimidine ( $8 \mathrm{~g}, 29.105 \mathrm{mmol}, 1$ equiv) in $\mathrm{EtOH}(100 \mathrm{~mL})$ were added diisopropylethylamine $(11.28 \mathrm{~g}$, $87.277 \mathrm{mmol}, 3$ equiv) and 1-methylpiperazine (2.92 g, $29.105 \mathrm{mmol}, 1$ equiv) dropwise at $0{ }^{\circ} \mathrm{C}$. The resulting mixture was stirred at room temperature for 1 hour. The resulting mixture was concentrated under vacuum. The residue was purified by $\mathrm{C} 18$ eluting with $\mathrm{ACN} / \mathrm{H}_{2} \mathrm{O}(1 / 1)$ to afford 4-chloro-2-iodo-6(4-methylpiperazin-1-yl)pyrimidine (8 g, 81\%) as a white solid. LCMS (ESI): [M+H] $]^{+}: 339.0$.

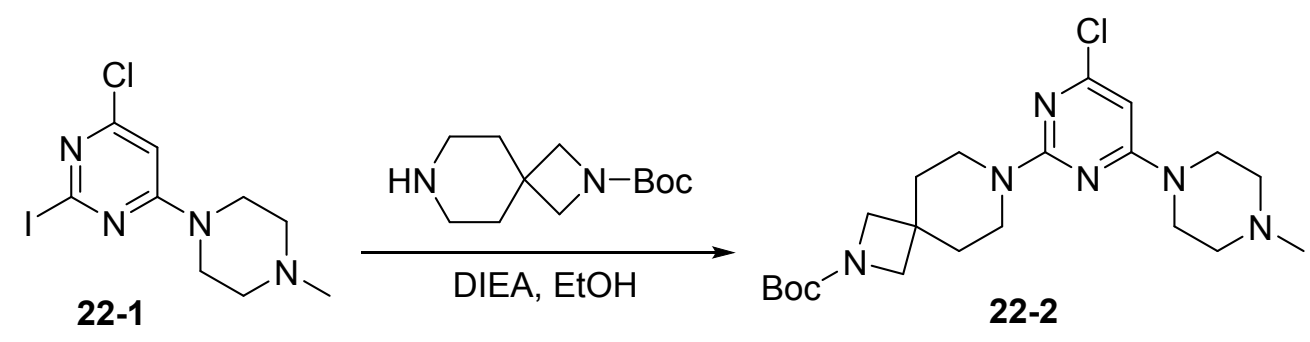



mmol, 1 equiv) and 4-chloro-2-iodo-6-(4-methylpiperazin-1-yl)pyrimidine (403 mg, $1.193 \mathrm{mmol}, 1$ equiv) in EtOH ( $6 \mathrm{~mL}$ ) was added diisopropylethylamine ( $462 \mathrm{mg}, 3.579 \mathrm{mmol}, 3$ equiv). The resulting mixture was stirred at $50{ }^{\circ} \mathrm{C}$ overnight. The resulting mixture was concentrated under vacuum. The residue was purified by silica gel column chromatography, eluting with PE/EtOAc (1/1) to afford tert-butyl 7-[4-chloro6-(4-methylpiperazin-1-yl)pyrimidin-2-yl]-2,7-diazaspiro[3.5]nonane-2-carboxylate (400 $\mathrm{mg}, 77 \%)$ as a brown solid. LCMS (ESI): [M+H]+: 437.4 .<smiles>[R20]C1CCN(c2nc(Cl)cc(N3CCN(C)CC3)n2)C1</smiles>

7-[4-Chloro-6-(4-methylpiperazin-1-yl)pyrimidin-2-yl]-2,7-diazaspiro[3.5]nonane (22-3): To a mixture of tert-butyl 7-[4-chloro-6-(4-methylpiperazin-1-yl)pyrimidin-2-yl]-2,7-diazaspiro[3.5]nonane-2-carboxylate (400 mg, $0.915 \mathrm{mmol}, 1$ equiv) in DCM $(10 \mathrm{~mL})$ was added TFA $(2 \mathrm{~mL})$. The resulting mixture was stirred at room temperature for 1 hour. The resulting mixture was concentrated under vacuum. This resulted in 7-[4-chloro-6-(4-methylpiperazin-1-yl)pyrimidin-2-yl]-2,7-diazaspiro[3.5]nonane (300 mg, 97\%) as a yellow crude oil. LCMS (ESI): [M+H] $]^{+}$337.3.<smiles>[R16]C1CCC2(CC1)CNC2c1nc(Cl)cc(N2CCN(C)CC2)n1</smiles>

22-3
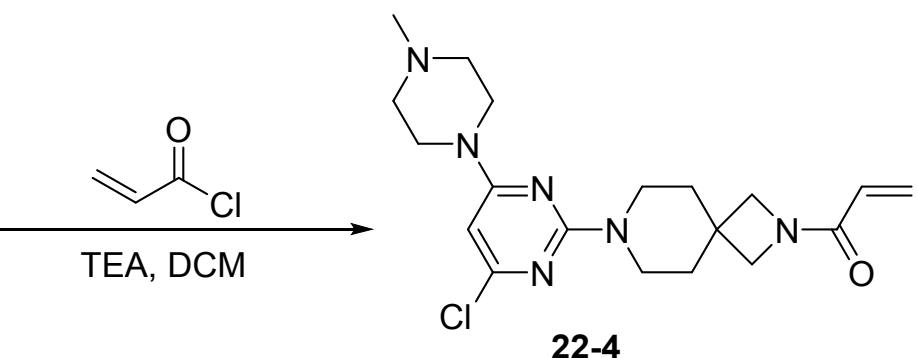

\section{1-[7-[4-Chloro-6-(4-methylpiperazin-1-yl)pyrimidin-2-yl]-2,7-diazaspiro[3.5]nonan-2-yl]prop-2-en-1-}

one (22-4): To a mixture of 7-[4-chloro-6-(4-methylpiperazin-1-yl)pyrimidin-2-yl]-2,7diazaspiro[3.5]nonane (300 mg, $0.891 \mathrm{mmol}, 1$ equiv) in DCM (6 mL) was added triethylamine ( $270 \mathrm{mg}$, $2.672 \mathrm{mmol}, 3$ equiv) and acryloyl chloride $\left(80 \mathrm{mg}, 0.891 \mathrm{mmol}, 1\right.$ equiv) dropwise at $0{ }^{\circ} \mathrm{C}$. The resulting mixture was stirred for 30 minutes at $0{ }^{\circ} \mathrm{C}$. The reaction was quenched with $\mathrm{MeOH}$ at $0{ }^{\circ} \mathrm{C}$. The resulting mixture was concentrated under vacuum. The residue was purified by silica gel column chromatography, eluting with $\mathrm{CH}_{2} \mathrm{Cl}_{2} / \mathrm{MeOH}$ (10/1) to afford 1-[7-[4-chloro-6-(4-methylpiperazin-1-yl)pyrimidin-2-yl]-2,7diazaspiro[3.5]nonan-2-yl]prop-2-en-1-one (150 mg, 43\%) as a yellow solid. LCMS (ESI): [M+H]+: 391.2. 


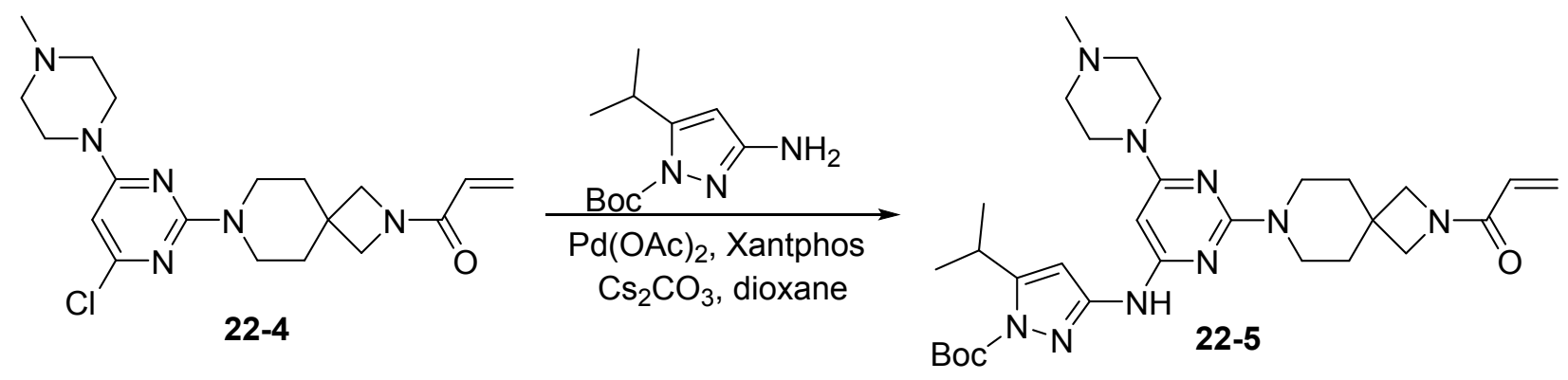

tert-Butyl 5-isopropyl-3-[[6-(4-methylpiperazin-1-yl)-2-[2-(prop-2-enoyl)-2,7-diazaspiro[3.5]nonan-7yl]pyrimidin-4-yl]amino]pyrazole-1-carboxylate (22-5): To a mixture of 1-[7-[4-chloro-6-(4methylpiperazin-1-yl)pyrimidin-2-yl]-2,7-diazaspiro[3.5]nonan-2-yl]prop-2-en-1-one $(110 \mathrm{mg}, \quad 0.281$ mmol, 1 equiv) and tert-butyl 3-amino-5-isopropylpyrazole-1-carboxylate (63 mg, $0.281 \mathrm{mmol}, 1$ equiv) in dioxane $\left(3 \mathrm{~mL}\right.$ ) were added $\mathrm{Cs}_{2} \mathrm{CO}_{3}(119 \mathrm{mg}, 0.366 \mathrm{mmol}, 1.3$ equiv), Xantphos ( $32 \mathrm{mg}, 0.056 \mathrm{mmol}, 0.2$ equiv) and $\mathrm{Pd}(\mathrm{OAc})_{2}(6.32 \mathrm{mg}, 0.028 \mathrm{mmol}, 0.1$ equiv) dropwise at room temperature. The resulting mixture was stirred at $80^{\circ} \mathrm{C}$ overnight. The resulting mixture was concentrated under vacuum. The residue was purified by silica gel column chromatography, eluting with $\mathrm{CH}_{2} \mathrm{Cl}_{2} / \mathrm{MeOH}(10 / 1)$ to afford tert-butyl 5isopropyl-3-[[6-(4-methylpiperazin-1-yl)-2-[2-(prop-2-enoyl)-2,7-diazaspiro[3.5]nonan-7-yl]pyrimidin-4yl]amino]pyrazole-1-carboxylate (100 $\mathrm{mg}, 61 \%)$ as a yellow solid. LCMS (ESI): [M+H]+: 580.1.
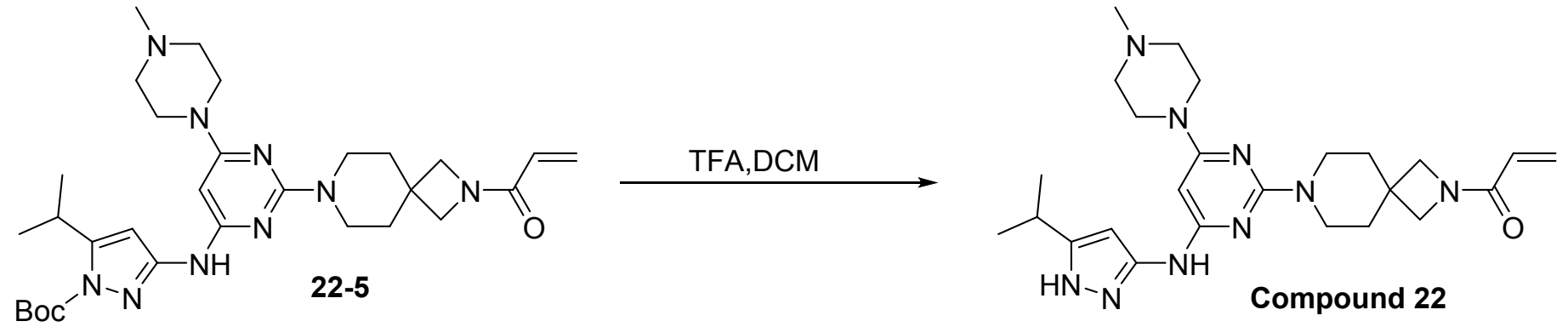

1-(7-[4-[(5-Isopropyl-1H-pyrazol-3-yl)amino]-6-(4-methylpiperazin-1-yl)pyrimidin-2-yl]-2,7diazaspiro[3.5]nonan-2-yl)prop-2-en-1-one (Compound 22): To a mixture of tert-butyl 5-isopropyl-3-[[6(4-methylpiperazin-1-yl)-2-[2-(prop-2-enoyl)-2,7-diazaspiro[3.5]nonan-7-yl]pyrimidin-4yl]amino]pyrazole-1-carboxylate (100 mg, $0.172 \mathrm{mmol}, 1$ equiv) in DCM (5 mL) was added TFA (1 mL). The resulting solution was stirred at room temperature for 1 hour. The resulting mixture was concentrated under vacuum. The crude product was purified by Prep-HPLC with the following conditions: Column, XBridge Shield RP18 OBD Column, 30*150mm, 5um; mobile phase, Water with $10 \mathrm{mmol} \mathrm{NH}_{4} \mathrm{HCO}_{3}$ and ACN (27.0\% MeCN up to $49.0 \%$ in $8 \mathrm{~min})$; UV $254 \mathrm{~nm}$. This resulted in $30.7 \mathrm{mg}$ (37\%) of 1-(7-[4-[(5isopropyl-1H-pyrazol-3-yl)amino]-6-(4-methylpiperazin-1-yl)pyrimidin-2-yl]-2,7-diazaspiro[3.5]nonan-2yl)prop-2-en-1-one as a white solid. LCMS (ESI): $[\mathrm{M}+\mathrm{H}]^{+}$: 480.4. ${ }^{1} \mathrm{H}$ NMR (300 MHz, CD $\left.{ }_{3} \mathrm{OD}\right) \delta 6.46-6.20$ $(\mathrm{m}, 2 \mathrm{H}), 6.13(\mathrm{~s}, 1 \mathrm{H}), 5.79-5.70(\mathrm{~m}, 1 \mathrm{H}), 4.95(\mathrm{~s}, 1 \mathrm{H}), 4.07(\mathrm{~s}, 2 \mathrm{H}), 3.83(\mathrm{~s}, 2 \mathrm{H}), 3.79-3.71(\mathrm{~m}, 4 \mathrm{H}), 3.56$ $(\mathrm{s}, 4 \mathrm{H}), 2.96(\mathrm{~s}, 1 \mathrm{H}), 2.50(\mathrm{~s}, 4 \mathrm{H}), 2.34(\mathrm{~s}, 3 \mathrm{H}), 1.81(\mathrm{~s}, 4 \mathrm{H}), 1.33-1.25(\mathrm{~m}, 6 \mathrm{H})$. 


\section{Compound 23:}
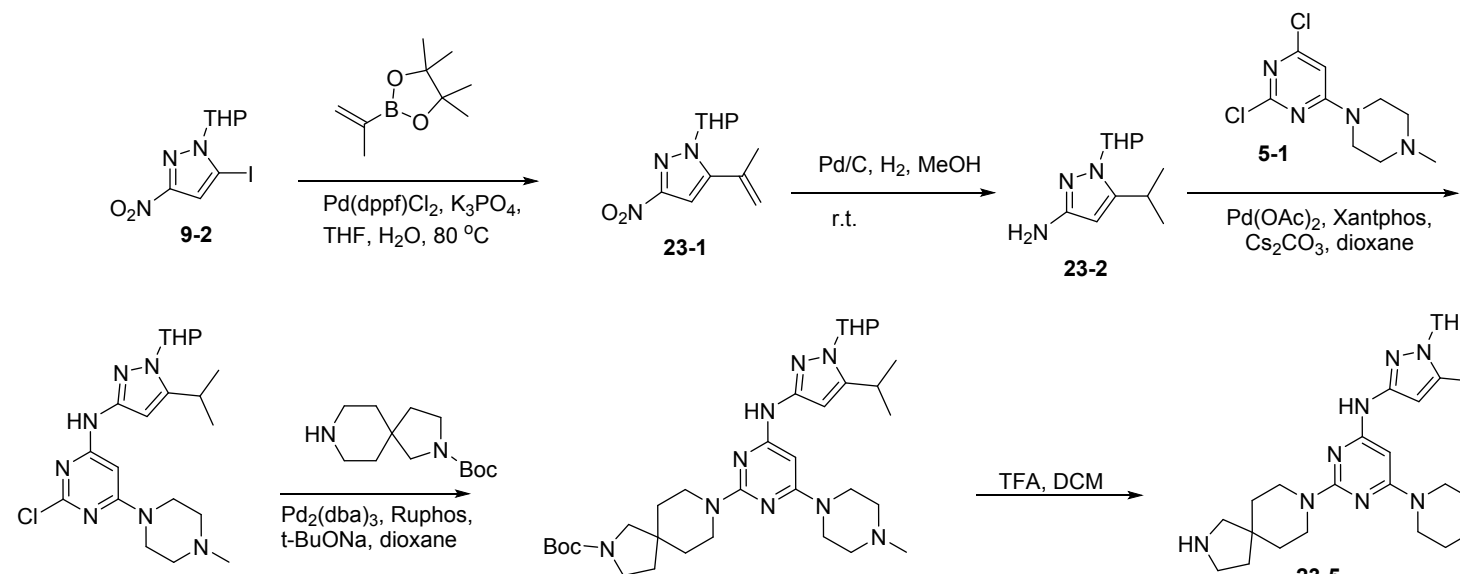

23-3
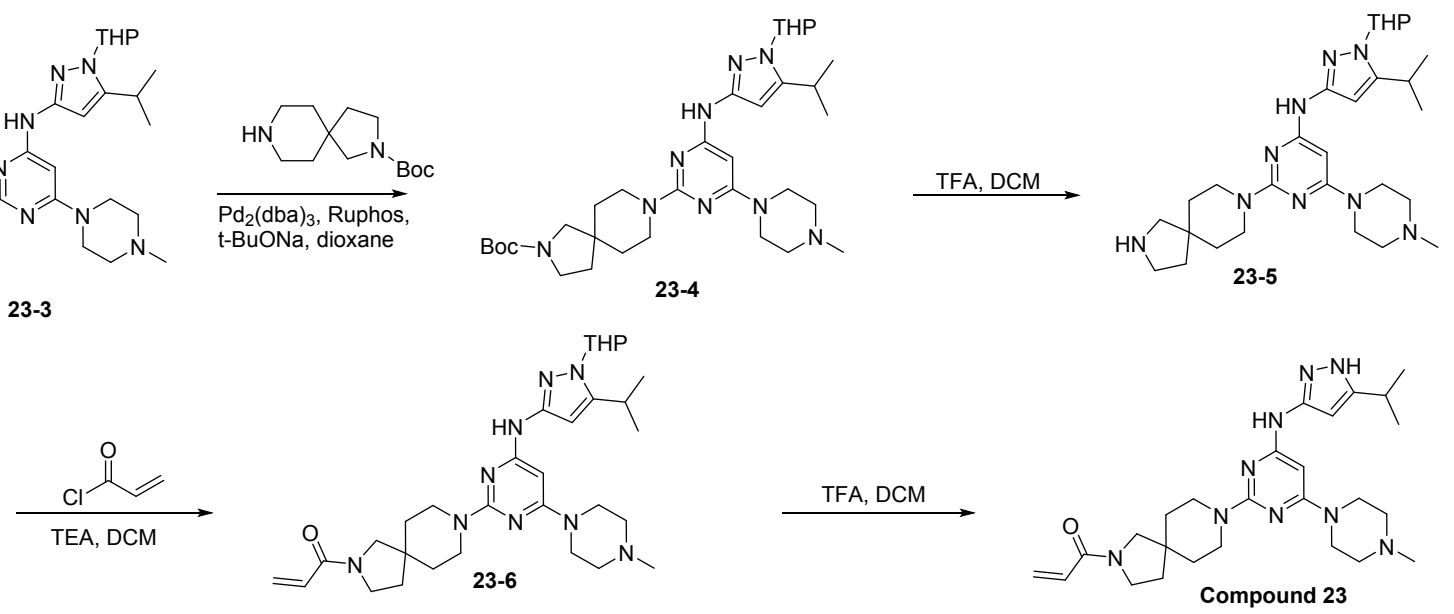<smiles>O=[N+]([O-])c1cc(I)n([TlH])n1</smiles>

9-2<smiles>C=C(C)B1OC(C)(C)C(C)(C)O1</smiles>

$\mathrm{Pd}(\mathrm{dppf}) \mathrm{Cl}_{2}, \mathrm{~K}_{3} \mathrm{PO}_{4}$,

THF, $\mathrm{H}_{2} \mathrm{O}, 80^{\circ} \mathrm{C}$<smiles>C=C(C)c1cc([N+](=O)[O-])nn1[TlH]</smiles>

23-1

3-Nitro-1-(oxan-2-yl)-5-(prop-1-en-2-yl)pyrazole (23-1): To a mixture of 5-iodo-3-nitro-1-(oxan-2yl)pyrazole (6.1 g, $18.88 \mathrm{mmol}, 1$ equiv) in THF (20 mL) were added 4,4,5,5-tetramethyl-2-(prop-1-en-2yl)-1,3,2-dioxaborolane (15.86 g, $94.401 \mathrm{mmol}, 5$ equiv), $\mathrm{Pd}(\mathrm{dppf}) \mathrm{Cl}_{2}$ (1.38 g, $0.002 \mathrm{mmol}, 0.1 \mathrm{equiv}$ ) and $\mathrm{K}_{3} \mathrm{PO}_{4}\left(12.02 \mathrm{~g}, 0.057 \mathrm{mmol}, 3\right.$ equiv) in $\mathrm{H}_{2} \mathrm{O}(2 \mathrm{~mL})$. The resulting mixture was stirred at $80{ }^{\circ} \mathrm{C}$ overnight under a nitrogen atmosphere. The resulting mixture was concentrated under reduced pressure. The residue was purified by silica gel column chromatography, eluting with PE/EtOAc (1/1) to afford 3-nitro1-(oxan-2-yl)-5-(prop-1-en-2-yl)pyrazole (3 g, 60\%) as a yellow oil. 
<smiles>C=C(C)c1cc([N+](=O)[O-])nn1[TlH]</smiles>

23-1

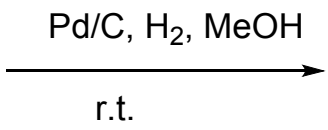

r.t.
$\mathrm{H}_{2} \mathrm{~N}$<smiles>CC(C)c1cc(N)nn1[TlH]</smiles>

23-2

5-Isopropyl-1-(oxan-2-yl)pyrazol-3-amine (23-2): To a mixture of 3-nitro-1-(oxan-2-yl)-5-(prop-1-en-2yl)pyrazole (7 g, $29.504 \mathrm{mmol}, 1$ equiv) in $\mathrm{MeOH}(20 \mathrm{~mL})$ was added $\mathrm{Pd} / \mathrm{C}$ (3.14 g). The resulting mixture was stirred at room temperature overnight under a hydrogen atmosphere. The solids were filtered out. The filtrate was concentrated under vacuum. The residue was purified by silica gel column chromatography, eluting with DCM / MeOH (10/1) to afford 5-isopropyl-1-(oxan-2-yl)pyrazol-3-amine (3.1 g, 50\%) as a yellow solid. LCMS (ESI): [M+H] $]^{+}: 210.1$.<smiles>CC(C)c1cc(N)nn1[Tl]</smiles>

23-2

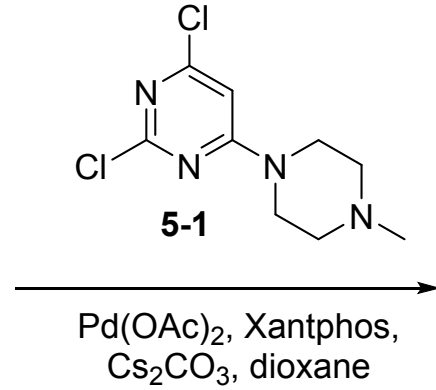

$\mathrm{Cs}_{2} \mathrm{CO}_{3}$, dioxane<smiles>CC(C)c1cc(Nc2cc(N3CCN(C)CC3)nc(Cl)n2)nn1[TlH]</smiles>

23-3

2-Chloro- $N$-[5-isopropyl-1-(oxan-2-yl)pyrazol-3-yl]-6-(4-methylpiperazin-1-yl)pyrimidin-4-amine (23-3): To a mixture of 5-isopropyl-1-(oxan-2-yl)pyrazol-3-amine ( $3 \mathrm{~g}, 14.334 \mathrm{mmol}, 1$ equiv) and 2,4-dichloro-6(4-methylpiperazin-1-yl)pyrimidine ( $3.54 \mathrm{~g}, 14.325 \mathrm{mmol}, 1$ equiv) in dioxane ( $30 \mathrm{~mL}$ ) were added $\mathrm{Cs}_{2} \mathrm{CO}_{3}$ (6.07 g, $18.634 \mathrm{mmol}, 1.3$ equiv), Xantphos ( $1.66 \mathrm{~g}, 2.867 \mathrm{mmol}, 0.2$ equiv) and $\mathrm{Pd}(\mathrm{OAc})_{2}(0.32 \mathrm{~g}, 1.433$ mmol, 0.1 equiv). The resulting mixture was stirred at $40^{\circ} \mathrm{C}$ for 2 hours under a nitrogen atmosphere. The residue was purified by silica gel column chromatography, eluting with $\mathrm{CH}_{2} \mathrm{Cl}_{2} / \mathrm{MeOH}(10 / 1)$ to afford 2.1 $\mathrm{g}$ of crude product. The crude product was purified by Prep-ACHIRAL-SFC with the following conditions: Column: DAICEL DCpak P4VP(02), 30*250mm,5um; Mobile Phase A: $\mathrm{CO}_{2}$, Mobile Phase B: $\mathrm{MeOH}(2 \mathrm{mM}$ $\mathrm{NH}_{3}-\mathrm{MeOH}$ ); Flow rate:50 mL/min; Gradient:25\% B; $254 \mathrm{~nm}$; This resulted in 2-chloro- $\mathrm{N}$-[5-isopropyl-1(oxan-2-yl)pyrazol-3-yl]-6-(4-methylpiperazin-1-yl)pyrimidin-4-amine (1.3 g, 22\%) as a white solid. ${ }^{1} \mathrm{H}$ NMR (300 MHz, CD $\left.{ }_{3} \mathrm{OD}\right) \delta 6.81(\mathrm{~s}, 1 \mathrm{H}), 6.01(\mathrm{~s}, 1 \mathrm{H}), 5.44-5.34(\mathrm{~m}, 1 \mathrm{H}), 4.06-3.96(\mathrm{~m}, 1 \mathrm{H}), 3.84-3.46(\mathrm{~m}, 5 \mathrm{H})$, $3.20-3.02(\mathrm{~m}, 1 \mathrm{H}), 2.57-2.46(\mathrm{~m}, 4 \mathrm{H}), 2.46-2.34(\mathrm{~m}, 1 \mathrm{H}), 2.35(\mathrm{~s}, 3 \mathrm{H}), 2.17-2.07(\mathrm{~m}, 1 \mathrm{H}), 2-1.88(\mathrm{~m}$, $1 \mathrm{H}), 1.89-1.70(\mathrm{~m}, 1 \mathrm{H}), 1.69-1.56(\mathrm{~m}, 2 \mathrm{H}), 1.30(\mathrm{~d}, J=6.9 \mathrm{~Hz}, 6 \mathrm{H})$. Structures were assigned by $1 \mathrm{H}$ NMR analysis after de-chlorination. 
<smiles>[Z19]c1nc(Nc2cc(C(C)C)n([TlH])n2)cc(N2CCN(C)CC2)n1</smiles>

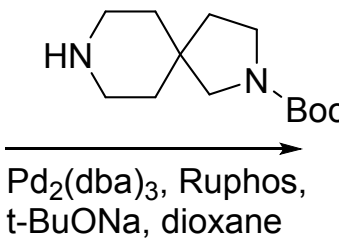

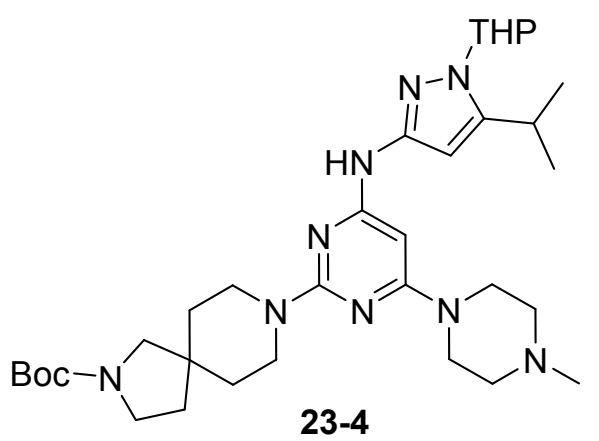

23-4

tert-Butyl 8-(4-[[5-isopropyl-1-(oxan-2-yl)pyrazol-3-yl]amino]-6-(4-methylpiperazin-1-yl)pyrimidin-2yl)-2,8-diazaspiro[4.5]decane-2-carboxylate (23-4): To a mixture of 2-chloro- $N$-[5-isopropyl-1-(oxan-2yl)pyrazol-3-yl]-6-(4-methylpiperazin-1-yl)pyrimidin-4-amine (120 mg, $0.29 \mathrm{mmol}, 1$ equiv) and tert-butyl 2,8-diazaspiro[4.5]decane-2-carboxylate (103 $\mathrm{mg}, 0.43 \mathrm{mmol}, 1.5$ equiv) in dioxane (4 mL) were added $t$ BuONa (41 mg, $0.43 \mathrm{mmol}, 1.5$ equiv), Ruphos ( $27 \mathrm{mg}, 0.06 \mathrm{mmol}, 0.2$ equiv) and $\mathrm{Pd}_{2}(\mathrm{dba})_{3}(26 \mathrm{mg}, 0.03$ mmol, 0.1 equiv). The resulting mixture was stirred at $100{ }^{\circ} \mathrm{C}$ for 1 hour under $\mathrm{N}_{2}$. The resulting mixture was concentrated under vacuum. The residue was applied onto a silica gel column eluting with $\mathrm{CH}_{2} \mathrm{Cl}_{2} / \mathrm{MeOH}$ (12/1). This resulted in $120 \mathrm{mg}$ (66\%) of tert-butyl 8-(4-[[5-isopropyl-1-(oxan-2-yl)pyrazol3-yl]amino]-6-(4-methylpiperazin-1-yl)pyrimidin-2-yl)-2,8-diazaspiro[4.5]decane-2-carboxylate as a yellow solid. LCMS (ESI): [M+H]+: 624.4.<smiles>CC(C)c1cc(Nc2cc(N3CCN(C)CC3)nc(N3CCC4(CCN(C(=O)OC(C)(C)C)C4)CC3)n2)nn1[TlH]</smiles>
$\underset{\mathrm{TFA}, \mathrm{DCM}}{\longrightarrow}$<smiles>CC(C)c1cc(Nc2cc(N3CCN(C)CC3)nc(N3CCC4(CCNC4)CC3)n2)nn1[Tl]</smiles>

\section{2-[2,8-Diazaspiro[4.5]decan-8-yl]-N-[5-isopropyl-1-(oxan-2-yl)pyrazol-3-yl]-6-(4-methylpiperazin-1-}

yl)pyrimidin-4-amine (23-5): To a mixture of tert-butyl 8-(4-[[5-isopropyl-1-(oxan-2-yl)pyrazol-3yl]amino]-6-(4-methylpiperazin-1-yl)pyrimidin-2-yl)-2,8-diazaspiro[4.5]decane-2-carboxylate $(120 \mathrm{mg}$, $0.19 \mathrm{mmol}, 1$ equiv) in DCM $(3 \mathrm{~mL})$ were added TFA $(1 \mathrm{~mL})$ at $0{ }^{\circ} \mathrm{C}$. The resulting mixture was stirred at 0 ${ }^{\circ} \mathrm{C}$ for 1 hour. The resulting mixture was concentrated under vacuum. This resulted in $100 \mathrm{mg}(81 \%)$ of 2[2,8-diazaspiro[4-5]decan-8-yl]-N-[5-isopropyl-1-(oxan-2-yl)pyrazol-3-yl]-6-(4-methylpiperazin-1yl)pyrimidin-4-amine as a yellow crude oil. LCMS (ESI): [M+H]+: 524.4. 
<smiles>CC(C)c1cc(Nc2cc(N3CCN(C)CC3)nc(N3CCC4(CCNC4)CC3)n2)nn1[Tl]</smiles>

23-5

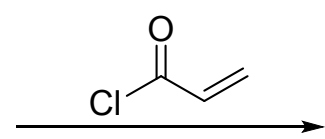

TEA, DCM

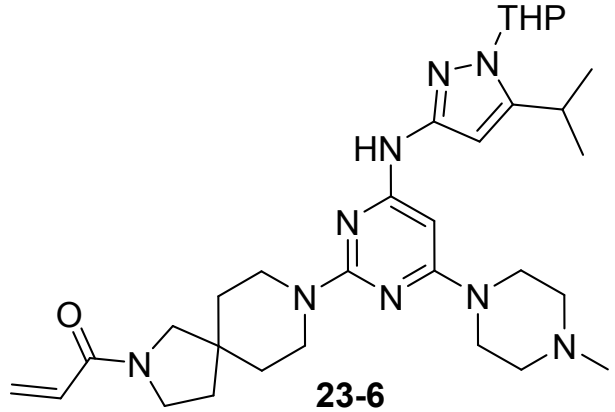

23-6

1-[8-(4-[[5-Isopropyl-1-(oxan-2-yl)pyrazol-3-yl]amino]-6-(4-methylpiperazin-1-yl)pyrimidin-2-yl)-2,8diazaspiro[4.5]decan-2-yl]prop-2-en-1-one (23-6): To a mixture of 2-[2,8-diazaspiro[4.5]decan-8-yl]- $N$-[5isopropyl-1-(oxan-2-yl)pyrazol-3-yl]-6-(4-methylpiperazin-1-yl)pyrimidin-4-amine (100 mg, $0.19 \mathrm{mmol}, 1$ equiv) and triethylamine (58 mg, $0.57 \mathrm{mmol}, 3$ equiv) in DCM (4 mL) were added acryloyl chloride (19 $\mathrm{mg}$, $0.21 \mathrm{mmol}, 1.1$ equiv) at $0{ }^{\circ} \mathrm{C}$. The resulting mixture was stirred at $0{ }^{\circ} \mathrm{C}$ for 1 hour. The reaction was quenched with $\mathrm{MeOH}$. The resulting mixture was concentrated under vacuum. The residue was applied onto a silica gel column eluting with $\mathrm{CH}_{2} \mathrm{Cl}_{2} / \mathrm{MeOH}$ (5/1). This resulted in $60 \mathrm{mg}$ (49\%) of 1-[8-(4-[[5isopropyl-1-(oxan-2-yl)pyrazol-3-yl]amino]-6-(4-methylpiperazin-1-yl)pyrimidin-2-yl)-2,8diazaspiro[4.5]decan-2-yl]prop-2-en-1-one as a yellow solid. LCMS (ESI): [M+H]+: 578.3.

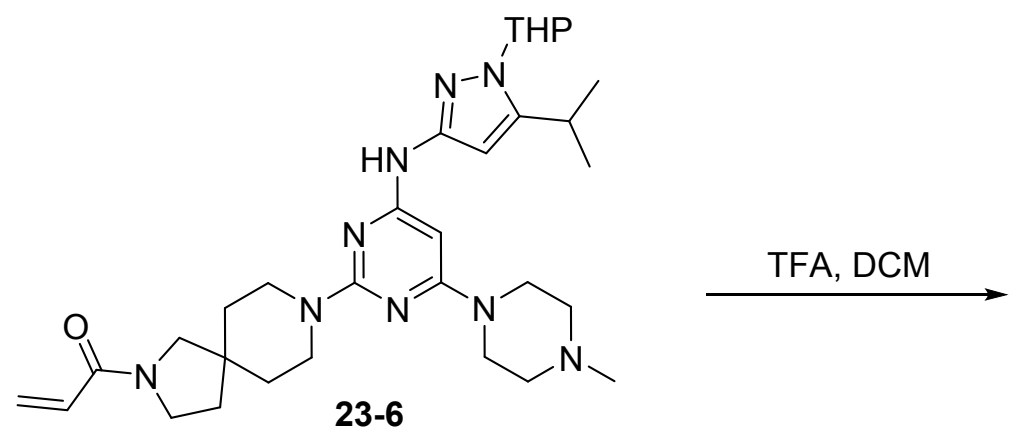

23-6<smiles>C=CC(=O)N1CCC2(CCN(c3nc(Nc4cc(C(C)C)[nH]n4)cc(N4CCN(C)CC4)n3)CC2)C1</smiles>

Compound 23

1-(8-[4-[(5-Isopropyl-1H-pyrazol-3-yl)amino]-6-(4-methylpiperazin-1-yl)pyrimidin-2-yl]-2,8-

diazaspiro[4.5]decan-2-yl)prop-2-en-1-one (Compound 23): To a mixture of 1-[8-(4-[[5-isopropyl-1(oxan-2-yl)pyrazol-3-yl]amino]-6-(4-methylpiperazin-1-yl)pyrimidin-2-yl)-2,8-diazaspiro[4.5]decan-2-

yl]prop-2-en-1-one $\left(60 \mathrm{mg}, 0.1 \mathrm{mmol}, 1\right.$ equiv) in DCM $(3 \mathrm{~mL})$ were added TFA $(1 \mathrm{~mL})$ at $0{ }^{\circ} \mathrm{C}$. The resulting mixture was stirred at $40^{\circ} \mathrm{C}$ for 1 hour. The resulting mixture was concentrated under vacuum. The crude product was purified by Prep-HPLC with the following conditions: Column: XBridge Prep OBD C18 Column, 30×150mm 5um; Mobile Phase A:Water(10MMOL/L $\left.\mathrm{NH}_{4} \mathrm{HCO}_{3}\right)$, Mobile Phase B:ACN; Flow rate:60 $\mathrm{mL} / \mathrm{min}$; Gradient:44 B to 74 B in $7 \mathrm{~min} ; 254 \mathrm{~nm}$. This resulted in $19.7 \mathrm{mg}$ (37\%) of 1-(8-[4-[(5-isopropyl1H-pyrazol-3-yl)amino]-6-(4-methylpiperazin-1-yl)pyrimidin-2-yl]-2,8-diazaspiro[4.5]decan-2-yl)prop-2en-1-one as a yellow solid. LCMS (ESI): $[\mathrm{M}+\mathrm{H}]^{+}:$494.3. ${ }^{1} \mathrm{H}$ NMR (300 MHz, $\left.\mathrm{CD}_{3} \mathrm{OD}\right) \delta$ 6.71-6.54 (m, $\left.1 \mathrm{H}\right)$, 6.34-6.21 (m, 1H), $6.13(\mathrm{~s}, 1 \mathrm{H}), 5.80-5.69(\mathrm{~m}, 1 \mathrm{H}), 4.90-4.50(\mathrm{~m}, 1 \mathrm{H}), 3.89-3.50(\mathrm{~m}, 11 \mathrm{H}), 3.44(\mathrm{~s}, 1 \mathrm{H}), 2.95$ $(\mathrm{s}, 1 \mathrm{H}), 2.54-2.45(\mathrm{~m}, 4 \mathrm{H}), 2.33(\mathrm{~s}, 3 \mathrm{H}), 2.01-1.83(\mathrm{~m}, 2 \mathrm{H}), 1.61(\mathrm{~s}, 4 \mathrm{H}), 1.28(\mathrm{~d}, J=6.9 \mathrm{~Hz}, 6 \mathrm{H})$. 


\section{Compound 24:}
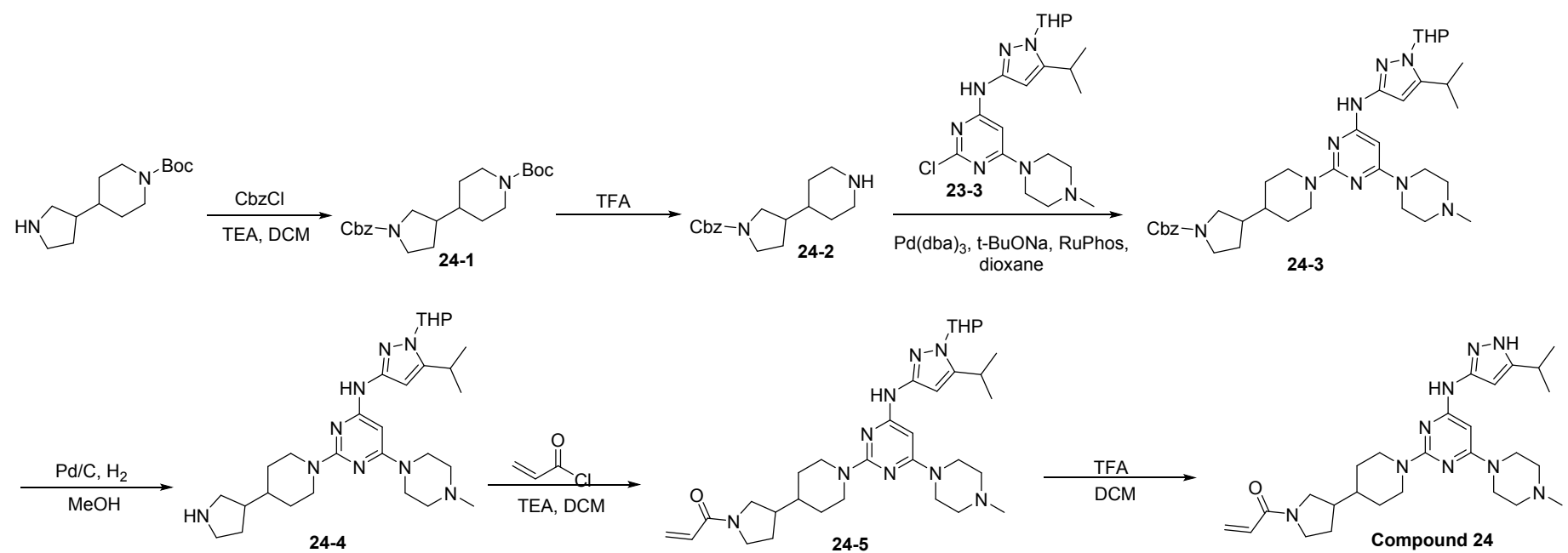<smiles>[Z10]CC1CCN(C(=O)COC(=O)OC(C)(C)C)C1C1CCN(C(=O)OC(C)(C)C)CC1</smiles>

tert-Butyl 4-[1-[(benzyloxy)carbonyl]pyrrolidin-3-yl]piperidine-1-carboxylate (24-1): To a mixture of tert-butyl 4-(pyrrolidin-3-yl)piperidine-1-carboxylate $(900 \mathrm{mg}, 3.538 \mathrm{mmol}, 1$ equiv) in DCM (10 mL) were added triethylamine (1074 mg, $10.614 \mathrm{mmol}, 3$ equiv) and benzyl chloroformate ( $905 \mathrm{mg}, 5.307 \mathrm{mmol}$, 1.5 equiv) dropwise at $0{ }^{\circ} \mathrm{C}$. The resulting mixture was stirred at $0{ }^{\circ} \mathrm{C}$ for 1 hour. The resulting mixture was concentrated under vacuum. The residue was purified by silica gel column chromatography, eluted with $\mathrm{ACN} / \mathrm{H}_{2} \mathrm{O}(2 / 1)$ to afford tert-butyl 4-[1-[(benzyloxy)carbonyl]pyrrolidin-3-yl]piperidine-1-carboxylate (700 mg, 51\%) as a white solid. LCMS (ESI): [M+H]+: 389.15.<smiles>[Z10]CC1CCN(C(=O)OC(C)(C)C)CC1</smiles>

Benzyl 3-(piperidin-4-yl)pyrrolidine-1-carboxylate (24-2): To a mixture of tert-butyl 4-[1[(benzyloxy)carbonyl]pyrrolidin-3-yl]piperidine-1-carboxylate (700 mg, $1.802 \mathrm{mmol}, 1$ equiv) in DCM (10 $\mathrm{mL}$ ) was added TFA ( $2 \mathrm{~mL}$ ). The resulting mixture was stirred at room temperature for 1 hour. The resulting mixture was concentrated under vacuum. This resulted in benzyl 3-(piperidin-4-yl)pyrrolidine-1carboxylate (510 $\mathrm{mg}, 98 \%$ ) as a yellow crude oil. LCMS (ESI): [M+H]+: 289.1. 
<smiles>[R4][X]C1CCN(C(=O)OCc2ccccc2)C1</smiles>

24-2

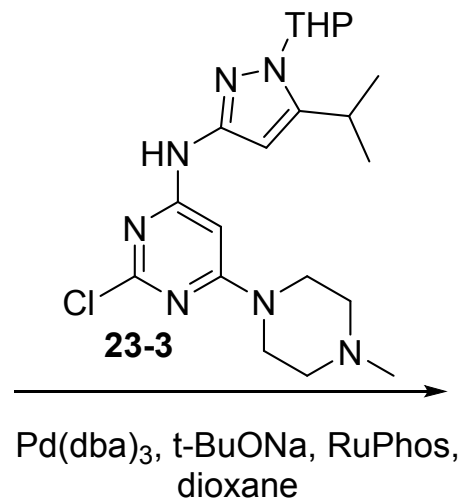

dioxane<smiles>[Z13]C(C)c1cc(Nc2cc(N3CCN(C)CC3)nc(N3CCC(C4CCN5CC=CC=CC=C4COC5=O)CC3)n2)nn1[TlH]</smiles>

24-3

Benzyl 3-[1-(4-[[5-isopropyl-1-(oxan-2-yl)pyrazol-3-yl]amino]-6-(4-methylpiperazin-1-yl)pyrimidin-2yl)piperidin-4-yl]pyrrolidine-1-carboxylate (24-3): To a mixture of benzyl 3-(piperidin-4-yl)pyrrolidine-1carboxylate (140 mg, $0.485 \mathrm{mmol}, 1$ equiv) and 2-chloro- $N$-[5-isopropyl-1-(oxan-2-yl)pyrazol-3-yl]-6-(4methylpiperazin-1-yl)pyrimidin-4-amine (203 mg, $0.485 \mathrm{mmol}, 1$ equiv) in dioxane $(3 \mathrm{~mL}$ ) were added $t$ BuONa (69 mg, 0.728 mmol, 1.5 equiv) and $\mathrm{Pd}_{2}(\mathrm{dba})_{3}(44 \mathrm{mg}, 0.049 \mathrm{mmol}, 0.1$ equiv). The resulting mixture was stirred at $100{ }^{\circ} \mathrm{C}$ overnight under a nitrogen atmosphere. The residue was purified by Prep$\mathrm{TLC}\left(\mathrm{CH}_{2} \mathrm{Cl}_{2} / \mathrm{MeOH}=10 / 1\right)$ to afford benzyl 3-[1-(4-[[5-isopropyl-1-(oxan-2-yl)pyrazol-3-yl]amino]-6-(4methylpiperazin-1-yl)pyrimidin-2-yl)piperidin-4-yl]pyrrolidine-1-carboxylate (100 $\mathrm{mg}, 21 \%$ ) as a yellow solid. LCMS (ESI): [M+H]+: 672.4 .

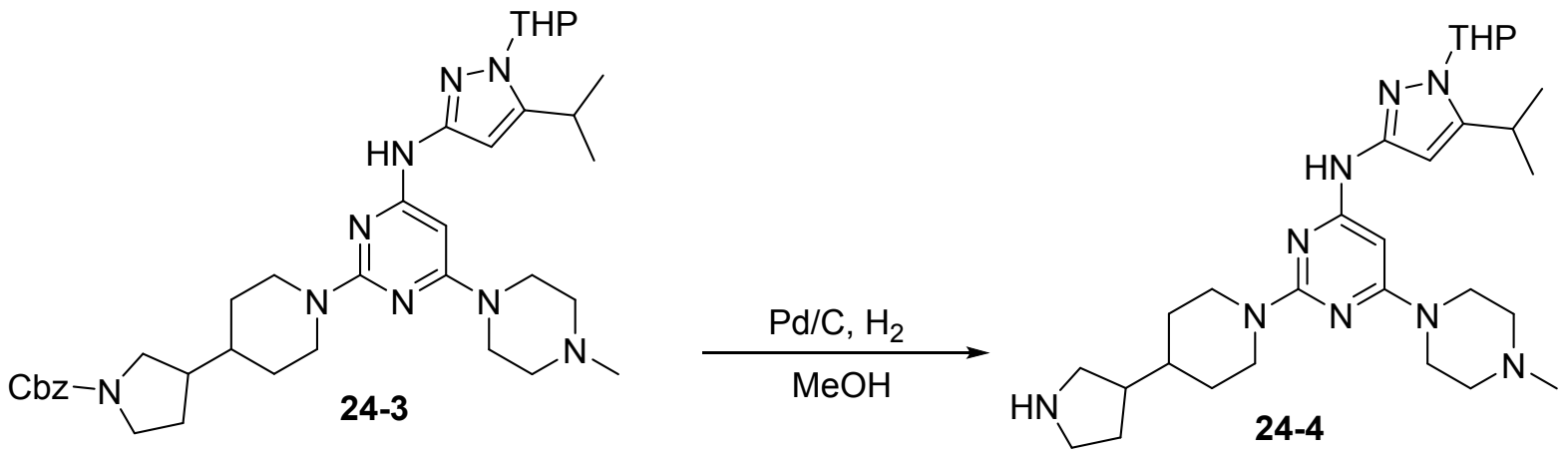

\section{N-[5-Isopropyl-1-(oxan-2-yl)pyrazol-3-yl]-6-(4-methylpiperazin-1-yl)-2-[4-(pyrrolidin-3-yl)piperidin-1-} yl]pyrimidin-4-amine (24-4): To a mixture of benzyl 3-[1-(4-[[5-isopropyl-1-(oxan-2-yl)pyrazol-3yl]amino]-6-(4-methylpiperazin-1-yl)pyrimidin-2-yl)piperidin-4-yl]pyrrolidine-1-carboxylate $(80 \mathrm{mg}, 0.119$ mmol, 1 equiv) in $\mathrm{MeOH}(5 \mathrm{~mL})$ was added $\mathrm{Pd} / \mathrm{C}(80 \mathrm{mg})$. The resulting mixture was stirred at room temperature for 2 hours under a hydrogen atmosphere. The resulting mixture was filtered, the filter cake was washed with $\mathrm{MeOH}$. The filtrate was concentrated under reduced pressure. The residue was purified by Prep-TLC $\left(\mathrm{CH}_{2} \mathrm{Cl}_{2} / \mathrm{MeOH}=5 / 1\right)$ to afford $N$-[5-isopropyl-1-(oxan-2-yl)pyrazol-3-yl]-6-(4methylpiperazin-1-yl)-2-[4-(pyrrolidin-3-yl)piperidin-1-yl]pyrimidin-4-amine (60 mg, 94\%) as a yellow solid. LCMS (ESI): $[\mathrm{M}+\mathrm{H}]^{+}: 538.4$. 

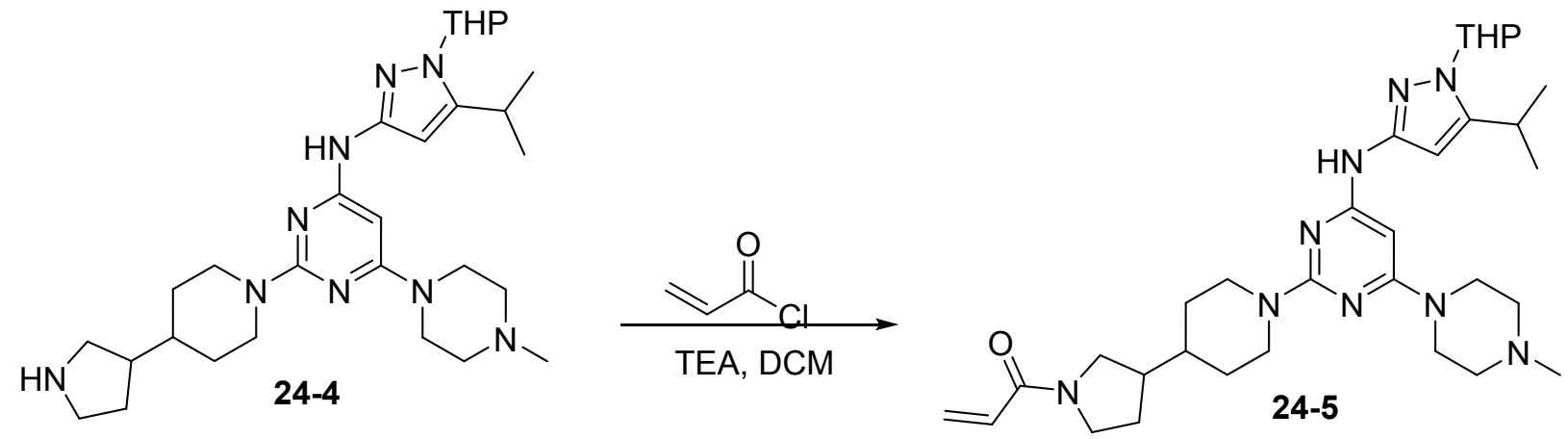

1-[3-[1-(4-[[5-Isopropyl-1-(oxan-2-yl)pyrazol-3-yl]amino]-6-(4-methylpiperazin-1-yl)pyrimidin-2yl)piperidin-4-yl]pyrrolidin-1-yl]prop-2-en-1-one (24-5): To a mixture of $\mathrm{N}$-[5-isopropyl-1-(oxan-2yl)pyrazol-3-yl]-6-(4-methylpiperazin-1-yl)-2-[4-(pyrrolidin-3-yl)piperidin-1-yl]pyrimidin-4-amine (50 mg, $0.093 \mathrm{mmol}, 1$ equiv) in DCM ( $3 \mathrm{~mL}$ ) were added triethylamine ( $28 \mathrm{mg}, 0.279 \mathrm{mmol}, 3$ equiv) and acryloyl chloride ( $9 \mathrm{mg}, 0.093 \mathrm{mmol}, 1$ equiv) dropwise at $0{ }^{\circ} \mathrm{C}$. The resulting mixture was stirred at $0{ }^{\circ} \mathrm{C}$ for 1 hour. The reaction was quenched with $\mathrm{MeOH}$ at $0{ }^{\circ} \mathrm{C}$. The resulting mixture was concentrated under vacuum. The residue was purified by Prep-TLC $\left(\mathrm{CH}_{2} \mathrm{Cl}_{2} / \mathrm{MeOH}=10 / 1\right)$ to afford 1-[3-[1-(4-[[5-isopropyl-1-(oxan-2yl)pyrazol-3-yl]amino]-6-(4-methylpiperazin-1-yl)pyrimidin-2-yl)piperidin-4-yl]pyrrolidin-1-yl]prop-2-en1-one (35 mg, 64\%) as a yellow solid. LCMS (ESI): [M+H] $]^{+}: 592.4$.

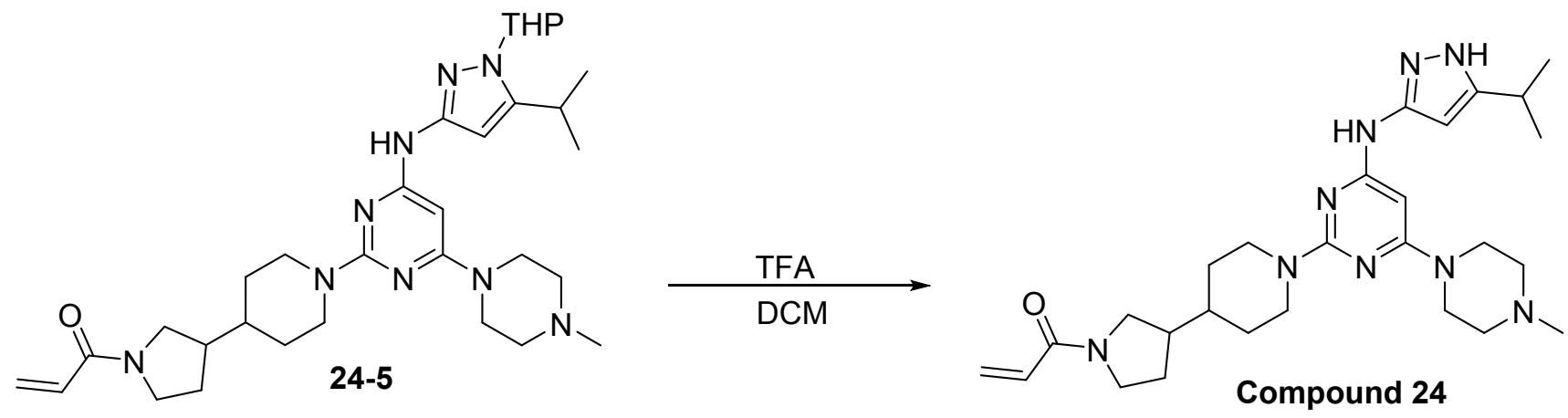

1-[3-(1-[4-[(5-Isopropyl-1H-pyrazol-3-yl)amino]-6-(4-methylpiperazin-1-yl)pyrimidin-2-yl]piperidin-4yl)pyrrolidin-1-yl]prop-2-en-1-one (Compound 24): To a mixture of 1-[3-[1-(4-[[5-isopropyl-1-(oxan-2yl)pyrazol-3-yl]amino]-6-(4-methylpiperazin-1-yl)pyrimidin-2-yl)piperidin-4-yl]pyrrolidin-1-yl]prop-2-en1-one ( $35 \mathrm{mg}, 0.059 \mathrm{mmol}, 1$ equiv) in DCM $(2 \mathrm{~mL})$ was added TFA $(0.5 \mathrm{~mL})$ dropwise at room temperature. The resulting mixture was stirred at $40{ }^{\circ} \mathrm{C}$ for 1 hour. The resulting mixture was concentrated under vacuum. The residue was purified by Prep-HPLC with the following conditions: Column: Xselect CSH OBD Column 30*150mm 5um, Mobile Phase A: Water (10MMOL/L NH$H_{4} \mathrm{HCO}_{3}$ ), Mobile Phase B: ACN:MEOH=4:1; Flow rate: $60 \mathrm{~mL} / \mathrm{min}$; Gradient: $37 \mathrm{~B}$ to $57 \mathrm{~B}$ in $8 \mathrm{~min} ; 220 \mathrm{~nm}$. This resulted in 1-[3-(1-[4-[(5-isopropyl1H-pyrazol-3-yl)amino]-6-(4-methylpiperazin-1-yl)pyrimidin-2-yl]piperidin-4-yl)pyrrolidin-1-yl]prop-2-en1-one (6.2 mg, 21\%) as a white solid. LCMS (ESI): [M+H] ${ }^{+}: 508.4 .{ }^{1} \mathrm{H}$ NMR (300 MHz, CD3OD) $\delta 6.69-6.51$ 
$(\mathrm{m}, 1 \mathrm{H}), 6.30-6.07(\mathrm{~m}, 2 \mathrm{H}), 5.77-5.67(\mathrm{~m}, 1 \mathrm{H}), 4.69(\mathrm{~s}, 2 \mathrm{H}), 3.92-3.60(\mathrm{~m}, 2 \mathrm{H}), 3.53(\mathrm{~d}, \mathrm{~J}=4.8 \mathrm{~Hz}, 5 \mathrm{H})$, $3.22(\mathrm{~d}, \mathrm{~J}=9.9 \mathrm{~Hz}, 1 \mathrm{H}), 2.80(\mathrm{~s}, 3 \mathrm{H}), 2.50-2.47(\mathrm{~m}, 4 \mathrm{H}), 2.32(\mathrm{~s}, 3 \mathrm{H}), 2.15(\mathrm{~d}, J=5.7 \mathrm{~Hz}, 2 \mathrm{H}), 1.83-1.35$ $(\mathrm{m}, 4 \mathrm{H}), 1.27(\mathrm{~d}, J=6.9 \mathrm{~Hz}, 9 \mathrm{H})$.

\section{Compound 6a:}

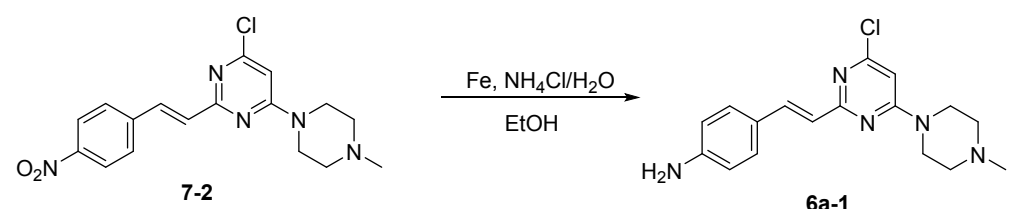

6a-1

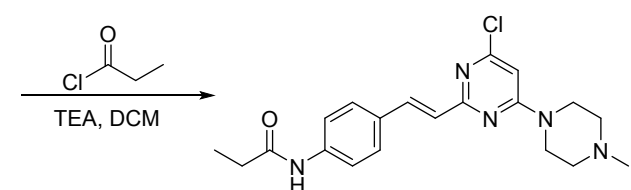

6a-2

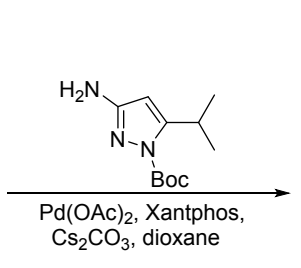<smiles></smiles>

6a-3

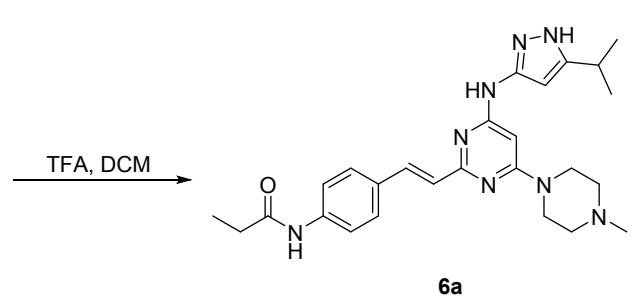<smiles>CN1CCN(c2cc(Cl)nc(/C=C/c3ccc([N+](=O)[O-])cc3)n2)CC1</smiles>

$7 a$

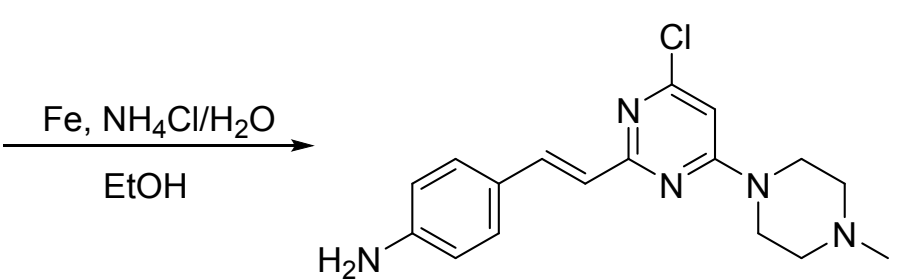

$6 a-1$

\section{4-[(E)-2-[4-chloro-6-(4-methylpiperazin-1-yl)pyrimidin-2-yl]ethenyl]aniline: To a mixture of 4-chloro-6-} (4-methylpiperazin-1-yl)-2-[(E)-2-(4-nitrophenyl)ethenyl]pyrimidine (200 mg, $0.56 \mathrm{mmol}, 1$ equiv) and iron (310 mg, $5.56 \mathrm{mmol}, 10$ equiv) in $\mathrm{EtOH}(5 \mathrm{~mL})$ was added saturated aqueous $\mathrm{NH}_{4} \mathrm{Cl}(1 \mathrm{~mL})$. The resulting mixture was stirred at $80^{\circ} \mathrm{C}$ for 3 hours. The resulting mixture was concentrated under vacuum. The residue was applied onto a silica gel column with $\mathrm{CH}_{2} \mathrm{Cl}_{2} / \mathrm{MeOH}$ (10/1). This resulted in 110 $\mathrm{mg}(55 \%)$ of 4-[(E)-2-[4-chloro-6-(4-methylpiperazin-1-yl)pyrimidin-2-yl]ethenyl]aniline as a yellow solid. LCMS (ESI): $[\mathrm{M}+\mathrm{H}]^{+}: 330.1$.<smiles>CN1CCN(c2cc(Cl)nc(/C=C/c3ccc(N)cc3)n2)CC1</smiles>

6a-1<smiles>CCC(=O)Nc1ccc(/C=C/c2nc(Cl)cc(N3CCN(C)CC3)n2)cc1</smiles>

6a-2 
$N$-[4-[(E)-2-[4-chloro-6-(4-methylpiperazin-1-yl)pyrimidin-2-yl]ethenyl]phenyl]propanamide: To a mixture of 4-[(E)-2-[4-chloro-6-(4-methylpiperazin-1-yl)pyrimidin-2-yl]ethenyl]aniline (110 mg, 0.33 mmol, 1 equiv) in DCM ( $3 \mathrm{~mL})$ were added triethylamine $(0.5 \mathrm{~mL})$ and propanoyl chloride $(62 \mathrm{mg}, 0.68$ mmol, 2 equiv) at $0{ }^{\circ} \mathrm{C}$. The resulting mixture was stirred at $0^{\circ} \mathrm{C}$ for 1 hour. The resulting mixture was quenched with $\mathrm{MeOH}$. The resulting mixture was concentrated under vacuum. The residue was applied onto a silica gel column eluting with $\mathrm{CH}_{2} \mathrm{Cl}_{2} / \mathrm{MeOH}$ (10/1). This resulted in $130 \mathrm{mg}$ (92\%) of N-[4-[(E)-2[4-chloro-6-(4-methylpiperazin-1-yl)pyrimidin-2-yl]ethenyl]phenyl]propanamide as a yellow solid. LCMS (ESI): $[\mathrm{M}+\mathrm{H}]^{+}: 386.1$.

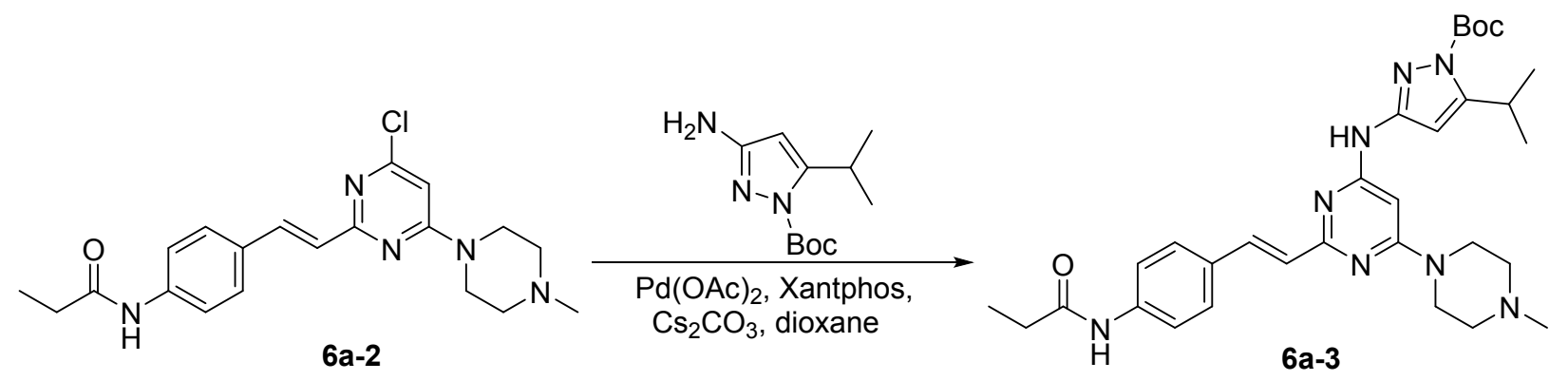

tert-Butyl 5-isopropyl-3-[[6-(4-methylpiperazin-1-yl)-2-[(E)-2-(4-

propanamidophenyl)ethenyl]pyrimidin-4-yl]amino]pyrazole-1-carboxylate: To a mixture of $N$-[4-[(E)-2-

[4-chloro-6-(4-methylpiperazin-1-yl)pyrimidin-2-yl]ethenyl]phenyl]propanamide (65 mg, 0.17 mmol, 1 equiv) and tert-butyl 3-amino-5-isopropylpyrazole-1-carboxylate (46 mg, $0.2 \mathrm{mmol}, 1.2$ equiv) in dioxane (3 mL) were added $\mathrm{Cs}_{2} \mathrm{CO}_{3}(165 \mathrm{mg}, 0.51 \mathrm{mmol}, 3$ equiv), Xantphos (19 mg, $0.03 \mathrm{mmol}, 0.2$ equiv) and $\mathrm{Pd}(\mathrm{OAc})_{2}\left(4 \mathrm{mg}, 0.02 \mathrm{mmol}, 0.1\right.$ equiv). The resulting mixture was stirred at $80^{\circ} \mathrm{C}$ overnight under $\mathrm{N}_{2}$. The resulting mixture was concentrated under vacuum. The residue was applied onto a silica gel column eluting with $\mathrm{CH}_{2} \mathrm{Cl}_{2} / \mathrm{MeOH}$ (12/1). This resulted in $30 \mathrm{mg}$ (29\%) of tert-butyl 5-isopropyl-3-[[6-(4methylpiperazin-1-yl)-2-[(E)-2-(4-propanamidophenyl)ethenyl]pyrimidin-4-yl]amino]pyrazole-1carboxylate as a yellow solid. LCMS (ESI): [M+H]+: 575.4 .<smiles>CCC(=O)Nc1ccc(/C=C/c2nc(Nc3ccc(/C=C/c4cc(C(C)C)n(C(=O)OC(C)(C)C)n4)cc3)cc(N3CCN(C)CC3)n2)cc1</smiles> 


\section{$N$-[4-[(E)-2-[4-[(5-isopropyl-1H-pyrazol-3-yl)amino]-6-(4-methylpiperazin-1-yl)pyrimidin-2-}

yl]ethenyl]phenyl]propanamide: To a mixture of tert-butyl 5-isopropyl-3-[[6-(4-methylpiperazin-1-yl)-2[(E)-2-(4-propanamidophenyl)ethenyl]pyrimidin-4-yl]amino]pyrazole-1-carboxylate (30 mg, $0.05 \mathrm{mmol}$, 1 equiv) in DCM $(1 \mathrm{~mL})$ were added TFA $(0.3 \mathrm{~mL})$ at $0{ }^{\circ} \mathrm{C}$. The resulting mixture was stirred at room temperature for 1 hour. The resulting mixture was concentrated under vacuum. The crude product was purified by Prep-HPLC with the following conditions: Column: XBridge Prep OBD C18 Column, 30×150mm 5um; Mobile Phase A: Water (10 mmol/L $\mathrm{NH}_{4} \mathrm{HCO}_{3}$ ), Mobile Phase B: ACN; Flow rate: 60 $\mathrm{mL} / \mathrm{min}$; Gradient: 24 B to $54 \mathrm{~B}$ in $7 \mathrm{~min} ; 254 \mathrm{~nm}$. This resulted in $9.7 \mathrm{mg}$ (39\%) of $\mathrm{N}$-[4-[(E)-2-[4-[(5isopropyl-1H-pyrazol-3-yl)amino]-6-(4-methylpiperazin-1-yl)pyrimidin-2-yl]ethenyl]phenyl]propanamide as a white solid. LCMS (ESI): $[\mathrm{M}+\mathrm{H}]^{+}: 475.4,{ }^{1} \mathrm{H}$ NMR $\left(300 \mathrm{MHz}, \mathrm{CD}_{3} \mathrm{OD}\right) \delta 7.83-7.71(\mathrm{~m}, 1 \mathrm{H}), 7.65-7.50$ $(m, 4 H), 6.95-6.84(m, 1 H), 6.34-6.28(m, 1 H), 6.08-6.02(m, 1 H), 3.70-3.62(m, 4 H), 3.03-2.91(m$, $1 \mathrm{H}), 2.59-2.50(\mathrm{~m}, 4 \mathrm{H}), 2.47-2.32(\mathrm{~m}, 5 \mathrm{H}), 1.31(\mathrm{~d}, J=6.9 \mathrm{~Hz}, 6 \mathrm{H}), 1.25-1.15(\mathrm{~m}, 3 \mathrm{H})$.

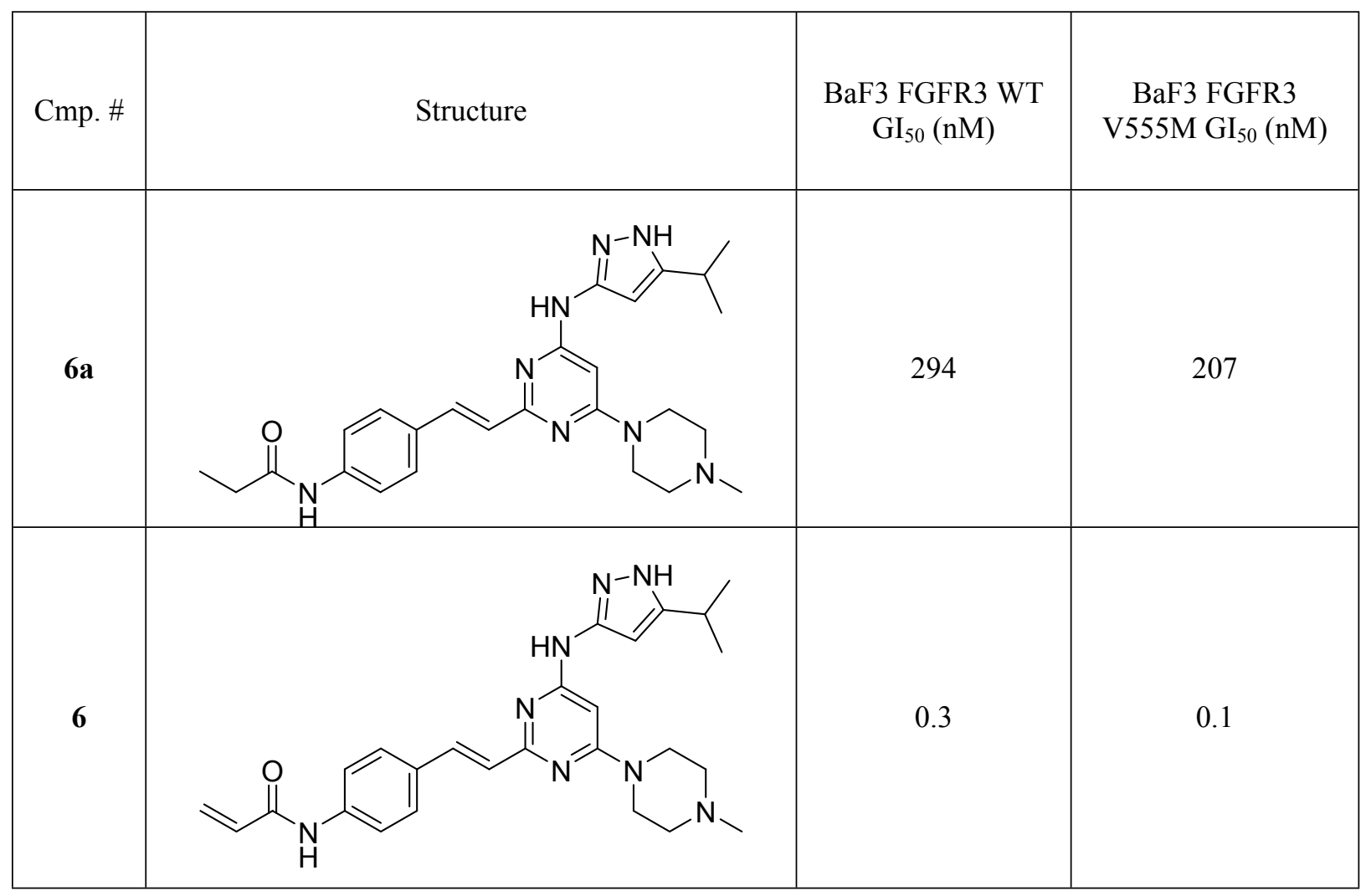

\section{Intact Mass Spectroscopy}


Modified mass detection by LC-MS

FGFR2 (461-768) wild-type was incubated with 2-fold excess of compound to protein (1 $\mu$ M FGFR2) at room temperature for two hours. Mass analyses were carried out on a Thermo Scientific Q-Exactive HRM (ESI source, $4.0 \mathrm{kV}$ ionization voltage, $250^{\circ} \mathrm{C}$ capillary temp., 10 arb sheath gas, S-lens RF level 65) coupled with Accela Open AS 1250. Samples $(10 \mu \mathrm{L})$ were desalted on a C8 column (Agilent Zorbax $300 \mathrm{SB}$ ) with a gradient from $30 \%$ to $80 \%$ B over 5 minutes. Eluent A consisted of $0.1 \%$ formic acid in water and eluent $B$ consisted of $0.1 \%$ formic acid in acetonitrile. The flow was set to $400 \mathrm{uL} / \mathrm{min}$. All solvents were $\mathrm{LC} / \mathrm{MS}$ grade (Thermo Scientific). The mass spectrometer was run in positive mode collecting full scan at $\mathrm{R}=35,000$ from $\mathrm{m} / \mathrm{z} 600$ to $\mathrm{m} / \mathrm{z}$ 2000. Data collected with Xcalibur 3.1 software.

Data analysis

Xcalibur raw files were processed using BioPharma Finder 2.0 (Thermo Scientific) with the ReSpect deconvolution algorithm. Peaks were averaged over the selected retention time to generate source spectra from the TIC chromatogram trace with chromatogram parameters set to $\mathrm{m} / \mathrm{z} 700$ to 2000 . The output from the deconvolution algorithm includes model mass range from 10,000 to $160,000.00$, mass tolerance $20.00 \mathrm{ppm}$, charge state range from 10 to 100 , where the target mass is the estimated mass of protein or protein + compound, with noise rejection of $95 \%$ confidence.

Covalent modification of FGFR2 with Compound 3:

Mass of APO protein: 37009

Mass of Compound 3: 444

Expected mass of covalently modified protein: 37453

Observed mass of covalently modified protein: 37453
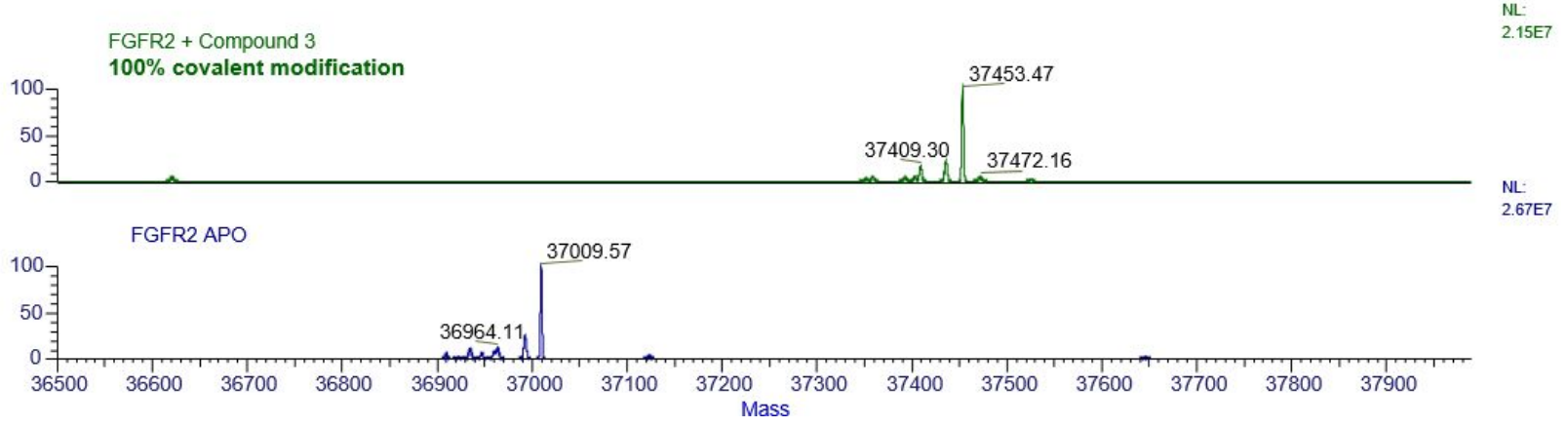

Covalent modification of FGFR2 V564F with Compound 19

Mass of APO protein: 35769

Mass of Compound 19: 486 
Expected mass of covalently modified protein: 36255

Observed mass of covalently modified protein: 36255

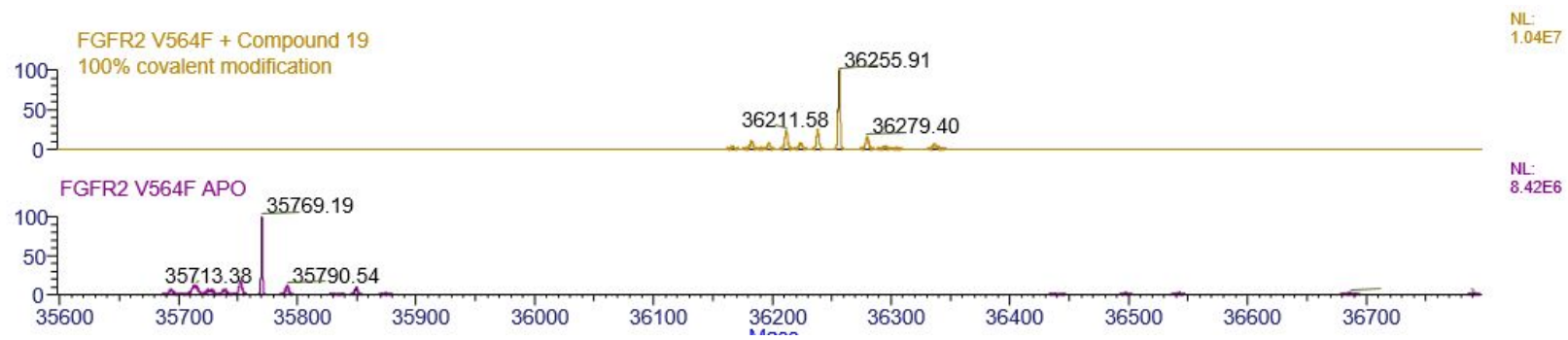

3. Generating FGFR2/3 over-expressing BaF3 cell lines:

\section{Transfection:}

Day 1: Plate $2 \times 10^{6}$ of HEK293FT cells per $10 \mathrm{~cm}$ plate, 1 plate per plasmid

Day 2: Transfection using Mirus method:

Place $1.0 \mathrm{~mL}$ OptiMEM in sterile tube (x4)

In separate tube- prepare packaging mix ( $3 \mu \mathrm{g} \Delta 8.91+3 \mu \mathrm{g}$ VSVG per reaction), add $2 \mu \mathrm{L}$ packaging mix to each sterile tube

\begin{tabular}{|l|l|l|l|l|l|}
\hline Plasmid & Stock $(\mu \mathrm{g} / \mu \mathrm{L})$ & Per reaction $(\mu \mathrm{g})$ & Volume/rxn & \# reactions & Total volume $(\mu \mathrm{L})$ \\
\hline$\Delta 8.91$ & 2.314 & 3 & 1.3 & 4 & 5.19 \\
\hline VSVG & 4.541 & 3 & 0.7 & 4 & 2.64 \\
\hline
\end{tabular}

Add target DNA to appropriate tube, pipet gently to mix

ETV6-FGFR2-WT in pLenti6.3

\begin{tabular}{|l|l|}
\hline Stock $(\mu \mathrm{g} / \mu \mathrm{L}):$ & 0.4 \\
\hline $\begin{array}{l}\text { Vol needed for } 3 \\
\mu \mathrm{g}(\mu \mathrm{L}):\end{array}$ & 7.5 \\
\hline
\end{tabular}

ETV6-FGFR3-WT in pLenti6.3
ETV6-FGFR2-V564F in pLenti6.3

\begin{tabular}{|l|l|}
\hline Stock $(\mu \mathrm{g} / \mu \mathrm{L}):$ & 2 \\
\hline $\begin{array}{l}\text { Vol needed for } 3 \\
\mu \mathrm{g}(\mu \mathrm{L}):\end{array}$ & 1.50 \\
\hline
\end{tabular}

ETV6-FGFR3-V555M in pLenti6.3 


\begin{tabular}{|l|l|}
\hline Stock $(\mu \mathrm{g} / \mu \mathrm{L}):$ & 2 \\
\hline $\begin{array}{l}\text { Vol needed for } 3 \\
\mu \mathrm{g}(\mu \mathrm{L}):\end{array}$ & 1.5 \\
\hline
\end{tabular}

\begin{tabular}{|l|l|}
\hline Stock $(\mu \mathrm{g} / \mu \mathrm{L}):$ & 2.4 \\
\hline $\begin{array}{l}\text { Vol needed for } 3 \\
\mu \mathrm{g}(\mu \mathrm{L}):\end{array}$ & 1.25 \\
\hline
\end{tabular}

Add $30 \mu \mathrm{L}$ TransIT to each tube, pipet gently to mix, incubate at RT for 15 minutes

Add total tube volume to HEK293FT cells in $10 \mathrm{~cm}$ plate

Incubate overnight at $37^{\circ} \mathrm{C}, 5 \% \mathrm{CO}_{2}$

Day 5: Harvest virus

Collect media from $10 \mathrm{~cm}$ plate using sterile syringe

Filter using syringe and put into clean tube

Proceed to infection

Infection:

Day 1:

Seed $2 \times 10^{6} \mathrm{BaF} 3$ cells in $1 \mathrm{~mL}$ RPMI+20\%FBS+ $10 \mathrm{ng} / \mathrm{mL}$ IL-3 in a 6-well plate, one well per viral infection

Add $2 \mathrm{~mL}$ viral supernatant per well, plus $2 \mu \mathrm{L}$ Polybrene

Spin plate 45 mins at $2,500 \mathrm{RPM}, 25^{\circ} \mathrm{C}$

Incubate plate overnight

Day 2:

Spin cells at 1,200 RPM for 5 mins to pellet cells and remove virus

Resuspend cells in $5 \mathrm{~mL}$ complete media and seed into T25 flask to recover

Continue to expand cells. Once a hearty cell population is reached in a T75 culture, begin selection with blasticidin $(20 \mu \mathrm{g} / \mathrm{mL}$ ) for $7-10$ days. Once control flask of parental BaF3 cells is no longer proliferating/ all cells are dead, selection is complete. 


\section{Experimental Procedure for Cellular Viability Assays:}

For viability assay, frozen assay ready BaF3 cells were seeded onto a 384-well plate at 500 cells per well on an assay ready plate that was pre-stamped with compounds in serial dilution. Dose response was measured in 11 point serial dilutions with a top dose of $10 \mu \mathrm{M}$. The percentage of DMSO was controlled throughout and a time zero, DMSO only control was included. Seventy two hours after dosing, CellTiterGlo reagent was added, incubated, and assayed on an EnVision Multilabel Reader (PerkinElmer). Luminescence values from each treatment were normalized to the average value of the respective DMSO control. The dose response curve plots were generated in GraphPad Prism and $\mathrm{Gl}_{50}$ 's calculated from nonlinear regression analysis. All $\mathrm{Gl}_{50}$ values are reported as a mean of a minimum of 2 runs, with standard deviations typically less than $25 \%$ of the $\mathrm{Gl}_{50}$ value. Runs with higher variability were repeated to lower standard deviation.

\section{Preparation of Proteins for TR-FRET Assays:}

\section{FGFR2 V564F:}

His-FGFR2 V564F was expressed in insect cells. A 2.7L expression culture was harvested after $48 \mathrm{hrs}$. Clarified lysate was prepared by sonication and centrifugation and loaded onto a $2 \mathrm{ml} \mathrm{HisTrap} \mathrm{column} \mathrm{in} \mathrm{50mM} \mathrm{Tris} \mathrm{pH} \mathrm{8.0,} 300$ $\mathrm{mM} \mathrm{NaCl}, 10 \mathrm{mM}$ imidazole and $1 \mathrm{mM}$ TCEP and eluted with 20 column volume of the same buffer with 10-500 mM imidazole gradient. Fractions containing His-FGFR2 V564F were pooled and loaded onto S300 (16/60) column preequilibrated with $20 \mathrm{mM}$ Tris pH8.0, $20 \mathrm{mM} \mathrm{NaCl} \& 2 \mathrm{mM}$ TCEP. The elution peak was concentrated to $1.5 \mathrm{mg} / \mathrm{mL}$ and frozen at -80 .

\section{HisTrap}

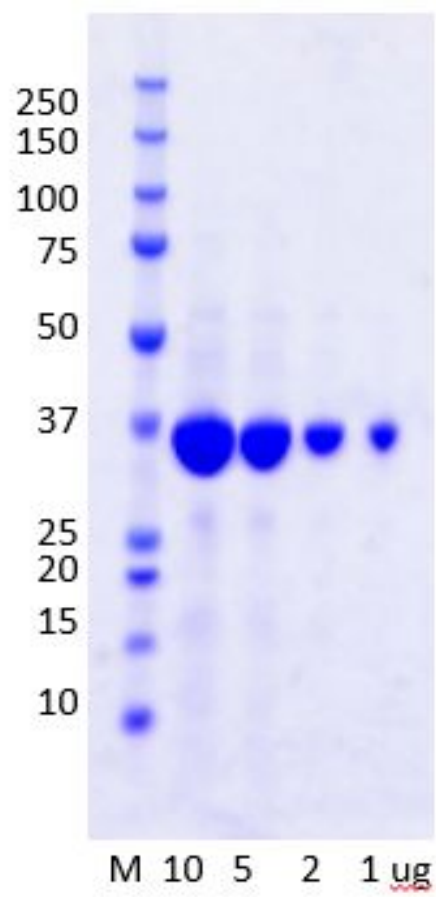


Protein sequence:

MAHHHHHHEN LYFQGAGVSE YELPEDPKWE FPRDKLTLGK PLGEGCFGQV VMAEAVGIDK DKPKEAVTVA VKMLKDDATE KDLSDLVSEM EMMKMIGKHK NIINLLGACT QDGPLYVIFE YASKGNLREY LRARRPPGME YSYDINRVPE EQMTFKDLVS CTYQLARGME YLASQKCIHR DLAARNVLVT ENNVMKIADF GLARDINNID YYKKTTNGRL PVKWMAPEAL FDRVYTHQSD VWSFGVLMWE IFTLGGSPYP GIPVEELFKL LKEGHRMDKP ANCTNELYMM MRDCWHAVPS QRPTFKQLVE DLDRILTLTT NEE

\section{FGFR3 V555M:}

FGFR3 V555M was expressed in insect cells. A 2.7L expression culture was harvested after 48 hrs. Clarified lysate was prepared by sonication and centrifugation and loaded onto a $2 \mathrm{ml} \mathrm{HisTrap} \mathrm{column} \mathrm{in} 50 \mathrm{mM}$ Tris $\mathrm{pH} 8.0,300$ $\mathrm{mM} \mathrm{NaCl}, 10 \mathrm{mM}$ imidazole and 1mM TCEP and eluted with 20 column volume of the same buffer with 10-500 mM imidazole gradient. Fractions containing His-FGFR3 V555M were pooled and subjected to TEV proteolysis to remove the amino-terminal His6 tag, while dialyzing against $20 \mathrm{mM}$ Tris $\mathrm{pH} 8.0,20 \mathrm{mM} \mathrm{NaCl}$ \& $1 \mathrm{mM}$ TCEP. Protein was subjected to a second HisTrap column and the tag-cleaved protein was collected in the flow through. This was then loaded onto S300 (16/60) column pre-equilibrated with $20 \mathrm{mM}$ Tris pH8.0, $20 \mathrm{mM} \mathrm{NaCl} \& 2 \mathrm{mM}$ TCEP. The elution peak was concentrated to $18.8 \mathrm{mg} / \mathrm{mL}$ and frozen at -80 . 


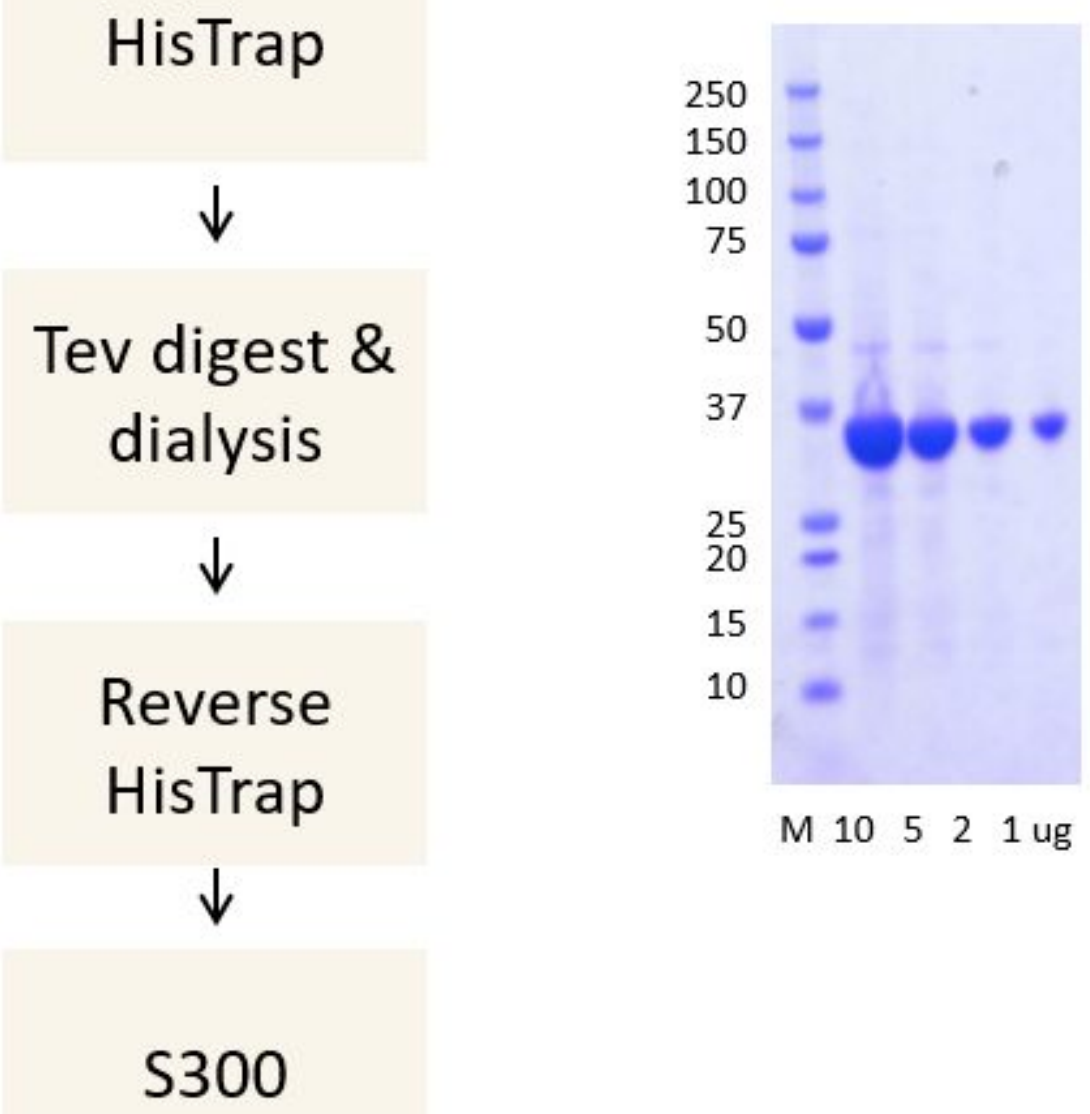

Protein sequence:

MAHHHHHHEN LYFQGANVSE LELPADPKWE LSRARLTLGK PLGEGCFGQV VMAEAIGIDK DRAAKPVTVA VKMLKDDATD KDLSDLVSEM EMMKMIGKHK NIINLLGACT QGGPLYVLME YAAKGNLREF LRARRPPGLD YSFDTSKPPE EQLTFKDLVS CAYQVARGME YLASQKCIHR DLAARNVLVT EDNVMKIADF GLARDVHNLD YYKKTTNGRL PVKWMAPEAL FDRVYTHQSD VWSFGVLLWE IFTLGGSPYP GIPVEELFKL LKEGHRMDKP ANCTHDLYMI MRECWHAAPS QRPTFKQLVE DLDRVLTVTS TDE

\section{FGFR TR-FRET Activity Assay:}

Inhibition of FGFR enzymatic activity was measured in a TR-FRET assay, monitoring phosphorylation of a poly Glu-Tyr peptide substrate. $5 \mu \mathrm{L}$ of purified FGFR protein diluted in assay buffer ( $20 \mathrm{mM}$ HEPES $\mathrm{pH} 7.5,10 \mathrm{mM} \mathrm{MgCl}$, 1 mM EGTA, 2 mM DTT, 0.002\% BSA and 0.02\% Brij 35) was added to a 384-well plate (Corning, Cat \# 3820) containing inhibitor compounds pre-dispensed with an acoustic dispenser. 
The final concentration of FGFR protein was as follows: 500 pM WT FGFR3, 20 pM FGFR2-V564F or 20 pM FGFR3-V555M. FGFR protein was preincubated with inhibitor for $1 \mathrm{hr}$ at room temperature prior to the addition of $5 \mu \mathrm{L}$ of ATP and poly Glu-Tyr (Perkin Elmer Cat\# TRF0100-D) substrates, at final concentrations of $50 \mu \mathrm{M}$ and $100 \mathrm{nM}$, respectively. After $1 \mathrm{hr}$ the reaction was stopped by the addition of $10 \mu \mathrm{L}$ of detection buffer (Perkin Elmer Cat\# CR97-100) containing 20 mM EDTA and $2 \mathrm{nM}$ Lance EuW1024-antiphosphoTyrosine (PT66) (Perkin Elmer cat\# AD0069). After 30 minutes, TR-FRET signal was measured on an Envision plate reader using a $320 \mathrm{~nm}$ excitation filter and a $665 \mathrm{~nm}$ emission filter.

\section{High Throughput Screen:}

FGFR3-V555M was screened in the TR-FRET assay against a library of $10 \mathrm{~K}$ compounds enriched for hinge binding motifs at a compound concentration of $10 \mu \mathrm{M}$. FGFR protein was preincubated with compound for $1 \mathrm{hr}$ at room temperature prior to initiating the reaction with $50 \mu \mathrm{M}$ ATP and $100 \mathrm{nM}$ poly Glu-Tyr substrates. The reactions were stopped and TR-FRET measured as described above. Any compounds inhibiting FGFR activity greater than 3 standard deviations from the mean were selected as hits. Hits were then confirmed and potency determined in an 11-point dose response curve.

\section{Procedures for DMPK/Physiochemical Assays:}

\section{Blood stability assay:}

Human blood was obtained from Healthy Asian volunteers from local vendor, collected in domestic hospitals with Ethical approval. Blood stability was assessed by incubating test compound or positive control (Propantheline) at a final concentration of $2 \mu \mathrm{M}$ in whole blood (500 $\mu \mathrm{L}, 0.5 \%$ final solvent concentration). The assay are performed in duplicate. Incubate the reaction samples at $37^{\circ} \mathrm{C}$ in a water bath with shaking at approximately $60 \mathrm{rpm}$. Take aliquots of $50 \mu \mathrm{L}$ from the reaction samples at $0,15,30$, 45,60 and 120 minutes. Stop the reaction by adding $350 \mu \mathrm{L}$ of room temperature quench solution (Acetonitrile containing internal standards (IS, $100 \mathrm{nM}$ alprazolam, $200 \mathrm{nM}$ labetalol, $200 \mathrm{nM}$ Imipramine and $2 \mu \mathrm{M}$ ketoplofen)). Vortex for 5 minutes. Centrifuge samples in plate at 3,220 g for 30 minutes at room temperature to precipitate protein. Transfer $100 \mu \mathrm{L}$ of the supernatant to a new plate. The supernatant may be diluted with $100 \mu \mathrm{L}$ or $200 \mu \mathrm{L}$ water according to the LC/MS signal response and peak shape. Mix well and analyze samples using LC/MS/MS. For data analysis, all calculations are carried out using Microsoft Excel. Percent parent compounds remaining at each time point will be estimated by determining the peak area ratios from extracted ion chromatograms. In vitro $t_{1 / 2}$ values were determined by plotting the natural logarithm of the analyte/IS peak area ratios as a function of time, with the slope of the linear regression $(-k)$ converted to in vitro $t_{1 / 2}$ value, where $t_{1 / 2}=-0.693 / k$.

Hepatocyte metabolic stability assay:Pooled cryopreserved Human hepatocytes (10-Donor pooled) were purchased from BiolVT. Cells where quickly thawed and re-suspended in Williams E media supplemented with glutamax at a final density of $0.5 \times 10^{6}$ viable cells $/ \mathrm{mL}$. Cells were transferred in a 96 well plate and pre-incubated for 10 minutes at $37^{\circ} \mathrm{C}$. The reaction is initiated by adding the test compound at a final concentration of $1 \mu \mathrm{M}$ (final DMSO concentration $0.5 \%$ ). Negative control incubations consists of boiled hepatocytes $\left(0.5 \times 10^{6}\right.$ viable cells $\left./ \mathrm{mL}\right)$ also incubated with $1 \mu \mathrm{M}$ test compound. Positive control used was 
verapamil at a $1 \mu \mathrm{M}$ final concentration. Each compound was incubated for $0,15,30,60,90$ and $120 \mathrm{~min}$. The negative and positive controls were also incubated for the same period of time. The reactions were stopped by transferring $25 \mu \mathrm{L}$ from the respective time points with the addition of 6 volumes of cold acetonitrile containing internal standards (IS: 200 nM Caffeine, 200 nM labetalol, $200 \mathrm{nM}$ diclofenac and $100 \mathrm{nM}$ alprazolam) to terminate the reactions. The 96 well plate was then centrifuged for 25 minutes at $3,220 \mathrm{~g}$. Aliquots of $100 \mu \mathrm{L}$ of the supernatants are then mixed with $100 \mu \mathrm{L}$ ultrapure water and used for $\mathrm{LC} / \mathrm{MS} / \mathrm{MS}$ analysis. In vitro $t_{1 / 2}$ values were determined by plotting the natural logarithm of the analyte/IS peak area ratios as a function of time, with the slope of the linear regression $(-k)$ converted to in vitro $t_{1 / 2}$ value, where $t_{1 / 2}=-0.693 / k$. Subsequently, $\mathrm{CL}_{\text {int }}$ were calculated as: (incubation volume/number of cells) $x$ 0.693/ $t_{1 / 2}$. Subsequently, scaled $C L$ values were calculated using the well-stirred venous equation model (Houston, 1994; Pang \& Rowland, 1977).

\section{Caco-2 Cell Culture and Permeability Studies:}

Caco2 cells from American Type Culture Collection (Rockville, MD) were routinely maintained in Dulbecco's modified Eagle's medium (DMEM, Gibco Life Technologies, Carlsbad, CA) containing 10\% fetal bovine serum, with high glucose and L-glutamine supplemented with: 10\% FBS, 1X penicillin-streptomycin mixture and $1 \mathrm{X}$ non-essential amino acids (NEAA). Cells were grown in T-75 flask in an incubator in an atmosphere of $5 \% \mathrm{CO} 2$ and $95 \%$ relative humidity at $37^{\circ} \mathrm{C}$. Allow cells to reach $80-90 \%$ confluence before detaching and splitting. Cells are then trypsinized, centrifuged at $120 \mathrm{~g}$ for $10 \mathrm{~min}$ and resuspended in seeding medium at a density of $6.86 \times 10^{5}$ cells $/ \mathrm{mL}$. This cell concentration can be used to seed $2.40 \times 10^{5}$ cells $/ \mathrm{cm}^{2} .50 \mu \mathrm{L}$ of this cell suspension is then seeded in 96 well Transwell plate (96-well Transwell plate,

Corning Costar Co., Corning, NY). The cells on the inserts were cultured for 21 days at $37^{\circ} \mathrm{C}$ in a humidified incubator containing 5\% CO2 in air. The differentiation status of the formed monolayer was evaluated by measuring the transepithelial electrical resistance (TEER). Permeability studies were conducted with the monolayers that developed TEER values $>300 \Omega \mathrm{cm} 2$ following 21 days in cell culture.

On the day of the experiment, DMEM was removed, and the monolayers were rinsed two times using prewarmed HBSS containing $10 \mathrm{mM} \mathrm{HEPES,} \mathrm{pH} \mathrm{7.4(Transport} \mathrm{buffer).} \mathrm{Then} \mathrm{incubated} \mathrm{with} \mathrm{the} \mathrm{same} \mathrm{media}$ for 30 minutes at $37{ }^{\circ} \mathrm{C}$. To assess A-B permeability, the blank transport buffer was removed from the apical compartment and replaced with $75 \mu \mathrm{L}$ of transport buffer containing $5 \mu \mathrm{M}$ of test compound or controls (Digoxin, propranolol and Prazosin). The final concentration of DMSO in the incubation system is less than $0.1 \%$. On the basolateral compartment, $235 \mu \mathrm{L}$ of transport buffer was added. To determine the rate of drug transport from the basolateral to apical direction. Add $235 \mu \mathrm{L}$ of transport buffer containing $5 \mu \mathrm{M}$ of test compound or controls (as stated above). After $2 \mathrm{hrs}$ of incubation, remove $50 \mu \mathrm{L}$ directly from the apical and basolateral wells (using the basolateral access holes) and transfer to a new pate. Then add 4 volume of cold methanol containing appropriate internal standards (100 nM alprazolam, $200 \mathrm{nM}$ labetalol, $200 \mathrm{nM}$ caffeine and $200 \mathrm{nM}$ diclofenac) to terminate the reaction. Vortex for 5 minutes. Samples are centrifuged at $3,220 \mathrm{~g}$ for 30 minutes. An aliquot of $100 \mu \mathrm{L}$ of the supernatant was then mixed with $100 \mu \mathrm{L}$ of ultra-pure water. The samples were analyzed by LC-MS/MS. 
For data analysis, all calculations were carried out using Microsoft Excel. Peak areas were determined from extracted ion chromatograms. The apparent permeability coefficient (Papp), in units of centimeter per second, can be calculated for Caco-2 drug transport assays using the following equation:

$$
P_{a p p}=\frac{V_{A}}{\text { Area } \times \text { time }} \times \frac{[d r u g]_{a c c e p t o r}}{[d r u g]_{\text {initial ,donor }}}
$$

Where $\mathrm{VA}$ is the volume (in $\mathrm{mL}$ ) in the receiver well $(0.235 \mathrm{~mL}$ for $\mathrm{Ap} \rightarrow \mathrm{BI}$ flux and $0.075 \mathrm{~mL}$ for $\mathrm{BI} \rightarrow \mathrm{Ap}$ flux), Area is the surface area of the membrane $(0.143 \mathrm{~cm} 2$ for HTS Transwell-96 Well Permeable Supports), and time is the total transport time in seconds.

The efflux ratio can be determined using the following equation:

$$
\text { Efflux Ratio }=\frac{P_{a p p(B-A)}}{P_{a p p(A-B)}}
$$

Where Papp (B-A) indicates the apparent permeability coefficient in basolateral to apical direction, and Papp (A-B) indicates the apparent permeability coefficient in apical to basolateral direction.

\section{Liver microsome metabolic stability assay:}

Pooled Human liver microsomes (mixed gender) were purchased from Corning Life Sciences (Amsterdam, the Netherlands). CD-1 Mouse liver microsomes were purchased from Xenotech. Liver microsomes $(0.5 \mathrm{mg} / \mathrm{mL})$ were pre-incubated 5 minutes at $37^{\circ} \mathrm{C}$ in the following mixture, $0.1 \mathrm{M}$ phosphate buffer $\mathrm{pH}$ 7.4 containing $5 \mathrm{mM} \mathrm{MgCL}_{2}$ and $1 \mathrm{mM} \mathrm{NADPH}$. The reaction is initiated by adding the test compound at a final concentration of $2 \mu \mathrm{M}$ (final DMSO concentration $0.5 \%$ ). Negative control incubations were included where phosphate buffer was added instead of NADPH. Positive control used was verapamil for both Human and Mouse liver microsomes. Each compound was incubated for 0, 15, 30, 45 and 60 min. The negative and positive controls were also incubated for the same period of time. The reactions were stopped by transferring $50 \mu \mathrm{L}$ from the reaction solution at $0,15,30,45$ and 60 minutes with the addition of 4 volumes of cold acetonitrile $0.1 \%$ formic acid- with internal standard(IS) (100 nM alprazolam, $200 \mathrm{nM}$ imipramine, $200 \mathrm{nM}$ labetalol and $2 \mu \mathrm{M}$ ketoprofen). Samples where then centrifuged at 3,220 g for 40 minutes at $4{ }^{\circ} \mathrm{C}$ to precipitate the proteins. Then $90 \mu \mathrm{L}$ of the supernatant was transferred to each well of a new 96-well plate containing an appropriate volume of ultra-pure water (depends on the LC-MS/MS signal response and peak shape) for LC-MS/MS analysis. In vitro $t_{1 / 2}$ values were determined by plotting the natural logarithm of the analyte/IS peak area ratios as a function of time, with the slope of the linear regression $(-k)$ converted to in vitro $t_{1 / 2}$ value, where $t_{1 / 2}=-0.693 / k$. Subsequently, intrinsic $C L\left(C L_{\text {int }}\right)$ was calculated as: (incubation volume/microsomal protein) $\times 0.693 / t_{1 / 2}$ and scaled $\mathrm{CL}$ values were obtained using the well-stirred venous equilibration model (Houston, 1994; Pang \& Rowland, 1977). 


\section{Solubility analysis:}

\section{Preparation of Stock Solutions}

The stock solutions of test compounds were prepared in DMSO at the concentration of $10 \mathrm{mM}$, and the stock solution of control compound was prepared in DMSO at the concentration of $30 \mathrm{mM}$. Progesterone was used as positive control in the assay.

\section{Procedures for Solubility Determination}

$30 \mu \mathrm{L}$ stock solution of each compound was placed in order into their proper 96 -well rack, followed by adding $970 \mu \mathrm{L}$ of 100uM Phosphate buffer at pH 7.4 into each vial of the cap-less Solubility Sample plate. This study was performed in duplicate. One stir stick was added to each vial and then vials were sealed using a molded PTDE/SIL 96-Well Plate Cover. The Solubility Sample plate was transferred to the Thermomixer Comfort plate shaker and incubated at RT for 2 hours and 24 hours with shaking at 1100 rpm. After 2 hours and 24 hours incubation, stir sticks were removed using a big magnet and all samples from the Solubility Sample plate were transferred into the filter plate. All the samples were filtered by using the Vacuum Manifold. The filtered samples were diluted with methanol. The dilution factor might be changed according to the solubility value and the LC/MS signal response.

3. Preparation of $0.3 \mu \mathrm{M}$ Standards (progesterone) (STD)

$10 \mathrm{mM}$ DMSO compound stock solutions were diluted with DMSO to $300 \mu \mathrm{M}$ and then diluted with methanol to obtain $0.3 \mu \mathrm{M}$ STDs.

\section{Sample Analysis}

Samples were analyzed by LC-MS/MS.

\section{Data Analysis}

All calculations were carried out using Microsoft Excel.

The solution filtered was analyzed and quantified against a standard of known concentration in DMSO using LC coupled with Mass spectral peak identification and quantitation. The solubility values of the test compounds were calculated as follows:

$$
[\text { Sample }]=\frac{A R E A_{\text {Sample }} \times I N J V O L_{\text {Std }} \times D F_{\text {Sample }} \times[S T D]}{A R E A_{\text {Std }} \times I N J \text { VOL }}
$$

DF means the dilution factor 


\section{Bioanalytical Method}

\section{Chromatographic conditions}

LC system: Shimadzu

MS analysis: API 4000 instrument from AB Inc. (Canada) with an ESI interface

Column: Phenomenex Synergi $4 \mu$ Hydro-PR 80A $(2.0 \times 30 \mathrm{~mm})$ coupled with preguard column

Mobile phase: $0.1 \%$ formic acid in acetonitrile $(\mathrm{A})$ and

$0.1 \%$ formic acid in water $(B$

\begin{tabular}{lllllll}
\hline Time $(\min )$ & 0 & 0.3 & 0.8 & 1.1 & 1.2 & 1.4 \\
\hline \% A & 5 & 5 & 100 & 100 & 5 & 5 \\
\hline
\end{tabular}

Elution rate: $0.65 \mathrm{~mL} / \mathrm{min}$

Column temperature: $40^{\circ} \mathrm{C}$

Injection volume: $10 \mu \mathrm{L}$

\section{MS parameters}

Ion source: Turbo spray

Ionization model: ESI

Scan type: MRM

Collision gas: $6 \mathrm{~L} / \mathrm{min}$

Curtain gas: $30 \mathrm{~L} / \mathrm{min}$

Nebulize gas: $50 \mathrm{~L} / \mathrm{min}$

Auxiliary gas: $50 \mathrm{~L} / \mathrm{min}$

Temperature: $500^{\circ} \mathrm{C}$

lonspray voltage: +5500 v (positive MRM) 


\section{Pharmacokinetics:}

Male CD-1 mice were dosed with H3B compound intravenously (IV group, $\mathrm{n}=3$ ) at $5 \mathrm{mg} / \mathrm{kg}$ and orally (PO group, $\mathrm{n}=3$ ) at $10 \mathrm{mg} / \mathrm{kg}$, after fasting overnight and through 4 hours post dose (free access to water and food in our standard PK studies). Animals were weighed prior to dose administration. IV formulation consisted of $\mathrm{H} 3 \mathrm{~B}$ compound in the described formulation, whereas $\mathrm{H} 3 \mathrm{~B}$ compound was formulated in $0.5 \%(\mathrm{w} / \mathrm{v})$ methylcellulose with $0.1 \%(\mathrm{v} / \mathrm{v})$ Tween $^{\circledR} 80$ in water for PO dosing. Serial blood samples were collected via the Dorsal metatarsal vein, at 0.0833 (IV group only), 0.25, 0.5, 1, 2, 4, 8 and 24 hours after dosing. Blood samples were collected into K2-EDTA tubes and placed on wet ice until centrifuged (approximately $4000 \mathrm{~g}$ for $5 \mathrm{~min}$ at $4^{\circ} \mathrm{C}$ ) within 30 minutes of collection. Plasma and dose formulation samples, stored at below $-75^{\circ} \mathrm{C}$, were analyzed for $\mathrm{H} 3 \mathrm{~B}$ compound using a qualified LC-MS/MS method, with a lower limit of quantitation (LLOQ) of $0.5 \mathrm{ng} / \mathrm{mL}$.

PK parameters were determined using individual plasma concentration versus time profiles using Phoenix WinNonlin (Version 8.0, Certara, Princeton, NJ). Standard non-compartmental PK methods were used to calculate the area under the plasma concentration-time curve (AUC) using the linear trapezoidal method. The acceptance criteria for terminal half-life determination include regression of at least three time points in the elimination phase and $r 2>0.9$. Nominal sample times were used in the PK analysis.

\section{LC-MS/MS System}

Instrument: Prominence (Degasser DGU-20A5R(C), Serial NO. L20705619752 IX; Liquid Chromatograph LC-30AD Serial NO. L20555611120 AE and L20555611116 AE;Communications Bus Module CBM-20A, Serial NO. L20235635001 CD, Auto SIL-20AC HT, Serial No. L20355305356 AE; Rack changer II, Serial No. L20585300757 SS)

MS AB Sciex Triple Quan 5500 LC/MS/MS instrument (Serial NO. EF20711807)

Column HALO 90Å, C18, $2.7 \mu \mathrm{m}, 2.1 * 50 \mathrm{~mm}, \quad$ SN : USKL004028

\section{HPLC Conditions:}

\section{Mobile Phase}

Solution A: 5\% Acetonitrile in Water (0.1\% Formic acid)

Solution B: 95\% Acetonitrile in Water (0.1\% Formic acid)

\section{Gradient:}

Flow rate: $0.5 \mathrm{~mL} / \mathrm{min}$

$\begin{array}{lll}\text { Time }(\min ) & \text { A (\%) } & \text { B (\%) }\end{array}$




\begin{tabular}{lll}
0.20 & 95.0 & 5.00 \\
1.50 & 5.00 & 95.0 \\
2.00 & 5.00 & 95.0 \\
2.01 & 95.0 & 5.00 \\
2.50 & 95.0 & 5.00 \\
\hline
\end{tabular}

\section{X-Ray Crystal Structure Determination and Data}

\section{FGFR2 $^{\mathrm{V} 564 \mathrm{~F}}$ protein expression and purification}

Construct encoding residues 458-768 of the FGFR2 kinase domain with the gate-keeper mutation V564F was cloned into the expression vector pET28a that also contains His6 tag at the $\mathrm{N}$-terminus. A TEV cleavage site was engineered between His6 tag and FGFR2 to aid in purification. His6-FGFR2 ${ }^{\mathrm{V} 564 \mathrm{~F}}$ protein was expressed in E. coli BL21(DE3) cells. When cell density reached an OD600 of $\sim 1.0$, protein expression was induced with $0.3 \mathrm{mM}$ IPTG at $18{ }^{\circ} \mathrm{C}$ overnight. Cells were harvested and lysed by sonication. His6-FGFR2 ${ }^{\mathrm{V} 564 \mathrm{~F}}$ was purified from the cleared bacteria cell lysate by Ni affinity chromatography (GE Healthcare). Then His6 tagged TEV protease at 1:10 molar ratio was used to digest Ni column purified His6-FGFR2 ${ }^{\text {V564F }}$ during dialysis against $25 \mathrm{mM}$ Tris, $\mathrm{pH} 7.6,0.15 \mathrm{M} \mathrm{NaCl}, 1 \mathrm{mM}$ 2-mercaptoethanol at $4{ }^{\circ} \mathrm{C}$ overnight. After that, the digested FGFR2 ${ }^{\mathrm{V} 564 \mathrm{~F}}$ sample was further purified by a second $\mathrm{Ni}$ affinity chromatography to remove cleaved His6 tag, followed by a HiPrep ${ }^{\mathrm{TM}}$ 16/60 Sephacryl® S-300 HR size exclusion chromatography (GE Healthcare). The FGFR2 ${ }^{\mathrm{V} 564 \mathrm{~F}}$ protein was concentrated to $\sim 50 \mathrm{mg} / \mathrm{ml}$ in size buffer ( $25 \mathrm{mM}$ Tris-HCl, $\mathrm{pH} 7.6,50 \mathrm{mM} \mathrm{NaCl}, 2 \mathrm{mM}$ TCEP), aliquoted and frozen at $-80^{\circ} \mathrm{C}$. All the purification steps were performed at $4{ }^{\circ} \mathrm{C}$. Proteins concentration was quantified by using the Bradford assay (ThermoFisher Scientific) for crude samples and by OD280 reading using a nanodrop instrument (ThermoFisher Scientific) for the final purified sample. Protein purity was examined by $4-20 \%$ gradient SDS-PAGE.

\section{Crystallization and data collection}


The apo FGFR2 ${ }^{\mathrm{V} 564 \mathrm{~F}}$ crystals were grown by hanging drop vapor diffusion method in the condition with reservoir solution containing 12-15\% PEG4000, 0.1M HEPES, pH 7.5, 0.2 M Ammonium sulfate according to literature report [1]. For crystallization of protein and compound complex, the FGFR2 ${ }^{\mathrm{V} 564 \mathrm{~F}}$ protein at $16 \mathrm{mg} / \mathrm{mL}$ was mixed with the covalent compound 3 or 19 at a molar ratio of 1:2, incubated at room temp for 4 h. Preliminary crystallization screening was performed using various commercial crystallization screen kits (Hampton Research) by sitting drop vapor diffusion method at room temperature. After optimization, the diffraction quality plate-shaped crystals were grown in hanging drops by mixing equal volumes of protein complex with the reservoir solution at room temperature. FGFR2 ${ }^{\mathrm{V} 564 \mathrm{~F}}$ and compound 3 complex was crystallized using the reservoir solution $15 \% \mathrm{w} / \mathrm{v}$ PEG 8000, 0.1M sodium citrate, $\mathrm{pH} 4.0$. FGFR2 ${ }^{\mathrm{V} 564 \mathrm{~F}}$ and compound 19 complex was crystallized using the reservoir solution $12 \% \mathrm{w} / \mathrm{v}$ PEG 8000, 0.1M sodium citrate, $\mathrm{pH} 4.0$. For data collection, crystals were transferred to the reservoir solution plus $20 \%(\mathrm{v} / \mathrm{v})$ glycerol as a cryoprotectant before flash-freezing in liquid nitrogen. The X-ray diffraction data were collected at $100 \mathrm{~K}$ on the synchrotron beam line NSLS-II 17-ID AMX.

\section{Structure determination and refinement}

Diffraction data were processed using $X D S$ program [2]. The FGFR2 and compound complex structures were solved by molecular replacement method using FGFR2 structure (PDB ID: 3RI1) as a search model. Refinement of the initial model were performed using CCP4 [3]. Iterative rounds of manual model building were carried out in COOT [4]. The $R_{\text {free }}$ value was determined on the subset of $5.0 \%$ of the total reflections. The final model for FGFR2 ${ }^{\mathrm{V} 564 \mathrm{~F}}$ and compound 3 was refined to an $\mathrm{R}$ factor of 0.202 and an $R_{\text {free }}$ factor of 0.229 . The final model for FGFR2 ${ }^{\mathrm{V} 564 \mathrm{~F}}$ and compound 19 was refined to an R factor of 0.201 and an $R_{\text {free }}$ factor of 0.224 . The final diffraction data and refinement statistics were shown in supplemental table 1. Structural figures were prepared using the $P y M O L$ software[5].

PDB codes:

FGFR2 V564F with Compound 3: 7KIE

FGFR2 V564F with Compound 19: 7KIA 
1. Eathiraj, S., et al., A novel mode of protein kinase inhibition exploiting hydrophobic motifs of autoinhibited kinases: discovery of ATP-independent inhibitors of fibroblast growth factor receptor. The Journal of biological chemistry, 2011. 286(23): p. 20677-87.

2. Kabsch, W., Xds. Acta crystallographica. Section D, Biological crystallography, 2010. 66(Pt 2): p. 125-32.

3. Winn, M.D., et al., Overview of the CCP4 suite and current developments. Acta Crystallogr D Biol Crystallogr, 2011. 67(Pt 4): p. 235-42.

4. Emsley, P. and K. Cowtan, Coot: model-building tools for molecular graphics. Acta Crystallogr D Biol Crystallogr, 2004. 60(Pt 12 Pt 1): p. 2126-32.

5. DeLano, W.L. and J.W. Lam, PyMOL: A communications tool for computational models. Abstr Pap Am Chem S., 2005. 230: p. U1371-U1372.

Supplemental Table 1 Data collection and refinement statistics

\begin{tabular}{|c|c|c|}
\hline & FGFR2V564F_Compound3 & FGFR2V564F_Compound19 \\
\hline Space group & $\mathrm{P} 2{ }_{1} 2_{1} 2$ & $\mathrm{P} 2{ }_{1} 2_{1} 2$ \\
\hline \multicolumn{3}{|l|}{ Cell dimensions } \\
\hline$a, b, c(\AA)$ & $106.3,116.9,64.7$ & $106.0,117.3,63.8$ \\
\hline$\alpha, \beta, \gamma\left(^{\circ}\right)$ & $90,90,90$ & $90,90,90$ \\
\hline Resolution $(\AA ̊)$ & $56.6-2.47(2.53-2.47)$ & $58.7-2.22(2.28-2.22)$ \\
\hline$R_{\text {sym }}(\%)$ & $14.3(113)^{*}$ & $11.7(116)^{*}$ \\
\hline$R_{p i m}(\%)$ & $8.4(67.8)^{*}$ & $6.8(66.2)^{*}$ \\
\hline$C C_{1 / 2}(\%)$ & $99.7(59.5)^{*}$ & $99.7(63.6)^{*}$ \\
\hline$I / \sigma I$ & $10.4(1.6)^{*}$ & $9.8(1.7)^{*}$ \\
\hline Completeness (\%) & $100(99.9)^{*}$ & $100(100)^{*}$ \\
\hline Redundancy & $7.3(7.2)^{*}$ & $7.4(7.8)^{*}$ \\
\hline Refinement & & \\
\hline
\end{tabular}




\begin{tabular}{|c|c|c|}
\hline Resolution $(\AA)$ & $50-2.47$ & $50-2.22$ \\
\hline No. reflections (test set) & $28141(1471)$ & $38036(2032)$ \\
\hline$R_{\text {work }} / R_{\text {free }}(\%)$ & $20.2 / 22.9$ & $20.1 / 22.4$ \\
\hline \multicolumn{3}{|l|}{ No. atoms } \\
\hline Protein & 4680 & 4631 \\
\hline Ligand & 104 & 100 \\
\hline Water & 153 & 207 \\
\hline \multicolumn{3}{|l|}{ B-factors $\left(\AA^{2}\right)$} \\
\hline Protein & 52.5 & 47.9 \\
\hline Ligand/ion & 72.6 & 58.8 \\
\hline Water & 47.2 & 47.6 \\
\hline \multicolumn{3}{|l|}{ R.m.s deviations } \\
\hline Bond lengths $(\AA)$ & 0.005 & 0.005 \\
\hline Bond angles $\left({ }^{\circ}\right)$ & 1.41 & 1.30 \\
\hline \multicolumn{3}{|c|}{ Ramachandran plot statistics (\%) } \\
\hline Favored & 95.6 & 96.6 \\
\hline Allowed & 4.4 & 3.0 \\
\hline Disallowed & 0.0 & 0.4 \\
\hline
\end{tabular}

Diffraction data were collected from a single crystal.

$5 \%$ randomly selected reflections were used as a test set.

*Highest resolution shell is shown in parenthesis.

\section{Kinase Selectivity Data}

A selection of compounds were screened in a mini-kinase panel from DiscoverX. The panel consisted of the 4 isoforms of FGFR, other kinases with a p-loop cysteine, and kinases with reported off-target activity in other FGFR programs. Here are the experimental details and assay results:

\section{Tech nology Overview}

The KINOMEscan ${ }^{\mathrm{TM}}$ screening platform employs a novel and proprietary active site-directed competition binding assay to quantitatively measure interactions between test compounds and more than 450 human kinases and disease relevant mutant variants. This robust and reliable assay technology affords investigators the ability to extensively annotate compounds with accurate, precise and reproducible data. 
KINOMEscan ${ }^{\mathrm{TM}}$ assays do not require ATP and thereby report true thermodynamic interaction affinities, as opposed to IC50 values, which can depend on the ATP concentration.

\section{How KINOMEscan ${ }^{\mathrm{TM}}$ Works}

Compounds that bind the kinase active site and directly (sterically) or indirectly (allosterically) prevent kinase binding to the immobilized ligand, will reduce the amount of kinase captured on the solid support (A \& B). Conversely, test molecules that do not bind the kinase have no effect on the amount of kinase captured on the solid support $(C)$. Screening "hits" are identified by measuring the amount of kinase captured in test versus control samples by using a quantitative, precise and ultra-sensitive qPCR method that detects the associated DNA label (D). In a similar manner, dissociation constants (Kds) for test compound-kinase interactions are calculated by measuring the amount of kinase captured on the solid support as a function of the test compound concentration.

\section{Protocol Description}

Ki nase assays. For most assays, kinase-tagged $T 7$ phage strains were grown in parallel in 24-well blocks in an E. coli host derived from the BL21 strain. E. coli were grown to log-phase and infected with T7 phage from a frozen stock (multiplicity of infection $=0.4$ ) and incubated with shaking at $32^{\circ} \mathrm{C}$ until lysis $(90-150$ minutes). The lysates were centrifuged $(6,000 \mathrm{xg})$ and filtered $(0.2 \mu \mathrm{m})$ to remove cell debris. The remaining kinases were produced in HEK-293 cells and subsequently tagged with DNA for qPCR detection. Streptavidin-coated magnetic beads were treated with biotinylated small molecule ligands for 30 minutes at room temperature to generate affinity resins for kinase assays. The liganded beads were blocked with excess biotin and washed with blocking buffer (SeaBlock (Pierce), 1\% BSA, $0.05 \%$ Tween $20,1 \mathrm{mM}$ DTT) to remove unbound ligand and to reduce non-specific phage binding. Binding reactions were assembled by combining kinases, liganded affinity beads, and test compounds in $1 x$ binding buffer (20\% SeaBlock, 0.17x PBS, $0.05 \%$ Tween 20, 6 mM DTT). Test compounds were prepared as $40 \mathrm{x}$ stocks in $100 \%$ DMSO and directly diluted into the assay. All reactions were performed in polypropylene 384-well plates in a final volume of $0.02 \mathrm{ml}$. The assay plates were incubated at room temperature with shaking for 1 hour and the affinity beads were washed with wash buffer (1x PBS, $0.05 \%$ Tween 20). The beads were then re-suspended in elution buffer (1x PBS, $0.05 \%$ Tween 20, $0.5 \mu \mathrm{M}$ nonbiotinylated affinity ligand) and incubated at room temperature with shaking for 30 minutes. The kinase concentration in the eluates was measured by qPCR.

\section{Percent Control (XCtrl)}

The compound(s) were screened at the concentration(s) requested, and results for primary screen binding interactions are reported as ' $\%$ Ctrl', where lower numbers indicate stronger hits in the matrix on the following page(s).

\section{XCtrl Calculatio ?}

test compound $=$ compound submitted by $\mathrm{H} 3$ Biomedicine

negative control $=$ DMSO $(100 \%$ Ctrl $)$

positive control $=$ control compound $(0 \% \mathrm{Ctrl})$

\section{Relationship between Binding Constant Distributions (Kds) \&Single Concentration}

\section{Prinary Scree 1 Values}

Based on screening data from thousands of profiled compounds, a proportional relationship between primary screening results and corresponding compound/target affinities may be described. Evident in the correlation graph below is a range of binding constants (Kd values) for the indicated ranges of POC values with tighter binding (higher affinity) interactions associated with lower POC values and weaker binding (lower affinity) associated with higher POC values. This distribution of binding constants is characteristic of single concentration primary screens and underscores the importance of following up observed 'hits' or apparent high affinity interactions with quantitative binding constant determinations.

Data correlation between primary screening $(10 \mu \mathrm{M}$ concentration) and binding constants (Kd values). Binding constants are correlated with primary screening results, where lower POC values are associated with low Kd values (higher affinity interactions).

Data correlation between primary screening (1 $\mu \mathrm{M}$ concentration) and binding constants ( $K d$ values). Binding constants are correlated with primary screening results, where lower POC values are associated with low Kd values (higher affinity interactions).

\section{Refere 7 ces}

$\mathrm{KINOMEscan}^{\mathrm{TM}}$ and BROMOscan ${ }^{\mathrm{TM}}$ use the same assay technology. For a more detailed description of this assay technology, see:

- Fabian, M.A. et al. A small molecule-kinase interaction map for clinical kinase inhibitors. Nat. Biotechnol. 23, 329-336 (2005).

To view kinase interaction maps for 38 well-known kinase inhibitors and a more detailed discussion of selectivity scores, see:

- Karaman, M.W. et al. A quantitative analysis of kinase inhibitor selectivity. Nat. Biotechnol. 26, 127-132 (2008).

Select publications are available at www.discoverx.com.

\begin{tabular}{lrrrrrrrr} 
Kinase & Cmpd 3 & Cmpd 5 & Cmpd 11 & Cmpd 13 & Cmpd 15 & Cmpd 20 & Cmpd 21 & Cmpd 22 \\
ABL1 & 4.7 & 2.7 & 0.1 & 0.05 & 25 & 0.6 & 0 & 12 \\
ABL1 & 2 & 0.25 & 0 & 0 & 17 & 0.2 & 0 & 2.9 \\
AURKA & 1.4 & 1 & 5 & 14 & 5.3 & 10 & 1.5 & 26 \\
AXL & 4.1 & 0.15 & 7.8 & 0 & 26 & 0.05 & 1.3 & 11 \\
CSF1R & 62 & 38 & 28 & 1.4 & 92 & 65 & 60 & 79 \\
DDR2 & 28 & 1.7 & 0 & 0 & 70 & 5.4 & 0.6 & 45 \\
EGFR & 100 & 98 & 88 & 55 & 91 & 83 & 49 & 100 \\
\hline FGFR1 & 2.6 & 2.8 & 3 & 1.6 & 29 & 8.9 & 17 & 14
\end{tabular}




\begin{tabular}{lrrrrrrrr|} 
FGFR2 & 11 & 2.1 & 8.9 & 6.4 & 55 & 39 & 48 & 39 \\
FGFR3 & 3.8 & 0.95 & 1.5 & 0.35 & 33 & 23 & 40 & 25 \\
FGFR4 & 32 & 25 & 75 & 14 & 100 & 99 & 100 & 100 \\
\hline FGR & 1.7 & 0.8 & 0.35 & 0.3 & 19 & 1.5 & 1.1 & 29 \\
FLT3 & 0 & 0 & 0.35 & 0 & 0 & 0 & 0 & 1.4 \\
KIT & 8.3 & 3.2 & 1.3 & 0.15 & 32 & 21 & 6.1 & 52 \\
LIMK1 & 34 & 57 & 36 & 16 & 100 & 100 & 80 & 100 \\
MERTK & 71 & 6.9 & 54 & 0.35 & 90 & 1.4 & 37 & 13 \\
MET & 100 & 60 & 100 & 22 & 53 & 49 & 100 & 26 \\
PDGFRA & 16 & 16 & 6.8 & 16 & 35 & 31 & 8.3 & 41 \\
PDGFRB & 13 & 4.7 & 0.95 & 0.25 & 41 & 12 & 2.8 & 54 \\
RET & 0 & 0 & 0.6 & 0.25 & 0.4 & 0 & 0.2 & 14 \\
SRC & 0 & 0.15 & 0.25 & 0 & 4.1 & 0.1 & 0.1 & 2.9 \\
TNK1 & 3.6 & 1.4 & 1.8 & 0.7 & 15 & 18 & 12 & 21 \\
KDR & 1.8 & 1.1 & 2.4 & 8.2 & 9.3 & 5.4 & 3.1 & 40 \\
YES1 & 7.1 & 0.95 & 0.5 & 0.15 & 58 & 0.35 & 0.3 & 9.6
\end{tabular}

\section{Institutional Animal Care and Use Committee Statement:}

All mouse studies were conducted in compliance with all applicable regulations and guidelines of the Institutional Animal Care and Use Committee (IACUC) from the National Institutes of Health (NIH). Mice were maintained under pathogen-free conditions, and food and water was provided ad libitum. 